\title{
Stabilisierung und Kontrolle komplexer Dynamik durch mehrfach zeitverzögerte Rückkopplung
}

\section{Dissertation}

zur Erlangung des Doktorgrades

der Mathematisch-Naturwissenschaftlichen Fakultäten

der Georg-August-Universität zu Göttingen

vorgelegt von

\section{Alexander Ahlborn}

aus Göttingen

Göttingen 2007 
Referent

Korreferent
Prof. Dr. Ulrich Parlitz

Prof. Dr. Eberhard Bodenschatz

Tag der mündlichen Prüfung: 


\section{Inhaltsverzeichnis}

1 Einleitung $\quad 7$

2 Regelung von chaotischen Systemen $\quad 11$

2.1 Konventionelle Regelungsmethoden . . . . . . . . . . . . . . . . . . . 11

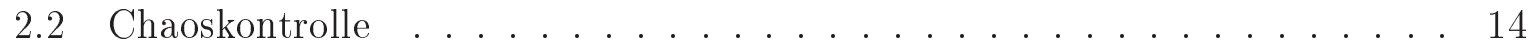

2.3 Multiple Delay Feedback Control _ . . . . . . . . . . . . . . . . 16

2.4 Lineare Stabilitätsanalyse . . . . . . . . . . . . . . . . . 17

2.5 Beispiel - der Colpitts-Oszillator . . . . . . . . . . . . . . . . 19

2.6 Vergleich zu anderen Regelungsmethoden . . . . . . . . . . . . . . . . 24

2.7 Wirkungsweise im Fourierraum . . . . . . . . . . . . . . . . 26

2.8 Parameterwahl . . . . . . . . . . . . . . . . . . . . . . . . . . . 29

2.8.1 Rückkopplung ohne Totzeit . . . . . . . . . . . . . . . . . . 29

2.8.2 Rückkopplung mit Totzeit . . . . . . . . . . . . . . . . . . 38

2.9 Koexistierende Fixpunkte: Anfangswertproblem . . . . . . . . . . . . 43

2.10 Realisierungen von Zeitverzögerungen . . . . . . . . . . . . . . . . 45

2.11 Weitere Details . . . . . . . . . . . . . . . 47

3 Von MDFC zu NFF $\quad 49$

3.1 Übergang vom Zeit- in den Frequenzbereich . . . . . . . . . . . . . . . . . 49 
4 Kontrollierbarkeit von Fixpunkten $\quad 55$

4.1 Allgemeines . . . . . . . . . . . . . . . . . . 55

4.2 Multiple Delay Feedback Control . . . . . . . . . . . . . . . . . . . 55

4.3 Notch Filter Feedback und Hochpassfilter . . . . . . . . . . . . . . . . 58

4.4 Periodische Orbits . . . . . . . . . . . . . . . . . 62

5 Kontrolle chaotischer Laserdynamik $\quad 63$

5.1 Holografische Displays . . . . . . . . . . . . . . . . 63

5.2 Frequenzverdopplung . . . . . . . . . . . . . . . . 64

5.3 Lasermodell . . . . . . . . . . . . . . . . . . . . . 66

5.4 Der experimentelle Aufbau . . . . . . . . . . . . . . . . . . . . 70

5.5 Laser-Stabilisierung . . . . . . . . . . . . . . . . . . . . . . 73

5.6 Regelung des Lasers in der Praxis . . . . . . . . . . . . . . . . . . . . 75

5.7 Regelung des Lasers in der Simulation . . . . . . . . . . . 78

5.8 Phasensynchronisation . . . . . . . . . . . . . . . . . . . . . 83

6 Räumlich ausgedehnte Systeme $\quad 89$

6.1 Allgemeines . . . . . . . . . . . . . . . . . . . . . 89

6.2 Die Ginzburg-Landau-Gleichung . . . . . . . . . . . . . . . . . . . . . 93

6.3 Stabilisierung ebener Wellen . . . . . . . . . . . . . . . . . . . . . 93

6.4 Inhomogene Rückkopplung . . . . . . . . . . . . . . . . . 95

6.5 Lokale Rückkopplung . . . . . . . . . . . . . . . . . . 97

6.6 Synchronisation . . . . . . . . . . . . . . . 105

7 Zusammenfassung und Ausblick $\quad 111$

7.1 Zusammenfassung . . . . . . . . . . . . . . . . 111

7.2 Ausblick . . . . . . . . . . . . . . . . . . . 113

A Chaos-Kontrollmethoden ohne Zeitverzögerung 115

A.1 OGY-Kontrolle . . . . . . . . . . . . . . . . . . 115

A.2 OPF-Kontrolle . . . . . . . . . . . . . . . . . 118 
B Kontrollierbarkeitskriterium im allgemeinsten Fall

C Räumlich ausgedehnte Systeme 125

C.1 Ginzburg-Landau-Gleichung (Stabilitätsanalyse) . . . . . . . . . . . 125

C.2 Ginzburg-Landau-Gleichung (Fixpunkt) . . . . . . . . . . . . . . 125

C.3 Fitzhugh-Nagumo-System . . . . . . . . . . . . . . . 127 


\section{Kapitel 1}

\section{Einleitung}

Die Welt, in der wir leben, ist nichtlinear. Beispiele für diesen Sachverhalt gibt es zahlreich, überall, jeden Tag. Viele dieser komplexen Vorgänge lassen sich durch Formeln beschreiben und dennoch können für diese an sich rein deterministischen Systeme keine Langzeitvorhersagen vorgenommen werden. Vielmehr ist eine sensitive Abhängigkeit von den Anfangswerten festzustellen, die zu extrem unterschiedlichen Dynamikverläufen führen kann. Solch chaotisches Verhalten resultiert in vielen Fällen aus nichtlinearen Kopplungen der Systemvariablen untereinander.

Eine besondere Klasse nichtlinearer Systeme unterliegt zudem zeitverzögerter Dynamik, deren Beschreibung üblicherweise durch sogenannte Delay Differential Equations (DDEs) vorgenommen werden muss. Beispiele für diesen Zusammenhang findet man in vielfältiger Art in der Biologie, Medizin und Technik [32, 38, 52, 87, 90, 132, 135, 154, 156]. Die angesprochene Zeitverzögerung ist das Ergebnis einer endlichen Signalausbreitungsgeschwindigkeit einer oder mehrerer Systemvariablen. Oft ist mit dem Vorhandensein zeitverzögerter Größen periodische oder u. U. hochdimensionale chaotische Dynamik verknüpft $[10,44,65,139,153]$. Allerdings sind die erwähnten periodischen oder chaotischen Oszillationen in Anwendungen häufig unerwünscht und stellen sich als widrige Begleiterscheinungen eines gewünschten Effektes, der lediglich durch den Einsatz nichtlinearer Systeme erreicht werden kann, heraus. Die dafür benötigten Systeme können ohne den Verlust ihrer gewünschten Eigenschaften nicht mehr wie bisher üblich auf einen linearen und damit vorhersagbaren Arbeitsbereich eingeschränkt werden. In solchen Fällen muss dann eine geeignete Regelung implementiert werden, um die Nichtlinearität nicht zu zerstören, sondern um die benötigten (chaotischen) Systeme zu stabilisieren und für zukünftige Anwendungen handhabbar zu machen. Dabei besitzt die Stabilisierung von Fixpunkten, auf die in dieser Arbeit hauptsächlich eingegangen wird, eine besondere Bedeutung. Erreicht wird dieses Ziel durch eine geeignete Veränderung eines zugänglichen Systemparameters oder einer Systemvariablen. Aufgrund der internen Kopplung der Systemvariablen reicht bei einem nichtlinearen System die Beeinflussung einer einzigen Variablen oder eines Parameters aus, um alle Variablen zu verändern. Dieses Vorgehen führt bei falschen 
Regelparametern zu einer komplizierteren Dynamik bzw. bei geeigneter Parameterwahl zu der gewünschten einfachen Zieldynamik (in diesem Fall einem Fixpunkt). Das Problem besteht allerdings darin, dass die Dynamik des zu regelnden Systems wegen der sensitiven Abhängigkeit von den Anfangswerten u. U. nur sehr schwer vorhersagbar ist, womit sich natürlich auch eine Vorausberechnung des für die Zieldynamik nötigen Regelsignals u. U. kompliziert gestaltet. Besondere Schwierigkeiten bereiten in diesem Zusammenhang schnelle und/oder hochdimensionale (raum-) zeitlich chaotische Systeme.

Um deren komplexe Dynamik zu zähmen, wurden in den letzten 15 Jahren große Anstrengungen unternommen. Zum Einsatz kam dabei neben Steuerungen [62] und konventionellen Regelungen (z. B. PID-Regelung [150]) eine Vielzahl alternativer Kontrollmethoden, die z. B. auf lokalen Approximationen einer geeignet gewählten Poincaré-Ebene beruhen [136]. Daneben wurden Methoden eingesetzt, die auf der Verwendung einer Zeitverzögerung basieren [117,141], was in Anbetracht der vorherigen Ausführungen zunächst kontraintuitiv wirkt, aber dennoch erfolgreich ist. Unglücklicherweise sind die genannten Regelungen nicht universell für alle dynamischen Systeme anwendbar, sondern unterliegen ernsthaften Beschränkungen.

Zur Umgehung dieser Beschränkungen habe ich die beiden neuartigen Regelungsmethoden Multiple Delay Feedback Control (MDFC) [4-6,9] und Notch Filter Feedback (NFF) [8] vorgeschlagen. Während bei MDFC die Wirkung gewichteter Differenzen mehrerer unterschiedlich lang verzögerter Signale und deren unverzögerter Größen ausgenutzt wird, basiert NFF auf der Verwendung von mehreren Kerbfiltern unterschiedlicher Resonanzfrequenz. Im Zuge dieser Arbeit werden sich MDFC und NFF allen anderen bisherigen Regelungsmethoden wie (E)TDAS, NTDAS, P(roportional)- und PD-Regelung als überlegen herausstellen. Das gilt hinsichtlich der Güte der Fixpunktstabilisierung als auch in Bezug auf die Einsatzfähigkeit und Effizienz. Zudem ist aufgrund einer starken Erweiterung der Stabilitätsgebiete im Vergleich zu alternativen Regelungsmethoden eine erfolgreiche Stabilisierung von Fixpunkten in Gegenwart langer Verzögerungszeiten möglich. Erreicht werden diese Vorteile für MDFC durch die individuelle Gewichtung der einzelnen verzögerten und unverzögerten Terme. Dadurch kann das Regelsignal besser an das zu regelnde dynamische System angepasst werden, als es bei bisherigen Regelungen mit vergleichbarem Aufwand der Fall ist. Diese Sachverhalte werden im anschließenden zweiten Kapitel anhand von Simulationen untermauert. Da die analytische Untersuchung von Delay Differential Equations schwierig bzw. für mehrere unabhängige Verzögerungszeiten unmöglich ist, muss sich ein Teil der Untersuchungen allerdings Spezialfällen widmen, um analytisch exakte Lösungen zu liefern.

Vergleichbare Ergebnisse wie mit MDFC im Zeitbereich können durch das sogenannte Notch Filter Feedback (NFF) [8] im Fourierraum erzielt werden. Dabei wird die MDFCÜbertragungsfunktion lokal durch parallel angeordnete und individuell gewichtete Kerbfilter approximiert. Im Rahmen des dritten Kapitels wird sich die Effizienz dieser Methode genau wie für MDFC anhand von Stabilitätsanalysen in Simulationen herausstellen. 
Ähnlich wie bei periodischen Orbits unterliegen die bekannten Zeitverzögerungsregelungen (E)TDAS auch allgemeinen Beschränkungen bei der Fixpunktstabilisierung. So lassen sich im Gegensatz zu (E)TDAS instabile Sattelpunkte mit MDFC und NFF kontrollieren bzw. stabilisieren. Diese vorteilhafte Eigenschaft der beiden zuletzt genannten Regelungen wird im vierten Kapitel mathematisch bewiesen, durch Simulationen veranschaulicht und zeigt die Überlegenheit von NFF und MDFC gegenüber den anderen bekannten Regelungsmethoden. Dieser Sachverhalt spiegelt sich auch bei der erfolgreichen experimentellen Stabilisierung eines kompakten, intern frequenzverdoppelten Nd:YAG Lasers auf eine konstante Ausgangsintensität mit Hilfe von MDFC oder NFF wider. Dieser Lasertyp wird häufig zur Erzeugung von grünem oder blauem Laserlicht verwendet. Unglücklicherweise treten dabei (chaotische) Intensitätsschwankungen auf, wenn z. B. der Pumpstrom des Lasers einen bestimmten kritischen Wert überschreitet. Dieses seit 20 Jahren bestehende green problem [64,65] zeichnet sich durch eine äußerst schwer zu zähmende Dynamik aus, die bisher den Einsatz derartiger frequenzverdoppelter Laser in Anwendungen wie holografischen Displays oder der Datenübertragung und Codierung behindert. Sowohl mit MDFC als auch mit NFF konnte das green problem sehr stark reduziert werden. Die entsprechenden Resultate, die auch durch ausgedehnte Simulationen an einem geeigneten, detaillierten Lasermodell bestätigt wurden, sind dem fünften Kapitel zu entnehmen. In diesem Zusammenhang wird auch die technisch relevante Möglichkeit einer langsamen periodischen Modulation des stabilisierten Lasers zum Zweck der Informationsübertragung untersucht, was z. B. für die Realisierung von farbigen holografischen Displays entscheidend ist. Diese Modulation stellt zusätzlich zu der aktivierten Regelung eine Steuerung des Lasers dar. Wird nun die Regelung bei aktivierter Steuerung abgeschaltet, tritt bei geeigneter Parameterwahl (chaotische) Phasensynchronisation auf, die im Zuge dieser Arbeit experimentell bei dem untersuchten Lasersystem nachgewiesen werden konnte. Die entsprechenden Ergebnisse sind ebenfalls dem fünften Kapitel zu entnehmen.

Der Übergang zu räumlich ausgedehnten Systemen wird schließlich im sechsten Kapitel vorgenommen. Im Vordergrund steht hierbei die Kontrolle der komplexen zweidimensionalen Ginzburg-Landau-Gleichung (GLE), die je nach Parameterwahl Spiralchaos oder turbulente Dynamik offenbart. Dabei liegt neben der Unterdrückung chaotischer Oszillationen ein Fokus auf der Umwandlung von Spiralwellen oder turbulenter Dynamik in laufende ebene Wellen, was lediglich mit global oder lokal angewendetem MDFC für alle GLE-Parameter möglich ist. Die Geschwindigkeit dieser ebenen Wellen kann wiederum durch die MDFC-Parameter verändert werden, die zudem bei lokaler Anwendung noch die Möglichkeit weiterer Manipulationen wie sektorgerichteter oder gescherter ebener Wellen oder Spiralfallen bieten. Die Betrachtung der für die lokale Anwendung des Regelsignals erforderlichen Kontrollzellen führt für Spiralfallen wiederum auf den Vorgang der Synchronisation, der im sechsten Kapitel untersucht wird.

Als Abschluss umfasst das siebte Kapitel eine kurze Zusammenfassung der bis dahin vorgestellten Ergebnisse sowie einen kleinen Ausblick auf daraus resultierende Fragestellungen. Zum Abrunden dieser Ausführungen enthält der Anhang zudem weitere Informationen zu 
Chaos-Kontrollmethoden, allgemeinen Kontrollierbarkeitskriterien von Fixpunkten mit Hilfe von MDFC sowie weitere Ausführungen zur Kontrolle von raum-zeitlichem Chaos. Unter anderem kann dabei das bekannte Fitzhugh-Nagumo-System mit Hilfe von MDFC und NFF auf laufende Wellen stabilisiert werden, was wiederum Relevanz für eine mögliche Vermeidung von Herz-Rhythmus-Störungen im menschlichen Herzgewebe haben kann. 


\section{Kapitel 2}

\section{Regelung von chaotischen Systemen}

\subsection{Konventionelle Regelungsmethoden}

Die heutige Welt ist ohne den Einsatz von Regelungen und Steuerungen nicht mehr vorstellbar. Man denke hierbei an übliche Heizungen (Zweipunktregelung ${ }^{1}$ ), Niveauregelungen (Dreipunktregler) oder auch die Stabilisierung von Motordrehzahlen, Schrittmotorsteuerung etc.. Allgemein soll mit Hilfe einer Regelung oder Steuerung eine physikalische Größe konstant gehalten oder in einer bestimmten Art und Weise verändert werden. Dabei bedient sich die Steuerung im Gegensatz zu einer Regelung einer einseitigen, d. h. unidirektionalen Signalübertragung, ohne ständig den Systemzustand in Form einer Messgröße zu detektieren. Eine Regelung bzw. Regelstrecke hingegen besteht aus einer Messeinrichtung, einer geeignet gewählten Regelgröße sowie dem eigentlichen Regler, der das notwendige Regelsignal generiert und entsprechende Abweichungen in Form einer Gegenkopplung korrigiert. Die wohl am häufigsten verbreitete Regelungstopologie ist der PID-Regler [86, 150]. Dabei wird der momentane Systemfehler $e(t)=r(t)-y(t)$ zwischen der Sollgröße $r(t)$ und dem Messsignal $y(t)=\mathbf{g}(\mathbf{x}(t))$ des Systemzustandes $\mathbf{x}$ festgestellt und mittels eines Proportionalanteils (P), eines Integralanteils (I) sowie eines Differenzierungsanteils (D) wieder dem dynamischen System zugefügt. Insgesamt ergibt sich das Regelsignal

$$
u(t)=k_{p} e(t)+k_{i} \int_{-\infty}^{t} e(t) \mathrm{d} t+k_{d} \frac{\mathrm{d} e(t)}{\mathrm{d} t}
$$

\footnotetext{
${ }^{1}$ Konventionelle Regler können in stetige und unstetige Regler eingeteilt werden. Stetige Regelungen sind beispielsweise P-, PD-, PI oder PID-Kontroller, die jeden beliebigen Wert des Kontrollsignals annehmen können, wohingegen Zwei-, Drei oder Mehrpunktregler unstetige Vertreter von Regelungen sind. So besitzt der Zweipunktregler nur zwei Zustände des Regelsignals, was einem Schalter mit den Stellungen AN und AUS entspricht. Sind entsprechend mehr unterschiedliche Schalterstellungen möglich, wird von einem Dreipunkt- oder Mehrpunktregler gesprochen. Eingesetzt wird diese Regeltopologie in Systemen, in denen große Zeitkonstanten der Regelstrecke auftreten [144].
} 
wobei die Verstärkungsfaktoren $k_{p}, k_{i}$ und $k_{d}$ so zu wählen sind, dass die gesamte Regelstrecke nicht selbständig zu schwingen anfängt. Zu diesem Zweck wurden verschiedene Strategien entwickelt [85], die im Zuge der optimalen Kontrolle zudem versuchen, die Regelparameter entsprechend einer zu minimierenden system- und dynamikbezogenen Kostenfunktion anzupassen. Häufig wird dazu noch eine adaptive Komponente benötigt, die die Regelparameter in Gegenwart von Systeminstationarität entsprechend nachführt und den unterschiedlichen Dynamikbereichen anpasst.

Neben dem PID-Regler gibt es zudem Regelungen, die auf sogenannten Zustandsrückführungen basieren. Hierbei handelt es sich um Mehrgrößenregelungen, bei denen der Zustandsvektor $\mathbf{x}(t)$ mittels geeigneter Parameter wieder auf das System zurückgekoppelt wird. Die verbreitetste Ausführungsform hierfür ist das in den 1950er Jahren entwickelte Pole Placement [136], für das im Gegensatz zum PID-Regler vor dem Generieren des Regelsignals eine Systemanalyse vorgenommen werden muss. Um die für die beabsichtigte Zieldynamik notwendigen Kontrollparameter zu ermitteln, wird an dieser Stelle das zeitdiskrete System

$$
\dot{\mathbf{x}}_{n}=\mathbf{f}\left(\mathbf{x}_{n}, p_{n}\right), \quad \mathbf{x}_{n} \in \mathbb{R}^{N}
$$

mit der glatten Vektorfunktion $\mathbf{f}\left(\mathbf{x}_{n}, p_{n}\right)$ sowie dem extern zugänglichen Systemparameter $p_{n}$ betrachtet. Da nur kleine Störungen zur Änderung der Systemdynamik zum Einsatz kommen sollen (d.h. $\left|p_{n}-p_{0}\right| \leq \delta$ ), wird $p_{n}-p_{0}=0$ gesetzt, wenn die Systemdynamik noch weit von der Zieldynamik entfernt ist. $p_{0}$ bezeichnet den zu variierenden Systemparameter bei Erreichen der beabsichtigten Zieldynamik, z. B. am Fixpunkt $\mathbf{x}_{0}\left(p_{0}\right)$. In einer kleinen Umgebung um diesen Fixpunkt kann das Vektorfeld linearisiert werden, was unter Verwendung der $N \times N$-Jacobi-Matrix $D_{x} \mathbf{f}$ und dem $N$-dimensionalen Spaltenvektor $D_{p} \mathbf{f}$ auf

$$
\mathbf{x}_{n+1}-\mathbf{x}_{0}\left(p_{0}\right)=\left.D_{x} \mathbf{f}\left(\mathbf{x}_{n}, p\right)\right|_{\mathbf{x}_{0}\left(p_{0}\right)}\left(\mathbf{x}_{n}-\mathbf{x}_{0}\left(p_{0}\right)\right)+\left.D_{p} \mathbf{f}\left(\mathbf{x}_{n}, p\right)\right|_{p_{0}}\left(p_{n}-p_{0}\right)
$$

führt. Die zeitabhängige Störung berechnet sich unter diesem Gesichtspunkt zu

$$
p_{n}-p_{0}=-\mathbf{k}^{t r} \cdot\left(\mathbf{x}_{n}-\mathbf{x}_{0}\left(p_{0}\right)\right), \quad \mathbf{k}^{t r} \in 1 \times N
$$

mit dem zu ermittelnden (transponierten) Verstärkungsvektor $\mathbf{k}^{\text {tr }}$. Einsetzen von Gl. (2.4) in Gl. (2.3) resultiert in

$$
\mathbf{x}_{n+1}-\mathbf{x}_{0}\left(p_{0}\right)=\left(\left.D_{x} \mathbf{f}\left(\mathbf{x}_{n}, p_{n}\right)\right|_{\mathbf{x}_{0}\left(p_{0}\right)}-\left.D_{p} \mathbf{f}\left(\mathbf{x}_{n}, p\right)\right|_{p_{0}} \cdot \mathbf{k}^{t r}\right)\left(\mathbf{x}_{n}-\mathbf{x}_{0}\left(p_{0}\right)\right) .
$$

Ziel ist es nun, durch Anpassung von $\mathbf{k}^{t r}$ an das Kontrollproblem den betrachteten Fixpunkt zu stabilisieren, d.h. alle Eigenwerte von $A-B \mathbf{k}^{t r}$ mit $A=\left.D_{x} \mathbf{f}\left(\mathbf{x}_{n}, p\right)\right|_{\mathbf{x}_{0}\left(p_{0}\right)}$ und $B=\left.D_{p} \mathbf{f}\left(\mathbf{x}_{n}, p\right)\right|_{p_{0}}$ vom Betrag kleiner als eins zu gestalten. Unter der Annahme, dass die Matrix

$$
C=\left(B|A B| A^{2} B|\cdots| A^{N-1} B\right)
$$


vollen Rang hat, lässt sich die Lösung dieses Kontrollproblems zu

$$
\mathbf{k}^{t r}=\left(\alpha_{N}-a_{N}, \ldots, \alpha_{1}-a_{1}\right) E^{-1}, \quad E=C W
$$

mit

$$
W=\left(\begin{array}{ccccc}
a_{N-1} & a_{N-2} & \cdots & a_{1} & 1 \\
a_{N-2} & a_{N-3} & \cdots & 1 & 0 \\
\vdots & \vdots & \vdots & \vdots & \vdots \\
a_{1} & 1 & \cdots & 0 & 0 \\
1 & 0 & \cdots & 0 & 0
\end{array}\right)
$$

angeben [136]. $a_{1}, \ldots, a_{N}$ bezeichnet in diesem Zusammenhang die Koeffizienten des charakteristischen Polynoms von $A$, und $\alpha_{1} \ldots \alpha_{N}$ in analoger Weise die entsprechenden Koeffizienten des gewünschten charakteristischen Polynoms von $\left(A-B \mathbf{k}^{t r}\right)$.

Mit dem Beginn der Chaosforschung wurden die bisher genannten und für die Beeinflussung von linearen Systemen entwickelten Regelungsstrategien eingesetzt, um auch chaotische Systeme ${ }^{2}$ für praktische Anwendungen handhabbar zu machen. Im Zuge der Chaoskontrolle wird zu diesem Zweck im besten Fall nur eine einzige Systemobservable detektiert, um das betrachtete System hinsichtlich der gewünschten Zieldynamik zu beeinflussen. ${ }^{3}$ Die Grundvoraussetzung der Chaoskontrolle ist die Tatsache, dass es eine unendliche Zahl von periodischen Orbits ${ }^{4}$ und eine oft begrenzte Anzahl an (instabilen) Fixpunkten gibt, die in einem chaotischen Attraktor ${ }^{5}$ eingebettet sind [14]. Wegen der Ergodizität erreicht die Dynamik in endlicher Zeit die Nachbarschaft jeden dieser Orbits oder Fixpunkte, wodurch unter Ausnutzung der sensitiven Abhängigkeit von den Anfangsbedingungen kleine Störungen dazu verwendet werden können, das Systemverhalten in bestimmte Bahnen zu lenken und damit auftretendes Chaos durch kleine Parameteränderungen zu regeln bzw. zu eliminieren [106, 136]. Zu Beginn der Chaoskontrolle stand dabei die Stabilisierung periodischer Orbits im Vordergrund, wofür die bisher genannten

\footnotetext{
${ }^{2}$ Nichtlineare bzw. chaotische Systeme zeigen in ihrer Dynamik eine empfindliche Abhängigkeit von den Anfangsbedingungen, so dass ihr Verhalten nicht langfristig vorhergesagt werden kann. Da diese Systeme physikalischen Gesetzen unterliegen, die Dynamik aber gleichzeitig scheinbar irregulär ist, bezeichnet man sie als deterministisches Chaos [14].

${ }^{3}$ Durch die Kopplung der Systemvariablem bei nichtlinearen bzw. chaotischen Systemen reicht die Veränderung einer Variablen aus, um alle Systemvariablen zu beeinflussen [136].

${ }^{4}$ Die Dynamik innerhalb eines chaotischen bzw. nichtlinearen Systems kann verschiedene Formen annehmen. Die Palette der Variationen reicht von Fixpunkten über periodisches Verhalten (Orbits) und quasiperiodischer Dynamik bis hin zu Chaos. An den Übergängen zwischen diesen verschiedenen Systemverhaltensweisen finden Bifurkationen (qualitative Änderungen der Dynamik) statt, die durch Parameteränderungen bedingt sind [14].

${ }^{5}$ Ein Attraktor ist eine unter der Zeitentwicklung eines dynamischen Systems invariante (d.h. sich zeitlich nicht ändernde) Untermenge eines Phasenraums, die durch die Dynamik dieses Systems nicht mehr verlassen wird. Die Menge aller Punkte des Phasenraums, die unter der Dynamik demselben Attraktor zustreben, werden als Bassins dieses Attraktors bezeichnet [14].
} 
Regelungsmethoden allerdings nur bedingt geeignet sind. Folglich wurde in den vergangenen 15 Jahren eine Vielzahl alternativer Methoden zur Chaoskontrolle vorgeschlagen und theoretisch wie auch experimentell untersucht [136].

\subsection{Chaoskontrolle}

Einer der ersten Ansätze chaotisches Verhalten zu unterdrücken, wurde von Hübler und Lüscher 1989 in Form von Steuerungsmethoden vorgeschlagen [62]. Die Anwendung von Steuerungsmethoden ist oft einfacher als die einer Regelung, da die Wirkungsweise unidirektional ist, was die Schwingneigung im Gesamtsystem minimiert. Allerdings wird sich dieser Vorteil oft durch eine höhere benötigte Verstärkung im Vergleich zu Regelungsmethoden erkauft. Nachteilig ist zudem, dass die Kontrollparameter zum Erreichen der gewünschten Zieldynamik (z. B. eines periodischen Orbits) häufig durch Fehlschlagmethoden bestimmt werden müssen.

Alternativ hierzu veröffentlichten Ott, Grebogi und Yorke (OGY) 1991 eine Regelungsmethode [106], die im wesentlichen auf einer lokalen Anwendung des Pole-Placement beruht und eine Vielzahl von Veröffentlichungen bezüglich Chaoskontrolle und alternativer Regelungsideen nach sich zog [136]. Im Detail bedienen sich OGY lokaler Approximationen einer geeigneten Poincaré-Abbildung ${ }^{6}$ im Einbettungsraum ${ }^{7}$, d. h. die Systemgleichungen müssen zu diesem Zweck entweder bereits bekannt sein oder mit Hilfe einer Zeitreihenanalyse (lokal) approximiert werden. Ein Beispiel für die Stabilisierung eines Fixpunktes mit der OGY-Methode befindet sich im Anhang ab Seite 115. Für eine experimentelle Anwendung wird hierfür zunächst eine A/D-Wandlung, anschließend numerische Berechnungen und am Ende wiederum eine D/A-Wandlung benötigt. Dieser Umstand macht es sehr schwierig, die OGY-Kontrolle experimentell auf schnelle dynamische Systeme anzuwenden.

Alternativen zur OGY-Kontrolle, die auch mit analoger Elektronik und ohne Computereinsatz implementiert werden können, sind z. B. Occasional Proportional Feedback (OPF)

\footnotetext{
${ }^{6}$ Die Poincaré-Ebene ist eine geeignet gewählte Ebene im Phasenraum eines zeitkontinuierlichen dynamischen Systems, die von den Trajektorien transversal geschnitten wird. Durch die Betrachtung einer Ponicaré-Ebene wird zum einen die Dimension des Systems um eins reduziert und zum anderen der Übergang von einem kontinuierlichen System zu einer diskreten, iterierten Abbildung vorgenommen. Die Abbildung, die einen (Durchstoß)Punkt der Ebene auf den nächsten abbildet, wird als Poincaré-Abbildung bezeichnet [14].

${ }^{7}$ In den meisten experimentellen Fällen sind die Zustandsgleichungen a priori unbekannt. Um einen Attraktor aus einer in konstanten Abständen $\Delta t$ gemessenen Zeitreihe rekonstruieren zu können, wird die Zeitreihe $S_{n}=\mathbf{g}(\mathbf{x}(n \Delta t))$ entsprechend eingebettet. $\mathbf{x}$ bezeichnet den Zustand des untersuchten Systems, das in skalarer Form durch die Beobachtungsfunktion $\mathbf{g}$ vorliegt. Die Einbettung wird dann üblicherweise durch die Bildung von Zeitverzögerungsvektoren der Form $\zeta_{n}=\left(S_{n}, S_{n+\tau}, \ldots, S_{n+(d-1) \tau}\right)$ mit der Verzögerungszeit $\tau$ vorgenommen. Auf diese Weise besteht bei entsprechend großer Wahl der ganzzahligen Einbettungsdimension $d$ ein Diffeomorphismus zwischen dem eingebetteten Attraktor und dem wirklich zugrunde liegenden [14].
} 
[63,98] (Details im Anhang ab Seite 118) oder Methoden, die auf Zeitverzögerung basieren. Diese zuletzt genannten Methoden werden auch den Hauptschwerpunkt dieser Arbeit bilden, wobei die wohl bekannteste Ausführungsform dieser Art der Rückkopplung die sogenannte Pyragas-Kontrolle Time Delay Auto Synchronization (TDAS) [70, 102, 117] ist. Ähnlich wie die OGY-Kontrolle - aber wesentlich einfacher im Experiment zu implementieren - wurde TDAS zunächst zur Stabilisierung von instabilen periodischen Orbits eingesetzt $[26,34,49,63,70,98,106,117,136,140,141,145]$. Dabei ist das Regelsignal die verstärkte Differenz einer geeigneten Messfunktion (in den meisten Fällen eine skalare Größe), die zu den Zeiten $t$ und $t-\tau$ gemessen wird, was bei Betrachtung des dynamischen Systems

$$
\dot{\mathbf{x}}=\mathbf{f}(\mathbf{x}, \mathbf{u})
$$

zu dem TDAS-Signal

$$
\mathbf{u}(t)=k[\mathbf{g}(\mathbf{x}(t-\tau))-\mathbf{g}(\mathbf{x}(t))]
$$

führt. u bezeichnet das Rückkopplungssignal, $k$ ist ein angepasster Verstärkungsfaktor und $\mathbf{g}$ definiert die verwendete Messfunktion, um den Zustand $\mathbf{x}$ des betrachteten dynamischen Systems zu detektieren. Die Verzögerungszeit $\tau$ wird üblicherweise entsprechend der Periode des zu stabilisierenden (instabilen) periodischen Orbits (UPO) gewählt. In diesem Fall verschwindet das Regelsignal für den betrachteten UPO, wodurch dieser nicht verzerrt wird, sondern lediglich seine Stabilitätseigenschaften ändert. Folglich spricht man von nichtinvasiver Kontrolle.

Eine Verallgemeinerung von TDAS ist durch Extended Time Delay Auto Synchronization (ETDAS) aus dem Jahre 1994 gegeben [34, 49,102,141,145]. Im Gegensatz zu TDAS berücksichtigt dieses von Gauthier et al. vorgeschlagene Schema auch noch die verwendete Observable zu ganzzahligen Vielfachen der fundamentalen Verzögerungszeit $\tau$ in Form einer geometrischen Reihe. Unter Berücksichtigung derselben Abkürzungen wie in (2.10), ist ETDAS durch

$$
\begin{aligned}
\mathbf{u}(t) & =k\left[(1-R) \sum_{j=1}^{\infty} R^{j-1} \mathbf{g}(\mathbf{x}(t-j \tau))-\mathbf{g}(\mathbf{x}(t))\right] \\
& =k[\mathbf{g}(\mathbf{x}(t-\tau))-\mathbf{g}(\mathbf{x}(t))]+R \mathbf{u}(t-\tau)
\end{aligned}
$$

gegeben, wobei $R \in$ ] -1,1 [ ein Gewichtungsfaktor für die Terme der geometrischen Reihe ist. In Experimenten kann ETDAS ebenfalls mit einer einzigen Verzögerungskette implementiert werden, indem einfach die entsprechenden Signale mehrfach durch die Kette geleitet und dabei jeweils mit dem Faktor $R$ multipliziert werden. Untersuchungen haben gezeigt, dass ETDAS höhere Stabilitätswerte und ausgedehntere Stabilitätsgebiete für die Stabilisierung von periodischen Orbits liefert als TDAS. Für Fixpunktstabilisierung schlagen Chang et al. die Betrachtung des Grenzfalls $R \rightarrow 1, \tau \rightarrow 0$ vor [34]. Unter diesem Gesichtspunkt kann Gl. (2.12) in eine Taylor-Reihe entwickelt werden, was letztlich zu

$$
\dot{\mathbf{u}}(t)=-\omega_{0} \mathbf{u}(t)-k \dot{\mathbf{g}}(\mathbf{x}(t))
$$


führt. Dabei handelt es sich um einen Hochpassfilter mit der Grenzfrequenz $\omega_{0}=(1-R) / \tau$, der Verstärkung $k$ sowie einem einfachen Pol bei $\omega=i \omega_{0}$ und einer Nullstelle bei $\omega=0$.

Neben (E)TDAS gibt es noch das wesentlich unbekanntere NTDAS ( $N$ Time Delay Auto Synchronization) [140], bei dessen Anwendung der Mittelwert einer endlichen Anzahl von Verzögerungstermen benutzt wird, um das Regelsignal zu bilden. Aber genau wie bei ETDAS werden auch hierbei nur ganzzahlige Vielfache einer einzigen fundamentalen Verzögerungszeit $\tau$ verwendet, d. h. das NTDAS- Kontrollsignal lautet

$$
\mathbf{u}(t)=k\left[\frac{1}{N} \sum_{j=1}^{N} \mathbf{g}(\mathbf{x}(t-j \tau))-\mathbf{g}(\mathbf{x}(t))\right] .
$$

Alle drei Zeitverzögerungsregelungen können für viele experimentelle Situationen verhältnismäßig leicht mit Hilfe von (analoger) Elektronik implementiert werden und sind damit geeignet, auch Chaos, das auf schnellen Zeitskalen auftritt, zu kontrollieren [26]. Abgesehen von der Einführung in [140] wurde NTDAS allerdings bis jetzt nicht in Experimenten angewendet. Aus diesem Grund wird im Zuge dieser Arbeit auch nicht mehr explizit auf NTDAS, sondern hauptsächlich auf (E)TDAS eingegangen werden.

\subsection{Multiple Delay Feedback Control}

Wie bereits erwähnt, ist ETDAS sehr erfolgreich für die Stabilisierung von instabilen periodischen Orbits eingesetzt worden. Für die Stabilisierung von instabilen Fixpunkten $(\dot{\mathbf{x}}=\mathbf{f}(\mathbf{x})=0)$ hingegen ist diese Methode weniger geeignet, was zum Teil daran liegt, dass das Kontrollsignal bereits bei Erreichen eines $\tau$-periodischen Orbits verschwindet. Um diesen Vorgang zu verhindern, müssen weitere Bedingungen an das Regelsignal gestellt werden, die nur durch konstante Lösungen erfüllt werden können. Eine Möglichkeit hierfür besteht darin, einen zweiten Term mit einer weiteren, von $\tau$ verschiedenen Verzögerungszeit zu installieren, was auf das Regelsignal

$$
\mathbf{u}(t)=k[\mathbf{g}(\mathbf{x}(t-\tau))-\mathbf{g}(\mathbf{x}(t))]+\tilde{k}[\mathbf{g}(\mathbf{x}(t-\tilde{\tau}))-\mathbf{g}(\mathbf{x}(t))]
$$

führt. Ist das Verhältnis der Verzögerungszeiten $\tau / \tilde{\tau}$ irrational, so existiert kein periodischer Orbit, bei dem das Kontrollsignal auf Null zurückgeht. Nur für Fixpunkte $\mathbf{x}_{0}$ mit $\mathbf{g}(\mathbf{x}(t))=$ const tragen die Differenzen in Gl. (2.15) nichts zum Kontrollsignal bei, die Regelung ist nichtinvasiv.

Im allgemeinen bietet es sich an, mehr als zwei Verzögerungszeiten sowie unterschiedliche Verstärkungsfaktoren für die zeitverzögerten und nicht zeitverzögerten Anteile des Regelsignals zu verwenden. Dieses Multiple Delay Feedback Control (MDFC) [4-6] führt so zu der Kontrollvorschrift

$$
\begin{aligned}
\mathbf{u}(t)=k_{0}+k_{1 a} \mathbf{g}_{\mathbf{1}}\left(\mathbf{x}\left(t-\tau_{1}\right)\right)-k_{1 b} \mathbf{g}_{\mathbf{1}}(\mathbf{x}(t)) & +\ldots \\
& +k_{M a} \mathbf{g}_{\mathbf{M}}\left(\mathbf{x}\left(t-\tau_{M}\right)\right)-k_{M b} \mathbf{g}_{\mathbf{M}}(\mathbf{x}(t)) .
\end{aligned}
$$


Dabei kommen bis zu $M$ verschiedene Verzögerungszeiten $\tau_{1}, \ldots, \tau_{M}$ zum Einsatz, wobei die Messfunktionen(en) durch $\mathbf{g}_{\mathbf{m}}$ gekennzeichnet sind. Im Gegensatz zu (E)TDAS oder NTDAS müssen die Verzögerungszeiten nicht ganzzahlige Vielfache voneinander sein, sondern können erst einmal frei und unabhängig voneinander gewählt werden. Weiterhin kann der Anteil jedes einzelnen Terms zum Gesamtsignal durch die Parameter $k_{m a}, k_{m b}$ separat gewichtet werden, was im allgemeinen allerdings zunächst zu einer invasiven Regelung führt. Um einen Fixpunkt des ungestörten Systems zu stabilisieren, müssen weitere Randbedingungen erfüllt werden, um die Regelung nichtinvasiv zu gestalten [6]. Das kann entweder durch die Anpassung des konstanten Parameters $k_{0}$ oder durch die Einhaltung der Bedingung

$$
\sum_{m=m_{1}}^{m_{1}+l} k_{m a}-k_{m b}=0
$$

für die Verstärkungsfaktoren $k_{m a}, k_{m b}$ für jede $l$-mal verwendete Messfunktion erreicht werden. Andernfalls wird das Kontrollsignal nicht verschwinden, sondern unter Umständen einen konstanten von Null verschiedenen Wert annehmen und damit einen neuen Fixpunkt generieren. Das mag für bestimmte Anwendungen auch von Interesse sein, steht aber nicht im Vordergrund dieser Arbeit und soll auch im weiteren nicht betrachtet werden. Bei der Verwendung wechselspannungsgekoppelter Eingangssignale (z. B. nach Hochpassfilterung oder Subtraktion eines konstanten Gleichspannungspegels) oder bei der Fixpunktstabilisierung am Ursprung entfällt diese Beschränkung in den Vorfaktoren. Weitere Bedeutung erlangen die individuellen Vorfaktoren $k_{m a}, k_{m b}$ bei der Umgehung von Beschränkungen bezüglich der Stabilisierbarkeit von Fixpunkten, worauf im vierten Kapitel eingegangen werden wird.

Vergleiche über die Güte der Stabilisierung von Fixpunkten mit Hilfe der verschiedenen Regelungsmethoden lassen sich z. B. durch die Größe der Stabilitätsgebiete und mit den absoluten Stabilitätswerten anstellen. Zu diesem Zweck muss eine lineare Stabilitätsanalyse des Vektorfeldes $\mathbf{f}(\mathbf{x})$ am Fixpunkt $\mathbf{x}_{0}$ duchgeführt werden, die auf lokalen Eigenschaften in einer kleinen Umgebung des betrachteten Fixpunktes basiert. Verfügt ein System über mehrere koexistierende Fixpunkte, so entscheiden Bassins und die Wahl der Anfangsbedingungen darüber, zu welchem Fixpunkt die Systemdynamik letztendlich konvergiert. Dementsprechend muss für jeden Fixpunkt eine eigene Stabilitätsanalyse vorgenommen werden.

\subsection{Lineare Stabilitätsanalyse}

Ausgangspunkt für eine solche Stabilitätsanalyse ist ein gegebenes Vektorfeld $\dot{\mathbf{x}}=\mathbf{f}(\mathbf{x})$ mit einem Fixpunkt bei $\mathbf{x}_{0}$. In den weiteren Betrachtungen wird immer stillschweigend von einer additiven Kontrollgröße ausgegangen, was auf

$$
\dot{\mathbf{x}}(t)=\mathbf{f}(\mathbf{x}(t))+\mathbf{u}(t)
$$


führt, wobei das eigentliche Kontrollsignal u durch MDFC aus Gl. (2.16) gegeben ist. Für eine Bewertung der Stabilität ist die zeitliche Entwicklung kleiner Störungen $\mathbf{e}(t)=\mathbf{x}(t)-$ $\mathbf{y}(t)$ ausschlaggebend. Wachsen diese mit der Zeit an, so ist das gesamte rückgekoppelte System instabil und damit bei unveränderter Parameterwahl nicht erfolgreich regelbar. Man erhält demnach für kleine Störungen den Ausdruck

$$
\begin{aligned}
\dot{\mathbf{e}}(t)= & \mathbf{f}(\mathbf{x})-\mathbf{f}(\mathbf{y}) \\
& +\sum_{m=1}^{M}\left[k_{m a} \mathbf{g}_{\mathbf{m}}\left(\mathbf{x}\left(t-\tau_{m}\right)\right)-k_{m b} \mathbf{g}_{\mathbf{m}}(\mathbf{x}(t))\right]-\left[k_{m a} \mathbf{g}_{\mathbf{m}}\left(\mathbf{y}\left(t-\tau_{m}\right)\right)-k_{m b} \mathbf{g}_{\mathbf{m}}(\mathbf{y}(t))\right] .
\end{aligned}
$$

Linearisierung führt schließlich auf

$$
\begin{aligned}
\dot{\mathbf{e}}(t) & =D \mathbf{f}(\mathbf{x}) \mathbf{e}(t) \\
& +\sum_{m=1}^{M} k_{m a} D \mathbf{g}_{\mathbf{m}}\left(\mathbf{x}\left(t-\tau_{m}\right)\right) \mathbf{e}\left(t-\tau_{m}\right)-k_{m b} D \mathbf{g}_{\mathbf{m}}(\mathbf{x}(t)) \mathbf{e}(t)
\end{aligned}
$$

mit den Jakobi-Matrizen $D \mathbf{f}(\mathbf{x})$ und $D \mathbf{g}_{\mathbf{m}}(\mathbf{x})$. Ein Ansatz zur Lösung dieser Differentialgleichung ist durch

$$
\begin{aligned}
\mathbf{e}(t) & =e^{-\lambda t} \mathbf{e}_{0} \\
\mathbf{e}\left(t-\tau_{m}\right) & =e^{-\lambda \tau_{m}} \mathbf{e}(t) .
\end{aligned}
$$

gegeben und führt nach Einsetzen in Gl. (2.20) sowie Betrachtung des Fixpunktes $\mathbf{x}_{0}$ auf

$$
\dot{\mathbf{e}}(t)=\left(D \mathbf{f}\left(\mathbf{x}_{0}\right)+U_{R}\left(\mathbf{x}_{0}\right)\right) \mathbf{e}(t)
$$

mit der Rückkopplungsmatrix

$$
U_{R}\left(\mathbf{x}_{0}, \lambda\right)=\sum_{m=1}^{M}\left(k_{m a} e^{-\lambda \tau_{m}}-k_{m b}\right) D \mathbf{g}_{\mathbf{m}}\left(\mathbf{x}_{0}\right) .
$$

Die Jacobi-Matrix $D \mathbf{f}\left(\mathbf{x}_{0}\right)$ in Gl. (2.22) beschreibt in diesem Zusammenhang die normale lineare Stabilität des ungestörten Systems. Das Kontrollsignal (2.16) wird lediglich durch die zweite Matrix (Rückkopplungsmatrix $U_{R}$ ) berücksichtigt, die alle Details enthält, wie die angewendete Rückkopplung auf die inneren Freiheitsgrade des betrachteten dynamischen Systems wirkt. Für zeitverzögerte Systeme wird also das Eigenwertproblem der Jacobi-Matrix $D \mathbf{f}\left(\mathbf{x}_{0}\right)$ durch das Eigenwertproblem der Matrix $D \mathbf{f}\left(\mathbf{x}_{0}\right)+U_{R}\left(\mathbf{x}_{0}\right)$ mit der charakteristischen Matrix [54]

$$
\Delta(\lambda)=\lambda I-D \mathbf{f}\left(\mathbf{x}_{0}\right)-U_{R}\left(\mathbf{x}_{0}, \lambda\right)
$$


ersetzt. $I$ bezeichne an dieser Stelle die Einheitsmatrix. Viel wichtiger als die charakteristische Matrix ist jedoch deren Determinante det $(\Delta(\lambda))$. Der betrachtete Fixpunkt $\mathbf{x}_{0}$ ist stabil, wenn alle Eigenwerte $\lambda$ der transzendenten charakteristischen Gleichung

$$
\operatorname{det}(\Delta(\lambda))=0
$$

einen negativen Realteil $\operatorname{Re}(\lambda)$ besitzen. Nur dann werden sich kleine Störungen in der zeitlichen Entwicklung reduzieren, der Fixpunkt ist stabil. Was demnach benötigt wird, um die Stabilität des geregelten Systems zu bestimmen, ist der Eigenwert mit dem größten Realteil, denn dieser entspricht gerade der am stärksten instabilen Richtung des betrachteten Fixpunktes. Durch die Anwendung von Zeitverzögerungsregelungen verfügt das Gesamtsystem Gl. (2.18) bzw. Gl. (2.25) (dynamisches System inklusive Regelung) allerdings über unendlich viele Eigenwerte. Glücklicherweise existieren aber nur endlich viele Eigenwerte, deren Realteil größer als eine bestimmte Konstante ist [54], z. B. größer als Null. Um diese endliche Anzahl an Eigenwerten zu finden, wurde zunächst von einem Gitter in der komplexen Ebene ausgegangen, um die Werte zu detektieren, für deren Realund Imaginärteil die charakteristische Gleichung (2.25) gleichzeitig Null oder zumindest möglichst nahe an Null ist. Diese Kombinationen waren wiederum Startwerte für einen gedämpften Newton-Algorithmus, um die genauen Eigenwerte zu ermitteln.

Ich habe MDFC entwickelt und für viele dynamische Systeme in Theorie und Praxis ausprobiert. Dabei hat sich herausgestellt, dass MDFC Pyragas' TDAS und seinen entsprechenden Erweiterungen bezüglich der Stabilisierung von Fixpunkten überlegen ist.

\subsection{Beispiel - der Colpitts-Oszillator}

Eine Illustrierung dieser Feststellung sowie der Wirkungsweise von MDFC soll im folgenden durch den Colpitts-Oszillator vorgenommen werden. Aber die Ergebnisse, die im Anschluss zu Tage gefördert werden, sind nicht auf dieses Beispiel beschränkt, sondern auf viele dynamische Systeme verallgemeinerbar [5, 6]. Der untersuchte Colpitts-Oszillator kann durch die drei Differentialgleichungen

$$
\begin{aligned}
C_{1} \frac{d U_{C_{1}}}{d t} & =-\alpha_{F} f\left(-U_{C_{2}}\right)+I_{L}+\frac{C_{1}}{T} u(t) \\
C_{2} \frac{d U_{C_{2}}}{d t} & =\left(1-\alpha_{F}\right) f\left(-U_{C_{2}}\right)-G_{0} U_{C_{2}}+I_{L}-I_{0} \\
L \frac{d I_{L}}{d t} & =-U_{C_{1}}-U_{C_{2}}-R I_{L}+U_{c c}
\end{aligned}
$$

beschrieben werden und ist ein typisches Beispiel für einen elektronischen Chaos-Oszillator in Form einer rückgekoppelten Basisschaltung (Abb. 2.1). Die Variablen entsprechen zwei Kondensatorspannungen $\left(U_{C_{1}}, U_{C_{2}}\right)$ und dem Spulenstrom $\left(I_{L}\right)$, die mit den Parametern 
$L=91 \mu \mathrm{H}, R=33 \Omega, R_{1}=242 \Omega, C_{1}=$ $68 \mathrm{nF}, C_{2}=68 \mathrm{nF}, I_{s}=14.34 \mathrm{fA}, U_{t}=$ $0.027 \mathrm{~V}, \alpha_{F} \approx 0.99, U_{c c}=5 \mathrm{~V}, G_{0}=0$ und den Zusammenhängen

$$
\begin{aligned}
I_{E} & =f\left(U_{B E}\right)=I_{S}\left(\exp \left(U_{B E} / U_{t}\right)-1\right), \\
I_{0} & =\frac{U_{C_{2}}+U_{c c}}{R_{1}} \\
T & =\sqrt{L \frac{C_{1} C_{2}}{C_{1}+C_{2}}}
\end{aligned}
$$

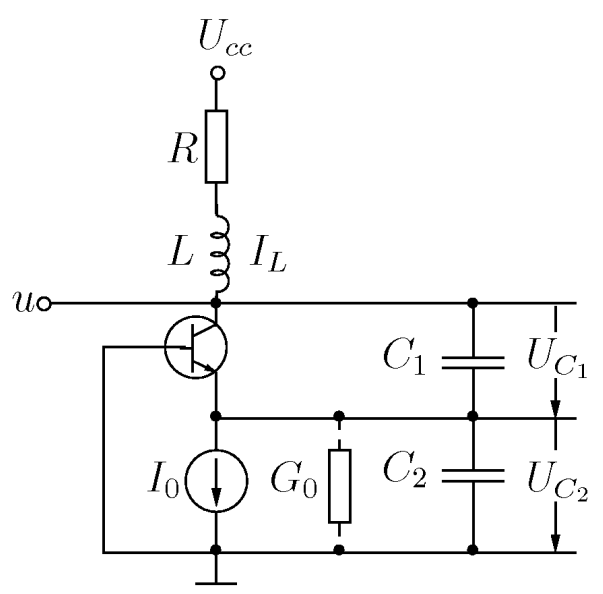

aufgrund der nichtlinearen Kennlinie des verwendeten Transistors chaotische Dynamik für das freilaufende System zeigen $(u(t)=0) . T$ stellt eine typische Zeitskala des Oszillators dar, dessen natürliche Periode wiederum $T_{0}=$ $2 \pi T$ entspricht. Um den systemeigenen Fixpunkt $\mathbf{x}_{0}=\left(U_{C_{1}}, U_{C_{2}}, I_{L}\right)^{t r} \approx$

Abb. 2.1: Schematischer Aufbau des Colpitts-Oszillators (2.26a). Für einen Regeleingriff bieten sich die Spannungen $U_{C_{1}}$ und $U_{C_{2}}$ an. $(5.1759,-0.7502,0.0174)$ zu stabilisieren, wird im folgenden ein Kontrollsignal der Form $\mathbf{u}=(u(t), 0,0)^{t r}$ bestehend aus der Kondensatorspannung $U_{C_{1}}$ und verschwindendem Bias $k_{0}$ gemäß

$$
u(t)=\sum_{m=1}^{M} k_{m a} U_{C_{1}}\left(t-\tau_{m}\right)-k_{m b} U_{C_{1}}(t)
$$

angenommen. Unter Verwendung der Gleichungen (2.26) wird die Rückkopplungsmatrix $U_{R}$ für die lineare Stabilitätsanalyse besonders einfach und lautet

$$
U_{R}=\left(\begin{array}{ccc}
u_{R}(\lambda) & 0 & 0 \\
0 & 0 & 0 \\
0 & 0 & 0
\end{array}\right)
$$

mit

$$
u_{R}(\lambda)=\sum_{m=1}^{M} k_{m a} e^{-\lambda \tau_{m}}-k_{m b} .
$$

Die resultierende charakteristische Matrix

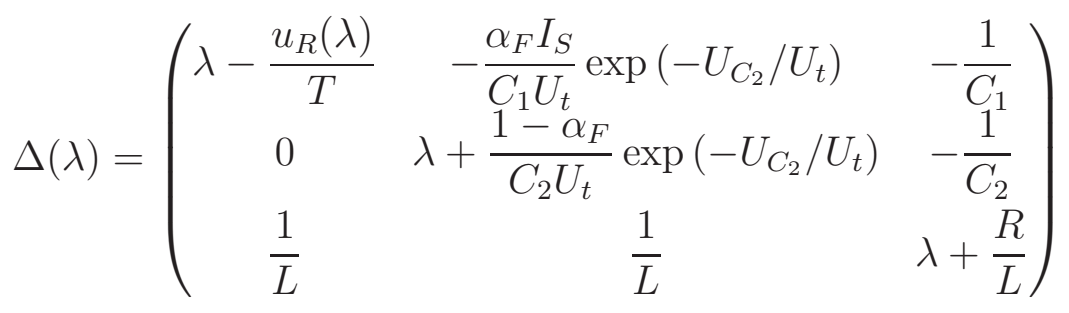


führt schließlich auf die charakteristische Gleichung

$$
\operatorname{det}(\Delta(\lambda))=\left(\lambda-\frac{u_{R}(\lambda)}{T}\right)\left(\left(\lambda+\frac{R}{L}\right)\left(\lambda+s_{2}\right)+\frac{1}{L C_{2}}\right)+\frac{1}{L C_{1}}\left(\lambda+s_{2}\right)+s_{3}=0
$$

mit

$$
s_{2}=\frac{1-\alpha_{F}}{C_{2} U_{t}} \exp \left(-U_{C_{2}} / U_{t}\right), \quad s_{3}=\frac{\alpha_{F} I_{S}}{C_{1} C_{2} U_{t}} \exp \left(-U_{C_{2}} / U_{t}\right) .
$$

Gemäß den Ausführungen auf Seite 19 besteht die Hauptaufgabe nun darin, den Eigenwert mit dem größten Realteil der transzendenten Gleichung (2.30) zu finden. Die Stabilitätsdiagramme aus Abb. 2.2 sind das Ergebnis dieser Berechnungen am Fixpunkt $\mathbf{x}_{0}$ für symmetrische Vorfaktoren $k_{m a}=k_{m b}=k_{m}$. Abb. 2.2a zeigt das resultierende Stabilitätsdiagramm in der $\tau_{1}-\tau_{2}$-Ebene (Aufsicht) für den Fall von nur zwei aktiven Verzögerungszeiten $\left(k_{m}=0, m>2, m \in \mathbb{N}\right)$ und fest eingestellten Verstärkungsfaktoren $k_{1}=k_{2}=1.35$. Regionen mit Kontrollparameterkombinationen, die eine erfolgreiche Stabilisierung des betrachteten Fixpunktes $\mathbf{x}_{0}$ herbeiführen, sind blau und grün gekennzeichnet - je nach der entsprechenden Stabilität. Alle Stabilitätsdiagramme, die in dieser Arbeit gezeigt werden, beginnen mit $\tau_{m} \geq 0.2$, damit auch sicher eine Verzögerung aktiv ist. D. h. die Grenzen $\tau_{1} / T=0, \tau_{2} / T \neq 0$ und $\tau_{2} / T=0, \tau_{1} / T \neq 0$ sind nicht enthalten, können aber dem separaten TDAS-Einzelplot entnommen werden. Aus diesem Grund entspricht TDAS in dieser Abbildung auch nur der Diagonalen $\tau_{1} / T=\tau_{2} / T$. So ist Abb. 2.2a zu entnehmen, dass für $\tau_{1} / T=\tau_{2} / T>2$ keine TDAS-Stabilisierung mehr möglich ist, wobei allerdings mit MDFC und $\tau_{1} / T \neq \tau_{2} / T$ der entsprechende Gleichgewichtszustand für alle Verzögerungszeiten $\tau_{m} / T \in[0.2,14]$ stabilisiert werden kann, wenn Parameterkombinationen aus dem Bereich der farbigen Streifen parallel zur Diagonalen in Abb. 2.2a verwendet werden. Der horizontale Abstand dieser Streifen entspricht ungefähr der Hälfte der natürlichen Periode $T_{0}=2 \pi T$. Im Vergleich zu Abb. 2.2a liefert Abb. 2.2b besser Auskunft über die Güte und Robustheit der Regelung in Form der Stabilitätsfunktion $\max (0,-\operatorname{Re}(\lambda))$ aufgetragen gegen $\tau_{1} / T$ und $\tau_{2} / T$. Dabei bezeichnet $\lambda$ den Eigenwert mit dem größten Realteil $\operatorname{Re}(\lambda)$. Je höher demnach die Spitzen in dem Stabilitätsgebirge sind, desto robuster und sicherer ist auch die Fixpunktstabilisierung. Dementsprechend kennzeichnen die hohen Peaks für die verschiedenen Verzögerungszeiten $\left(\tau_{1} / T \neq \tau_{2} / T\right)$ parallel zur Diagonalen in der $\tau_{1} / T$ - $\tau_{2} / T$-Ebene in Abb. 2.2b MDFC als stabilere Methode zur Fixpunktstabilisierung im Vergleich zu TDAS $\left(\tau_{1}=\tau_{2}\right)$.

Die Verwendung von mehreren Verzögerungszeiten kann allerdings noch weiter ausgenutzt werden. Aufschluss über diesen Sachverhalt geben die Abb. 2.2c,d, die wiederum einen Stabilitätsplot in der $\tau_{1} / T$ - $\tau_{2} / T$-Ebene bei Einsatz von drei Verzögerungszeiten und festen Verstärkungen $k_{1}=k_{2}=k_{3}=1.35$ sowie $\tau_{3} / T=3.1$ zeigen. Wie man hieran sehen kann, sind die farbig markierten Stabilitätsregionen noch weiter ausgedehnt worden beim Übergang von zwei auf drei entsprechend gut gewählte Verzögerungszeiten. Dabei konnte die Stabilität noch weiter auf einen Wert von $\max (0,-\operatorname{Re}(\lambda)) \approx 0.37$ gesteigert werden. Bei Aktivierung einer weiteren Verzögerungszeit mit $\tau_{4} / T=1.32$ werden die Stabilitätsbereiche noch einmal erheblich vergrößert (Abb. 2.2e,f). So ist unter Einsatz moderater 
(a)

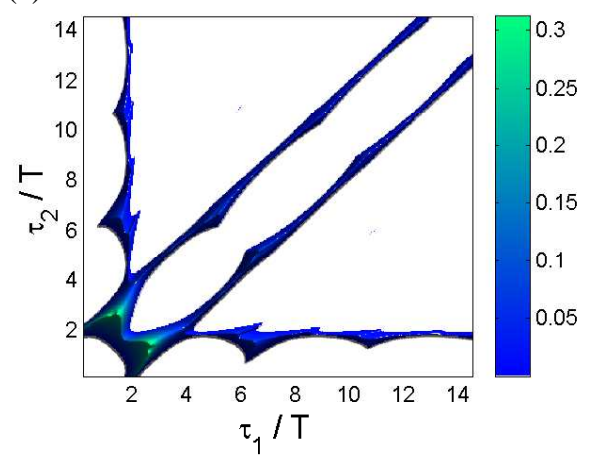

(c)

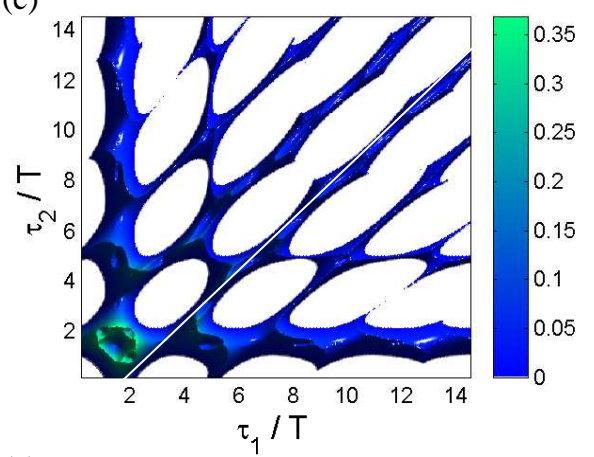

(e)

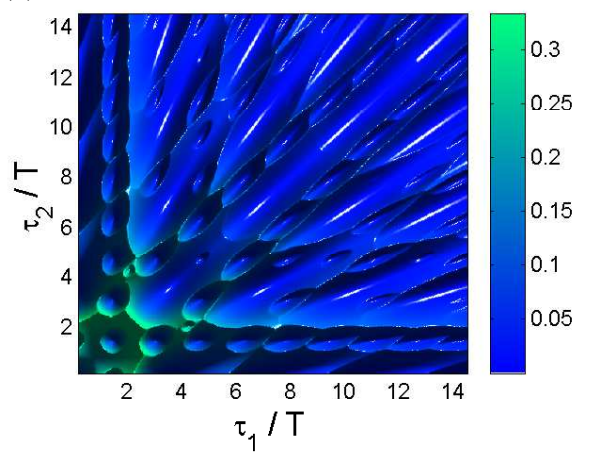

(b)

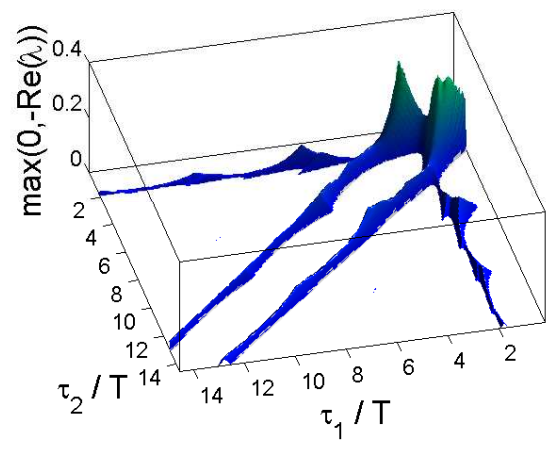

(d)

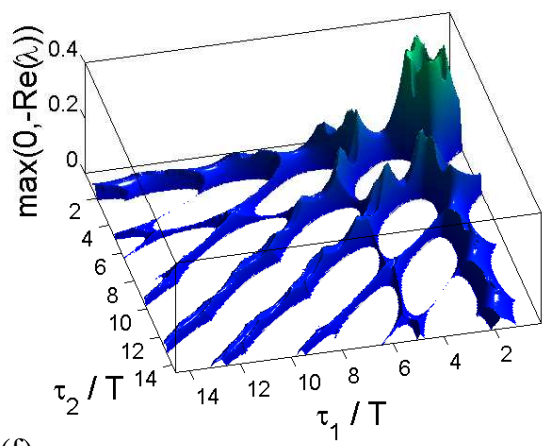

(f)

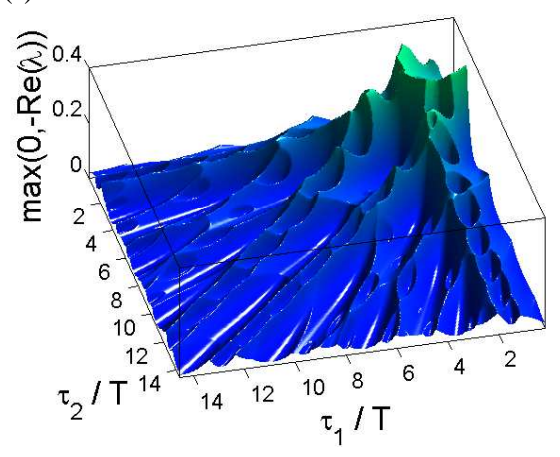

Abb. 2.2: Stabilitätsdiagramme des mit MDFC (2.26b) geregelten ColpittsOszillators (2.26a) unter Einsatz von (a), (b) zwei $\left(k_{m}=1.35\right),(\mathrm{c}),(\mathrm{d})$ drei $\left(k_{m}=1.35, \tau_{3} / T=3.1\right)$ und (e),(f) vier Verzögerungszeiten $\left(k_{m}=1.35, \tau_{3} / T=3.1\right.$, $\left.\tau_{4} / T=1.32\right)$. Die Abbildungen zeigen die Stabilitätsfunktion $\max (0,-\operatorname{Re}(\lambda))$ vs. $\tau_{1} / T$ und $\tau_{2} / T$, wobei $\operatorname{Re}(\lambda)$ den größten Realteil der Eigenwerte der charakteristischen Gleichung (2.30) bezeichnet. Kombinationen der normierten Verzögerungszeiten $\tau_{1} / T$ und $\tau_{2} / T$, die zu einer Fixpunktstabilisierung durch MDFC führen, sind farbig gekennzeichnet. Es sei darauf hingewiesen, dass die Seitenansichten (b),(d),(f) im Vergleich zu den Aufsichten (a),(c),(e) gedreht sind. 
Verstärkungen $\left(k_{1}=k_{2}=k_{3}=k_{4}=1.35\right)$ die ganze $\tau_{1} / T-\tau_{2} / T$ im Bereich bis $\tau / T<14$ geeignet, um den Systemfixpunkt $\mathbf{x}_{0}$ zu stabilisieren.

Abb. 2.3 zeigt für den Fall dreier Verzögerungszeiten die ausgedehnten Stabilitätsgebiete im Parameterraum. Für diese dreidimensionale Projektion wurde die Verstärkung der zweiten und dritten Verzögerungszeit auf $k_{2}=k_{3}=1.35$ sowie $\tau_{3} / T=3.1$ gesetzt. Parameterkombinationen von $\tau_{1} / T, \tau_{2} / T$ und $k_{1}$, die zu einer erfolgreichen Fixpunktstabilisierung führen, sind schwarz gekennzeichnet und zeigen, wie sich die Stabilitätsgebiete in Abhängigkeit von $k_{1}$ ändern. Um die Abhängigkeit der Stabilität von den Verstär-
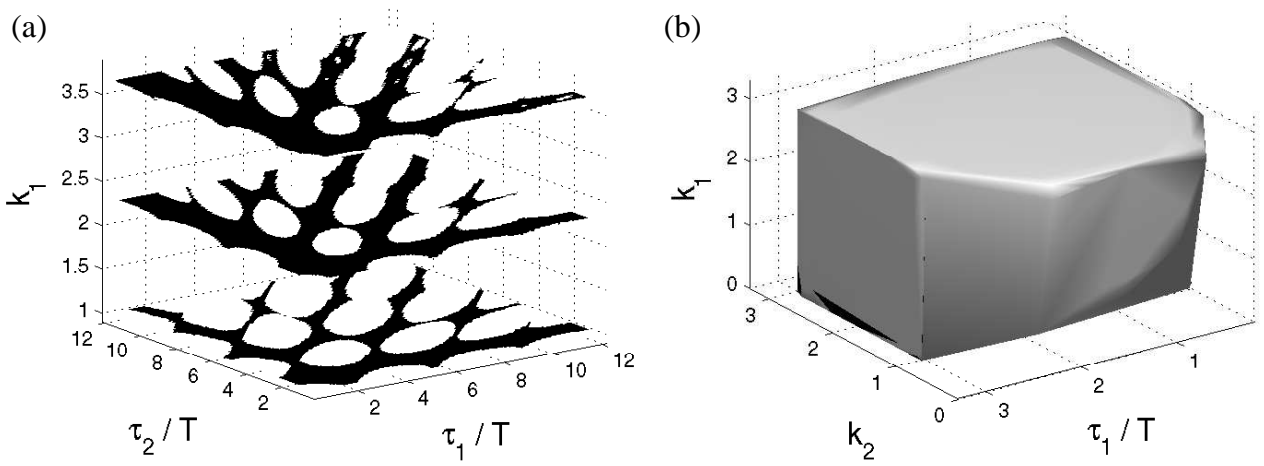

\begin{abstract}
Abb. 2.3: Fixpunktstabilisierung für MDFC aus Gl. (2.26b) unter Verwendung von drei Verzögerungszeiten. (a) Drei Schnitte durch den MDFC-Parameterraum für festes $\tau_{3} / T=3.1$ und $k_{2}=k_{3}=1.35$. Bereiche, die eine Stabilisierung des Fixpunktes des Colpitts-Oszillator (2.26a) herbeiführen, sind schwarz gekennzeichnet. (b) Stabilitätsgebiet vs. $\tau_{1} / T, k_{1}$ und $k_{2}$. Für Fixpunktstabilisierung geeignete Parameter sind grau markiert $\left(\tau_{2} / T=1.8, \tau_{3} / T=3.1\right.$ und $\left.k_{3}=1.35\right)$.
\end{abstract}

kungsfaktoren innerhalb eines Stabilitätszweiges zu untersuchen, wird der Fall gekoppelter Verzögerungszeiten, d.h. $\tau_{1} / T=\tau_{2} / T+1.6$ und $\tau_{3} / T=3.1$ betrachtet. Damit bewegt man sich entlang des weiss gekennzeichneten Stabilitätszweiges der Abb. 2.2c, was zu dem Stabilitätsdiagramm in der $k-\tau_{2} / T$-Ebene aus Abb. 2.4 führt. Die entsprechende Stabilitätsfunktion $\max (0,-\operatorname{Re}(\lambda))$ zeigt neben einer periodischen Modulation ein absolutes Maximum von $\approx-0.4$. Dieses wird bei moderaten Verstärkungsfaktoren erreicht, wobei eine weitere Verstärkungserhöhung über $k>1.5$ die Stabilität wieder mindert.

Die Drehung der Darstellungen aus Abb. 2.2a,c,e und Abb. 2.2b,d,f ist erforderlich, um auch die feinen Strukturen für längere Verzögerungszeiten zu zeigen, da die höchsten Peaks und damit auch die höchsten Stabilitätwerte im Bereich kleiner Verzögerungszeiten zu finden sind. D. h. man sieht in Abb. 2.2b entlang der Diagonalen in Richtung Ursprung im Gegensatz zu Abb. 2.2a. 


\begin{abstract}
Abb. 2.4: Kopplung der Verzögerungszeiten $\tau_{1}$ und $\tau_{2}: k$-Abhängigkeit von MDFC in Form der Stabilitätsfunktion $\max (0,-\operatorname{Re}(\lambda))$ vs. $\tau_{2} / T$ und $k$ entlang der weiss markierten Linie aus Abb. 2.2c. Dabei wird das Regelsignal (2.26b) mit $\tau_{1} / T=\tau_{2} / T+1.6, \tau_{3} / T=3.1, k_{1}=k_{2}=$ $k_{3}=k, k_{4}=0$ angewendet.
\end{abstract}

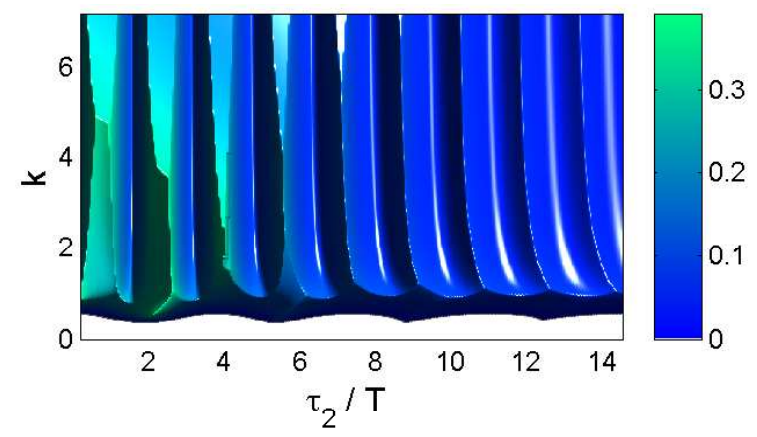

\title{
2.6 Vergleich zu anderen Regelungsmethoden
}

Um die Effizienz und Größe der Stabilitätsgebiete von MDFC zu verdeutlichen, muss ein Vergleich zu anderen (u.a. konventionellen) Methoden zur Fixpunktstabilisierung vorgenommen werden. Für die bekannten zeitverzögerten Regelungen (E)TDAS wird zu diesem Zweck Gl. (2.28) durch den Zusammenhang

$$
\begin{aligned}
u_{R} & =k\left((1-R) e^{-\tau \lambda} \sum_{j=1}^{\infty}\left(R e^{-\tau \lambda}\right)^{j-1}-1\right) \\
& =k\left((1-R) e^{-\tau \lambda} \frac{1}{1-R e^{-\tau \lambda}}-1\right)=k\left(\frac{e^{-\tau \lambda}-1}{1-R e^{-\tau \lambda}}\right)
\end{aligned}
$$

ersetzt werden, der sich aus Gl. (2.11) via Laplace-Transformation ergibt. Für die Simulationen aus Abb. 2.5a,b wurde der Parameter zur Gewichtung der geometrischen Reihe auf einen Wert von $R=0$ (TDAS) bzw. $R=0.7$ (ETDAS) fixiert. Anhand dieser Abbildungen erkennt man, dass der Einsatz von ETDAS (Abb. 2.5b) zu einer Vergrößerung der Stabilitätsgebiete im Vergleich zu TDAS ( $R=0$, Abb. 2.5a) führt. Für längere Verzögerungszeiten ist der Fixpunkt allerdings nur noch schwach stabil, wenn $\tau / T>2$ gewählt wird. MDFC hat diese Problematik nicht und die Fixpunktstabilisierung ist für sehr viel längere Verzögerungszeiten immer noch möglich. Das Resultat für den ETDAS-Grenzübergang $R \rightarrow 1, \tau \rightarrow 0$ ist in Abb. 2.5c dargestellt, die die Stabilitätsfunktion $\max (0,-\operatorname{Re}(\lambda))$ gegenüber der Grenzfrequenz $\omega_{0}$ und dem Verstärkungsfaktor $k$ zeigt. In diesem Fall wird ETDAS zu einem Hochpassfilter mit einem einfachen Pol entsprechend Gl. (2.13), wobei die Eigenwerte in diesem Fall mit Hilfe einer Standard-Stabilitätsanalyse des linearisierten Vektorfeldes $\mathbf{f}(\mathbf{x})$ berechnet werden können. Die Stabilität dieser Rückkopplung (repräsentiert durch den Eigenwert mit dem größten Realteil) ist in Abb. 2.5 farbig skaliert. Je grüner die Farbe desto stabiler ist auch die Rückkopplung für die eingestellten Parameter. Dieser Abb. kann entnommen werden, dass bei entsprechender Parametervariation von $\omega_{0}$ und $k$ die stabilste Regelung einen Wert von ungefähr $\max (0,-\operatorname{Re}(\lambda)) \approx 0.3$ liefert. Das entspricht auch dem Wert, den ETDAS für $R=0.7$ (Abb. 2.5b) herbeiführt und hat damit keine stabilere Regelung zur Folge. Somit ist ETDAS stabiler als TDAS (Minimum: $\operatorname{Re}(\lambda) \approx-0.17)$ aber nicht so stabil wie MDFC mit drei Verzögerungszeiten und 
(a)

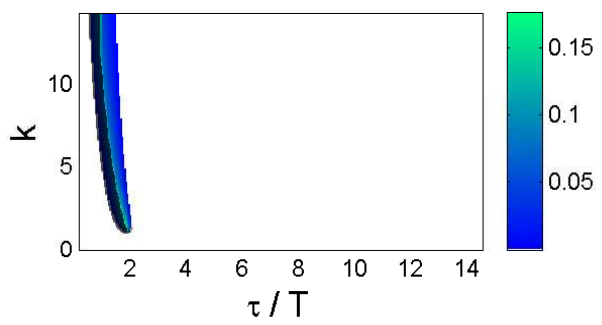

(c)

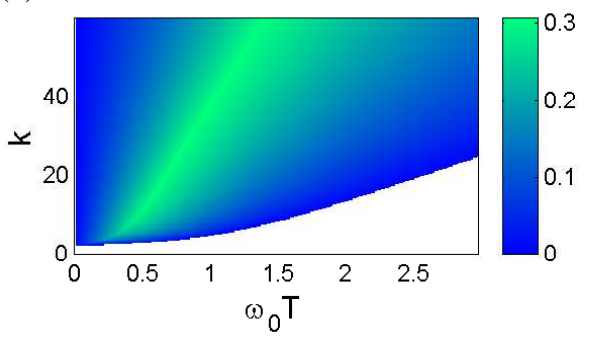

(b)

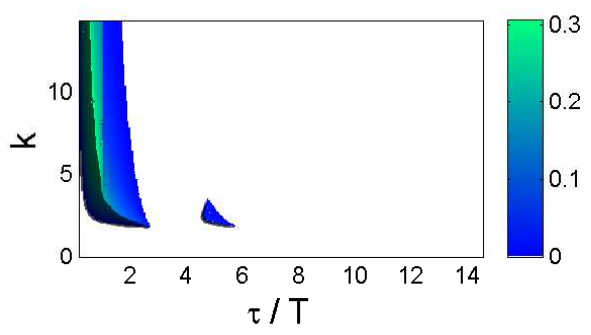

(d)

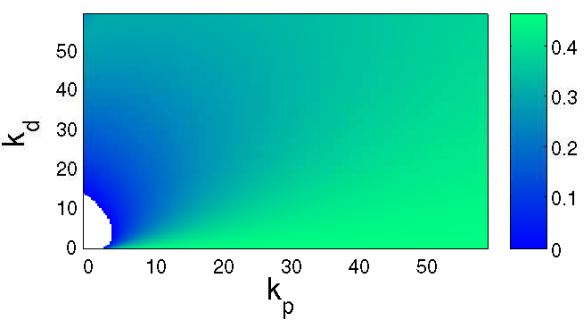

Abb. 2.5: Stabilitätsdiagramme für die Fixpunktstabilisierung des ColpittsOszillators (2.26a) unter Verwendung von (a) TDAS, (b) ETDAS $(R=0.7)$, (c) einem einpoligen Hochpassfilter $\dot{u}(t)=-\omega_{0} u(t)-k \dot{U}_{C_{1}}$ mit Grenzfrequenz $\omega_{0}$ und Verstärkung $k$ sowie (d) einem PD-Regler entsprechend Gl. (2.33). Zur Visualisierung der Ergebnisse wird die Stabilitätsfunktion $\max (0,-\operatorname{Re}(\lambda))$ in Abhängigkeit von den jeweiligen Kontrollparametern verwendet. Bereiche erfolgreicher Fixpunktstabilisierung sind farbig gekennzeichnet, je grüner die Farbe desto stabiler ist die Regelung.

symmetrischen Vorfaktoren (Minimum: $\operatorname{Re}(\lambda) \approx-0.4$ ). Bei Verwendung paarweise verschiedener Verstärkungen $k_{m}$ gemäß $k_{1 a}=k_{1 b}=0.27, k_{2 a}=k_{2 b}=1.56, k_{3 a}=k_{3 b}=0.28$, $\tau_{1} / T=3.613, \tau_{2} / T=2, \tau_{3} / T=3.111$ liefert MDFC einen bei weitem stabileren Wert von $\max (0,-\operatorname{Re}(\lambda)) \approx 0.4767$. Dabei ist allerdings festzustellen, dass die in Abb. $2.2 \mathrm{c}$ vorgestellten Stabilitätsgebiete ihre Symmetrie in der $\tau_{1} / T-\tau_{2} / T$-Ebene eingebüßt haben. Für einen weiteren Vergleich zu konventionellen Regelungmethoden soll nun ein üblicher PD-Regler der Form

$$
u(t)=k_{p} \hat{U}_{C_{1}}+k_{d} \frac{\mathrm{d} \hat{U}_{C_{1}}}{\mathrm{~d} t}
$$

betrachtet werden. Als Eingangssignal dient die Kondensatorspannung $\hat{U}_{C_{1}}$, die aus $U_{C_{1}}$ durch Subtraktion des festen DC-Pegels hervorgeht ${ }^{8}$. Abb. 2.5 d zeigt die farbkodierte Stabilitätsfunktion $\max (0,-\operatorname{Re}(\lambda))$ in Abhängigkeit von den Verstärkungen $k_{p}$ und $k_{d}$ für

\footnotetext{
${ }^{8}$ Das Subtrahieren des festen DC-Pegels anstelle einer Hochpass(AC)-Kopplung des Eingangssignals ist notwendig, um zum einen die Regelung nichtinvasiv zu gestalten und gleichzeitig die Stabilität des PD-Reglers (2.33) nicht schon durch die Stabilitätsfunktion des verwendeten Hochpassfilters (2.13) zu begrenzen.
} 
den Proportional- und den Differenzierungsanteil. Optimale Parameterwahl liefert einen maximalen Stabilitätswert von $\max (0,-\operatorname{Re}(\lambda)) \approx 0.462$. Die Stabilität ist vergleichbar mit der durch MDFC herbeigeführten, allerdings sind die dafür nötigen Verstärkungsfaktoren $k_{p}$ und $k_{d}$ bei weitem höher zu wählen, was wiederum die Effizienz von MDFC verdeutlicht. Erreicht wird diese Effizienz durch eine sehr gute Anpassung der Übertragungsfunktion der Regelung an das Spektrum des Colpitts-Oszillators. Um unter diesem Gesichtspunkt einen intuitiveren Zugang zu der Wirkungsweise von MDFC zu erlangen, ist ein Wechsel in den Fourierraum angebracht.

\subsection{Wirkungsweise im Fourierraum}

Die Fourier-Transformation des Kontrollsignals (2.26b) liefert die Übertragungsfunktion

$$
T(f)=\sum_{m=1}^{M} k_{m a} e^{-i 2 \pi f \tau_{m}}-k_{m b}=\frac{\tilde{u}(f)}{\tilde{U}_{C_{1}}(f)},
$$

die den Ausgang $\tilde{u}$ linear mit dem Eingangssignal $\tilde{U}_{C_{1}}$ verknüpft. Abb. 2.6a zeigt den Absolutwert der Übertragungsfunktion $|T(f)|$ eines einpoligen Hochpassfilters sowie das Frequenzspektrum des Colpitts-Oszillators (2.26a). Um die Auswirkungen zusätzlicher Verzögerungszeiten auf die Übertragungsfunktion von MDFC zu untersuchen, seien die Verstärkungen für jede aktive Verzögerungszeit zu $k_{m a}=k_{m b}=k_{m}=1$ gewählt und der Fall von maximal $M=4$ verschiedenen Verzögerungszeiten betrachtet. So zeigt Abb. 2.6c den Absolutwert der Übertragungsfunktion $|T(f)|$ aus Gl. (2.34) für den Fall einer einzigen Verzögerungszeit $\tau_{1} / T=10$ (durchgezogene Linie, $k_{2}=k_{3}=k_{4}=1$ ), zwei aktiver Verzögerungszeiten (Punkt-Strich-Linie, $\tau_{1} / T=10, \tau_{2} / T=5, k_{3}=k_{4}=0$ ), dreier Verzögerungszeiten (gepunktete Linie, $\tau_{1} / T=16.5, \tau_{2} / T=6, \tau_{3} / T=3.1, k_{4}=0$ ) und vier angewendeter Verzögerungszeiten (gestrichelte Linie, $\tau_{1} / T=10, \tau_{2} / T=5, \tau_{3} / T=3.1$ und $\left.\tau_{4} / T=1.3\right)$. Um einen Fixpunkt zu stabilisieren, muss ein geeignetes Filter eine Kerbe bei $\omega=0$ besitzen [34]. Diese Frequenz (entspricht gerade der eines Fixpunktes) wird dann nicht zurückgekoppelt. Folglich müssen die anderen Kerben der Regelung (2.26b) für $f>0$ so im Fourierraum plaziert werden, dass alle Hauptfrequenzen (entsprechen dominanten, im chaotischen Attraktor eingebetteten instabilen periodischen Orbits) durch das Regelsignal zurückgekoppelt werden. Unter diesem Gesichtspunkt ist es mit der punktgestrichelten Übertragungsfunktion nicht möglich, den systemeigenen Fixpunkt $\mathbf{x}_{0}$ zu stabilisieren, da durch die Wahl von $\tau_{1} / T$ und $\tau_{2} / T$ nicht alle Hauptfrequenzen des Oszillators eliminiert werden. Auf diese Weise wird höchstens ein neuer periodischer Orbit generiert, da das Regelsignal bei der verwendeten Wahl der Verstärkungen $k_{m a}, k_{m b}$ nur im Falle eines Fixpunktes verschwinden würde. Erst wenn die dritte und vierte Verzögerungskette zusätzlich hinzugeschaltet werden (gepunktete und gestrichelte Linie), ist eine erfolgreiche Fixpunktstabilisierung möglich, da alle Kerben nun in unkritische Bereiche des Frequenzraumes verlagert werden. Die entsprechenden Phasen sind in Abb. 2.6d 
(a)

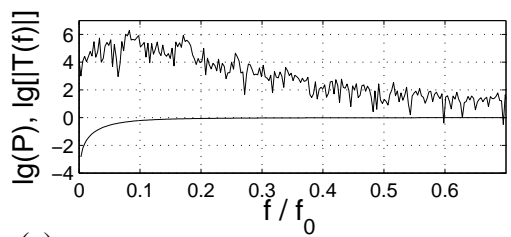

(c)

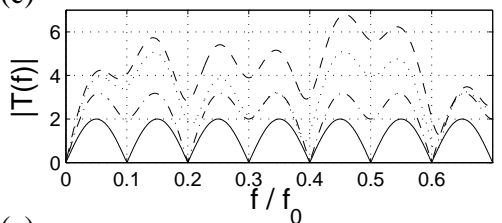

(e)

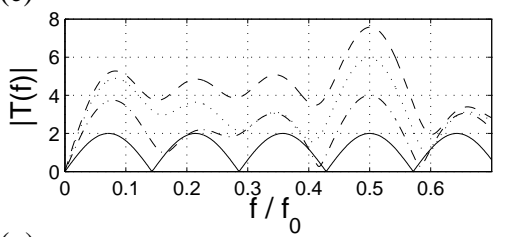

(g)

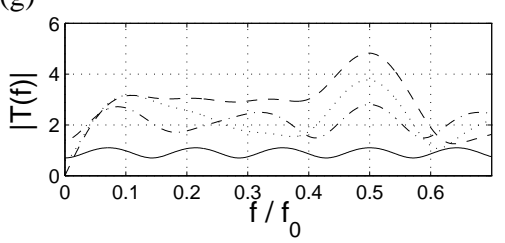

(b)

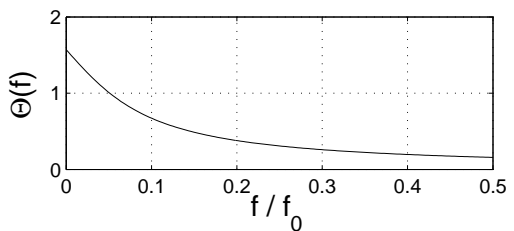

(d)

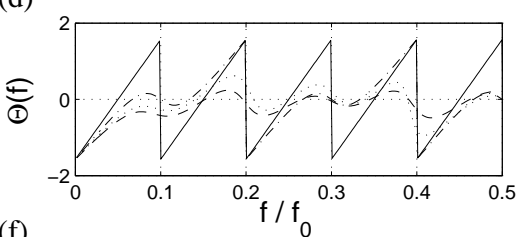

(f)

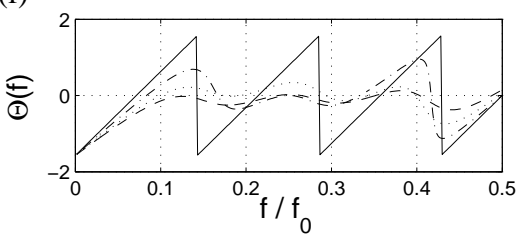

(h)

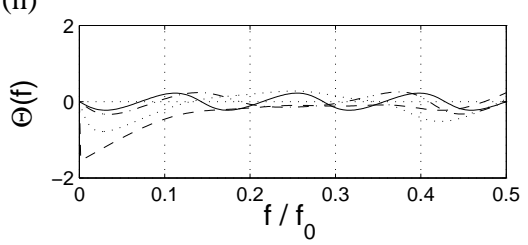

Abb. 2.6: (a) Fourierspektrum des ungeregelten Colpitts-Systems (2.26a) und Übertragungsfunktion eines Hochpassfilters mit der Grenzfrequenz $f_{0}=\omega_{0} /(2 \pi)=0.08$. (b) Zugehöriger Phasenverlauf des Hochpassfilters. (c),(e),(g) Übertragungsfunktionen (2.34) für verschiedene Wahl der Verzögerungszeiten und Verstärkungen: (c) Symmetrische Verstärkungen $k_{m a}=k_{m b}=1$, eine $\left(\tau_{1} / T=10\right.$, durchgezogene Linie), zwei $\left(\tau_{1} / T=10, \tau_{2} / T=5\right.$, Punkt-Strich Linie), drei $\left(\tau_{1} / T=10, \tau_{2} / T=5\right.$, $\tau_{3} / T=3.1$, gepunktete Linie) und vier aktive Verzögerungszeiten $\left(\tau_{1} / T=10\right.$, $\tau_{2} / T=5, \tau_{3} / T=3.1, \tau_{4} / T=1.3$, gestrichelte Linie). Fixpunktstabilisierung ist erst nach der Aktivierung der dritten und vierten Verzögerungszeit möglich, da vorher Kerben $(|T(f)| \approx 0)$ im Bereich der Hauptfrequenzen des ungestörten Systems liegen (vgl. Spektrum aus Abb. 2.6a). (e) Für die Kontrollparameter $k_{m a}=k_{m b}=1$ und $\tau_{1} / T=7$ (durchgezogene Linie), $\tau_{1} / T=7, \tau_{2} / T=5$ (Punkt-Strich Linie), $\tau_{1} / T=7, \tau_{2} / T=5, \tau_{3} / T=3.1$ (gepunktete Linie) sowie $\tau_{1} / T=7, \tau_{2} / T=5$, $\tau_{3} / T=3.1, \tau_{4} / T=1.3$ (gestrichelte Linie) ist eine erfolgreiche Fixpunktstabilisierung nach Aktivierung von zwei oder mehr Verzögerungszeiten möglich, da keine tiefen Kerben in den Bereichen der Hauptfrequenzen des freilaufenden Systems liegen. (g) Gleiche Verzögerungszeiten wie in (e), allerdings mit einer anderen Wahl der Verstärkungsfaktoren: $k_{1 a}=1.3, k_{1 b}=0.7, k_{2 a}=0.4, k_{2 b}=1.0, k_{3 a}=0.8$, $k_{3 b}=0.5, k_{4 a}=0.4, k_{4 b}=0.7$. (d),(f),(h) Die entsprechenden Phasen sind genauso wie die Übertragungsfunktionen aus $(\mathrm{c}),(\mathrm{e}),(\mathrm{g})$ gekennzeichnet. 
dargestellt und weisen für MDFC einen wesentlich glatteren Verlauf als für TDAS (durchgezogene Linie, die ETDAS-Übertragungsfunktion $|T(\omega)|$ unterscheidet sich von TDAS im wesentlichen nur durch die Flankensteilheit, die mit dem Parameter $R$ verknüpft ist) auf.

Ein Beispiel für die Stabilisierung des Fixpunktes $\mathbf{x}_{0}$ mit Hilfe von nur zwei Verzögerungszeiten ist in Abb. 2.6e,f zu sehen, wo durch die Kontrollparameter eine Übertragungsfunktion gegeben ist, die keine Kerben im relevanten Bereich des Frequenzspektrums aufweist. Im Detail handelt es sich bei den Kontrollparametern um $\tau_{1} / T=7$ (durchgezogene Linie, $k_{2}=k_{3}=k_{4}=0$ ) für eine Verzögerungszeit, $\tau_{1} / T=7, \tau_{2} / T=5$ (Punkt-Strich Linie, $k_{3}=k_{4}$, alle Hauptfrequenzen bereits eliminiert) für zwei Verzögerungszeiten, $\tau_{1} / T=7$, $\tau_{2} / T=5, \tau_{3} / T=3.1, k_{4}=0$ (gepunktete Line) im Falle dreier aktiver Verzögerungszeiten sowie um $\tau_{1} / T=7, \tau_{2} / T=5, \tau_{3} / T=3.1$ und $\tau_{4} / T=1.3$ für vier angewendete Verzögerungszeiten. Durch die Aktivierung der dritten und der vierten Verzögerungszeit wird lediglich der Eigenwert mit dem größten Realteil weiter ins Negative verschoben, wodurch der Fixpunkt noch stabiler wird. Die in Abb. 2.6f gezeigten zugehörigen Phasen sind im Bereich $f \in[0.1,0.3]$ mehr oder weniger konstant im Gegensatz zu den auftretenden Phasensprüngen, wenn nur eine Verzögerungszeit aktiviert ist.

Bisher wurden immer alle Vorfaktoren $k_{m a}, k_{m b}$ der Einzelterme gleich (symmetrisch) gewählt. Das ist aber nicht zwingend notwendig, solange für nicht-invasive Kontrolle die vorher schon genannte Nebenbedingung (2.17) berücksichtigt oder der Parameter $k_{0}$ aus Gl. (2.16) geeignet gewählt wird. Ein Beispiel für diesen Sachverhalt ist Abb. 2.6g zu entnehmen, die für dieselben Verzögerungszeiten wie in Abb. 2.6e den Einfluss der geänderten Vorfaktoren $k_{1 a}=1.3, k_{1 b}=0.7, k_{2 a}=0.4, k_{2 b}=1.0, k_{3 a}=0.8, k_{3 b}=0.5, k_{4 a}=$ $0.4, k_{4 b}=0.7$ auf den Betrag der Übertragungsfunktion zeigt. Dabei ist bei Aktivierung aller vier Verzögerungszeiten wiederum eine nicht-invasive Stabilisierung des Fixpunktes gewährleistet. So ist es möglich, MDFC durch geeignete Wahl von $k_{m a}, k_{m b}$ sehr genau und flexibel an die Bedürfnisse der vorherrschenden experimentellen Situation anzupassen [6], flexibler als z. B. (E)TDAS oder einen einpoligen Hochpassfilter (2.13). Diesen
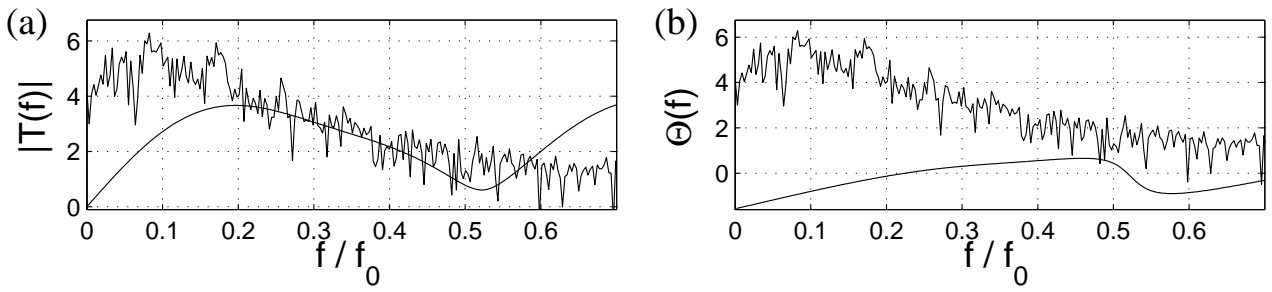

\begin{abstract}
Abb. 2.7: (a) Spektrum des Colpitts-Oszillators sowie eine für $f / f_{0}>0.2$ gut an dieses Spektrum angepasste MDFC-Übertragungsfunktion (2.28) mit den Parametern $k_{a}=k_{1 b}=0.25, k_{2 a}=k_{2 b}=1.58, k_{3 a}=k_{3 b}=0.28, \tau_{1} / T=3.614, \tau_{2} / T=2.0$, $\tau_{3} / T=3.11$. (b) Zugehörige Phase zu diesen Parameterwerten.
\end{abstract}


Sachverhalt spiegelt auch die Wahl der MDFC-Parameter $k_{1}=0.25, k_{2}=1.58, k_{3}=0.28$, $\tau_{1} / T=3.614, \tau_{2} / T=2.0, \tau_{3} / T=3.11$ für hohe Stabilität wider. In diesem Fall zeigt ein Blick auf Abb. 2.7, dass die Übertragungsfunktion von MDFC in einem weiten Bereich sehr genau an das zugrunde liegende Spektrum des Colpitts-Oszillators angepasst ist.

\subsection{Parameterwahl}

\subsubsection{Rückkopplung ohne Totzeit}

Im Gegensatz zur technischen Realisierung ist eine weitere Systemanalyse mehrerer unabhängiger Verzögerungszeiten nur äußerst schwer bzw. abgesehen von Spezialfällen unmöglich ${ }^{9}$, da es im Gegensatz zu dem Fall nur einer Verzögerungszeit $\tau$ keine vergleichbaren Lambert-Funktionen gibt, mit denen weitere theoretische Erkenntnisse wie in [58] gewonnen werden können. Weiterhin ist die in [35] vorgeschlagene adaptive Methode zur Einstellung der Verzögerungszeiten nicht ohne weiteres auf mehrere Verzögerungszeiten verallgemeinerbar. Von daher gestaltet sich das Auffinden optimaler Parameterwerte für MDFC schwierig. Neben den optimalen Parametern sind oft aber auch generell geeignete Werte von Interesse. Aber selbst unter diesem Gesichtspunkt ist bei mehreren unabhängigen Verzögerungszeiten eine analytische Behandlung (relativ) einfacher Systeme wie dem Colpitts-Oszillator (2.26) nicht mehr möglich. Von daher sei im weiteren Verlauf der harmonische Oszillator

$$
\begin{aligned}
& \dot{x}=y \\
& \dot{y}=-d y-\omega_{0}^{2} x+u
\end{aligned}
$$

als einfachstes Beispiel eines dynamischen Systems $\dot{\mathbf{x}}=\mathbf{f}(\mathbf{x})$ betrachtet. $\omega_{0}$ bezeichnet hierbei die Eigenfrequenz des Oszillators mit den Systemvariablen $x, y$. Die Instabilität zum Simulieren einer sich vom Fixpunkt $\mathbf{x}_{0}=(x, y)^{t r}=(0,0)$ entfernenden Dynamik (für $u(t)=0)$ wird durch die negative Dämpfung $d<0$ realisiert. Um diesen Systemfixpunkt $\mathbf{x}_{0}$ zu stabilisieren, wird das MDFC-Signal

$$
u(t)=\sum_{m=1}^{M} k_{m a} x\left(t-\tau_{m}\right)-k_{m b} x(t)
$$

mit dem Regelvektor $\mathbf{u}=(0, u(t))^{t r}$ angewendet. Da $x(t)$ trotz der durch die Instabilität bedingt anwachsenden Amplitude eine harmonische Größe ist, kann Gl. (2.35) mit

\footnotetext{
${ }^{9}$ Aufgrund der folgenden zum Teil etwas mühsamen mathematischen Berechnungen sei dem Leser empfohlen, diesen Abschnitt beim ersten Lesen u. U. lediglich zu überfliegen.
} 
aktivierter Regelung $(\mathbf{u} \neq 0)$ als getriebener harmonischer Oszillator

$$
\begin{aligned}
& \dot{x}(t)=y(t) \\
& \dot{y}(t)=-d y(t)-\left(\omega_{0}^{2}+\sum_{m=1}^{M} k_{m b}\right) x(t)+\sum_{m=1}^{M} k_{m a} x\left(t-\tau_{m}\right)
\end{aligned}
$$

mit der Resonanzfrequenz $\omega_{r}=\sqrt{\omega_{c}^{2}-d^{2} / 2}$ betrachtet werden. $\omega_{c}$ ist die durch die Kontrolle beeinflusste Größe $\omega_{c}^{2}=\omega_{0}^{2}+\sum_{m=1}^{M} k_{m b}>0$, die sich nach der Trennung von zeitverzögerten und nicht zeitverzögerten Termen in Gl. (2.37) ergibt. Die das neue Gesamtsystem (harmonischer Oszillator inklusive aktivierter Regelung) beschreibende Übertragungsfunktion lautet [85]

$$
T(\lambda)=\frac{H(\lambda) C(\lambda)}{1+H(\lambda) C(\lambda)}
$$

mit der Übertragungsfunktion

$$
H(\lambda)=\frac{1}{\lambda^{2}+d \lambda+\omega_{c}^{2}}
$$

des Oszillators und der Übertragungsfunktion

$$
C(\lambda)=\sum_{m=1}^{M} k_{m a} e^{-\lambda \tau_{m}}
$$

der Regelung, die sich jeweils nach Laplace-Transformation von Gl (2.37) und (2.36) ergeben. Um die Stabilität des rückgekoppelten Systems zu determinieren, kann z. B. der Nyquist-Ausdruck

$$
N(i \omega)=-H(i \omega) C(i \omega)=-\frac{\sum_{m=1}^{M} k_{m a} e^{-i \omega \tau_{m}}}{\omega_{c}^{2}-\omega^{2}+i d \omega} .
$$

untersucht werden, woraus sich Bedingungen an ein für Fixpunktstabilisierung erfolgreiches Regelsignal herleiten lassen. Zu diesem Zweck wird die Phase des Nyquist-Ausdruckes (2.41) betrachtet. Für deren Berechnung muss zunächst Gl. (2.41) gemäß $N(i \omega)=\gamma(a+i b)$ mit $\gamma=1 /\left(\left(\omega_{c}^{2}-\omega^{2}\right)^{2}+d^{2} \omega^{2}\right)$ zerlegt werden. Die benötigte Phase

$$
\Theta=\arctan \left(\frac{b}{a}\right)
$$

ergibt sich schließlich aus den Ausdrücken

$$
b=-\left(\omega^{2}-\omega_{c}^{2}\right)\left(\sum_{m=1}^{M} k_{m a} \sin \left(\omega \tau_{m}\right)\right)+d \omega\left(\sum_{m=1}^{M} k_{m a} \cos \left(\omega \tau_{m}\right)\right)
$$


und

$$
a=\left(\omega^{2}-\omega_{c}^{2}\right)\left(\sum_{m=1}^{M} k_{m a} \cos \left(\omega \tau_{m}\right)\right)+d \omega\left(\sum_{m=1}^{M} k_{m a} \sin \left(\omega \tau_{m}\right)\right) .
$$

Im Bereich der Frequenz $\omega_{c}$ (d.h. $\left.\omega^{2}-\omega_{c}^{2} \approx 0\right)$ üben die Terme, die proportional zur Entdämpfung $d$ sind, den größten Einfluss aus, was in der Phase

$$
\Theta_{1} \approx \arctan \left(\frac{\sum_{m=1}^{M} k_{m a} \cos \left(\omega \tau_{m}\right)}{\sum_{m=1}^{M} k_{m a} \sin \left(\omega \tau_{m}\right)}\right), \quad \omega \approx \omega_{c}
$$

resultiert. Mit dem Additionstheorem [31]

$$
\arctan (\alpha)+\arctan \left(\frac{1}{\alpha}\right)= \begin{cases}\frac{\pi}{2} & \alpha>0 \\ -\frac{\pi}{2} & \alpha<0\end{cases}
$$

kann dieser Ausdruck in

$$
\Theta_{1}= \pm \frac{\pi}{2}-\arctan \left(\frac{\sum_{m=1}^{M} k_{m a} \sin \left(\omega \tau_{m}\right)}{\sum_{m=1}^{M} k_{m a} \cos \left(\omega \tau_{m}\right)}\right)
$$

umgewandelt werden. In den Frequenzbereichen, die weit von der Fequenz $\omega_{c}$ entfernt sind, berechnet sich die Phase der Gl. (2.41) zu

$$
\Theta_{2} \approx-\arctan \left(\frac{\sum_{m=1}^{M} k_{m a} \sin \left(\omega \tau_{m}\right)}{\sum_{m=1}^{M} k_{m a} \cos \left(\omega \tau_{m}\right)}\right), \quad 0<\omega<\omega_{c}, \omega>\omega_{c}
$$

die sich von $\Theta_{1}$ lediglich um einen konstanten Versatz von $\mp \pi / 2$ unterscheidet, ansonsten aber äquivalent ist. Von daher wird für die weitere Analyse nur noch Gl. (2.48) betrachtet. Für die Stabilität von $N(i \omega)$ sind die Frequenzen entscheidend, bei denen $N(i \omega)=-1$ bzw. die Phase den Wert $-\pi$ erreicht, wodurch das zurückgekoppelte System instabil wird [3]. Dieser Aspekt liefert für $n \in \mathbb{N}$ die Zusammenhänge

$$
\begin{gathered}
-\pi+\arctan \left(\frac{\sum_{m=1}^{M} k_{m a} \sin \left(\omega \tau_{m}\right)}{\sum_{m=1}^{M} k_{m a} \cos \left(\omega \tau_{m}\right)}\right)=-(2 n+1) \pi, \quad 0<\omega<\omega_{r}, \\
-2 \pi+\arctan \left(\frac{\sum_{m=1}^{M} k_{m a} \sin \left(\omega \tau_{m}\right)}{\sum_{m=1}^{M} k_{m a} \cos \left(\omega \tau_{m}\right)}\right)=-(2 n+1) \pi, \quad \omega>\omega_{r},
\end{gathered}
$$

bzw. unter Ausnutzung der Umkehrfunktion

$$
\frac{\sum_{m=1}^{M} k_{m a} \sin \left(\omega \tau_{m}\right)}{\sum_{m=1}^{M} k_{m a} \cos \left(\omega \tau_{m}\right)}=\tan (2 n \pi)=0, \quad 0<\omega<\omega_{r},
$$




$$
\frac{\sum_{m=1}^{M} k_{m a} \sin \left(\omega \tau_{m}\right)}{\sum_{m=1}^{M} k_{m a} \cos \left(\omega \tau_{m}\right)}=\tan ((2 n+1) \pi)=0, \quad \omega>\omega_{r} .
$$

Dies wiederum impliziert als notwendige Bedingung sowohl für $0<\omega<\omega_{r}$ als auch für $\omega>\omega_{r}$ die Bedingung

$$
\sum_{m=1}^{M} k_{m a} \sin \left(\omega_{s} \tau_{m}\right)=0 .
$$

Für diese bei fest eingestellten Kontrollparametern $k_{m a}, k_{m b}$ und $\tau_{m}$ zu berechnenden kritischen Frequenzen $\omega_{s}$ muss ein stabiles System gewährleistet sein, was $\left|N\left(i \omega_{s}\right)\right|<1$ erfordert und zu

$$
\begin{aligned}
\left|H\left(i \omega_{s}\right) C\left(i \omega_{s}\right)\right| & =\left|\frac{\sum_{m=1}^{M} k_{m a} e^{-i \omega_{s} \tau_{m}}}{\omega_{c}^{2}-\omega_{s}^{2}+i d \omega_{s}}\right|=\frac{\left|\sum_{m=1}^{M} k_{m a}\left(\cos \left(\omega_{s} \tau_{m}\right)-i \sin \left(\omega_{s} \tau_{m}\right)\right)\right|}{\sqrt{\left(\omega_{c}^{2}-\omega_{s}^{2}\right)^{2}+\left(d \omega_{s}\right)^{2}}} \\
& =\frac{\left|\sum_{m=1}^{M} k_{m a} \cos \left(\omega_{s} \tau_{m}\right)\right|}{\sqrt{\left(s_{2}-\omega_{s}^{2}\right)^{2}+s_{3}^{2}}}<\frac{\sum_{m=1}^{M}\left|k_{m a}\right|}{\sqrt{\left(s_{2}-\omega_{s}^{2}\right)^{2}+s_{3}^{2}}}<1
\end{aligned}
$$

für $0<\omega<\omega_{r}$ sowie

$$
\left|H\left(i \omega_{s}\right) C\left(i \omega_{s}\right)\right|=\frac{\left|\sum_{m=1}^{M} k_{m a} \cos \left(\omega_{s} \tau_{m}\right)\right|}{\sqrt{\left(\omega_{s}^{2}-s_{2}\right)^{2}+s_{3}^{2}}}<\frac{\sum_{m=1}^{M}\left|k_{m a}\right|}{\sqrt{\left(\omega_{s}^{2}-s_{2}\right)^{2}+s_{3}^{2}}}<1
$$

für $\omega>\omega_{r}$ mit $s_{2}=\left(2 \omega_{c}^{2}-d^{2}\right) / 2$ und $s_{3}^{2}=d^{2}\left(4 \omega_{c}^{2}-1\right) / 4$ führt. An dieser Stelle wurde bereits ausgenutzt, dass die Summe über die Sinusterme in Gl. (2.52a) nach Einsetzen der kritischen Frequenzen wegfällt. Eine weitere exakte Analyse der Gleichungen $(2.50 \mathrm{a}, \mathrm{b})$ und $(2.52 \mathrm{a}, \mathrm{b})$ ist allerdings wegen der Transzendenz der Gleichungen nicht möglich. Von daher wird sich im weiteren Verlauf der Betrachtungen auf den Fall zweier unterschiedlicher Verzögerungszeiten $\tau_{1}, \tau_{2}$ mit gleichen symmetrischen Verstärkungen $k_{m a}=k_{m b}=k / 2, m \in\{1,2\}$ beschränkt. Nach Anwendung des Additionstheorems [31]

$$
\frac{\sin \left(\omega_{s} \tau_{1}\right)+\sin \left(\omega_{s} \tau_{2}\right)}{\cos \left(\omega_{s} \tau_{1}\right)+\cos \left(\omega_{s} \tau_{2}\right)}=\tan \left(\frac{\omega_{s} \tau_{1}+\omega_{s} \tau_{2}}{2}\right)
$$

verändern sich die Gl. (2.49a,b) zu

$$
\begin{gathered}
-\pi+\frac{\omega_{s}\left(\tau_{1}+\tau_{2}\right)}{2}=-(2 n+1) \pi, \quad 0<\omega<\omega_{r}, \\
-2 \pi+\frac{\omega_{s}\left(\tau_{1}+\tau_{2}\right)}{2}=-(2 n+1) \pi, \quad \omega>\omega_{r},
\end{gathered}
$$

was für den betrachteten MDFC Spezialfall (zwei Verzögerungszeiten, symmetrische Vorfaktoren) die gesuchten kritischen Frequenzen

$$
\begin{aligned}
\omega_{s} & =\frac{4 n \pi}{\tau_{1}+\tau_{2}}, \quad 0<\omega<\omega_{r} \\
\omega_{s} & =\frac{2(2 n+1) \pi}{\tau_{1}+\tau_{2}}, \quad \omega>\omega_{r}
\end{aligned}
$$


durch Umstellen von Gl. (2.54a,b) nach sich zieht. Die Vereinigung der Gleichungen $(2.52 \mathrm{a}, \mathrm{b})$ hat unter Verwendung von $k^{2}>s_{3}^{2}$ für die kritischen Frequenzen (2.55) wiederum

$$
\frac{4 n \pi}{\sqrt{s_{2}-\sqrt{k^{2}-s_{3}^{2}}}}<\tau_{1}+\tau_{2}<\frac{2(2 n+1) \pi}{\sqrt{s_{2}+\sqrt{k^{2}-s_{3}^{2}}}}
$$

zur Folge $\left(k^{2}>s_{3}^{2}\right)$, wonach es für erfolgreiches MDFC zunächst nur auf die Summe der beiden Verzögerungszeiten $\tau_{1,2}$ ankommt. Für den Extremfall der Gleichheit beider Seiten in Gl. (2.56) berechnet sich die Verstärkung zu

$$
|k|=\sqrt{s_{3}^{2}+\left(\frac{4(4 n+1) s_{2}}{32 n^{2}+16 n+4}\right)^{2}} .
$$

Wird anstelle von MDFC aus Gl. (2.36) das (E)TDAS-Signal

$$
u(t)=k\left[(1-R) \sum_{j=1}^{\infty} R^{j-1} x(t-j \tau)-x(t)\right]
$$

mit $\omega_{c}=\omega_{0}^{2}+k>0$ verwendet, so muss die Übertragungsfunktion (2.40) durch

$$
C(\lambda)=k(1-R) \sum_{j=1}^{\infty} R^{j-1} e^{-j \lambda \tau}
$$

ersetzt werden, was nach analogem Vorgehen wie für MDFC die Gleichungen

$$
\begin{gathered}
-\pi+\arctan \left(\frac{\sum_{j=1}^{\infty} R^{j} \sin (j \omega \tau)}{\sum_{j=1}^{\infty} R^{j} \cos (j \omega \tau)}\right)=-(2 n+1) \pi, \quad 0<\omega<\omega_{r}, \\
-2 \pi+\arctan \left(\frac{\sum_{j=1}^{\infty} R^{j} \sin (j \omega \tau)}{\sum_{j=1}^{\infty} R^{j} \cos (j \omega \tau)}\right)=-(2 n+1) \pi, \quad \omega>\omega_{r}
\end{gathered}
$$

liefert. Aus diesen gehen unter Beachtung von $|R|<1$ und Anwendung der Additionstheoreme [31]

$$
\begin{aligned}
& \sum_{j=1}^{N-1} R^{j} \sin (j \omega \tau)=\frac{R \sin (\omega \tau)\left(1-R^{N} \cos (N \omega \tau)\right)-(1-R \cos (\omega \tau)) R^{N} \sin (N \omega \tau)}{1-2 R \cos (\omega \tau)+R^{2}} \\
& \sum_{j=1}^{N-1} R^{j} \cos (j \omega \tau)=\frac{(1-R \cos (\omega \tau))\left(1-R^{N} \cos (N \omega \tau)\right)+R^{N+1} \sin (\omega \tau) \sin (N \omega \tau)}{1-2 R \cos (\omega \tau)+R^{2}}
\end{aligned}
$$


für den Grenzfall $\lim _{N \rightarrow \infty}$ die kritischen Frequenzen

$$
\begin{aligned}
& \omega_{s}=\frac{2 n \pi}{\tau}, \quad 0<\omega<\omega_{r} \\
& \omega_{s}=\frac{(2 n+1) \pi}{\tau}, \quad \omega>\omega_{r},
\end{aligned}
$$

für (E)TDAS hervor. An dieser Stelle sei der Vollständigkeit halber darauf hingewiesen, dass NTDAS über dieselben kritischen Frequenzen $\omega_{s}$ verfügt, die wie folgt zusammengefasst werden können:

\begin{tabular}{c|c|c|c|c} 
& TDAS $\left(\omega_{s}\right)$ & $\operatorname{ETDAS}\left(\omega_{s}\right)$ & $\operatorname{NTDAS}\left(\omega_{s}\right)$ & $\operatorname{MDFC}\left(\omega_{s}\right)$ \\
\hline $0<\omega<\omega_{r}$ & $\frac{2 n \pi}{\tau}$ & $\frac{2 n \pi}{\tau}$ & $\frac{2 n \pi}{\tau}$ & $\frac{4 n \pi}{\tau_{1}+\tau_{2}}$ \\
$\omega>\omega_{r}$ & $\frac{(2 n+1) \pi}{\tau}$ & $\frac{(2 n+1) \pi}{\tau}$ & $\frac{(2 n+1) \pi}{\tau}$ & $\frac{2(2 n+1) \pi}{\tau_{1}+\tau_{2}}$
\end{tabular}

Durch analoge Vorgehensweise wie für MDFC mit zwei Verzögerungszeiten ergibt sich für die Betrachtung des Betrages der Nyquist-Funktion

$$
\left|H\left(i \omega_{s}\right) C\left(i \omega_{s}\right)\right|=k(1-R) \frac{\left|\sum_{j=1}^{\infty} R^{j} \cos \left(j \omega_{s} \tau\right)\right|}{\sqrt{\left(s_{2}-\omega_{s}^{2}\right)^{2}+s_{3}^{2}}}=\frac{s_{4}}{\sqrt{\left(s_{2}-\omega_{s}^{2}\right)^{2}+s_{3}^{2}}}<1
$$

im Bereich $0<\omega<\omega_{0}$ bzw. für $\omega>\omega_{0}$

$$
\left|H\left(i \omega_{s}\right) C\left(i \omega_{s}\right)\right|=k(1-R) \frac{\left|\sum_{j=1}^{\infty} R^{j} \cos \left(j \omega_{s} \tau\right)\right|}{\sqrt{\left(\omega_{s}^{2}-s_{2}\right)^{2}+s_{3}^{2}}}=\frac{s_{4}}{\sqrt{\left(\omega_{s}^{2}-s_{2}\right)^{2}+s_{3}^{2}}}<1
$$

unter Verwendung von

$$
s_{4}=k(1-R) \frac{1-R \cos \left(\omega_{c} \tau\right)}{1-2 R \cos \left(\omega_{c} \tau\right)+R^{2}} .
$$

Mit Hilfe der kritischen Frequenzen $\omega_{s}=2 n \pi / \tau$, bzw. $\omega_{s}=(2 n+1) \pi / \tau$ stellen sich schließlich für den Ausdruck $s_{4}$ mit $k_{r}=k(1-R) /(1+R)$ für ETDAS die zwei Fälle

$$
s_{4}= \begin{cases}k & : \omega_{s}=2 n \pi / \tau \\ k_{r} & : \omega_{s}=(2 n+1) \pi / \tau\end{cases}
$$

ein, die für TDAS $(R=0)$ identisch sind (d.h. $k_{r}=k$ ). Folglich ergibt sich für ETDAS analog zu Gl. (2.55), (2.56) als Bedingung für die Verzögerungszeit $\tau$ der Zusammenhang

$$
\frac{2 n \pi}{\sqrt{s_{2}-\sqrt{s_{4}^{2}-s_{3}^{2}}}}<\tau<\frac{(2 n+1) \pi}{\sqrt{s_{2}+\sqrt{s_{4}^{2}-s_{3}^{2}}}}
$$



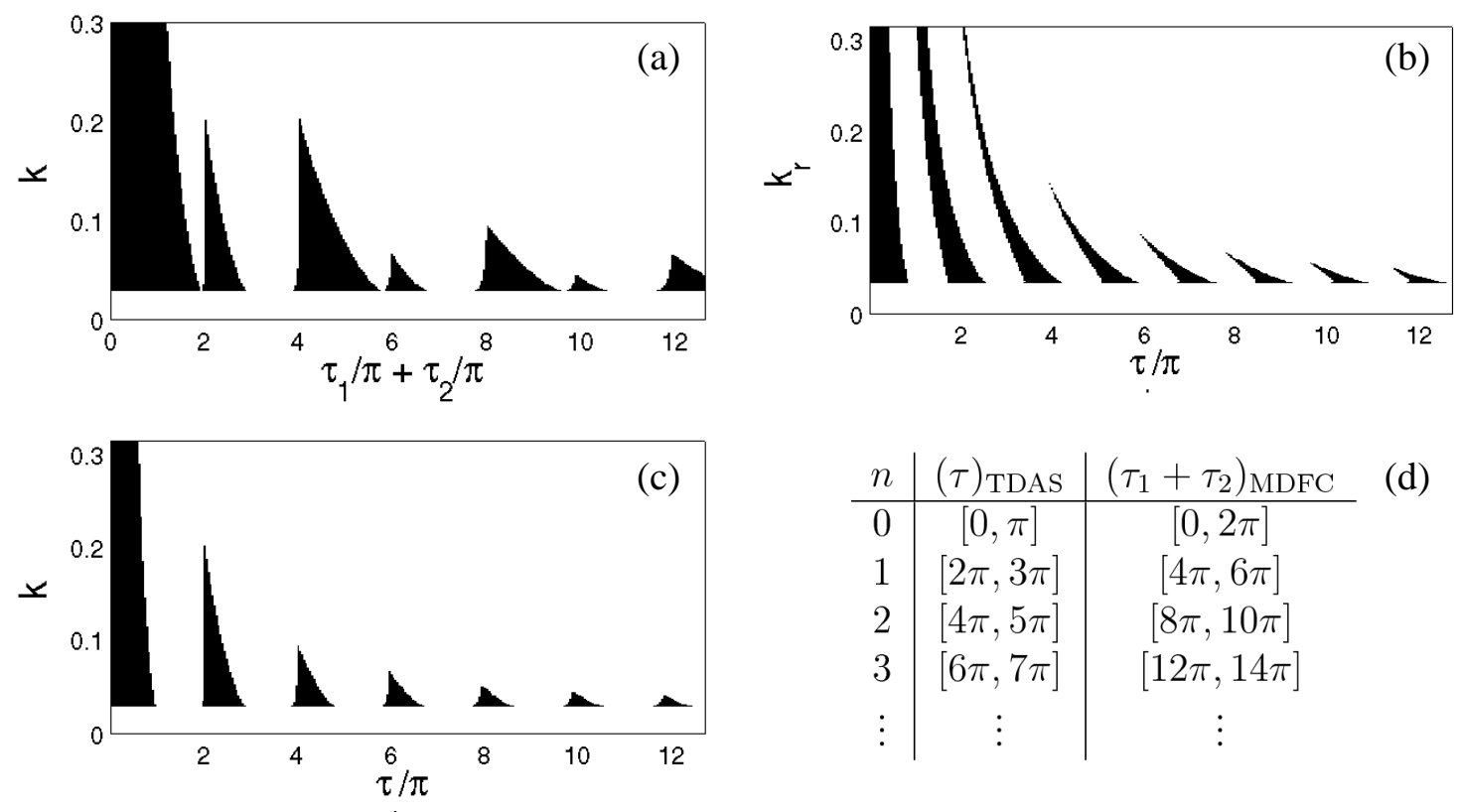

\begin{tabular}{c|c|c}
$n$ & $(\tau)_{\mathrm{TDAS}}$ & $\left(\tau_{1}+\tau_{2}\right)_{\mathrm{MDFC}}$ \\
\hline 0 & {$[0, \pi]$} & {$[0,2 \pi]$} \\
1 & {$[2 \pi, 3 \pi]$} & {$[4 \pi, 6 \pi]$} \\
2 & {$[4 \pi, 5 \pi]$} & {$[8 \pi, 10 \pi]$} \\
3 & {$[6 \pi, 7 \pi]$} & {$[12 \pi, 14 \pi]$} \\
$\vdots$ & $\vdots$ & $\vdots$
\end{tabular}

Abb. 2.8: Stabilitätsgebiete (schwarz markiert) des harmonischen Oszillators (2.35) in Abhängigkeit von der Verstärkung $k$ und der (den) Verzögerungszeit(en) $\tau$ bzw. $\tau_{1}+\tau_{2}$ für (a) MDFC mit zwei Verzögerungszeiten und gleichen Verstärkungen $k / 2$ sowie $\tau$ für (b) ETDAS mit $R=0.7$ und (c) TDAS. Die Verstärkung in (b),(c) ist zu $k$ gewählt, die Parameter aus Gl. (2.35) sind auf $\omega_{0}^{2}=1$ und $d=-0.1$ fixiert. (d) TDAS und MDFC im Vergleich: geeignete Intervalle der Verzögerungszeiten für die Stabilisierung des Fixpunktes des harmonischen Oszillators (2.35).

der für $|R|<1$ reelle Werte garantiert und sich für TDAS zu

$$
\frac{2 n \pi}{\sqrt{s_{2}-\sqrt{k^{2}-s_{3}^{2}}}}<\tau<\frac{(2 n+1) \pi}{\sqrt{s_{2}+\sqrt{k^{2}-s_{3}^{2}}}}
$$

vereinfacht. Der besondere Fall der Gleichheit der Ausdrücke zu beiden Seiten von $\tau$ in den Gl. (2.66), (2.67) liefert als Ergebnis für die Verstärkung im Fall von ETDAS

$$
|k|=\sqrt{\left(\frac{(1+R) s_{3}}{1-R}\right)^{2}+\left(\frac{(1+R)(4 n+1) s_{2}}{(1-R)\left(8 n^{2}+4 n+1\right)}\right)^{2}},
$$

bzw. für TDAS mit $R=0$ den Zusammenhang

$$
|k|=\sqrt{s_{3}^{2}+\left(\frac{(4 n+1) s_{2}}{\left(8 n^{2}+4 n+1\right)}\right)^{2}} .
$$


Um Vergleiche zwischen MDFC und (E)TDAS anstellen zu können, besitzen alle drei Rückkopplungen die Gesamtverstärkung $k$. Für eine erfolgreiche Regelung auf einen Fixpunkt ist zudem immer eine Mindestverstärkung $k^{2}>s_{3}^{2}=d^{2}\left(4 \omega_{c}^{2}-1\right) / 4$ notwendig, um die Destabilisierung $d$ zu kompensieren und reelle Verzögerungszeiten zu gewährleisten. Da die Nenner der Gl. (2.56) und (2.67) gleich sind, bestimmen nur die jeweiligen Zähler die geeigneten Verzögerungszeiten $\tau_{1}+\tau_{2}$ bzw. $\tau$. Durch Variation von $n$ ergeben sich damit für den betrachteten MDFC Spezialfall die $\tau_{1}+\tau_{2}$-Intervalle $[0,2 \pi],[4 \pi, 6 \pi], \ldots$ wohingegen für TDAS die Verzögerungszeit $\tau$ in den Intervallen $[0, \pi],[2 \pi, 3 \pi], \ldots$ gewählt sein muss. Da aber TDAS ein expliziter Sonderfall von MDFC für $\tau_{1}=\tau_{2}$ ist, überlappen sich die Stabilitätsintervalle beider Regelungen, so dass für MDFC die resultierenden Intervalle $[0,3 \pi],[4 \pi, 7 \pi], \ldots$ eine Vergrößerung der Stabilitätsgebiete im Vergleich zu TDAS bedeuten. Durch den gemeinsamen Nenner sind diese Intervalle jeweils bis auf denselben Faktor exakt.

Für ETDAS führt wegen der mit TDAS identischen kritischen Frequenzen $\omega_{s}$ ein anderer Mechanismus zu einer Vergrößerung der Stabilitätsgebiete. Anhand von Gl. (2.65) ist erkennbar, dass durch den Parameter $0<R<1$ für ungerade Vielfache von $\pi$ die Verstärkung $k$ zu einer effektiven Verstärkung $k_{r}$ verringert wird. Wegen der Gleichheit der Zähler der Gl. (2.67), (2.66) und einem verringerten Nenner in Gl. (2.66) ist der Bereich für geeignete Verzögerungszeiten zur Fixpunktstabilisierung für ETDAS vergrößert im Vergleich zu TDAS. Die aus diesen Überlegungen resultierenden Ergebnisse für die Stabilitätsgebiete der einzelnen betrachteten Regelungen sind in Abb. 2.8 dargestellt und kennzeichnen MDFC als die Methode mit den größten Stabilitätsgebieten.

Bei den in Abb. 2.8 schwarz markierten Parameterkombinationen handelt es sich allerdings nur um Ausgangspunkte weiterer Auswahlkriterien für eine erfolgreiche Fixpunktstabilisierung, da in vielen Fällen die Vorfaktoren nicht symmetrisch gewählt werden, d. h. $k_{m a} \neq k_{m b}$. Das betrachtete Nyquist-Diagramm lässt keine Aussage über die Güte der Fixpunktstabilisierung zu. Insofern führen nicht alle in Abb. 2.8 möglichen Parameterkombinationen auch zu gleich guten Stabilitätswerten für den betrachteten Fixpunkt. Um eine Bewertung der Stabilität vornehmen zu können, müsste eine weitere Analyse der Eigenwerte der charakteristischen Gleichung (2.25) erfolgen. Dieses Vorgehen gestaltet sich beim Auffinden optimaler Parameter für MDFC wegen der Transzendenz von Gl. (2.25) allerdings sehr schwierig und soll an dieser Stelle nicht weiter verfolgt werden ${ }^{10}$. Stattdessen kann Abb. 2.7 verwendet werden, um weitere Auswahlkriterien für die Parameter $\tau_{m}$, $k_{m a}$ und $k_{m b}$ zu gewinnen und somit den MDFC-Parameterraum für allgemeine Systeme $\mathbf{f}(\mathbf{x}, \mathbf{u})$ einzuschränken. Ausgangspunkt dieser weiteren Analysen ist die Linearisierung

$$
\begin{aligned}
\dot{\mathbf{x}} & =D \mathbf{f} \cdot \mathbf{x}+\mathbf{b} u \\
y & =\mathbf{c}^{t r} \cdot \mathbf{x}
\end{aligned}
$$

\footnotetext{
${ }^{10}$ Eine Vorgabe von maximalen Stabilitätswerten und anschließende Berechnung der dafür notwendigen Parameter mit Hilfe der charakteristischen Gleichung ist nicht möglich, da zum einen die Maximalwerte der Stabilität bei einem (experimentellen) System a priori nicht bekannt sind und zum anderen die Regelungsparameter durch die Transparenz der Gl. (2.25) nicht analytisch exakt bestimmbar sind.
} 
der Gl.(2.9). Df bezeichnet in diesem Zusammenhang die Jacobi-Matrix des freilaufenden Systems. Betrachtet wird der Fall einer Regelung $u$, die nur eine Systemvariable verwendet und mit Hilfe des Vektors $\mathbf{b}$ auch wieder auf diese zurückkoppelt. Detektiert wird diese Systemvariable durch die Messgröße $y$, die den Systemzustand anhand des geeigneten Auswahlvektors c misst. Laplace-Transformation der Gleichungen (2.70) führt auf

$$
\begin{aligned}
(\lambda I-D \mathbf{f}) \cdot \overline{\mathbf{x}} & =\mathbf{b} \bar{u} \\
\bar{y} & =\mathbf{c}^{t r} \cdot \overline{\mathbf{x}}
\end{aligned}
$$

mit der Einheitsmatrix $I$. Die Vereinigung dieser beiden Bedingungen ergibt die Übertragungsfunktion

$$
T(\lambda)=\frac{\bar{y}(\lambda)}{\bar{u}(\lambda)}=\mathbf{c}^{t r} \cdot(\lambda I-D \mathbf{f})^{-1} \cdot \mathbf{b},
$$

die den Eingang $\bar{u}(\lambda)$ linear mit dem Ausgang $\bar{y}(\lambda)$ des Systems verknüpft. Unter Verwendung der Adjunkten ${ }^{11}$ verändert sich Gl. (2.72) zu

$$
T(\lambda)=\frac{\mathbf{c}^{t r} \cdot \operatorname{Ad}(\lambda I-D \mathbf{f}) \cdot \mathbf{b}}{\operatorname{det}(\lambda I-D \mathbf{f})}
$$

mit dem charakteristischen Polynom des freilaufenden Systems im Nenner von Gl. (2.73). Anregung dieses Systems mit einem $\delta$-Puls ergibt im Fourierraum eine Multiplikation mit Eins, d. h. der Ausgang lautet $\bar{y}(\lambda)=T(\lambda) \cdot \bar{u}_{\delta}=T(\lambda)$ mit $T(\lambda)$ gemäß Gl. (2.73). Dieses Ergebnis stellt wiederum unter Verwendung von $\lambda=i \omega, \omega=2 \pi f$ das Fourierspektrum des freilaufenden Systems dar. Um nun optimale Rückkopplungsparameter zu ermitteln, muss gemäß Abb. 2.7 der Betrag der MDFC-Übertragungsfunktion (2.28) für einen möglichst breiten Frequenzbereich an den der Gl. (2.73) angepasst werden. Zu diesem Zweck empfiehlt es sich, das MDFC Signal

$$
\mathbf{u}(t)=k_{0}+\sum_{m=1}^{M} k_{m a} \mathbf{g}_{m}\left(\mathbf{x}\left(t-\tau_{m}\right)\right)-k_{m b} \mathbf{g}_{m}(\mathbf{x}(t))
$$

durch das modifizierte Signal

$$
\mathbf{u}(t)=k\left[\tilde{k}_{0}+\sum_{m=1}^{M} \tilde{k}_{m a} \mathbf{g}_{m}\left(\mathbf{x}\left(t-\tau_{m}\right)\right)-\tilde{k}_{m b} \mathbf{g}_{m}(\mathbf{x}(t))\right]=k \mathbf{u}_{m t}
$$

zu ersetzen. Auf den ersten Blick wirkt die Einführung des zusätzlichen Parameters $k$ und die dadurch notwendige Reduktion der Verstärkungen zu $\tilde{k}_{0}=k_{0} / k$ sowie $\tilde{k}_{j a}=k_{j a} / k$

\footnotetext{
${ }^{11} \mathrm{Als}$ Adjunkte $\operatorname{Ad}(A)$ der Matrix $A$ wird die transponierte Matrix der Cofaktoren, d. h. der vorzeichenbehafteten Minoren bezeichnet. Die Minoren $M_{j i}$ sind die Werte der Unterdeterminanten der transponierten Matrix $A$, die durch Streichen der $j$-ten Zeile und der $i$-ten Spalte entstehen. Ist $\operatorname{det}(A) \neq 0$, gilt $A^{-1}=\operatorname{Ad}(A) / \operatorname{det}(A)$.
} 

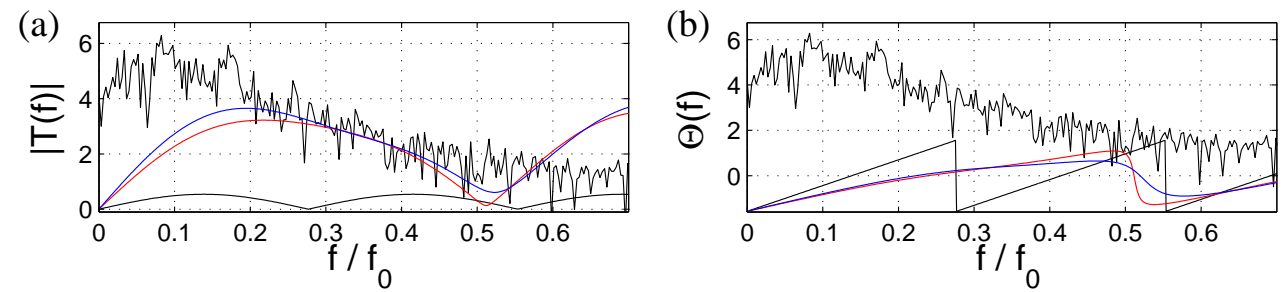

\begin{abstract}
Abb. 2.9: (a) Sukzessive Anpassung der MDFC-Übertragungsfunktion (2.75) an das Spektrum des Colpitts-Oszillators (2.26a). Parameterwerte: $k_{a}=k_{1 b}=0.25$, $\tau_{1} / T=3.614$ (schwarze Linie), $k_{a}=k_{1 b}=0.25, k_{2 a}=k_{2 b}=1.58, \tau_{1} / T=3.614$, $\tau_{2} / T=2.0$ (rote Linie) sowie $k_{a}=k_{1 b}=0.25, k_{2 a}=k_{2 b}=1.58, k_{3 a}=k_{3 b}=0.28$, $\tau_{1} / T=3.614, \tau_{2} / T=2.0, \tau_{3} / T=3.11$. (b) Zugehörige Phase zu den jeweiligen Parameterwerten.
\end{abstract}

und $\tilde{k}_{j b}=k_{j b} / k$ nachteilig und umständlich. Allerdings hat dieses Vorgehen praktische Vorteile. So kann in einem ersten Schritt versucht werden, die nichtinvasive Übertragungsfunktion der Regelung $\mathbf{u}_{m t}$ an das Spektrum des zu regelnden Systems anzupassen. Dabei ist zu beachten, dass die Steilheit der MDFC-Übertragungsfunktion (Nullstelle bei $f=0$ ) für kleine Frequenzen endlich ist und damit in diesem Bereich nicht optimal an das (breitbandige) Spektrum des zu regelnden Systems angepasst werden kann (Abb. 2.7). Ein Beispiel für dieses sukzessive Vorgehen ist in Abb. 2.9 zu sehen. Ausgangspunkt ist dabei das Aktivieren einer einzigen Verzögerungszeit (schwarze Linie). Durch das Hinzuschalten weiterer geeignet verstärkter Verzögerungszeiten kann der Verlauf des Fourierspektrums des Colpitts-Oszillators immer feiner approximiert werden. Sind die Koeffizienten $\tilde{k}_{j a}, \tilde{k}_{j a}$ und $\tilde{k}_{0}$ festgelegt,muss anschließend $k$ variiert werden, um die maximale Stabilität zu erreichen. Häufig wird dieses Verfahren zum Einstellen der Parameter allerdings durch das Vorhandensein einer Totzeit erschwert.

\title{
2.8.2 Rückkopplung mit Totzeit
}

Die Wirkungsweise einer Regelung auf ein dynamisches System unter Einfluss einer Totzeit $\delta>0$ war bereits Gegenstand vielfältiger Untersuchungen. Kürzlich wurde dieser Aspekt auch auf chaotische Systeme unter dem Gesichtspunkt der Fixpunkt- bzw. Orbitstabilisierung ausgedehnt $[58,59]$. Totzeiten können vielfältige Ursachen haben. Zum einen können sie bereits systemimmanent sein, wie das beispielsweise in einem Lasersystem der Fall ist, wo eine Veränderung des Pumpstromes nicht instantan zu einer Veränderung der Ausgangsintensität führt. Desweiteren führt natürlich die Regelung selbst, d.h. die Aufnahme und Verarbeitung von Signalen bis zur Bereitstellung des Regelsignals zu einer Totzeit, in der dem zu kontrollierenden System nicht das optimale Regelungssignal zugeführt werden kann. Ist die Totzeit klein im Vergleich zur Zeitskala der Schwingungen des zu regelnden Systems, so stellen sich keine Schwierigkeiten ein, im Gegensatz zu schnel- 
len dynamischen Systemen, wo selbst kleine Totzeiten schon zu merklichen Problemen führen. In den folgenden Abschnitten werden Größen unter Totzeiteinfluss immer durch einen Index .tot gekennzeichnet sein. Wird vergleichbar zu Abschnitt 2.8.1 der Spezialfall des harmonischen Oszillators (2.37) betrachtet, so führt die Annahme einer Totzeit $\delta$ für Gl. (2.36) zu dem modifizierten Regelsignal

$$
u(t)=\sum_{m=1}^{M} k_{m a} x\left(t-\left(\tau_{m}+\delta\right)\right)-k_{m b} x(t-\delta)
$$

führt. Laplace-Transformation liefert wiederum den generellen funktionellen Zusammenhang

$$
u_{R, \text { tot }}=e^{-\lambda \delta} u_{R}
$$

zwischen totzeitbeeinflusster und -unbeeinflusster Größe, wobei das bereits transformierte MDFC Signal

$$
u_{R}=\sum_{m=0}^{M} k_{m a} e^{-\lambda \tau_{m}}
$$

mit $\tau_{0}=0, k_{0 a}=-\sum_{m=1}^{M} k_{m a}$ zum Einsatz kommt. Dadurch ändert sich die NyquistFunktion (2.41) in

$$
N_{\text {tot }}(i \omega)=-H(i \omega) C_{\text {tot }}(i \omega)=-e^{-i \omega \delta} \frac{\sum_{m=0}^{M} k_{m a} e^{-i \omega \tau_{m}}}{\omega_{0}^{2}-\omega^{2}+i d \omega},
$$

wodurch sich der Phasenwinkel $\Theta_{\text {tot }}$ unter Verwendung der Abkürzungen aus Gl. (2.43) und (2.44) durch

$$
\Theta_{\text {tot }}=\arctan \left(\frac{\cos (\omega \delta) b+\sin (\omega \delta) a}{-\cos (\omega \delta) a+\sin (\omega \delta) b}\right)=\arctan \left(\frac{1+\frac{1}{m} \tan (\omega \delta)}{\tan (\omega \delta)-\frac{1}{m}}\right)
$$

mit $m=b / a$ beschreiben lässt. Nach Anwendung des Additionstheorems [31]

$$
\tan (\alpha \pm \beta)=\frac{\tan (\alpha) \mp \tan (\beta)}{1 \pm \tan (\alpha) \tan (\beta)}
$$

kann Gl. (2.80) in den Ausdruck

$$
\Theta_{\text {tot }}=\arctan \left(\frac{1}{\tan (\omega \delta-\gamma)}\right)=\arctan (\cot (\omega \delta-\gamma))
$$

mit $\gamma=\arctan (b / a)$ überführt werden, was ebenfalls unter Beachtung von [31]

$$
\arctan (\alpha)=\frac{\pi}{2}-\operatorname{arccot}(\alpha)
$$


für den Phasenwinkel in Gegenwart einer Totzeit zu

$$
\Theta_{\mathrm{tot}}=l \pi-\omega \delta+\arctan \left(\frac{b}{a}\right), \quad l \in\{0,1\}
$$

führt. An dieser Stelle wurde zudem noch der Zusammenhang [31]

$$
\arctan (\alpha)+\arctan \left(\frac{1}{\alpha}\right)= \begin{cases}\frac{\pi}{2} & \alpha>0 \\ -\frac{\pi}{2} & \alpha<0\end{cases}
$$

ausgenutzt. Das Ergebnis aus Gl. (2.84) ist nicht sonderlich überraschend und zeigt nur, dass sich der Phasenwinkel des Gesamtsystems aus dem des dynamischen Systems und dem durch die Totzeit der (Regel-)Strecke zusammensetzt.

Im Falle einer verschwindenden Totzeit $\delta=0, l=0$ ergibt sich der schon in Gl. (2.42) dargestellte Zusammenhang, so dass die folgenden Resultate lediglich eine Erweiterung zu den bereits präsentierten darstellen, die auch weiterhin ihre Gültigkeit behalten. Nach den Ausführungen in Abschnitt 2.8.1 bezüglich fehlender Totzeit $\delta$ ist bekannt, dass die kritischen Frequenzen $\omega_{s}$ durch die Bedingung

$$
\begin{array}{ll}
-\pi+l \pi+\omega \delta+\arctan \left(\frac{b}{a}\right)=-(2 n+1) \pi, & 0<\omega<\omega_{r}, \\
-2 \pi+l \pi+\omega \delta+\arctan \left(\frac{b}{a}\right)=-(2 n+1) \pi, & \omega>\omega_{r}
\end{array}
$$

mit $\omega_{r}=\sqrt{\omega_{0}^{2}-d^{2} / 2}$ gegeben sind, d.h. es ist die Gleichung

$$
\frac{b}{a}=\tan \left((2 n+l) \pi-\omega_{s} \delta\right)
$$

für die kritischen Frequenzen $\omega_{s}$ zu erfüllen. Für eine Regelung wird es generell schwierig, wenn (plötzlich) Vorzeichenwechsel auftreten, die die Regelung von einer Gegenkopplung in eine Mitkopplung überführen. Die (abrupten) Vorzeichenwechsel des Ausdrucks $\tan \left((2 n+l) \pi-\omega_{s} \delta\right)$ sind durch $(2 n+l) \pi-\omega_{s} \delta=(2 m+1) \pi / 2$ gegeben. Entspricht die Totzeit

$$
\delta=\frac{s \pi}{2 \omega_{s}}
$$

so ist keine Kontrolle der Dynamik möglich, wobei $s$ eine ungerade Zahl $\in \mathbb{N}$ ist. In diesem Fall sind die Phasen der Mit- und die der Gegenkopplung gleich lang, d. h. effektiv ist keine Regelungswirkung festzustellen. Die Perioden der Mit- bzw. Gegenkopplung entsprechen gerade der halben Periode des betrachteten harmonischen Oszillators (2.37). Wird die Regelung durch die Totzeit $\delta$ in den Bereich der Mitkopplung geführt, kann diese durch einen Vorzeichenwechsel der Verstärkungsfaktoren wieder in eine Gegenkopplung umgewandelt werden. 
Gl. (2.86) kann weiterhin mit $r \in \mathbb{Z} \mathrm{zu}$

$$
\arctan \left(\frac{b}{a}\right)=r \pi+\omega_{s} \delta
$$

bzw.

$$
\frac{b}{a}=\tan \left(r \pi+\omega_{s} \delta\right)=\frac{\tan (r \pi)+\tan \left(\omega_{s} \delta\right)}{1+\tan (r \pi) \tan \left(\omega_{s} \delta\right)}=\tan \left(\omega_{s} \delta\right)
$$

vereinfacht werden. Entsprechend der Gl. (2.49), (2.53) ergibt sich für MDFC mit zwei Verzögerungszeiten und gleich gewählten Vorfaktoren $k_{1}=k_{2}=k / 2$ der Ausdruck

$$
\tan \left(\omega_{s} \frac{\tau_{1}+\tau_{2}}{2}\right)=\tan \left(\omega_{s} \delta\right)
$$

für (E)TDAS unter Verwendung der analogen Gleichungen das Ergebnis

$$
\tan \left(\omega_{s} \tau\right)=\tan \left(\omega_{s} \delta\right)
$$

Demnach muss versucht werden, mit den Verzögerungszeiten $\tau_{1}, \tau_{2}$ bzw. $\tau$ die Totzeit $\delta$ auszugleichen. Das ist allerdings nicht für alle Totzeiten $\delta>0$ möglich, da sowohl die Verzögerungszeiten als auch die damit korrespondierenden Verstärkungen entsprechend den vorherigen Untersuchungen bestimmten Grenzwerten unterliegen. Das Auftreten einer Totzeit schränkt demnach die Stabilitätsgebiete aller Regelungen ein, da die vorher ermittelten Stabilitätskriterien auch bei Präsenz einer Totzeit gewahrt werden müssen. Nach dem im Abschnitt 2.8.1 beschriebenen Vorgehen berechnen sich hierbei die kritischen Frequenzen $\omega_{s}$ für MDFC mit zwei Verzögerungszeiten und gleichen Vorfaktoren $k / 2$ sowie für (E)TDAS zu

\begin{tabular}{c|c|c|c|c} 
& $\operatorname{TDAS}\left(\omega_{s}\right)$ & $\operatorname{ETDAS}\left(\omega_{s}\right)$ & $\operatorname{NTDAS}\left(\omega_{s}\right)$ & $\operatorname{MDFC}\left(\omega_{s}\right)$ \\
\hline $0<\omega<\omega_{r}$ & $\frac{(2 n+l) \pi}{\tau+\delta}$ & $\frac{(2 n+l) \pi}{\tau+\delta}$ & $\frac{(2 n+l) \pi}{\tau+\delta}$ & $\frac{2(2 n+l) \pi}{\tau_{1}+\tau_{2}+2 \delta}$ \\
$\omega>\omega_{r}$ & $\frac{(2 n+1+l) \pi}{\tau+\delta}$ & $\frac{(2 n+1+l) \pi}{\tau+\delta}$ & $\frac{(2 n+1+l) \pi}{\tau+\delta}$ & $\frac{2(2 n+1+l) \pi}{\tau_{1}+\tau_{2}+2 \delta}$
\end{tabular}

was zu den geeigneten Verzögerungszeiten

$$
\frac{4 n \pi}{\sqrt{s_{2}-\sqrt{k^{2}-s_{3}^{2}}}}-2 \delta<\tau_{1}+\tau_{2}<\frac{2(2 n+1) \pi}{\sqrt{s_{2}+\sqrt{k^{2}-s_{3}^{2}}}}-2 \delta
$$

für MDFC bzw. 

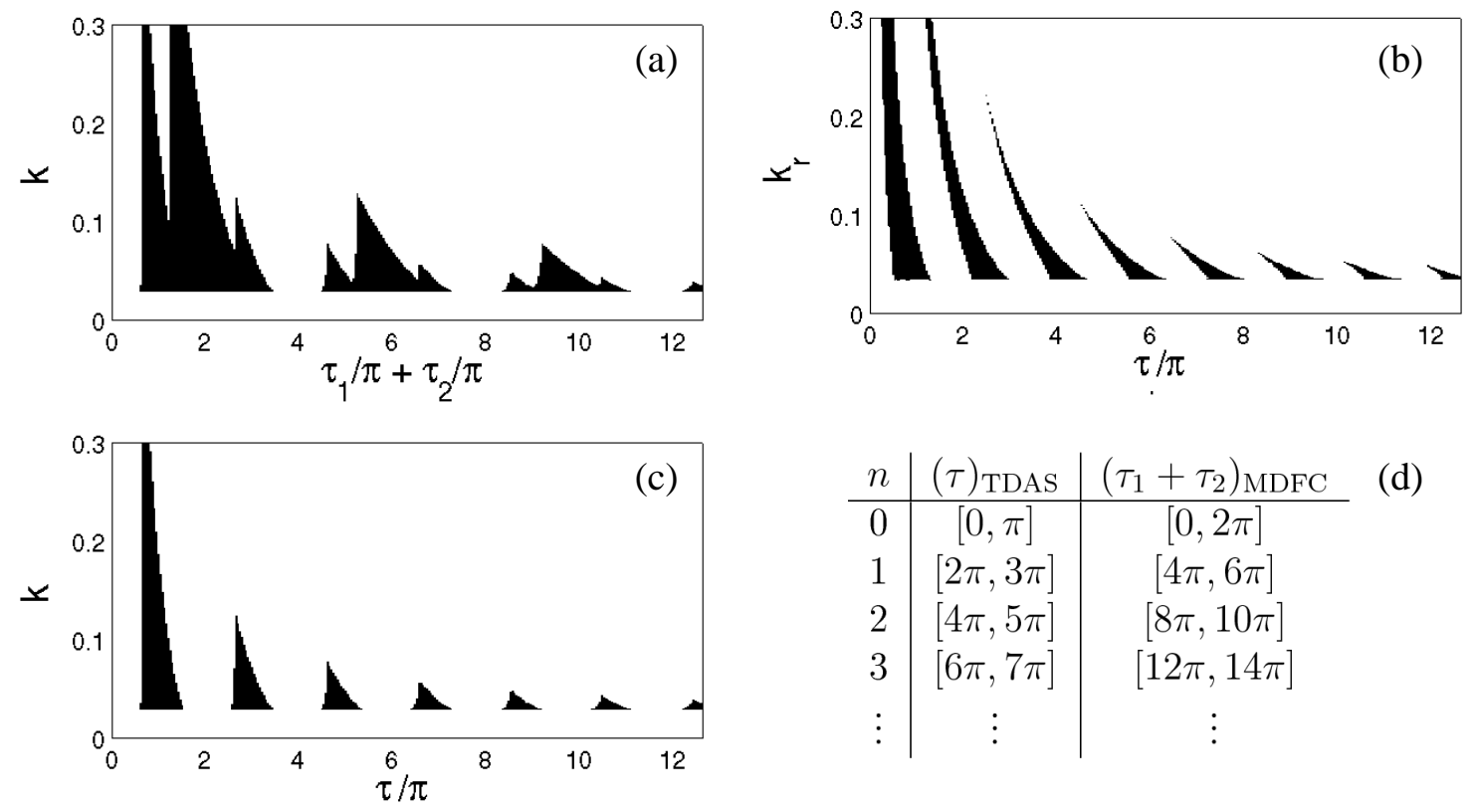

\begin{tabular}{c|c|c}
$n$ & $(\tau)_{\mathrm{TDAS}}$ & $\left(\tau_{1}+\tau_{2}\right)_{\mathrm{MDFC}}$ \\
\hline 0 & {$[0, \pi]$} & {$[0,2 \pi]$} \\
1 & {$[2 \pi, 3 \pi]$} & {$[4 \pi, 6 \pi]$} \\
2 & {$[4 \pi, 5 \pi]$} & {$[8 \pi, 10 \pi]$} \\
3 & {$[6 \pi, 7 \pi]$} & {$[12 \pi, 14 \pi]$} \\
$\vdots$ & $\vdots$ & $\vdots$
\end{tabular}

(d)

Abb. 2.10: Stabilitätsgebiete (schwarz markiert) des harmonischen Oszillators (2.35) in Abhängigkeit von der Verstärkung $k$ und der (den) Verzögerungszeit(en) $\tau$ bzw. $\tau_{1}+\tau_{2}$ bei einer Totzeit von $\delta=1.2$ für (a) MDFC mit zwei Verzögerungszeiten und gleichen Verstärkungen $k / 2$ sowie $\tau$ für (b) ETDAS mit $R=0.7$ und (c) TDAS. Die Verstärkung in (b),(c) ist zu $k$ gewählt, die Parameter aus Gl. (2.35) sind auf $\omega_{0}^{2}=1$ und $d=-0.1$ fixiert. (d) TDAS und MDFC im Vergleich: Bedingungen für die Stabilisierung des Fixpunktes für den harmonischen Oszillator in Abhängigkeit von der Verzögerung.

$$
\frac{2 n \pi}{\sqrt{s_{2}-\sqrt{s_{4}^{2}-s_{3}^{2}}}}-\delta<\tau<\frac{(2 n+1) \pi}{\sqrt{s_{2}+\sqrt{s_{4}^{2}-s_{3}^{2}}}}-\delta,
$$

für (E)TDAS führt. Im Fall von ETDAS ist $s_{4}$ entsprechend Gl. (2.65) zu verwenden. Für TDAS ist $s_{4}=k$ erfüllt, wobei $k$ die Verstärkung bezeichnet. Entsprechendes Vorgehen wie in Abschnitt 2.8.1 liefert bei Betrachtung des Betrages der Amplitude der NyquistFunktion $N\left(i \omega_{s}\right)$ (2.41) die in Abb. 2.10 dargestellten Stabilitätsgebiete. Deren größere Ausdehnung für MDFC im Vergleich zu (E)TDAS bietet auch in Gegenwart einer Totzeit $\delta>0$ mehr Möglichkeiten, die zusätzlichen Bedingungen (2.91), (2.92) zu erfüllen. So sind in Abb. 2.10 generell kleinere Stabilitätsgebiete zu erkennen, von denen MDFC die größten garantiert.

In Gegenwart einer systemimmanenten Totzeit verschieben sich die Eigenwerte zu positiveren Realteilen, der betrachtete Fixpunkt wird instabiler. Kritisch für eine zeitverzögerte Regelung ist in diesem Zusammenhang der Einsatz von sehr langen Verzögerungszeiten, 
was dazu führt, dass Eigenwerte in der komplexen Ebene sehr schnell miteinander kollidieren und nicht in den stabilen Bereich verschoben werden können. Von daher wäre bei Einsatz von mehreren $\tau_{m}$ eine Wahl von mehreren entsprechend kurzen Verzögerungszeiten zu empfehlen. Das Vorzeichen der einzelnen Vorfaktoren muss je nach Länge der Totzeit ebenfalls angepasst werden, damit das Regelsignal effektiv eine Gegen- und nicht eine Mitkopplung bewirkt.

\subsection{Koexistierende Fixpunkte: Anfangswertproblem}

Neben der Stabilität an sich ist natürlich bei Systemen mit mehreren koexistierenden Fixpunkten die Frage interessant, zu welchem der Fixpunkte die Systemdynamik bei Aktivierung der Regelung konvergiert und ob mit Hilfe der Regelparameter das System hinsichtlich einer bestimmten Zieldynamik beeinflusst werden kann. Für diesen Zweck wird ein System betrachtet, das über genau zwei symmetrisch im Phasenraum plazierte Strudelpunkte verfügt, die Paare instabiler, komplex konjugierter Eigenwerte besitzen. Ein geeignetes dynamisches System, das genau diesen Anforderungen entspricht, ist durch die Gleichungen

$$
\begin{aligned}
& \dot{x}=y z+u \\
& \dot{y}=x-y \\
& \dot{z}=1-x y
\end{aligned}
$$

(a)

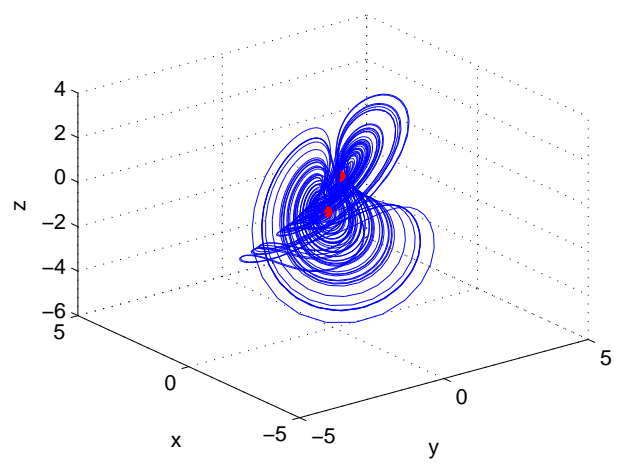

(b)
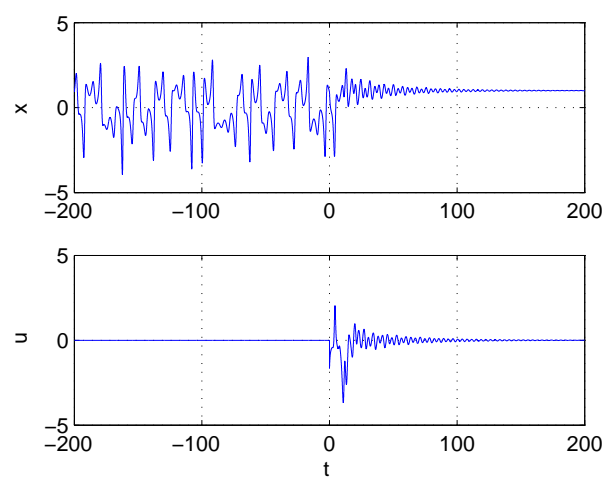

Abb. 2.11: (a) Chaotischer Attraktor des ungeregelten $(\mathbf{u}=0)$ Systems (2.95). (b) System (2.95) kontrolliert durch das MDFC-Signal (2.96) basierend auf zwei Verzögerungszeiten $\tau_{1}=10.2, \tau_{2}=7.0$ und $k_{1}=0.3, k_{2}=0.9$. Die Regelung wirkt auf die $x$-Variable des Systems und wird zur Zeit $t=0$ aktiviert. Für diese Parameterkombination wird der obere Fixpunkt stabilisiert, das Regelsignal verschwindet nach Erreichen der Zieldynamik. 
gegeben [143]. Gl. (2.95) generiert den in Abb. 2.11a dargestellten chaotischen Attraktor, der über die beiden Fixpunkte $\mathbf{x}_{0 a}=\left(x_{0 a}, y_{0 a}, z_{0 a}\right)=(1,1,0)$ und $\mathbf{x}_{0 b}=\left(x_{0 b}, y_{0 b}, z_{0 b}\right)=$ $(-1,-1,0)$ verfügt. Zur Stabilisierung dieser beiden Fixpunkte kommt das von der $x$ Komponente abhängige und auch wieder auf diese wirkende MDFC-Signal $\mathbf{u}=(u(t), 0,0)^{t r}$ mit

$$
u(t)=\sum_{m=1}^{M} k_{m}\left(x\left(t-\tau_{m}\right)-x(t)\right)
$$

und maximal $M$ verschiedenen Verzögerungszeiten $\tau_{m}$ sowie symmetrischen Vorfaktoren $k_{m a}=k_{m b}=k_{m}$ zum Einsatz. Unter Beachtung der vorher vorgestellten linearen Stabilitätsanalyse ergibt sich für den betrachteten Fixpunkt $\mathbf{x}_{0 a}$ die charakteristische Gleichung

$$
\operatorname{det}(\Delta(\lambda))=\left(\lambda-u_{R}\right)(\lambda+1) \lambda+x_{0 a} y_{0 a}+y_{0 a}^{2}(\lambda+1)-\lambda z_{0 a}=0,
$$

deren Analyse für zwei und mehr Verzögerungszeiten auf ähnliche Stabilitätsdiagramme führt wie für das Colpitts-System aus Gl. (2.26a) [7].

\begin{abstract}
Abb. 2.12: Horizontaler Schnitt durch einen $\tau_{1}-\tau_{2}$-Stabilitätsplot mit der Stabilitätsfunktion $\max (\operatorname{Re}(\lambda))$ und $\tau_{1}=3.0$ sowie steigenden Verstärkungsfaktoren, $k_{1}=$ $0.5, k_{2} \in[0.1,1.0]$. Dargestellt ist der Eigenwert mit dem größten Realteil $\lambda$. Eine Stabilisierung ist möglich für $k_{2}>0.3$, was durch die blaue Farbe kenntlich gemacht wurde, eine Verstärkungserhöhung verschiebt den Eigenwert mit dem größten Realteil hauptsächlich zu stabileren (negativeren) Werten.
\end{abstract}

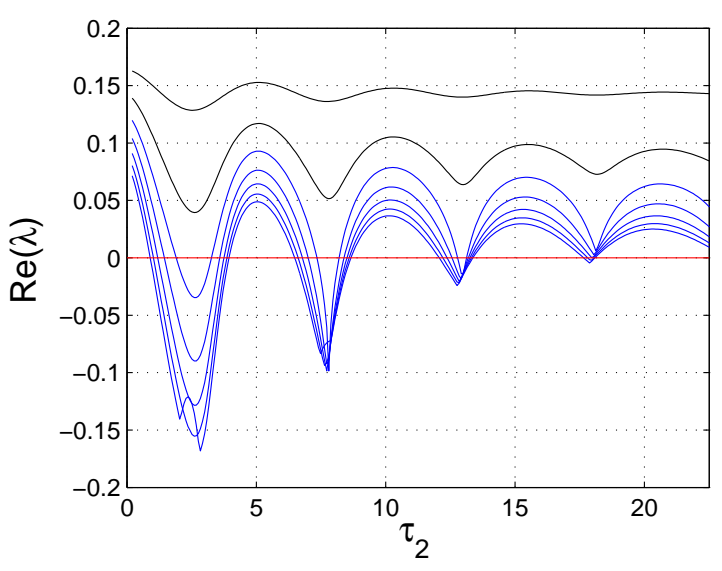

Nach Betrachtung aller bisher gelieferten Ergebnisse ist klar, dass MDFC Stabilitätsgebiete stärker erweitert als alle anderen üblichen Verzögerungsmethoden. Durch die lineare Stabilitätsanalyse kann allerdings immer nur ein Fixpunkt betrachtet werden. Dabei stellt sich heraus, dass die beiden Fixpunkte der Gl. (2.95) über identische Stabilitätsdiagramme verfügen. ${ }^{12}$ Letztendlich entscheiden die Anfangsbedingungen und Bassins ${ }^{13}$ darüber, zu welchem Fixpunkt die Dynamik konvergiert, sofern die koexistierenden Fixpunkte alle mit der betrachteten Regelung stabilisiert werden können. Abb. 2.13 zeigt (asympto-

\footnotetext{
${ }^{12}$ Die Symmetrie des dynamischen Flusses vererbt sich in diesem Fall durch die charakteristische Matrix auch auf die charakteristische Gleichung. Zur Verifizierung dieses Sachverhaltes müssen lediglich die beiden Fixpunkte $\mathbf{x}_{0 a}=(1,1,0)$ und $\mathbf{x}_{0 b}=(-1,-1,0)$ in Gl. (2.97) eingesetzt werden.

${ }^{13} \mathrm{Zu}$ jedem Attraktor gehört ein Einzugsbereich innerhalb des Phasenraumes, von dem aus die Dynamik immer zum betrachteten Attraktor konvergiert. Verfügt das betrachtete System über mehrere Attraktoren, gibt es koexistierende Bassins.
} 
(a)

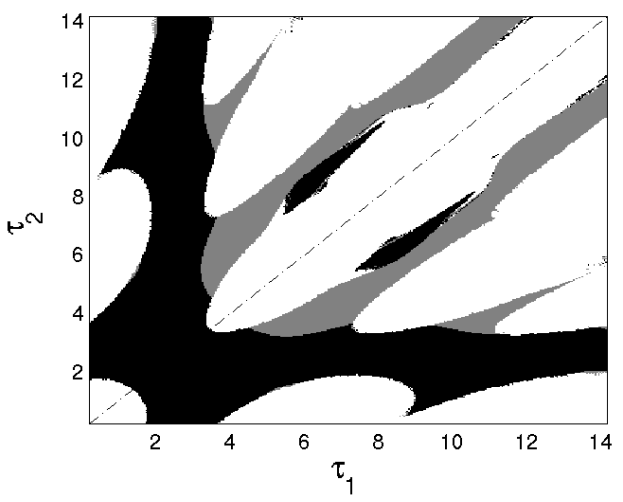

(b)

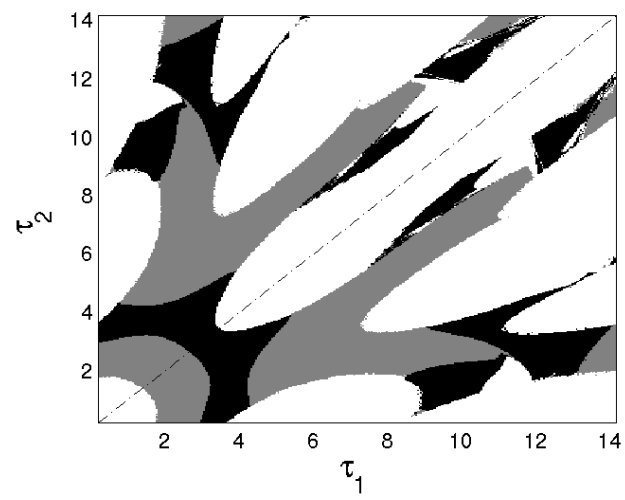

Abb. 2.13: Kombinationen der Verzögerungszeiten $\tau_{1}$ und $\tau_{2}$, für die MDFC mit zwei Verzögerungszeiten $\left(k_{1}=k_{2}=0.5\right)$ und den Anfangsbedingungen $\left(x_{s}, y_{s}, z_{s}\right)$ nicht zur Fixpunktstabilisierung führt, sind weiss dargestellt. Solche, die für diese Anfangswerte die Stabilisierung des oberen Fixpunktes $\mathbf{x}_{0 a}=(1,1,0)$ herbeiführen, sind schwarz markiert, entsprechende Kombinationen für die Stabilisierung des unteren Fixpunktes $\mathbf{x}_{0 b}=(-1,-1,0)$ sind grau gekennzeichnet. Zur Berechnung der Diagramme aus (a) und (b) wurden die Anfangsbedingungen jeweils festgehalten. Anfangswerte der Integration: (a) $\left(x_{s}, y_{s}, z_{s}\right)=(0.1,-0.1,0.1)$, (b) $\left(x_{s}, y_{s}, z_{s}\right)=(-0.3,-0.3,0.1)$.

tische) Stabilitätsdiagramme für zwei verschiedene Konfigurationen von Anfangswerten für das durch MDFC mit zwei Verzögerungszeiten $\left(k_{1}=k_{2}=0.5\right)$ kontrollierte System (2.95). Die Berechnung erfolgte durch nummerische Integration der Differentialgleichungen (2.95). Die Anfangsbedingungen wurden für jede $\tau_{1}, \tau_{2}$-Kombination auf $(0.1,-0.1,0.1)$ (Abb. 2.13a) bzw. (-0.3, -0.3, 0.1) (Abb. 2.13b) fixiert, die Aktivierung der Regelung erfolgte immer zur selben Zeit. Regionen, die nach Integration zum oberen Fixpunkt $\mathbf{x}_{0 a}=(1,1,0)$ führen, sind schwarz gekennzeichnet, solche, die die Dynamik zum unteren Fixpunkt $\mathbf{x}_{0 b}=(-1,-1,0)$ leiten, grau. Weisse Gebiete repräsentieren Parameterkombinationen, die keine Fixpunktstabilisierung ermöglichen. Daran läßt sich erkennen, dass die Dynamik durch Veränderung von $\tau_{1}$ und $\tau_{2}$ direkt zu einem der beiden Fixpunkte gelenkt werden kann.

\subsection{Realisierungen von Zeitverzögerungen}

Verzögerungszeiten können in vielfältiger Art und Weise erzeugt werden, z. B. elektrisch. Die einfachste Variante stellt dabei eine Signalverzögerung entlang einer (BNC-)Leitung aufgrund der endlichen Ausbreitungsgeschwindigkeit des Signals dar. Dieses Vorgehen bietet natürlich für sehr kurze Verzögerungszeiten eine besonders einfache Implemen- 
tierungsmöglichkeit. Die Situation ändert sich allerdings, wenn die geforderten Verzögerungszeiten lang sein sollen. Ein weiteres Problem ergibt sich in Gegenwart von Instationaritäten, wenn die Verzögerungszeiten in kurzen Abständen wesentlich variiert werden müssen. Das ist natürlich mit einer (BNC-)Leitung mit starrer Länge nicht zu erreichen. Abhilfe schafft dann eine Filterkette bestehend aus Allpassgliedern, deren Anzahl von der maximal benötigten Verzögerungszeit abhängt. Dieses Vorgehen ist auch in Gegenwart von System-Instationaritäten praktikabel, da die Variation der effektiven Verzögerung in diesem elektrischen System mit Hilfe einer Widerstandseinstellung vonstatten geht. Es ist allerdings darauf zu achten, dass der Einstellbereich pro Allpassfilter nicht zu groß gewählt wird, um Verzerrungen bei der Signalverzögerung zu vermeiden. In der Natur gibt es noch andere Mechanismen, um Signalverzögerungen zu realisieren, z. B. mit Hilfe von Neuronen. Die eigentliche Verzögerung resultiert in diesem Zusammenhang daraus, dass Neuronen nur innerhalb einer endlichen Zeitspanne feuern, d. h. elektrische Impulse aussenden können. Nach Abgabe dieser sogenannten Bursts benötigen Neuronen eine

Abb. 2.14: MDFC-Rückkopplung (2.99) für das Hindmarsh-Rose-System (2.98) (2 systemeigene Zeitskalen) mit den Parametern $k_{1 a}=k_{1 b}=1.37, k_{2 a}=k_{2 b}=1.61$, $k_{3 a}=k_{3 b}=1.31, \tau_{1}=140.8, \tau_{2}=171.6$ sowie $\tau_{3}=268$.

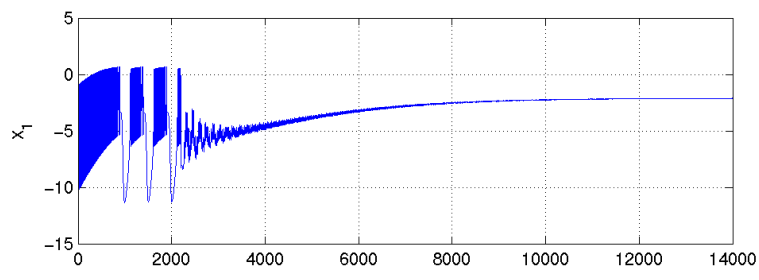

bestimmte Erholzeit, bis das nächste Eingangssignal verarbeitet werden kann. Diese Neuronendynamik kann z. B. mit dem Hindmarsh-Rose-System [57]

$$
\begin{aligned}
& \dot{x}=y+x^{2}(3-x)-z+I \\
& \dot{y}=1-5 x^{2}-y+u(t) \\
& \dot{z}=\epsilon(4(x+h)-z)
\end{aligned}
$$

unter Verwendung der Parametern $\epsilon=0.001, h=1.6$ und $I=3$ simuliert werden. Die dabei auftretenden (chaotischen) Oszillationen spielen sich auf zwei unterschiedlichen Zeitskalen ab. Dabei wird im wesentlichen eine Reihe schneller Bursts auf eine langsamere Grundschwingung aufmoduliert. Abgesehen von der Erzeugung langer Verzögerungszeiten kann das Hindmarsh-Rose-System (2.98) auch unter dem Gesichtspunkt der Chaoskontrolle betrachtet werden. Ist es erforderlich, ein feuerndes Neuron auszuschalten (z. B. zur Schmerzunterdrückung), so kann dieses durch das MDFC Signal

$$
u(t)=\sum_{m=1}^{3} k_{m a} y\left(t-\tau_{m}\right)-k_{m b} y(t)
$$

geschehen. Abb. 2.14 veranschaulicht diesen Sachverhalt für die Regelparameter $k_{1 a}=$ $k_{1 b}=1.37, k_{2 a}=k_{2 b}=1.61, k_{3 a}=k_{3 b}=1.31, \tau_{1}=140.8, \tau_{2}=171.6$ sowie $\tau_{3}=268$ mit 
deren Hife der systemeigene Fixpunkt mit den Koordinaten $\mathbf{x}_{0} \approx(-0.7878,-2.099,3.251)$ stabilisiert wird. Was hierbei zu erkennen ist, ist die Tatsache, dass die Regelung mit diesen gewählten Regelparametern eine sehr lange Zeit benötigt, bis das System wirklich endgültig auf den Fixpunkt relaxiert. Weiterhin ist anzumerken, dass mit Hilfe zweier zeitverzögerter Terme zwar eine sehr deutliche Reduzierung der Bursts herbeigeführt werden kann, der Erfolg beim Einsatz dreier Terme für die Rückkopplung aber entschieden größer ist und im Erreichen des Fixpunktes $\mathbf{x}_{0}$ resultiert.

\section{$2.11 \quad$ Weitere Details}

Der Erfolg von MDFC aus Gl. (2.16) basiert auf der Verwendung von zwei oder mehr aktiven Verzögerungszeiten sowie einer individuellen Gewichtung der einzelnen Rückkopplungsterme. Eine Möglichkeit, zwei Verzögerungszeiten bei nur einer vorhandenen Verzögerungskette anzuwenden, liegt in einer Modulation der Verzögerungszeit $\tau$. So könnte z. B. diese Verzögerungszeit $\tau$ durch ein entsprechend angepasstes rechteck- oder sinusförmiges Signal moduliert werden. Dieses Vorgehen ist zwar für die Stabilisierung bzw. Generierung von periodischen Orbits im TDAS-Fall aus Gl. (2.10) erfolgreich [88], aber weder in der Theorie noch in der Praxis zur Stabilisierung von Fixpunkten geeignet. Es ist sogar eher das Gegenteil der Fall. Durch Modulation der Verzögerungszeit $\tau$ schrumpfen die Stabilitätsgebiete für (E)TDAS und MDFC bezüglich Fixpunktstabilisierung im Vergleich zu starren Verzögerungszeiten.

Weiterhin stellt sich auch kein Erfolg in Experimenten ein, wenn die Vorfaktoren (Gewichte) periodisch moduliert werden. Diese Beobachtung lässt sich bereits bei [137] für TDAS finden. Auch diese Modifikation führt zu einer Verringerung der Stabilität. Andererseits ist eine langsame und geeignete Anpassung der MDFC-Vorfaktoren $k_{m a}, k_{m b}$ bei Auftreten von Instationaritäten in der Praxis von Interesse.

In [72] wurde zur Stabilisierung von periodischen Orbits neben der Modulation einzelner Komponenten des Regelsignals mit den Floquet-Eigenfunktionen eine Rückkopplung via Diagonalkontrolle [20] vorgeschlagen, die auch für die Stabilisierung von Fixpunkten geeignet erscheint. Dabei wird jede Systemvariable in Form analog aufgebauter Regelgleichungen wieder auf sich selbst zurückgekoppelt, wodurch die jeweiligen (gleichen) Regeleinträge nur auf der Diagonalen der charakteristischen Matrix auftreten. Auf diese Weise wird die charakteristische Matrix entkoppelt und analytisch exakte Aussagen sind im Falle einer einzigen Verzögerungszeit $\tau$ (TDAS) z. B. für den harmonischen Oszillator mit Hilfe von Lambert-Funktionen möglich [58]. Dieses Vorgehen hat zwar bei der nummerischen wie auch theoretischen Untersuchung von Systemen Vorteile, gestaltet sich aber experimentell in vielen Fällen schwierig, da nicht immer alle Variablen auch gleichzeitig Observable und damit teilweise auch nicht direkt beeinflussbar sind. 


\section{Kapitel 3}

\section{Von MDFC zu NFF}

\section{1 Übergang vom Zeit- in den Frequenzbereich}

Die Betrachtung der Übertragungsfunktion des im Zeitbereich wirkenden MDFC (2.16) zeigt, dass erfolgreiche Fixpunktkontrolle mit einer intelligenten Verteilung von Kerben im Frequenzraum verknüpft ist. Um ähnliche Ergebnisse ohne Zeitverzögerung zu erzielen, kann die MDFC-Übertragungsfunktion alternativ lokal durch lineare Kerbfilter approximiert werden. Um diesen Gesichtspunkt weiter zu beleuchten, wird wiederum der Colpitts-Oszillator (2.26a) betrachtet, der für das freilaufende System $(\mathbf{u}=0)$ chaotische Dynamik offenbart. Abb. 3.1a zeigt das zugehörige breitbandige Frequenzspektrum des Colpitts-Systems, das über einige dominante Peaks verfügt, die im chaotischen Attraktor eingebetteten UPOs entsprechen. Die resultierende chaotische Dynamik (Abb. 3.1b) kann unter Anwendung des TDAS-Kontrollsignals [117]

$$
u(t)=k\left[U_{C_{1}}(t)-U_{C_{1}}(t-\tau)\right]
$$

auf den in Abb. 3.1d gezeigten Periode-1-Orbit stabilisiert werden. Die Regelung wirkt zu diesem Zweck auf die erste Komponente von Gl. (2.26a) und verwendet die Parameter $k=-0.72$ sowie $\tau=6.66$. Der Betrag der entsprechenden Übertragungsfunktion $T(f)=\exp (-i 2 \pi f \tau)-1$ mit $\tau=6.66$ und $k=1$ ist in Abb. 3.1c dargestellt. Da die Verzögerungszeit $\tau$ gerade der Periode des zu stabilisierenden UPOs entspricht, fallen alle Kerben der Übertragungsfunktion mit den Harmonischen dieses UPOs zusammen, der somit nicht verzerrt wird. Folglich werden alle anderen Frequenzen, die nicht zu diesem UPO gehören, eliminiert. Von daher wird dieser UPO nicht verändert, es handelt sich um eine nichtinvasive Kontrolle. Fast dasselbe Resultat kann aber auch erreicht werden, indem die Verzögerungsregelung in (3.1) durch ein (lineares) Kerbfilter (notch filter feedback, NFF) ersetzt wird [8], das die höherharmonischen Kerben der TDAS-Kontrolle vernachlässigt. Ein Beispiel für ein solches Filter ist durch das bekannte Wien-Robinson-Filter [148] gegeben, dessen Übertragungsfunktion in Abb. 3.1e zu sehen ist. In den durchgeführten 

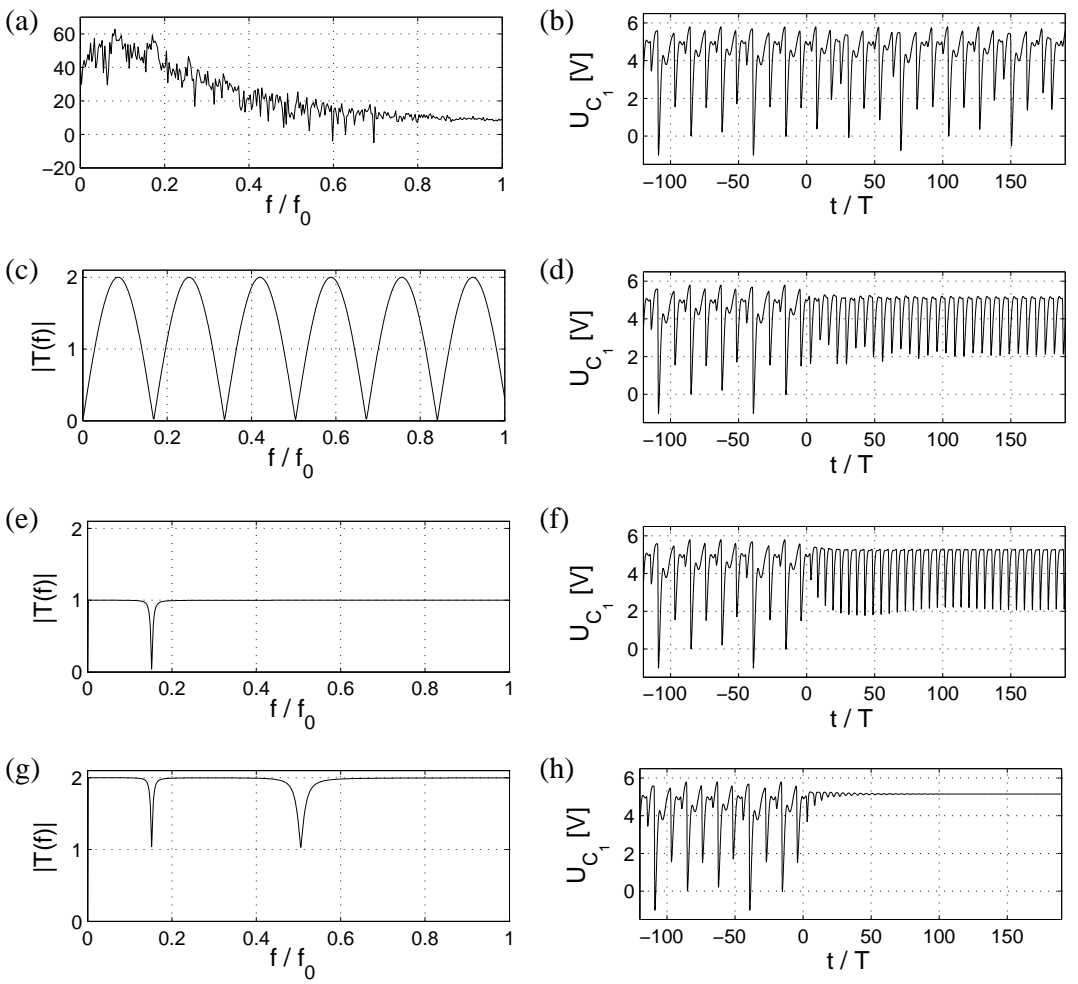

Abb. 3.1: Kontrolle des chaotischen Colpitts-Oszillators (2.26a) durch verschiedene Kontrollmethoden. Spektrum (a) und chaotische Zeitreihe (b) des unbeeinflussten Systems $(u=0)$. Übertragungsfunktion $|T(f)|$ und Zeitreihe des geregelten ColpittsSystems (Regelung aktiviert bei $t=0$ ) für (c), (d) TDAS-Kontrolle (2.10), (e), (f) Kontrolle mit einem Kerbfilter (3.3), (g), (h) Kontrolle mit zwei Kerbfiltern (3.4).

Simulationen wurden die Differentialgleichungen

$$
\begin{aligned}
\dot{V}_{C_{1}} & =\frac{1}{R C}\left(V_{\text {in }}+\frac{2 Q-1}{Q} V_{C_{2}}-V_{C_{1}}\right) \\
\dot{V}_{C_{2}} & =\frac{1}{R C}\left(V_{\text {in }}+\frac{Q-1}{Q} V_{C_{2}}-V_{C_{1}}\right) \\
V_{\text {out }} & =\frac{1}{Q} V_{C_{2}}
\end{aligned}
$$

als Prototyp eines Kerbfilters mit dem Eingangssignal $V_{\text {in }}$ und dem Ausgangssignal $V_{\text {out }}$ verwendet. $V_{C_{1}}$ und $V_{C_{2}}$ entsprechen Kondensatorspannungen der elektronischen Realisierung der Wien-Brücke, $R C$ bestimmt die Lage der Kerbe bei der Resonanzfrequenz $f=(2 \pi R C)^{-1}$ im Fourierraum, wobei die Flankensteilheit durch die Filtergüte $Q$ angepasst werden kann. Das auf den Colpitts-Oszillator (2.26a) angewendete Regelsignal besitzt damit eine ähnliche Struktur wie das TDAS-Signal (3.1) und lautet

$$
u(t)=k\left(V_{\text {in }}(t)-V_{\text {out }}(t)\right) .
$$


unter Verwendung der $U_{C_{1}}$-Komponente aus Gl. (2.26a) als Eingangssignal $V_{\text {in }}$. Abb 3.1e zeigt die entsprechende Übertragungsfunktion der Gl. (3.3) mit den Parametern $Q=16.9$, $R=10.46 \mathrm{k} \Omega, C=0.1 \mathrm{mF}$ und $k=1$, deren Anwendung mit $k=0.75$ ebenfalls einen Periode-1-Orbit nach sich zieht (Abb. 3.1f). Wie anhand der zugehörigen Übertragungsfunktion ersichtlich, liegt keine Kerbe für $f=0$ vor, so dass NFF im Gegensatz zu TDAS an dieser Stelle invasiv ist, d. h. das Regelsignal $u(t)$ verschwindet nicht, nachdem die Zieldynamik angenommen bzw. generiert wird. Allerdings muss man ebenso zugestehen, dass die Zieldynamik in diesem Fall sehr nah an der systemeigenen liegt, die durch TDAS stabilisiert wird (Abb. 3.1d). Um die Regelung nichtinvasiv zu gestalten, kann eine Hochpassfilterung des Eingangssignals oder eine Subtraktion eines konstanten Offsets vorgenommen werden.

Die Stabilisierung von Fixpunkten kann mit NFF analog zu MDFC unter Einsatz eines zweiten, parallel geschalteten Kerbfilters herbeigeführt werden. Bei geeigneter Wahl der Filtergüten $Q_{m}$ und Resonanzfrequenzen $f_{m}=\left(2 \pi R_{m} C\right)^{-1}$ werden alle Frequenzkomponenten unterdrückt, die bei ausgeschalteter Regelung zu einer chaotischen Dynamik führen. Der Fall zweier Kerbfilter ist in Abb 3.1g dargestellt, die den Betrag der Übertragungsfunktion der Rückkopplung

$$
u(t)=k_{1}\left(V_{\text {in }}^{(1)}-V_{\text {out }}^{(1)}\right)+k_{2}\left(V_{\text {in }}^{(2)}-V_{\text {out }}^{(2)}\right)
$$

mit $k_{1}=k_{2}=0.75, Q_{1}=16.9, Q_{2}=14.9, C_{1}=C_{2}=0.1 \mathrm{mF}, R_{1}=10.46 \mathrm{k} \Omega$ und $R_{2}=3.15 \mathrm{k} \Omega$ enthält. In Abb. 3.1h ist die Stabilisierung des Colpitts-Fixpunktes in Form einer Zeitreihe zu sehen, wobei auch hier als Eingangsgröße die $U_{C_{1}}$-Komponente zum Einsatz kam. Da die Kerbfilter DC-Pegel (d. h. konstante Offsets) berücksichtigen, weicht dieser Fixpunkt wiederum etwas von dem systemeigenen des Colpitts-Oszillators (2.26a) mit $\mathbf{x}_{0} \approx(5.1759,-0.7502,0.0174)$ ab, das Regelsignal konvergiert gegen einen konstanten Wert.

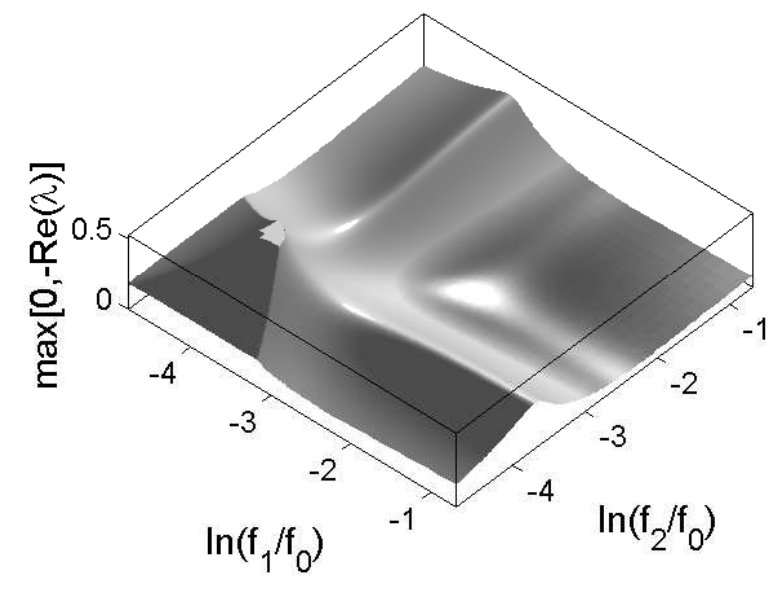

Abb. 3.2: Berechnete Stabilitätsfunktion $\max (0,-\operatorname{Re}(\lambda))$ vs. Resonanzfrequenzen $f_{1} / f_{0}$ und $f_{2} / f_{0}$ der beiden aktiven Kerbfilter (3.2),(3.4) $\left(k_{1}=\right.$ $\left.k_{2}=1.5, Q_{1}=Q_{2}=0.8\right)$ für die Fixpunktstabilisierung des ColpittsOszillators (2.26a). $\operatorname{Re}(\lambda)$ bezeichnet den größten Realteil der Eigenwerte, wobei das rückgekoppelte System stabil ist, wenn $\max (0,-\operatorname{Re}(\lambda))$ positiv ist. $f_{0}=$ $1 / T_{0}$ basiert auf der auf Seite 20 definierten natürlichen Periode des ColpittsOszillators. 
Um NFF mit zwei Kerbfiltern mit MDFC vergleichen zu können, muss wiederum eine lineare Stabilitätsanalyse in der Nähe des Fixpunktes $\mathbf{x}_{0}$ des Colpitts-Oszillators durchgeführt werden. Im Gegensatz zu MDFC führt diese Stabilitätsanalyse zu einem charakteristischen Polynom mit einer endlichen Anzahl an Eigenwerten, deren Realteile analysiert werden müssen. Das Ergebnis ist in Abb. 3.2 in Form der Stabilitätsfunktion $\max (0,-\operatorname{Re}(\lambda))$ als Maß für die Robustheit der Regelung gegen die Resonanzfrequenzen $f_{m}$ der zwei verwendeten Filter aufgetragen. $\lambda$ bezeichnet den Eigenwert mit dem größten Realteil, als Eingangssignal wurden wiederum die AC-gekoppelten Komponenten von $U_{C_{1}}$ verwendet. Der Fixpunkt wird erfolgreich stabilisiert, wenn alle Eigenwerte über einen negativen Realteil verfügen. Je höher also die Stabilitätswerte in Abb. 3.2 sind, desto stabiler ist die Regelung. Der maximale Stabilitätswert von $\approx-0.4$ ist im Bereich von MDFC und stabiler als (E)TDAS oder seinem Grenzfall des einpoligen Hochpassfilters. Die Stabilisierung gelingt in diesem Zusammenhang am besten, wenn die Resonanzfrequenzen paarweise verschieden und vor allem verschieden von den Hauptfrequenzen $\approx 0.15 f_{0}$ und $\approx 0.09 f_{0}$ (sichtbar anhand des weissen Punktes in Abb. 3.2) des dynamischen Systems gewählt werden. Dies ist verständlich, da Frequenzen, die in einer Kerbe im Frequenzraum liegen, nicht zurückgekoppelt werden. Fixpunktstabilisierung ist in dem Fall nicht möglich.

Im Gegensatz zu MDFC besteht bei NFF allerdings nicht die Möglichkeit einer Einzelgewichtung von Ein- und Ausgangssignalen (im Falle von MDFC sind das gerade die zeitverzögerten Signale). Die Auswirkungen werden beim Betrachten der jeweiligen Übertragungsfunktion deutlich. Zwar besitzt NFF durch die Filtergüte $Q$ einen weiteren Para-

Abb. 3.3: Adaptive Anpassung eines Wien Filters Gl. (3.2),(3.3). (a) Logarithmisch farbkodierte Darstellung der in Abhängigkeit von der Zeit auftretenden Frequenzen für das freilaufende Colpitts-Systems (2.26a) (Kurzzeitspektrum). (b) Betrag der Übertragungsfunktion des Wien-Filters aus Gl. (3.5), dessen Resonanzfrequenz mit Hilfe der Kondensatorspannung $U_{C_{1}}$ aus Gl. (2.26a) moduliert wird. Durch diese zusätzliche Rückkopplung reagiert das Filter je nach Vorzeichen des Parameters $\kappa$ aus Gl. (3.5) auf zeitliche Veränderungen im Frequenzspektrum. Das Regelsignal wird berechnet aber nicht angewendet (offenes System).

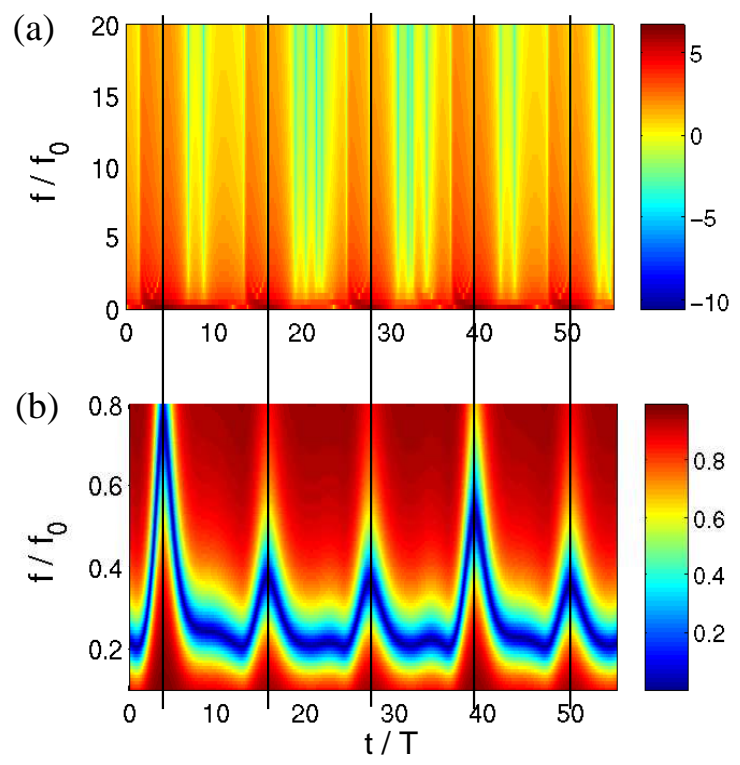

meter, die Übertragungsfunktion $|T(f)|$ verfügt aber dennoch über eine deutlich geringere 
Welligkeit als eine vergleichbare von MDFC. Folglich kann NFF für breite Frequenzbereiche nicht so flexibel an das zu regelnde dynamische System angepasst werden wie MDFC, es sei denn, die Anzahl der eingesetzten Filter wird erhöht. Ein großer Vorteil von NFF liegt allerdings in seiner relativ einfachen Realisierungsmöglichkeit durch analoge Elektronik, die auch dafür geeignet ist, schnelle chaotische Dynamik zu regeln. Eine weitere Verbesserung der Wirkungsweise von NFF bezüglich der Fixpunktstabilisierung kann durch eine aktive Anpassung der Resonanzfrequenz der verwendeten Filter erzielt werden. Das kann z. B. durch eine zusätzliche Modulation des Widerstandes $R$ durch das FilterEingangssignal geschehen. Im Detail verändert sich dann das Gleichungssystem (3.2) zu

$$
\begin{aligned}
\dot{V}_{C_{1}} & =\frac{1}{\left(R+\kappa V_{\text {in }}\right) C}\left(V_{\text {in }}+\frac{2 Q-1}{Q} V_{C_{2}}-V_{C_{1}}\right) \\
\dot{V}_{C_{2}} & =\frac{1}{\left(R+\kappa V_{\text {in }}\right) C}\left(V_{\text {in }}+\frac{Q-1}{Q} V_{C_{2}}-V_{C_{1}}\right) \\
V_{\text {out }} & =\frac{1}{Q} V_{C_{2}} .
\end{aligned}
$$

mit dem Kontrollsignal

$$
u(t)=k\left(V_{\text {in }}(t)-V_{\text {out }}(t)\right)
$$

und $R+\kappa V_{\text {in }}>0$. Das Kurzzeitspektrum ${ }^{1}$ eines chaotischen System ist keineswegs zeitlich konstant, sondern kann mitunter starken Fluktuationen unterliegen, was durch die zeitlich spektrale Darstellung des Colpitts-Oszillators in Abb. 3.3a deutlich wird. Dabei ist die Amplitude der im Spektrum auftretenden Frequenzen (logarithmisch) farbkodiert dargestellt. Durch die Selbststeuerung aus Gl. (3.5), (3.6) passt das dynamische System die Filterfrequenz jeweils an die augenblicklichen Frequenzverhältnisse an, was beispielsweise für den Betrag der Übertragungsfunktion eines Wien-Filters aus Gl. (3.5), (3.6) in Abb. 3.3b gezeigt ist. Dabei wird das Filter von dem freilaufenden System gesteuert, d. h., das Regelsignal wird zwar berechnet, aber nicht auf den Colpitts-Oszillator zurückgekoppelt. Als Eingangssignal $V_{\text {in }}$ dient die Kondensatorspannung $U_{C_{1}}$, die das Filter durch die zusätzliche Kopplung an den Widerstand $R$ dazu veranlasst, auftretenden höherfrequenten Anteilen in Form einer modifizierten Resonanzfrequenz zu folgen oder aber bei entgegengesetztem Vorzeichen des Parameters $\kappa$ diesen höherfrequenten Anteilen entgegenzuwirken. Da die für Abb. 3.3b verwendete Resonanzfrequenz $f_{m}$ des modulierten Kerbfilters im Bereich einer Hauptfrequenz des Colpitts-Oszillators (2.26a) liegt, wird in diesem Fall bei Aktivierung der adaptiven Regelung lediglich ein periodischer Orbit anstelle eines Fixpunktes stabilisiert bzw. generiert.

Für eine Fixpunktstabilisierung konnte NFF in den eigenen Experimenten sowohl mit Kerbfiltern der ursprünglichen Form (3.2), (3.3) als auch in der modifizierten Version (3.5), (3.6) erfolgreich eingesetzt werden. Die entsprechenden Resultate sind dem Abschnitt 5.6 zu entnehmen.

\footnotetext{
${ }^{1}$ Der Begriff Kurzzeitspektrum bezieht sich auf kurze Zeitreihen $(t \ll \infty)$. Im Grenzfall $t \rightarrow \infty(\mathrm{d}$. h. für lange Zeitreihen) ist das Spektrum zeitlich konstant.
} 


\section{Kapitel 4}

\section{Kontrollierbarkeit von Fixpunkten}

\subsection{Allgemeines}

Vergleichbar zu den Beschränkungen für zeitverzögerte Regelungen bei der Kontrolle periodischer Orbits [71,99] gibt es auch entsprechende Restriktionen bei der Fixpunktstabilisierung. Dabei ist zwischen zwei Arten von Fixpunkten zu unterscheiden - den durch Zeitverzögerungsmethoden stabilisierbaren [6] Strudelpunkten (Paare instabiler komplex konjugierter Eigenwerte, z. B. die äußeren Lorenz-Fixpunkte) und den Sattelpunkten (ungerade Anzahl an reellen positiven Eigenwerten, alle anderen negativ und reell oder stabil komplex konjugiert, z. B. Lorenz-Fixpunkt am Ursprung). Chang et al. [34] zeigten in diesem Zusammenhang, dass ein Hochpassfilter mit einem einzigen Pol nicht in der Lage ist, Sattelpunkte zu stabilisieren. Ähnliche Betrachtungen zum Hochpassfilter [34] lassen sich auch in [122] finden.

Im folgenden werde ich zeigen, dass diese Beschränkung mit Hilfe von MDFC durch die Verwendung von asymmetrischen Verstärkungsfaktoren bzw. durch NFF ohne ACKopplung der Eingangssignale umgangen werden kann.

\subsection{Multiple Delay Feedback Control}

Im allgemeinsten Fall koppelt die Regelung $\mathbf{u}=\left(u_{1}, u_{2}, \ldots, u_{N}\right)^{t r}$ auf alle Gleichungen des betrachteten dynamischen Systems zurück und verwendet zudem alle Systemvariablen als Eingangssignale der einzelnen Regelungen $u_{j}$. Zu Gunsten einer besseren Lesbarkeit soll allerdings an dieser Stelle von dem allgemeinsten Fall Abstand genommen und auf den Anhang verwiesen werden. Stattdessen sei der Fall einer einzigen Variablen des Zustandsvektors als Messgröße betrachtet, die auch wieder auf ihre eigene Systemdifferentialgleichung zurückkoppelt. Entsprechend der Ausführungen der vorangegangenen Kapitel 
kann dann mit Hilfe der charakteristischen Matrix die charakteristische Gleichung

$$
\begin{aligned}
S(\lambda) & =\operatorname{det}[\Delta(\lambda)]=\operatorname{det}\left[\lambda I-D \mathbf{f}\left(\mathbf{x}_{0}\right)-U\left(\mathbf{x}_{0}, \lambda\right)\right] \\
& =\lambda^{n}+\sum_{j=0}^{n-1} c_{j} \lambda^{j}+\bar{u}(\lambda) \sum_{j=0}^{n-1} d_{j} \lambda^{j}
\end{aligned}
$$

berechnet werden, wobei $\bar{u}(\lambda)$ die Laplace-Transformierte des Kontrollsignals bezeichnet. Dabei besteht die charakteristische Funktion $S(\lambda)$ aus einem rein polynomialen Anteil mit den Parametern $c_{j}$ (stammt von dem freilaufenden dynamischen System) und einem gemischten polynomial-transzendenten Anteil mit den Parametern $d_{j}$ (resultiert aus der Ankopplung des Regelsignals an das dynamische System). Da sowohl die Kontrollparameter und damit $\bar{u}$ als auch die Parameter $c_{j}, d_{j}$ endlich sind, ergibt sich der Grenzfall $\lim _{\lambda \rightarrow \infty} S(\lambda) \rightarrow \infty$. Ein betrachteter Fixpunkt ist dann stabil, wenn die Kontrollparameter so gewählt werden können, dass die Funktion $S(\lambda)$ keine Nullstellen in der rechten Hälfte der komplexen Ebene besitzt (d. h. keine Nullstellen mit positivem Realteil).

Um zu zeigen, dass dieses Ziel für Sattelpunkte nicht mit jeder Kontrollmethode erreicht werden kann, sei zunächst (E)TDAS mit

$$
\bar{u}(\lambda)=k\left(\frac{e^{-\lambda \tau}-1}{1-R e^{-\lambda \tau}}\right)
$$

betrachtet. $\bar{u}(\lambda)$ verschwindet am Ursprung ${ }^{1}$, wodurch die charakteristische Funktion an der Stelle $\lambda=0$ über den Wert

$$
S(0)=\operatorname{det}\left[-D \mathbf{f}\left(\mathbf{x}_{0}\right)\right]=(-1)^{n} \operatorname{det}\left[D \mathbf{f}\left(\mathbf{x}_{0}\right)\right]=\prod_{j=1}^{n}\left(-e_{j}\right)=c_{0}
$$

verfügt, der nur von der Jacobi-Matrix $D \mathbf{f}\left(\mathbf{x}_{0}\right)$ des $n$-dimensionalen frei laufenden Systems und seinen Eigenwerten $e_{j}$ abhängt, aber nicht von den Kontrollparametern. Bei einer ungeraden Anzahl an positiven reellen Eigenwerten tritt damit eine ungerade Anzahl an negativen Faktoren innerhalb des Produktes der Gl. (4.4) auf, was $S(0)<0$ impliziert. Da die Funktion $S(\lambda)$ bei der Einschränkung auf die reelle Achse eine kontinuierliche Funktion ist, führt die Erfüllung der beiden Randbedingungen $S(0)<0$ und $\lim _{\lambda \rightarrow \infty} S(\lambda) \rightarrow \infty$ unabhängig von den Kontrollparametern zu der Existenz eines instabilen Eigenwertes in der rechten Halbebene. D.h. (E)TDAS ist nicht in der Lage, diese Art Fixpunkte zu stabilisieren.

Wird anstelle von (E)TDAS als Regelung MDFC angewendet, so verändert sich die Laplace-Transformierte aus Gl. (4.3) gemäß

\footnotetext{
${ }^{1} \mathrm{Im}$ Gegensatz zu Sattelpunkten verhindert der Imaginärteil des laplacetransformierten Regelsignals bei der Stabilisierung Strudelpunkten ein Verschwinden des Kontrollsignals beim Übergang $\operatorname{Re}(\lambda)=0$.
} 


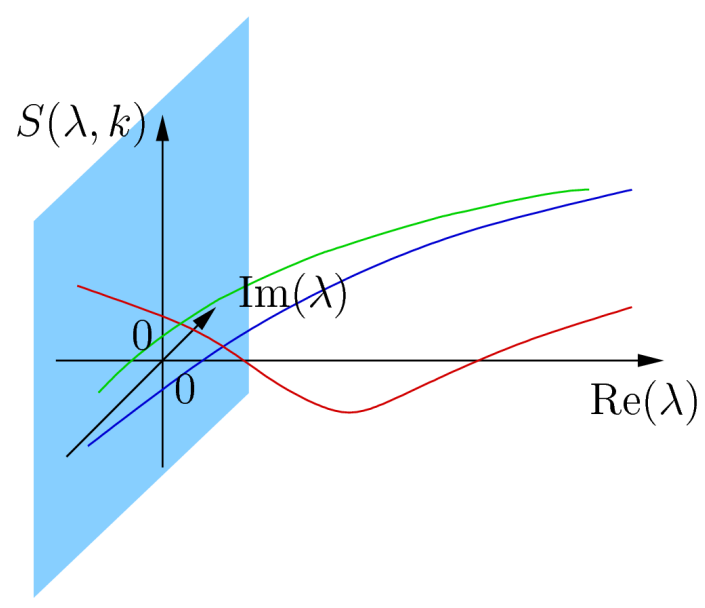

Abb. 4.1: Schematische Darstellung der Lage der instabilen rein reellen Eigenwerte im dreidimensionalen Fall. Blau: ein reeller Eigenwert $>$ 0, rot: zwei reelle Eigenwerte $>0$, grün: alle Eigenwerte sind $<0$. Da rein reelle Eigenwerte betrachtet werden, beschränkt sich die Stabilitätsanalyse auf die reelle Achse. Eine Regelung ist stabil, wenn alle Eigenwerte einen negativen Realteil und die charakteristische Funktion $S(\lambda)$ keine Nullstelle auf der reellen positiven Achse (Stabilitätsgrenze durch die blaue Ebene gekennzeichnet) besitzt. Für den Einsatz einer Regelung entsprechend Gl. (2.11) oder Gl. (4.7b) ist das im Falle eines Sattelpunktes nicht gegeben.

$$
\bar{u}(\lambda)=\sum_{m=1}^{M} k_{m a} e^{-\lambda \tau_{m}}-k_{m b} .
$$

Im Gegensatz zu Gl. (4.3) verschwindet Gl. (4.5) nicht für $\lambda=0$. Folglich hängt die charakteristische Funktion

$$
S(0)=c_{0}+\left(\sum_{m=1}^{M} k_{m a}-k_{m b}\right) d_{0}
$$

am Ursprung noch von den Differenzen der Kontrollparameter $k_{m a}-k_{m b}$ ab, was dazu verwendet werden kann, $S(0)$ zu positiven Werten zu verschieben. Da der Grenzfall $\lim _{\lambda \rightarrow \infty} S(\lambda) \rightarrow \infty$ immer noch gilt, liegt eine gerade Anzahl an Nullstellen in der rechten Halbebene für die charakteristische Funktion $S(\lambda)$ vor (eventuell auch keine Nullstelle), deren Anzahl sowohl von dem betrachteten dynamischen System als auch von den Kontrollparametern abhängt. Im Gegensatz zu dem vorher behandelten (E)TDAS-Fall ist aber damit kein fundamentales Hindernis mehr für die Stabilisierung von Sattelpunkten mit geeignet gewählten Kontrollparametern gegeben.

Aus diesem Grund sind im allgemeinsten Fall der Stabilisierung von instabilen Sattelpunkten zwei Verzögerungszeiten vonnöten. Der erste zeitverzögerte Term mit asymmetrischen (unterschiedlichen) Verstärkungsfaktoren verändert das dynamische System dahingehend, dass $S(0)>0$ gewährleistet ist. Der zweite Verzögerungsterm besteht dann z. B. aus einem (E)TDAS-Kontroller, der dieses neue, veränderte System schlussendlich stabilisiert. Eine genauere Veranschaulichung dieses Sachverhaltes bietet Abb. 4.1.

Unter Verwendung geeigneter Werte für den DC-Offset $k_{0}$ (der nicht in die Stabilitätsanalyse und damit auch nicht in die charakteristische Funktion (4.1) eingeht) in Gl. (2.16) 
ist es sogar möglich, MDFC für Fixpunkte nichtinvasiv zu gestalten, z. B. für Sattelpunkte, die nicht am Ursprung lokalisiert sind. Für Sattelpunkte am Ursprung ist dieser Schritt nicht notwendig, da keine störenden Offsets vorhanden sind, die durch die Regelung verstärkt würden. Von daher ist sogar eine invasive Kontrollmethode bei einer Fixpunktstabilisierung am Ursprung effektiv nichtinvasiv.

\subsection{Notch Filter Feedback und Hochpassfilter}

Das gleiche Problem der Stabilisierung von Sattelpunkten (ungerade Anzahl reeller positiver Eigenwerte) tritt ebenfalls bei einer ganzen Klasse von nicht zeitverzögerten Regelungen auf, zu der auch das erwähnte Hochpassfilter und NFF mit AC-Kopplung der Eingangssignale gehören. Im folgenden seien ganz allgemein Regelungssysteme der Form

$$
\dot{\mathbf{p}}=A \mathbf{p}+C \mathbf{y}
$$

mit dem Regelsignal

$$
\mathbf{u}=\mathbf{u}_{0}+k B \dot{\mathbf{p}}+A_{2} \mathbf{v}_{1} \dot{\mathbf{p}}^{t r} \mathbf{v}_{\mathbf{2}}
$$

betrachtet. $A, A_{2}(k), B$ und $C$ bezeichnen in diesem Zusammenhang Matrizen, $\mathbf{v}_{1}, \mathbf{v}_{\mathbf{2}}$ Spaltenvektoren und $\mathbf{p}$ den Zustandsvektor der Regelung. $\mathbf{y}=\mathbf{g}(\mathbf{x}(t))$ beschreibt die Messung des Systems mit dem Zustandsvektor $\mathbf{x}$. Eine lineare Stabilitätsanalyse von $\dot{\mathbf{x}}=$ $\mathbf{f}(\mathbf{x})$ am Fixpunkt $\mathbf{x}_{0}$ führt unter Verwendung der Abkürzungen $J=D_{\mathbf{x}} \mathbf{f}\left(\mathbf{x}_{0}, \mathbf{u}_{0}\right), P=$ $D_{\mathbf{u}} \mathbf{f}\left(\mathbf{x}_{0}, \mathbf{u}_{0}\right)$ und $G=D_{\mathbf{x}} \mathbf{g}\left(\mathbf{x}_{0}\right)$ auf das charakteristische Polynom

$$
S(\lambda)=\operatorname{det}\left(\begin{array}{cc}
\lambda-J & -\lambda\left(k B P-A_{2} \mathbf{v}_{1} P^{t r} \mathbf{v}_{2}\right) \\
-C G & \lambda-A
\end{array}\right)=\lambda^{N}+\sum_{j=0}^{N-1} \beta_{j} \lambda^{j},
$$

das im Fall $\lim _{\lambda \rightarrow \infty} S(\lambda)$ wegen der Beschränktheit der $\beta_{j}$ gegen $\infty$ strebt. Für $\lambda=0$ verändert sich $S(\lambda)$ unabhängig von der Verstärkung $k$ zu

$$
S(0)=\operatorname{det}\left(\begin{array}{cc}
-J & 0 \\
-C G & -A
\end{array}\right)=\prod_{j=1}^{n}\left(-e_{j}^{s}\right) \prod_{l=1}^{L}\left(-e_{l}^{r}\right)
$$

mit den Eigenwerten $e_{j}^{s}$ der Jacobi-Matrix $D \mathbf{f}\left(\mathbf{x}_{0}\right)$ am Fixpunkt $\mathbf{x}_{0}$ für das betrachtete freilaufende dynamische System. $e_{l}^{r}$ bezeichnet die Eigenwerte für den eingesetzten Regler, der als stabil angenommen wird. Demzufolge sind alle entsprechenden Eigenwerte $e_{l}^{r}<0$ und damit das zweite Produkt in Gl. (4.9) positiv - unabhängig von L. Durch die ungerade Anzahl an reellen positiven Eigenwerten ist das erste Produkt in Gl. (4.9) und damit $S(0)$ für Sattelpunkte allerdings negativ. Somit existiert analog zu dem bereits behandelten (E)TDAS-Fall mindestens ein instabiler Eigenwert, der nicht mit Hilfe der Regelparameter in den stabilen Bereich verschoben werden kann, d. h. Sattelpunkte sind 
mit dieser Klasse von Reglern, in die auch das Hochpassfilter (2.13) für $A_{2}=0$ fällt, nicht stabilisierbar. Das trifft folglich auch auf jede Regelungsmethode zu, die zum Erreichen einer nichtinvasiven Rückkopplung AC-gekoppelte (hochpassgefilterte) Eingangssignale verwendet, u. a. NFF mit einer solchen Eingangskopplung. Um dieses Ergebnis für ein Wien-Filter der Gl. (3.2),(3.3) zu stützen, sei die Messfunktion $h(x, y, z)$ als Eingangssignal $U_{\text {in }}$ des dem Wien-Filter vorgeschalteten Hochpassfilters gewählt. Mit $\dot{\mathbf{x}}=\mathbf{f}(\mathbf{x})+\mathbf{u}$ folgt unter Annahme von

$$
\dot{U}_{i n}=\partial_{x} h \dot{x}+\partial_{y} h \dot{y}+\partial_{z} h \dot{z}=(\nabla h)^{t r} \dot{\mathbf{x}}=(\nabla h)^{t r} D \mathbf{f}_{\mathbf{x}} \mathbf{x}+(\nabla h)^{t r} \mathbf{x}_{0}+(\nabla h)^{t r} D \mathbf{f}_{u} u
$$

wodurch sich wiederum unter Berücksichtigung von Gl. (2.13), (4.7a), (4.7b) die Matrizen und Vektoren

$$
\begin{array}{rlrl}
A & =\left(\begin{array}{ccc}
b_{1} & 0 & b_{2} \\
\omega & -\omega & b_{3} \\
\omega & -\omega & b_{4}
\end{array}\right), & B=\left(\begin{array}{ccc}
0 & 0 & 0 \\
0 & -1 / \omega & 2 / \omega \\
0 & 0 & 0
\end{array}\right), & C=\left(\begin{array}{ccc}
c_{1} & c_{2} & c_{3} \\
0 & 0 & 0 \\
0 & 0 & 0
\end{array}\right), \\
A_{2}=\left(\begin{array}{ccc}
0 & 0 & 0 \\
-2 k & k & 0 \\
0 & 0 & 0
\end{array}\right), & \mathbf{v}_{1}=\left(\begin{array}{c}
0 \\
-1 / \omega \\
0
\end{array}\right), \quad \mathbf{v}_{2}=\left(\begin{array}{l}
0 \\
1 \\
0
\end{array}\right)
\end{array}
$$

mit den Abkürzungen $b_{1}=-\omega_{0}+\partial_{U_{\text {in }}^{\text {ac }}}(\nabla h)^{t r} D \mathbf{f}_{u} u, b_{2}=\partial_{U_{C_{2}}}(\nabla h)^{t r} D \mathbf{f}_{u} u, b_{3}=\omega(2 Q-$ $1) / Q, b_{4}=\omega(Q-1) / Q$ und $c_{j}=\partial_{x_{j}}(\nabla h)^{\operatorname{tr}} D \mathbf{f}_{x_{j}}, j \in\{1,2,3\}$ ergeben. $\omega$ beschreibt die Resonanzfrequenz des Filters und $\omega_{0}$ die Grenzfrequenz der Eingangskopplung. Mit $\mathbf{u}_{0}=0$ erfüllt das betrachtete Kerbfilter mit hochpassgefiltertem Eingangssignal dann die Gl. (4.7a), (4.7b), womit das Scheitern bei der Stabilisierung eines Sattelpunktes ersichtlich ist. Dieses Resultat ist auch auf $m$ parallel geschaltete Kerbfilter mit ACEingangskopplung verallgemeinerbar.

Die kritische Stelle, die zu diesem Ergebnis führt, ist durch die AC-Kopplung der Eingangssignale gegeben, um die Regelungsmethode nichtinvasiv zu gestalten. Wird anstelle der AC-(Hochpass)-Kopplung die Differenz des Messsignals und seinem fest eingestellten DC-Wert als Eingangssignal der Kerbfilter verwendet, so hat der oben gegebene Beweis keine Gültigkeit mehr. Im Gegenteil, es ist dann auch möglich, mit NFF instabile Sattelpunkte zu stabilisieren. Ob das am Ende auch gelingt, hängt von dem betrachteten dynamischen System ab. Einen Spezialfall stellen Systeme dar, die über Sattelpunkte am Ursprung verfügen. In diesem Fall ist auch eine nichtinvasive Regelung mit NFF ohne AC-gekoppelte Eingangssignale und eine entsprechende Stabilisierung des betrachteten Sattelpunktes möglich. Dieser Sachverhalt ist für das bekannte Lorenz-System

$$
\begin{aligned}
& \dot{x}=-\sigma(x-y) \\
& \dot{y}=r x-y-x z+u(t) \\
& \dot{z}=-b z+x y .
\end{aligned}
$$

mit den Standardparameterwerten $\sigma=10, b=8 / 3$ und $r=28$ gegeben. Dabei wird eine Rückkopplung betrachtet, die von der $y$-Variablen abhängt und auch wieder auf 
Abb. 4.2: Der Lorenzattraktor (4.11) für die Parameterwerte $\sigma=10, b=8 / 3$, $r=28$ und den drei systemeigenen Fixpunkten, lokalisiert bei $\mathbf{x}_{0 a}=(0,0,0)$ und $\mathbf{x}_{0 b, c}=( \pm 8.48, \pm 8.48,27)$. Der Fixpunkt am Ursprung ist ein Sattelpunkt (ein reeller Eigenwert > 0, ein Paar stabiler komplex konjugierter Eigenwerte), die beiden äußeren sind Strudelpunkte (ein instabiles Paar komplex konjugierter Eigenwerte, ein rein reeller $<0$ )

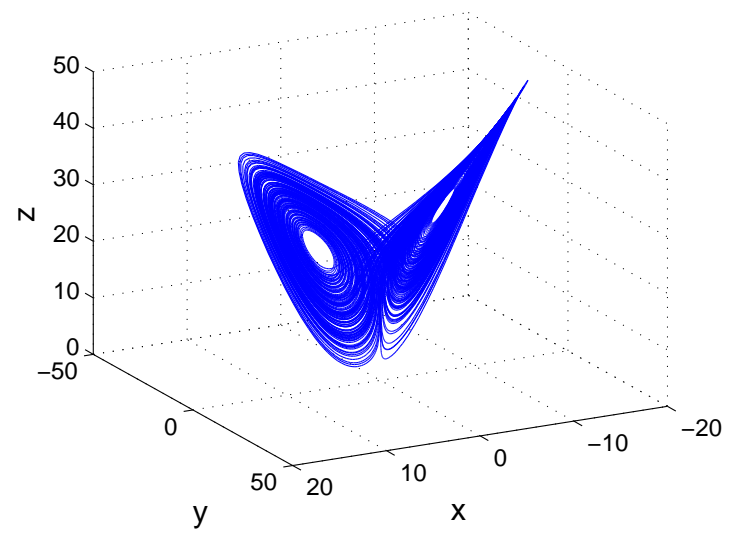

diese zurückwirkt, d. h. $\mathbf{u}=(0, u(t), 0)^{t r}$. Unter Einsatz von NFF und Verwendung von $m$ Kerbfiltern ist das charakteristische Polynom an der Stelle $\lambda=0$ im Gegensatz zu Gl (4.9) durch

$$
S(0)=\prod_{m=1}^{M} \omega_{m}^{2} \operatorname{det}\left(\begin{array}{ccc}
-a_{11} & -a_{12} & -a_{13} \\
-a_{21} & -a_{22}+\sum_{m} k_{m} & -a_{23} \\
-a_{31} & -a_{32} & -a_{33}
\end{array}\right),
$$

gegeben. Dabei besteht durch die Verstärkungsfaktoren $k_{m}$ direkter Einfluss auf die Eigenwerte der Jacobi-Matrix $D \mathbf{f}\left(\mathbf{x}_{0}\right)$ des ursprünglichen dynamischen Systems mit den Einträgen $a_{m n}$. Um die Stabilität des untersuchten Sattelpunktes zu gewährleisten, muss $\sum_{m} k_{m}$ so gewählt werden, dass als notwendige Bedingung $S(0)>0$ gültig ist. Im einzelnen führt diese Forderung auf die Bedingung

$$
\sum_{m} k_{m}>a_{22}+\frac{a_{32}\left(a_{11} a_{23}-a_{13} a_{21}\right)-a_{12}\left(a_{23} a_{31}-a_{21} a_{33}\right)}{a_{13} a_{31}-a_{11} a_{33}}
$$

für $a_{13} a_{31}-a_{11} a_{33}>0$ sowie auf

$$
\sum_{m} k_{m}<a_{22}+\frac{a_{32}\left(a_{11} a_{23}-a_{13} a_{21}\right)-a_{12}\left(a_{23} a_{31}-a_{21} a_{33}\right)}{a_{13} a_{31}-a_{11} a_{33}}
$$

für den Fall $a_{13} a_{31}-a_{11} a_{33}<0$. Die Richtigkeit dieser Ergebnisse zeigt z. B. Abb. 4.3a. Dabei führt die Anwendung von NFF bestehend aus zwei Wien-Filtern mit den Parametern $k_{1}=k_{2}=15, Q_{1}=Q_{2}=1$ sowie $f_{1}=2.12$ und $f_{2}=2.45$ zur nichtinvasiven Stabilisierung des Sattelpunktes am Ursprung. Eine lineare Stabilitätsanalyse enthüllt ausgedehnte Stabilitätsgebiete in Abhängigkeit der Filter-Resonanzfrequenzen $f_{1,2}$, wobei zur Visualisierung die Stabilitätsfunktion $\max (0,-\operatorname{Re}(\lambda))$ in Abb. 4.3b zum Einsatz kommt. $\operatorname{Re}(\lambda)$ bezeichnet den Eigenwert mit dem größten Realteil, d. h. je höher die Werte, desto stabiler ist die Regelung, wobei die Verstärkungen $k_{1}, k_{2}$ entsprechend hoch gewählt sein müssen.

Dieses Resultat kann noch weiter für Sattelpunkte verallgemeinert werden, die nicht am Ursprung lokalisiert sind. Für diesen Zweck sollte als Eingangssignal für die Kerbfilter die 
(a)
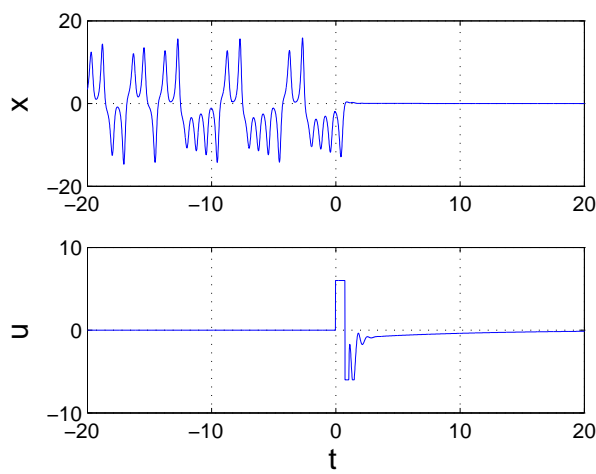

(b)

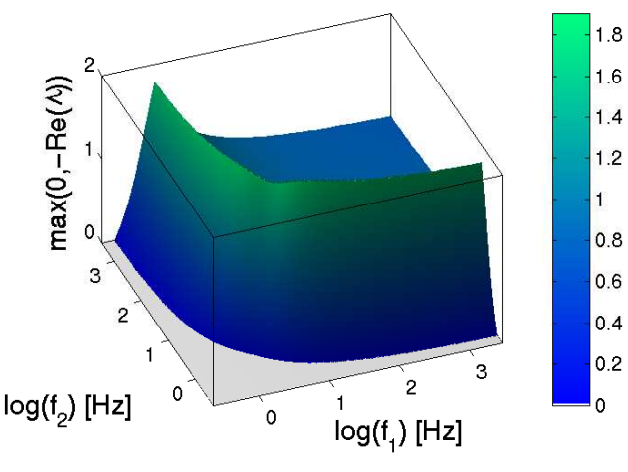

\begin{abstract}
Abb. 4.3: Stabilisierung des Sattelpunktes $(0,0,0)$ des Lorenzsystems (4.11) mit Hilfe von NFF bestehend aus zwei Wien-Filtern (3.2),(3.4). (a) Zur Zeit $t=0$ wurde die Regelung aktiviert, wobei das Regelsignal auf eine Amplitude von \pm 8 begrenzt wurde. Die Güte wurde zu $Q_{j}=1$, die Verstärkungen zu $k_{j}=13.5$ für beide Filter gewählt. Das Nachschwingen des Regelsignals bei Erreichen des Fixpunktes erklärt sich aus den noch geladenen Kondensatoren der Kerbfilter. (b) Stabilitätsdiagramm des entsprechenden Sattelpunktes. Dargestellt ist die Stabilitätsfunktion $\max (0,-\operatorname{Re}(\lambda))$ vs. Resonanzfrequenzen $f_{1}$ und $f_{2}$ der beiden aktiven Kerbfilter $\left(k_{1}=k_{2}=15, Q_{1}=Q_{2}=1\right)$.
\end{abstract}

Differenz aus der Messfunktion $\mathbf{g}(\mathbf{x}(t))^{(\mathrm{dc})+(\mathrm{ac})}$ und ihrem DC-Pegel $\mathbf{g}(\mathbf{x}(t))^{(\mathrm{dc})}$ dienen, der wiederum statisch einzustellen ist. So kann z. B. in einem ersten Schritt bei abgeschalteter Regelung dieser Pegel durch einen Tiefpassfilter ermittelt und dann für die Regelung fest eingestellt werden.

Ergebnis dieser Betrachtungen ist, dass es bei der Stabilisierung von instabilen Sattelpunkten in besonderer Weise darauf ankommt, wie man die Messsignale und die Ankopplung an den Regler wählt. An dieser Stelle ist explizit darauf hinzuweisen, dass nur stabile Regelungen betrachtet wurden, was für die Stabilisierung von periodischen Orbits nicht der einzige Weg ist. Häufig werden diese durch Anwendung von (E)TDAS (2.11) stabilisiert, wobei man bei der allgemeinen experimentellen Situation allerdings nicht weiß, wie die externe Kraft an die inneren Freiheitsgrade koppelt. Deswegen ist auch nicht immer klar, wie die eigentliche Stabilisierung instabiler Orbits überhaupt erreicht wird. Erst kürzlich wurde durch Pyragas et al. [124] eine mathematische Begründung für den Erfolg der TDAS-Rückkopplung für Systeme gegeben, die eine subkritische Hopf-Bifurkation durchlaufen haben. Unter diesem Gesichtspunkt sind periodische Orbits bis heute Gegenstand intensiver Forschung. 


\subsection{Periodische Orbits}

Generell wurde in $[71,73,99,101]$ gezeigt, dass für eine erfolgreiche Stabilisierung von periodischen Orbits mittels zeitverzögerter Regelung das Vorhandensein von Torsion essentiell ist (Eine Illustration dieser Torsionsbewegung um den Orbit $\xi(t)$ ist der Abb. 4.4 zu entnehmen.). Das bedeutet, dass keine Orbits stabilisiert werden können, die eine ungerade Anzahl an positiven Floquet-Exponenten mit Betrag $>1$ aufweisen, da eine endliche Frequenz nur von einem Paar von Eigenwerten generiert werden kann [140]. Um diese besondere Art periodischer Orbits zu stabilisieren, wird in [118,123] die Einführung eines instabilen Freiheitsgrades, d. h. eines instabilen Reglers $\mathbf{u}_{j}(t)$ der Form

$$
\begin{aligned}
\mathbf{u}_{j}(t) & =\mathbf{u}(t)+\mathbf{p}(t) \\
\dot{\mathbf{p}}(t) & =\lambda_{c}^{0} \mathbf{p}(t)+\left(\lambda_{c}^{0}-\lambda_{c}^{\infty}\right) \mathbf{u}(t)
\end{aligned}
$$

vorgeschlagen. Für Vorfaktoren $\lambda_{c}^{0}>0$ und $\lambda_{c}^{\infty}>0$ können als Rückkopplungssignal $\mathbf{u}(t)$

Abb. 4.4: Schematische Darstellung der Torsionsbeschränkung für Zeitverzögerungsregelungen bezüglich periodischer Orbits. Stabilisierbar sind mit solchen Methoden ((E)TDAS, NTDAS, MDFC) nur Orbits mit $\omega \neq 0$.

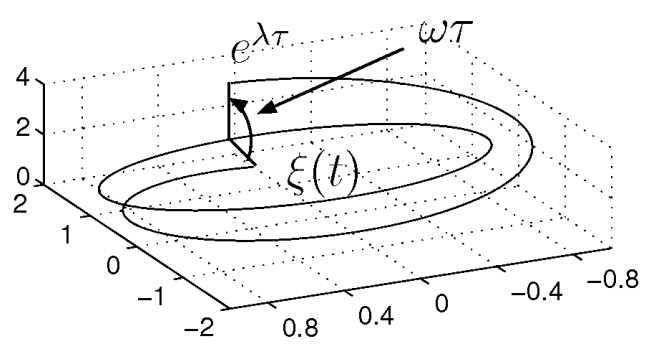

Regelungen des (E)TDAS-Typs (2.11) oder andere der Torsionsbechränkung unterliegende Regelungsstrategien verwendet werden, die auf diese Weise doch direkten Einfluss auf reelle (positive) Eigenwerte nehmen können. Im wesentlichen handelt es sich bei dem instabilen Freiheitsgrad um eine zusätzliche weitere Tiefpassfilterung des Regelsignals, wobei allerdings der Widerstand dieses Tiefpassfilters "negativ "zu wählen ist $\left(\lambda_{c}^{0}\right.$ entspricht der Grenzfrequenz $\left.-\omega_{0}\right)$. Das kann im Experiment z. B. dadurch erreicht werden, indem der übliche Widerstand des Tiefpasses durch einen NIC ${ }^{2}$ ersetzt wird. Bedingt durch die Instabilität des Reglers ist bei der Einstellung der Parameter allerdings darauf zu achten, dass ein experimentelles System bei einer schlechten Parameterwahl keinen Schaden nimmt bzw. andere Komplikationen auftreten. Dieser Sachverhalt wird auch durch [100] gestützt, wo ein Beweis dafür gegeben wird, dass die Einführung eines instabilen Freiheitsgrades eben nicht ohne weiteres für zeitkontinuierliche Systeme zum Erfolg führt. Diese Aussage wird auch von [55] für räumlich ausgedehnte Systeme untermauert, auf die im sechsten Kapitel noch eingegangen werden wird.

\footnotetext{
${ }^{2}$ Bei einem NIC handelt es sich um eine Operationsverstärker-Schaltung, die einen negativen Widerstand simuliert. Das wird durch die Einführung einer Phasenverschiebung von $\pi$ zwischen Spannung und Strom erreicht [148].
} 


\section{Kapitel 5}

\section{Kontrolle chaotischer Laserdynamik}

\subsection{Holografische Displays}

In einigen Jahren werden holografische Displays Anzeigemedien revolutionieren. Zum Einsatz kommen dabei farbige Laser (rot, blau, grün), die zum Übermitteln der Bildinformationen in ihrer Lichthelligkeit einzeln moduliert werden. Der eigentliche Bildaufbau wird ähnlich wie beim Fernseher über eine zeilenweise Rasterung erfolgen und erfordert eine konstante Grundintensität (cw) der Laser, um unerwünschte HellDunkel-Effekte (Abb. 5.1) zu vermeiden. Durch die Kohärenz der Laserstrahlung und den Vorgang der Holografie erwecken diese holografischen Displays einen dreidimensionalen Bildeindruck, wodurch wegen der Winkelabhängigkeit der Holografie zudem unterschiedliche Informationen in verschiedenen Raumrichtungen angezeigt werden können, was effektiv zu einer Steigerung der Informationsdichte führen wird. Genauso wichtig wie die Dreidimensionalität des Bildes ist allerdings die Tatsache, dass das Bild im Gegensatz zu einem z. B. anhand eines Beamers erzeugten Bildes im-
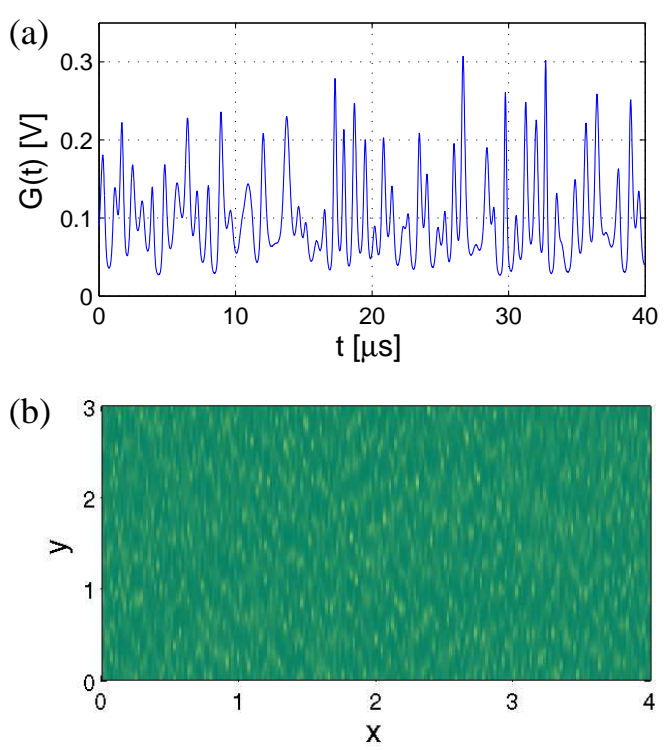

Abb. 5.1: Zeilenweiser Bildaufbau mit der (a) chaotischen Laserintensität $G(t)$ führt zu (b) Hell-Dunkel-Effekten. mer scharf ist, unabhängig vom Abstand der Projektionsanlage zum Projektionshintergrund. Demzufolge muss bei einem holografischen Display auch der Projektionshintergrund nicht mehr glatt sein, was auch die Pro- 
jektion von scharfen und (bedingt durch den Lasereinsatz) farbbrillanten Bildern z. B. auf einen Wasserfall ermöglicht.

Zur Zeit arbeiten verschiedene Hersteller an der Entwicklung eines solchen Systems. Die Laser, die dabei zum Einsatz kommen, sind aber bis jetzt nicht sonderlich kompakt, so dass das gesamte System derzeit den Abmessungen eines großen Kühlschrankes entspricht. Das ist natürlich im Zeitalter einer immer weiter fortschreitenden Miniaturisierung nicht wünschenswert und von daher ist der Einsatz von kleinen, leistungsstarken, kompakten Halbleiter- bzw. Festkörperlasern vorgesehen. Wegen der hohen Besetzungsdichte in der Nähe der Fermi-Energie eignen sich Festkörperlaser im Vergleich zu Gaslasern besonders gut zur Erzeugung höherer Intensitäten bei zugleich kleinerer Bauart. Allerdings wird es auf lange Sicht keine Festkörper-Verbindung geben, die einen Laserübergang im grünen Spektralbereich zulässt, so dass (Festkörper)Laser für die Erzeugung grünen und blauen Laserlichtes vorgesehen sind, die sich des nichtlinearen Effektes der Frequenzverdopplung bedienen.

\section{$5.2 \quad$ Frequenzverdopplung}

In der linearen Optik gilt zum einen das Superpositionsprinzip ${ }^{1}$ und zum anderen die Frequenzerhaltung $^{2}$, wodurch der Absorptionskoeffizient, die Polarisation und somit auch die Brechzahl nur von der Wellenlänge des auf ein Medium einfallenden Lichtes und nicht von dessen Intensität abhängt. Durch diese Anregung werden die Elektronen innerhalb der Materie gegenüber den schwereren Atomkernen verschoben. Die dadurch entstandenen Dipole (Polarisation) strahlen ein elektrisches Feld ab, das sich dem ursprünglich anregenden Feld überlagert und die Antwort des betrachteten Mediums auf die äußere Anregung darstellt. Als Folge davon ergibt sich eine geringere Ausbreitungsgeschwindigkeit innerhalb des Mediums, was durch die Brechzahl beschrieben wird. Ist die eingestrahlte Feldstärke groß, so treten zusätzliche anharmonische Schwingungen auf, da die Elektronen zwar durch die äußere Anregung beliebig weit von ihren Kernen entfernt, aber nur bis zu einer bestimmten Distanz innerhalb der Materie, z. B. eines Frequenzverdopplerkristalls zu ihren Kernen gedrückt werden können. Im einfachsten Fall kann eine Taylorentwicklung für die Polarisation

$$
\mathbf{P} \approx \epsilon_{0}\left(\chi^{(1)} \mathbf{E}+\chi^{(2)} \mathbf{E}^{2}\right)
$$

mit der Suszeptibilität $\chi$, der Dielektrizitätskonstante $\epsilon_{0}$ und der Feldstärke $\mathbf{E}$ angesetzt werden. Lässt man für einen kurzen Moment die Phasenverschiebung zwischen einer einfallenden Welle $\mathbf{E}=\mathbf{E}_{\mathbf{0}} \cos (\omega t)$ und der Polarisation $\mathbf{P}$ außer acht und berücksichtigt zudem nur den ersten nichtlinearen Term der Taylorentwicklung, so ergibt sich mit dem

\footnotetext{
${ }^{1}$ Wellen überlagern sich unabhängig voneinander, es findet keine gegenseitige Beeinflussung statt.

${ }^{2}$ Bei der Wechselwirkung zwischen Licht und Materie werden keine neuen Lichtfrequenzen generiert.
} 
Additionstheorem

$$
\cos ^{2}(\omega t)=\frac{1}{2}(1-\cos (2 \omega t))
$$

der Ausdruck

$$
\begin{aligned}
\mathbf{P} & \approx \epsilon_{0}\left(\chi^{(1)} \mathbf{E}_{\mathbf{0}} \cos (\omega t)+\chi^{(2)} \mathbf{E}_{\mathbf{0}}^{2} \cos ^{2}(\omega t)\right) \\
& =\underbrace{\epsilon_{0} \chi^{(1)} \mathbf{E}_{\mathbf{0}} \cos (\omega t)}_{\mathbf{P}_{\text {linear }}(\omega)}+\underbrace{\frac{\epsilon_{0} \chi^{(2)}}{2} \mathbf{E}_{\mathbf{0}}{ }^{2}}_{\mathbf{P}(0)}-\underbrace{\frac{\epsilon_{0} \chi^{(2)}}{2} \mathbf{E}_{\mathbf{0}}{ }^{2} \cos (2 \omega t)}_{\mathbf{P}_{\mathrm{nl}}(2 \omega)} \\
& =\mathbf{P}(0)+\mathbf{P}_{\text {linear }}(\omega)+\mathbf{P}_{\mathrm{nl}}(2 \omega),
\end{aligned}
$$

der über einen Anteil mit der doppelten Frequenz verfügt (Frequenzverdopplung, shg, second harmonic generation). Einen Schwellwert gibt es für diesen Prozess, der durch das Auftreten der anharmonischen Terme die Prinzipien der Superposition und der Frequenzerhaltung außer Kraft setzt, nicht. Für eine genauere Herleitung der Differentialgleichungen für die 3-Wellen-Wechselwirkung, aus denen auch der Vorgang der Frequenzverdopplung extrahiert werden kann, sei auf [82] verwiesen. Dabei existieren mit der internen und der externen Verdopplung zwei Realisierungsmöglichkeiten, wobei allerdings die interne Verdopplung der externen vorzuziehen ist, da die Intensität der z. B. grünen Laserstrahlung bis zum heutigen Tag wegen der höheren Feldstärke innerhalb des Resonators größer ist. Zudem wäre bei externer Verdopplung ein weiterer Resonator notwendig, was zu höheren Kosten und einem gesteigerten (Justier-)Aufwand führen würde und damit die interne Verdopplung favorisiert. Beide Möglichkeiten wurden in den 1970er Jahren bereits probiert, allerdings war die erzeugte grüne Laserstrahlung zunächst nur von geringer Intensität. Die Effizienz konnte aber für beide Arten in den 1980ern durch genau abgestimmte Pumpdioden mit höherer Leistung und angepassten (Verdoppler-)Kristallen deutlich verbessert werden. Diese Kristalle besitzen eine möglichst hohe nichtlineare Suszeptibilität und eine hohe optische Belastbarkeit, damit auch große Intensitäten eingestrahlt werden können. Weitere Bedingung einer effizienten Umwandlung von z. B. infrarotem in grünes Licht ist neben einer guten Transparenz für beide Wellenlängen auch eine identische Ausbreitungsgeschwindigkeit der eingestrahlten Grund- und der frequenzverdoppelten Oberwelle. Das ist im Normalfall nicht möglich, da die Brechzahl sowohl winkel- als auch frequenzabhängig ist. Es gibt aber spezielle doppelbrechende Kristalle, für die unter einem bestimmten Winkel $\theta$ (gemessen zur optischen Achse des Kristalls) diese sogenannte Phasenanpassung (phase matching) $n(\omega)=n(2 \omega)$ relativ gut erfüllt ist. Aufgrund der festen Phasenbeziehung zwischen Polarisationswelle und frequenzverdoppelter Oberwelle kommt es dann durch Überlagerung zu der geforderten Leistungssteigerung der Oberwelle. Die Umwandlung verbessert sich zudem mit wachsender Länge des Verdopplerkristalls (meistens KTP oder KDP), wonach rein theoretisch bei entsprechender Länge des Kristalls die gesamte Energie der eingestrahlten Grundwelle in die 2. Harmonische überführt werden könnte. In der Praxis ist das aber nicht der Fall, da die Wellen in einem realen Kristall mit wachsender Kristalllänge auseinanderlaufen. Das liegt zum einen an Störungen im 
Kristall und zum anderen daran, dass das eingestrahlte Laserlicht keine reine Sinuswelle darstellt.

Diese Grundwelle wird in den allermeisten Fällen von einem Festkörperlaser, z. B. einem Nd:YAG Laser generiert. Dabei handelt sich um einen chemisch relativ inaktiven und damit alterungsbeständigen Yttrium-Aluminium-Granat-Wirtskristall $\left(\mathrm{Y}_{3} \mathrm{Al}_{5} \mathrm{O}_{12}\right)$, in dem die laseraktiven $\mathrm{Nd}^{3+}$-Ionen eingelagert sind. Der Wirtskristall verfügt über mehrere Pumpbänder, von denen die angeregten Elektronen schnell und strahlungslos in das metastabile ${ }^{4} \mathrm{~F}_{3 / 2}$-Niveau übergehen. Die Verweilzeit beträgt im Durchschnitt $240 \mu \mathrm{s}$, der Fluoreszenzwirkungsgrad mehr als $99,5 \%$. Bei dem Übergang auf das ${ }^{4} \mathrm{I}_{9 / 2}$-Niveau wird dann die am häufigsten für diesen Laser auftretende Wellenlänge von $1064 \mathrm{~nm}$ emittiert, wobei durch Variation der Resonatorbedingungen allerdings auch die Abgabe von Strahlung der Wellenlänge $\lambda=900 \mathrm{~nm}$ und $\lambda=1350 \mathrm{~nm}$ möglich ist. Um eine intensive Grundwelle zu erhalten, wird der YAG-Laser seinerseits oft durch Diodenarrays mit einem engen Spektralbereich gepumpt, deren emittierte Strahlung gerade Übergänge in die relevanten Laserniveaus zulassen.

Diese technischen Fortschritte führten schließlich Mitte der 1980er Jahre zu den von Baer beobachteten (chaotischen) Intensitätsschwankungen intern frequenzverdoppelter Laser, wenn ein bestimmter, kritischer Wert z. B. für den Pumpstrom oder die Temperatur überschritten wird [16, 126]. Gerade diese chaotische Dynamik ist für Anwendungen problematisch, was zusammen mit der Tatsache, dass frequenzverdoppelte Nd:YAG Laser häufig als Quelle kohärenten grünen Lichtes verwendet werden, als green problem in die Literatur einging.

\subsection{Lasermodell}

Zur Simulation dieser Dynamik wurde das von Pyragas et al. [121] veröffentlichte und auf den umfangreichen Kenntnissen einer langen Reihe von Lasermodellen [30, 68, 84, 119, 155] basierende Modell eines intern frequenzverdoppelten Festkörperlasers verwendet. Die Zustandsgleichungen für die optischen Moden werden durch

$$
\begin{gathered}
\frac{\mathrm{d} I_{j}}{\mathrm{~d} \vartheta}=\left[G_{j}-\frac{\epsilon}{\alpha \eta}\left(\mathrm{g}\left(I_{j}-I_{j}^{0}\right)+2 \sum_{l \neq j} \mu_{l j}\left(I_{l}-I_{l}^{0}\right)\right)\right] I_{j} \\
\frac{\mathrm{d} G_{j}}{\mathrm{~d} \vartheta}=\Delta \mathrm{w}\left\langle u_{j}(x)\right\rangle+\mathrm{w}_{0} \sum_{l} \beta_{l j}\left(I_{l}^{0}-I_{l}\right)-\eta G_{j}-\eta \sum_{l m} T_{l j m} I_{l} G_{m}
\end{gathered}
$$

beschrieben, wobei $I_{j}$ die Intensität der $j$-ten Mode und $G_{j}$ die entsprechende Kleinsignalverstärkung bezeichnet. Im weiteren Verlauf wird die systemimmanente Zeit durch $\vartheta=t / T$ auf dimensionslose Größe reskaliert. Dabei ist $T=\sqrt{\tau_{c} \tau_{f} / \alpha} \approx 2.19 \mu$ s eine 
charakteristische Zeitskala des Lasers, die durch die Umlaufzeit $\tau_{c}=2 L / c$ im Laserresonator der Länge $L$ und die obere Grenze der Fluoreszenzzeit $\tau_{f}=240 \mu$ s determiniert ist. Die später zu untersuchenden Fluktuationen der Ausgangsintensität spielen sich auf der Zeitskala der Relaxationsschwingungen des Lasersystems ab, die das Ergebnis des Energieaustausches zwischen den Atomen der Laserniveaus und der Strahlung innerhalb des Resonators sind. Die Frequenz der Oszillationen hängt u. a. von den Resonator-Verlusten $\alpha_{j}$, der Fluoreszenz und dem nichtlinearen Koeffizienten $\epsilon$ des KTP-Kristalls ab [63], durch dessen Einbringen in den Laserresonator die normalerweise stark gedämpften Relaxationsschwingungen entdämpft werden. Als Folge davon wird der Laser bei Überschreiten einer bestimmten kritischen Pumprate $\mathrm{w}_{0}$ instabil. Die Pumprate

$$
\mathrm{w}=\mathrm{w}_{0}+\Delta \mathrm{w}
$$

wird in diesem Modell durch einen fest vorgewählten Teil $\mathrm{w}_{0}$ und einen variablen Teil $\Delta \mathrm{w}$ beschrieben. Gerade der variable Teil $\Delta \mathrm{w}$ ist im Anschluss hieran wichtig für die Stabilisierung des Lasers, da über ihn die Regelung und eine eventuelle, zusätzliche Modulation auf den Pumpstrom des Lasers wirkt. Die Gl. (5.5a),(5.5b) beschreiben den wohl am verbreitetsten experimentell vorliegenden Fall eines von einem Ende her gepumpten frequenzverdoppelten Festkörperlasers, wobei das Pumpen als inhomogen bezeichnet werden muss, da der YAG- und der Verdopplerkristall nicht den gesamten Laserresonator ausfüllen. g bezeichnet einen geometrischen Faktor, dessen Wert von der optischen Phasenverzögerung durch den Verstärkungs- und den Verdopplerkristall sowie deren Winkel der schnellen Achsen zueinander herrührt. Beide Kristalle sind doppelbrechend, sodass für die Simulationen ein Wert von $\mathrm{g}=0.1$ angenommen wird. Weiterhin muss die Kreuzsättigung, die in Beziehung zu dem Wettkampf der verschiedenen longitudinalen Moden untereinander steht, im Lasersystem berücksichtigt werden, was durch die Matrix $\left\{\beta_{l j}\right\}$ geschieht. Verfügen die Moden $l$ und $j$ über dieselbe Polarisationsrichtung, so ist $\mu_{l j}=\mathrm{g}$; ist die Mode $l$ senkrecht zur Mode $j$ polarisiert, so gilt $\mu_{l j}=1-\mathrm{g}$. Dem nichtlinearen Effekt der Verdopplung wird durch den nichtlinearen Koeffizient $\epsilon=2 \cdot 10^{-5}$ Rechnung getragen, dessen Wert von den Eigenschaften des KTP-Kristalls abhängt. Weiterhin wird für alle Moden der gleiche Verlustwert $\alpha=0.01$ angenommen, $\eta$ berechnet sich schließlich zu $\eta=\sqrt{\tau_{c} /\left(\tau_{f} \alpha\right)}$. Anschaulicher als der Begriff der Kleinsignalverstärkung ist die Besetzungsinversion $n$. Ähnlich wie die Pumprate kann auch sie in einen festen und einen variablen Anteil

$$
n=n_{0}+\eta \varphi
$$

entwickelt werden, wobei die Funktion $\varphi$ und damit auch die Besetzungsinversion $n$ durch die Größen $G_{j}$, die symmetrische Matrix $\left\{\beta_{l j}\right\}$ und den Tensor $\left\{T_{l j k}\right\}$ in die Berechnungen einfließt. Die Gleichung (5.5b) für die Kleinsignalverstärkungen entstand nach Linearisierung bei der konstanten Pumprate $\mathrm{w}_{0}$. Weitere Details des Modells können [121] entnommen werden.

$I_{j}^{0}$ bezeichnet die Fixpunktintensitäten unter der Voraussetzung, dass die Gln.(5.5a) und $(5.5 \mathrm{~b})$ in der Nähe des Fixpunktes für $\mathrm{w}=\mathrm{w}_{0}, I_{j}=I_{j}^{0}, G_{j}=0$ erfüllt sind. Nebenbedin- 
gung für die Berechnung der Fixpunktintensitäten ist

$$
\mathrm{w}_{0}\left\langle\frac{u_{j}(x)}{1+\sum_{l} I_{l}^{0} u_{l}(x)}\right\rangle-1-\frac{\epsilon}{\alpha}\left(\mathrm{g} I_{j}^{0}+2 \sum_{l \neq j} \mu_{l j} I_{l}^{0}\right)=0,
$$

was sich im expliziten Drei-Moden-Fall als

$$
\begin{array}{r}
\left(\frac{3}{2}+\frac{\epsilon \mathrm{g}}{\alpha \mathrm{w}_{0}}\right) I_{0}^{0}+\left(\left(2+K_{1}\right) g_{1}+\frac{4 \epsilon(1-\mathrm{g})}{\alpha \mathrm{w}_{0}}\right) I_{0}^{1}=1-\frac{1}{\mathrm{w}_{0}} \\
\left(1+\frac{K_{1}}{2}+\frac{2 \epsilon(1-\mathrm{g})}{\alpha \mathrm{w}_{0} g_{1}}\right) I_{0}^{0}+\left(\left(2+\frac{1+K_{2}}{2}\right) g_{1}+\frac{3 \epsilon \mathrm{g}}{\alpha \mathrm{w}_{0} g_{1}}\right) I_{1}^{0}=1-\frac{1}{\mathrm{w}_{0} g_{1}}
\end{array}
$$

unter Verwendung von

$$
g_{l}=\frac{L^{2} \Delta \nu^{2}}{L^{2} \Delta \nu^{2}+c^{2} l^{2}}
$$

und

$$
u_{l}(x)=g_{l}[1-\cos (2 \pi(M+l) x)]
$$

äußert. $\Delta \nu$ ist die volle Halbwertsbreite in der Verstärkungskurve des YAG-Kristalls und $M$ die Anzahl an halben Wellenlängen der ersten Lasermode (Index 0) innerhalb des Resonators der Länge L. c bezeichnet die Lichtgeschwindigkeit und $\langle$.$\rangle die räumliche$ Mittelung

$$
\langle f(x)\rangle=\kappa \int_{0}^{\infty} e^{-\kappa x} f(x) \mathrm{d} x
$$

mit $\kappa=k L$, was wiederum dem Vorgang des inhomogenen Pumpens Rechnung trägt, da die äußere Pumpe den YAG-Kristall nur bis zu einer charakteristischen Länge 1/K durchsetzt. Weiterhin sei angenommen, dass $K L \gg 1$. Wichtig ist nun die Schätzung der einzelnen Koeffizienten in der Nähe des Fixpunktes. Eine Vereinfachung lässt sich durch die Taylor-Entwicklung des Ausdruckes

$$
\frac{1}{1+\sum_{l} I_{l}^{0} u_{l}(x)} \approx 1-\sum_{l} I_{l}^{0} u_{l}(x)
$$

erzielen. Durch diese Approximation können der nichtlineare Ausdruck für die Kreuzsättigung

$$
\beta_{l j}=\left\langle\frac{u_{l}(x) u_{j}(x)}{1+\sum_{m} I_{m}^{0} u_{m}(x)}\right\rangle
$$

und der Tensor für die Momente zweiter Ordnung

$$
\gamma_{l j k}=\left\langle\frac{u_{l}(x) u_{j}(x) u_{k}(x)}{1+\sum_{m} I_{m}^{0} u_{m}(x)}\right\rangle
$$


in einem ersten Schritt in die Gleichungen

$$
\begin{gathered}
\beta_{l j} \approx\left\langle u_{l}(x) u_{j}(x)\right\rangle-\sum_{m} I_{m}^{0}\left\langle u_{m}(x) u_{l}(x) u_{j}(x)\right\rangle \\
\gamma_{l j k} \approx\left\langle u_{l}(x) u_{j}(x) u_{k}(x)\right\rangle-\sum_{m} I_{m}^{0}\left\langle u_{m}(x) u_{l}(x) u_{j}(x) u_{k}(x)\right\rangle
\end{gathered}
$$

überführt werden. Anschließend sind die jeweiligen Mittelungen über den Bereich des Resonators auszuführen, was an dieser Stelle nur zusammenfassend wiedergegeben sein soll. Man erhält

$$
\begin{gathered}
\left\langle u_{l}\right\rangle \approx g_{l} \\
\left\langle u_{l} u_{j}\right\rangle \approx g_{l} g_{j}\left(1+\frac{K_{l-j}}{2}\right) \\
\left\langle u_{l} u_{j} u_{k}\right\rangle \approx g_{l} g_{j} g_{k}\left(1+\frac{K_{l-j}+K_{l-k}+K_{j-k}}{2}\right) \\
\left\langle u_{l} u_{j} u_{k} u_{m}\right\rangle \approx g_{l} g_{j} g_{k} g_{m}\left(1+\frac{K_{l-j}+K_{l-k}+K_{l-m}+K_{j-k}}{2}\right. \\
\left.+\frac{K_{j-m}+K_{k-m}}{2}+\frac{K_{l+j-k-m}+K_{l-j-k+m}+K_{l-j+k-m}}{8}\right)
\end{gathered}
$$

mit den Koeffizienten

$$
K_{m}=\langle\cos (2 \pi m x)\rangle=\frac{1}{1+\zeta m^{2}}, \quad \zeta=\left(\frac{2 \pi}{\kappa}\right)^{2} .
$$

Aus all diesen Vereinfachungen kann nun schließlich der Tensor $T_{l j k}$ ermittelt werden, der die Momente erster Ordnung mit denen zweiter Ordnung durch

$$
T_{l j k}=\sum_{m} \gamma_{m l j}\left(\beta^{-1}\right)_{m k}
$$

verknüpft. Dabei wurden in den Simulationen die folgenden Parameterwerte verwendet: Resonatorumlaufzeit $\tau_{c} \approx 0.167 \mathrm{~ns}$, Länge des Resonators $L=2.5 \mathrm{~cm}, K=5 \mathrm{~cm}^{-1}$, $\Delta \nu=120 \mathrm{GHz}, \kappa \approx 12.5$.

Ein genauerer Blick auf Gl. (5.5a) zeigt auch die Schwierigkeiten, die mit der Frequenzverdopplung zusammenhängen. Dabei handelt es sich im einzelnen um den Wettkampf zweier konkurrierender Mechanismen zur Erzeugung der frequenzverdoppelten Laserstrahlung. Die Terme $\propto I_{j}^{2}$ entsprechen dem Vorgang der Bildung der Zweiten Harmonischen. Dabei koppeln zwei Photonen derselben Mode zur Erzeugung eines neuen Photons mit der hal- 
5. Kontrolle chaotischer Laserdynamik

ben Wellenlänge. Daneben gibt es den Vorgang der Summenfrequenzbildung, dem die Terme $\propto I_{l} I_{j}$ entsprechen. Im Detail koppeln hier zwei Photonen unterschiedlicher Moden. Beide Mechanismen gehen mit unterschiedlicher Stärke in die Bildung der Gesamtintensität $I=\sum_{j} I_{j}$ ein. Hieraus ergibt sich unter Berücksichtigung der Symmetrie des Modells bezüglich der Moden die grüne (frequenzverdoppelte) Intensität zu

$$
\begin{aligned}
G & \propto \sum_{j=1}^{N} p_{1} g\left(I_{j}-I_{j}^{0}\right) I_{j}-2 p_{2} \sum_{\substack{l=1 \\
l \neq j}}^{N} \mu_{l j}\left(I_{l}-I_{l}^{0}\right) I_{j} \\
& =p_{1} \sum_{j=1}^{N} I_{j}^{2}+4 p_{2} \sum_{\substack{j, l=1 \\
j \neq l}}^{N} \mu_{l j} I_{l} I_{j}-\sum_{j=1}^{N}\left(I_{j}^{0}-I_{l}^{0}\right) I_{j} .
\end{aligned}
$$

Die beiden Proportionalitätsfaktoren $p_{1}$ und $p_{2}$ sind in ihrer Größe unterschiedlich und gewichten somit die Anteile der Zweiten Harmonischen und der Summenfrequenzbildung verschieden stark. Gerade die Summenfrequenzbildung sorgt aber als zusätzlicher nichtlinearer Effekt für eine Destabilisierung und damit für das Auftreten von chaotischen Fluktuationen in der Ausgangsintensität des Lasers. Gl. (5.20) kann analog zu [157] in die Formel

$$
G \propto 8 I_{x} I_{y}+4 \mathrm{~g}\left(I_{x}-I_{y}\right)^{2}-3 \mathrm{~g} \sum_{j=1}^{N} I_{j}^{2}
$$

überführt werden. Danach ist in erster Näherung die Intensität des grünen Laserlichtes proportional zu $8 I_{x} I_{y}$, wobei diese Näherung jedoch nur bei kleinen infraroten Intensitäten $I_{x}, I_{y}$ in den beiden Polarisationsrichtungen $(x, y)$ bzw. kleinen Pumpraten Sinn macht. Mit wachsendem Pumpstrom wird diese Näherung immer stärker verletzt, es müssen dann weitere nichtlineare Terme berücksichtigt werden. Sowohl im Experiment als auch beim Lasermodell stellt sich heraus, dass die beiden infraroten Einzelintensitäten beim Verlassen des Fixpunktes gegenphasig schwingen, d.h. $\delta I_{y}=-q \delta I_{x}$ unter Verwendung des Proportionalitätsfaktors $q$. Damit scheidet die infrarote Gesamtintensität $I_{\mathrm{IR}}=I_{x}+I_{y}$ als Regelgröße aus, da z. B. für $q=1$ ein Verlassen des Fixpunktes anhand der infraroten Gesamtintensität im Gegensatz zu den infraroten Einzelintensitäten erst nach dem Auftreten anharmonischer Terme und damit nur verzögert detektiert werden kann. Ähnliche Betrachtungen in Abhängigkeit von der Position des KTP- und Verdopplerkristalls sind in [157] nachzulesen.

\subsection{Der experimentelle Aufbau}

Das im letzten Abschnitt vorgestellte Lasermodell zeigt in guter Näherung die im Laserexperiment feststellbare Dynamik. Montiert ist das betrachtete Lasersystem auf einem stabilen und schwingungsisolierten optischen Tisch der Firma Newport, wobei der fre- 


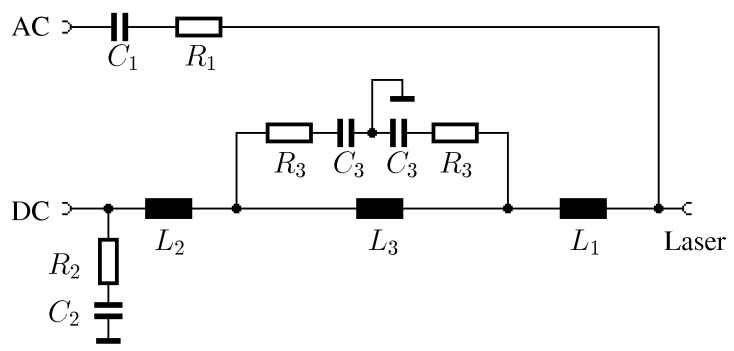

\begin{abstract}
Abb. 5.2: Schematischer Aufbau des verwendeten Bias- T, um auf den von dem Lasercontroller gelieferten Gleichspannungspegel (DC) eine Wechselspannung (AC) aufzumodulieren. Maßnahmen zur Frequenzkompensation wurden bereits intern verfolgt.
\end{abstract}

quenzverdoppelnde Laser $\mathrm{u}$. a. aus einer Pumplaserdiode $(\lambda=808 \mathrm{~nm})$ und einem separat an einem xyz-Tisch aufgehängten zweiten Resonatorspiegel besteht. Vor diesem zweiten Spiegel sind auch der YAG- und der frequenzverdoppelnde KTP-Kristall angebracht. Dieser getrennte Aufbau erlaubt zum einen die genaue Positionierung der Festkörperkristalle vor der Pumplaserdiode und damit eine Optimierung der Ausgangsintensität. Zum anderen bringt diese Aufhängung aber auch Nachteile in Form von Erschütterungsempfindlichkeit. An mehreren Stellen im Strahlengang sind Filter installiert, um die unerwünschte Pumpstrahlung zu eliminieren oder aber die relevante Laserstrahlung in ihrer Intensität zu mindern bzw. zu selektieren.

Der komplette experimentelle Aufbau für die Regelung dieses frequenzverdoppelten Lasers kann dem Schema der Abb. 5.3a entnommen werden. Das gesamte Lasersystem wird durch eine Stromquelle betrieben, die den nötigen Pumpstrom $\mathrm{w}_{0}$ bereitstellt. Das vom Laser ausgesandte Licht mit den Wellenlängen $1064 \mathrm{~nm}$ für die unverdoppelte und $532 \mathrm{~nm}$ für die frequenzverdoppelte Laserstrahlung durchläuft einen frequenzselektiven Strahlteiler, mit dessen Hilfe das grüne (linear polarisierte) Licht aus dem Strahlengang entfernt wird. Das verbleibende nicht frequenzverdoppelte infrarote Licht besteht aus den beiden senkrecht zueinander polarisierten Intensitäten $I_{x}$ und $I_{y}{ }^{3}$. Die AC-gekoppelten Anteile dieser beiden Intensitäten $\left(\tilde{I}_{x}, \tilde{I}_{y}\right)$ oder die grüne Intensität $\tilde{G}$ sind wiederum die Eingangssignale der Regelung, mit deren Hilfe schließlich der Pumpstrom des Lasers moduliert wird. Die Modulation erfolgt über einen sogenannten Bias-T (Modulationspunkt, Picosecond Pulse Labs, PSPL Model 5595-LC), über den AC-Signale auf den bestehenden DC-Pegel der Stromquelle aufmoduliert werden können. Dabei handelt es sich bei dem Bias-T schematisch um einen Hochpass in einem Zweig des Aufbaus, um Wechselspannungssignale auf den Gleichspannungspegel im anderen Zweig zu modulieren (Abb. 5.2). Um die aufmodulierten Wechselspannungssignale vom Laserdiodencontroller zu entkoppeln, befindet sich im DC-Zweig des Bias-T eine Spule, so dass am Ausgang des Bias-T ein Gleichspannungssignal vom Laserdiodencontroller mit aufgesetztem Wechselspannungsanteil vorliegt. Intern sind allerdings noch weitere Maßnahmen zur Frequenzkompensation zu finden.

Im Experiment stehen zunächst drei Parameter zur Verfügung. Mit dem Laserdiodencontroller (Newport, Model 6000) können der Pumpstrom und die Laserdiodentemperatur,

\footnotetext{
${ }^{3}$ Der gesamte Aufbau zur Aufnahme der infraroten Intensitäten ist drehbar um die optische Achse gelagert, damit die Strahlteilung möglichst in Richtung der Eigenpolarisationsrichtungen geschieht.
} 
Abb. 5.3: (a) Experimenteller Aufbau zur Regelung chaotischer Fluktuationen in der Ausgangsintensität (Typ-II) eines grünen $(\lambda=532 \mathrm{~nm})$ intern frequenzverdoppelten Nd:YAG Lasers. Die Regelung moduliert über einen Modulationspunkt (Bias) den Pumpstrom. (b) Der frequenzverdoppelte Laser im Experiment: Rechts im Bild ist die Pumplaserdiode und davor der zweite (separat an einem xyz-Tisch aufgehängte) Resonatorspiegel mit dem Laserresonator $\mathrm{zu}$ sehen (kurze Messingröhre). Innerhalb des Laserresonators sind sowohl der YAG- als auch der KTP-Verdopplerkristall untergebracht. Zum Abschätzen der Größe des Lasers: benachbarte Bohrungen auf dem optischen Tisch besitzen einen Abstand von $2.5 \mathrm{~cm}$. (a)

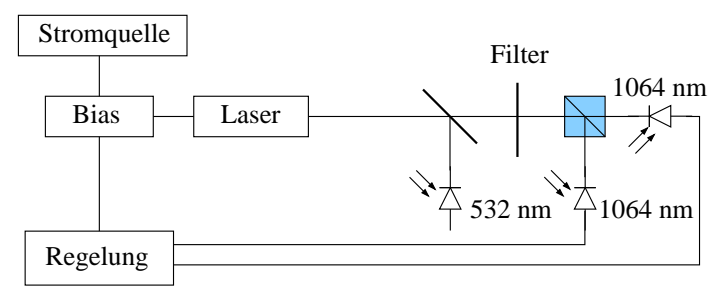

(b)

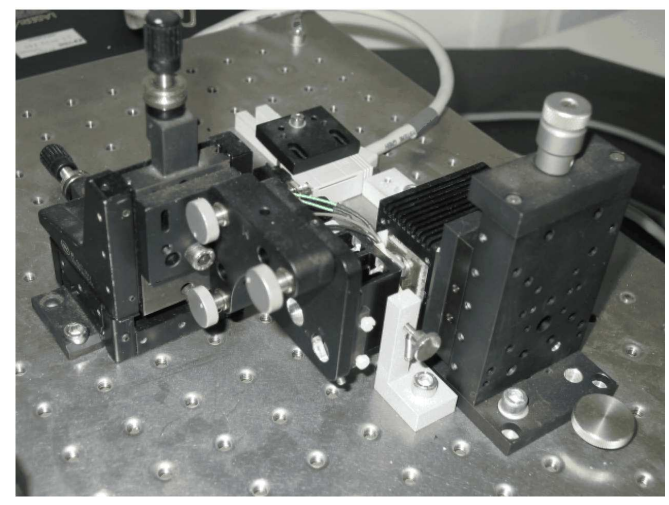

mit dem Temperatursteuergerät (Profile, TED200) die Temperatur des separat aufgehängten Resonatorspiegels verändert werden. Alle drei Parameter haben starken Einfluss auf die Dynamik des Lasers, die zunächst einer eingehenden Untersuchung unterzogen worden ist. Dabei wurde je nach Höhe des Pumpstromes mit dem Grassberger-ProcacciaAlgorithmus eine Korrelationsdimension $D_{2}{ }^{4}$ von $D_{2} \in[3.2,4.6]$ festgestellt. Innerhalb des (offenen) Systems besteht eine durch den Aufbau bedingte Totzeit von $\approx 0.45 \mu \mathrm{s}$, was bei einer Hauptfrequenz von ca. 1.3 MHz einer halben Schwingung entspricht (Abb. 5.4). Diese im Vergleich zur Systemdynamik lange Totzeit erschwert die Regelung auf einen Fixpunkt in erheblichem Maße. Zudem bereitet die systemimmanente Instationarität Schwierigkeiten, die sich im Bereich einiger ms bis Minuten bewegt (bei konstanten Laserparametern, d.h. Pumpstrom, Laserdiodentemperatur, Spiegeltemperatur). Aus diesem Grund sind groß angelegte Rastermessungen an dem Lasersystem nicht sinnvoll, da die Bedingungen für die verschiedenen (Regelungs-) Parameter nicht reproduzierbar sind. Lediglich kurze Messungen des im Vergleich zur Spiegel- und Laserdiodentemperatur schnell veränderlichen Pumpstromes erscheinen sinnvoll und führen zu den Ergebnissen aus Abb. 5.4. Gut sichtbares grünes Licht tritt ab einem Pumpstrom von ca. $700 \mathrm{~mA}$ auf, der entsprechende Fixpunkt geht dann via Hopf-Bifurkation ${ }^{5}$ in einen periodischen Orbit über, der auf dem

\footnotetext{
${ }^{4}$ Das Korrelationsintegral $C(\varepsilon)=\lim _{N \rightarrow \infty} N^{-1}(N-1)^{-1} \sum_{j, l=1}^{N} \Theta(\varepsilon-\|\mathbf{x}(j)-\mathbf{x}(l)\|), \quad \mathbf{x}(j) \in \mathbb{R}^{m}$, $j \neq l$ ist die mittlere Wahrscheinlichkeit dafür, dass Zustände zu zwei unterschiedlichen Zeiten $j, l$ aufgrund der Dynamik weniger als $\varepsilon$ voneinander entfernt sind. $\mathbf{x}(j)$ ist der gemessene (bzw. rekonstruierte) Zustandsvektor zur Zeit $j$, von denen zur Analyse nur $N$ herangezogen werden. $\|\cdot\|$ bezeichnet eine Norm und $\Theta(\cdot)$ die Heaviside-Funktion. Die Korrelationsdimension berechnet sich zu $D_{2}=\lim _{\varepsilon \rightarrow 0} \ln (C) / \ln (\varepsilon)$.

${ }^{5}$ Eine (lokale) Bifurkation ist eine qualitative Änderung der Dynamik, wobei im Zuge der Hopf-
} 


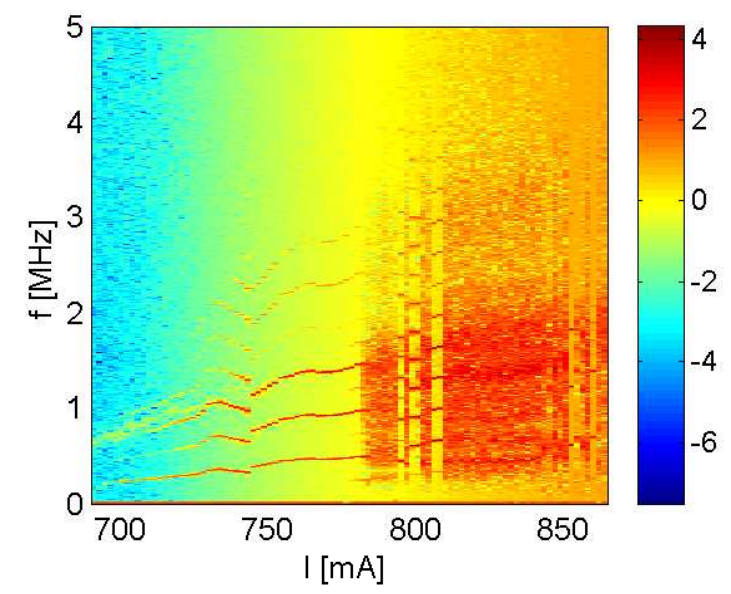

Abb. 5.4: Bifurkationsdiagramm des verwendeten frequenzverdoppelten Lasers. Die in Abhängigkeit vom Pumpstrom auftretenden Frequenzen sind logarithmisch farbkodiert. Die Laserdioden- und Spiegeltemperatur wurden konstant gehalten. Der systemeigene Fixpunkt geht via Hopf-Bifurkation in einen Periode-3Orbit über, wobei sich die auftretenden Frequenzen nach Durchlaufen einiger periodischer Fenster auf bis zu $1.8 \mathrm{MHz}$ innerhalb des Chaosbereichs erhöhen.

Weg ins Chaos von teilweise höherperiodischen Orbits abgelöst wird. Die chaotische Dynamik der beiden infraroten Einzelintensitäten ist dabei unterschiedlich, während beide Intensitäten bei Annäherung nahezu phasengleich (stabile Richtung) bzw. bei Verlassen des Fixpunktes gegenphasig schwingen (instabile Richtung). Ist die Dynamik allerdings auf einen Fixpunkt relaxiert, sind sowohl die beiden infraroten als auch die grüne Intensität konstant.

\subsection{Laser-Stabilisierung}

Für technische Anwendungen ist gerade dieser Zustand einer konstanten Ausgangsintensität für frequenzverdoppelte Laser notwendig, was eine große Herausforderung für alle Regelungsmethoden darstellt. Unglücklicherweise verschlimmert sich dieses Problem für kompakte Laser, die eine starke Tendenz zu Instabilitäten besitzen und zudem durch das kompakte Design chaotischer Dynamik im MHz-Bereich unterliegen. Und so sind seit den ersten Untersuchungen des green problems bei intern frequenzverdoppelten Nd:YAG Lasern durch Baer [16] viele Anstrengungen unternommen worden, um diese Art der dynamischen Instabilität zu beseitigen oder zumindest zu reduzieren. Dabei bieten sich verschiedene Möglichkeiten an, angefangen mit optischen Korrekturen. So kann z. B. ein $\lambda / 4$ Plättchen innerhalb des Laserresonators eingefügt werden [68,104], wodurch bei richtiger Wahl des Winkels zwischen den schnellen Achsen der beiden Kristalle die Summenfrequenzbildung unterdrückt und nur die Erzeugung von grünem Laserlicht durch die Zweite Harmonische ermöglicht wird. Die Moden sind dann ungekoppelt und die Ausgangsintensität dementsprechend konstant, was aber auch durch eine bessere Ausrichtung des KTP-Kristalls in Bezug auf den YAG-Kristall hervorgerufen werden kann [30,67]. Eine

Bifurkation ein Fixpunkt des betrachteten dynamischen Systems seine Stabilität verliert. Stattdessen entsteht ein periodischer Orbit. Dieser sogenannte Grenzzyklus ist stabil, wenn der Realteil der Eigenwerte null ist (superkritisch) oder instabil, wenn die entsprechenden Realteile $>0$ sind (subkritisch). 
weitere Möglichkeit besteht in der Verwendung eines L-förmigen Resonators [151]. Darüber hinaus wurde vorgeschlagen, den Laserresonator entsprechend lang zu gestalten, um eine Energieverteilung auf eine größere Anzahl von Moden zu erreichen. Das wäre natürlich stabiler, allerdings ist dieser Weg im Zeitalter der Miniaturisierung nicht unbedingt erwünscht. So kann auch festgestellt werden, dass optische Eingriffe zwar bei den Firmen sehr verbreitet sind, aber unter anderen Gesichtspunkten nicht immer das Mittel der Wahl darstellen. Jede zusätzliche optische Komponente ist mit weiteren Kosten und Justieraufwand verbunden, den Hersteller nur ungern auf sich nehmen möchten.

Von daher sind Methoden, die zusätzlich zu den bereits bestehenden Lasersystemen anwendbar sind, bevorzugt. Unter diesem Gesichtspunkt betrachteten viele Autoren die Dynamik dieser Laser mit dem Ziel, Chaos-Kontrollmethoden bei einem geeigneten Systemparameter, z. B. dem Pumpstrom zu applizieren, um ihre Dynamik zu bändigen [4$6,33,36,49,50,119,120,131,157$. Roy et al. [131] waren schon 1992 erfolgreich damit, experimentell instabile periodische Orbits (UPOs) mit Hilfe von Occasional Proportional Feedback (OPF, Details im Anhang ab Seite 118) zu stabilisieren. Gills et al. [50] haben daraufhin diesen Ansatz mit einer Tracking-Prozedur kombiniert und damit auch die Stabilitätsgebiete erweitern können. Die erfolgreiche OPF-Kontrolle von UPOs wurde dann auch bald von Colet et al. [36] in Simulationen gezeigt. Dabei kam das MultimodenLasermodell von Carr und Schwartz [33] zum Einsatz, wobei schließlich auch Fixpunkte mit einer abgewandelten Form von OPF stabilisiert werden konnten. Alternativ hierzu wurde in Simulationen [119,120] konventionelle PD-Kontrolle (2.1) zur Fixpunktstabilisierung verfolgt. Als Eingangssignale für die Regelung dienten dabei die beiden senkrecht zueinander polarisierten infraroten, nicht frequenzverdoppelten Intensitäten eines frequenzverdoppelten Nd:YAG Lasers. Experimentell wurden diese Untersuchungen von Schenck zu Schweinsberg und Dressler [157] an einem Lasersystem mit Intensitätsschwankungen von ungefähr $30 \mathrm{kHz}$ durchgeführt.

Es bleibt allerdings festzuhalten, dass alle diese Regelungsmethoden bei mittleren und höheren Pumpraten, bei denen das sogenannte Typ-II-Chaos auftritt [1,84], versagten. Dabei sind Moden in unterschiedlichen Polarisationsrichtungen aktiv, was beim einfacheren Typ-I-Chaos nicht der Fall ist. In den meisten Fällen ist Typ-II-Chaos höherdimensionaler als Typ-I-Chaos und damit auch schwieriger zu eliminieren. Für Anwendungen werden wiederum Laser, die über Typ-II-Chaos verfügen, erwünscht sein, da bei ihnen zum einen wegen des Mehrmodenbetriebes intensivere frequenzverdoppelte Strahlung entsteht im Vergleich zum Monomodenbetrieb und zum anderen in der Praxis aus Stabilitätsgründen des Resonators (z. B. hemisphärischer Aufbau) das Auftreten von mehreren unterschiedlich zueinander polarisierten Moden nur schwer vermieden werden kann.

Eigene Experimente bestätigten die generelle Beobachtung, dass es nahezu unmöglich ist, einen kompakten intern frequenzverdoppelten Nd:YAG Laser (Typ-II Chaos, Hauptfrequenzen zwischen $1 \mathrm{MHz}$ und $1.8 \mathrm{MHz}$, Systemtotzeit $\approx 0.45 \mu \mathrm{s}$, Leistungen $\mathrm{im} \mathrm{mW}-$ Bereich) mit einem konventionellen P-, PD- oder TDAS-Regler (2.10) bzw. einem einpoligen Hochpassfilter (2.13) als Grenzfall von ETDAS (2.11) zu stabilisieren. Motiviert durch 
diese schweren Beschränkungen habe ich MDFC und NFF entwickelt und experimentell sowie in Simulationen angewendet, um die auftretenden chaotischen Intensitätschwankungen erfolgreich auszuregeln.

\subsection{Regelung des Lasers in der Praxis}

Als Eingangsgrößen für die Regelung dienen dazu wahlweise die beiden AC-gekoppelten infraroten Intensitäten $\tilde{I}_{x}$ und $\tilde{I}_{y}(1064 \mathrm{~nm})$ oder die AC-gekoppelte grüne Intensität $\tilde{G}$ mit der Wellenlänge $532 \mathrm{~nm}$. Diese Signale werden mittels analoger Elektronik im Falle von MDFC verzögert (All-Pass-Filter, die als Bessel-Filter betrieben werden) oder mit NFF gefiltert und anschließend wieder über das Bias-T (Modulationspunkt) auf den Pumpstrom zurückgekoppelt. Im Detail verläuft die Pumpstrommodulation mittels MDFC und der infraroten Intensitäten gemäß

$$
\Delta \mathrm{w}(t)=a_{x} \tilde{I}_{x}\left(t-\tau_{x}\right)-b_{x} \tilde{I}_{x}(t)+a_{y} \tilde{I}_{y}\left(t-\tau_{y}\right)-b_{y} \tilde{I}_{y}(t)
$$

bzw. unter Verwendung der grünen Intensität als

$$
\Delta \mathrm{w}(t)=a_{x} \tilde{G}\left(t-\tau_{x}\right)-b_{x} \tilde{G}(t)+a_{y} \tilde{G}\left(t-\tau_{y}\right)-b_{y} \tilde{G}(t)
$$

Die sechs Parameter $\left(a_{x}, b_{x}, a_{y}, b_{y}, \tau_{x}, \tau_{y}\right)$ werden experimentell eingestellt, um eine Stabi-

(a)

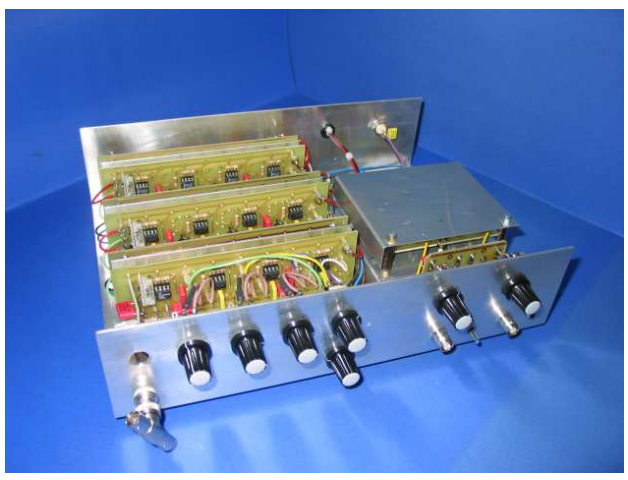

(b)

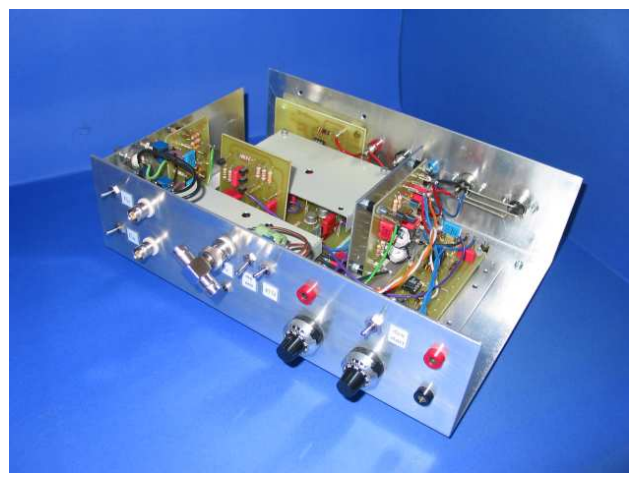

Abb. 5.5: MDFC für das Laserexperiment. (a) Die Verzögerungszeiten für die beiden infraroten Intensitäten wurden mit einer elektronischen Verzögerungskette, die aus Allpassfiltern besteht, realisiert. Die gewichtete Differenzbildung mit dem unverzögerten Eingangssignal erfolgt bereits intern. (b) Ausgangsverstärker für das Regelsignal.

lisierung des Fixpunktes herbeizuführen. In beiden Fällen sind $\tau_{1} \approx 0.6 \mu$ s und $\tau_{2} \approx 2.8 \mu \mathrm{s}$ typische Werte für die angewendeten Verzögerungszeiten. Abb. 5.6a zeigt den Beginn des 
Regelprozesses bei einer Pumprate, die dreimal über der Laserschwelle lag. Nach kurzen Transienten werden die für das ungeregelte System auftretenden Fluktuationen in der Ausgangsintensität auf Rauschniveau reduziert, wobei das kurze nochmalige Entfernen vom Fixpunkt von der mechanischen Ausführung des Schalters bei Aktivierung der

(a)

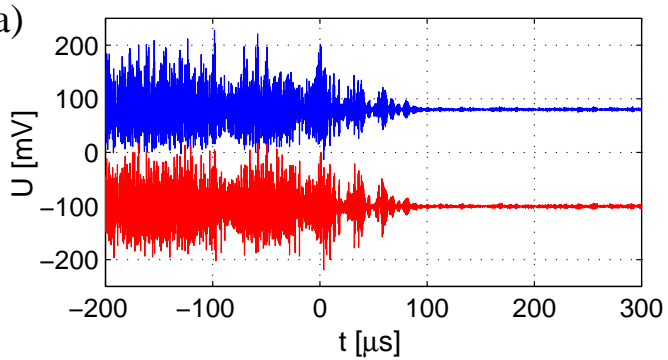

(b)

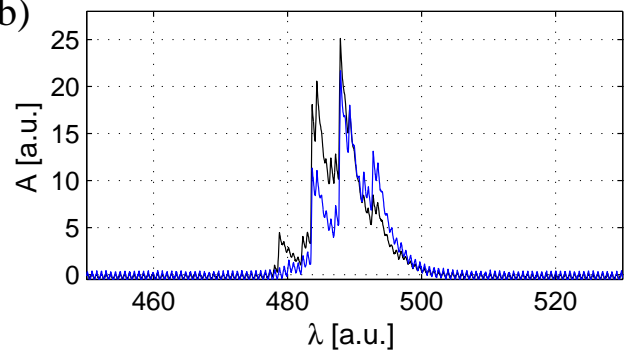

Abb. 5.6: Erfolgreiche Stabilisierung der Intensität des frequenzverdoppelten Nd:YAG Lasers unter Anwendung von MDFC (5.22). (a) Dargestellt sind die beiden (AC-gekoppelten) senkrecht zueinander polarisierten, nicht frequenzverdoppelten infraroten Intensitäten, die auch gleichzeitig die Eingangssignale für die Regelung sind und damit der Modulation des Pumpstromes dienen. Das Regelsignal wurde zur Zeit $t=0$ aktiviert. (b) Veränderung der Modenstruktur vor (schwarz) und nach (blau) der Aktivierung einer nicht optimal eingestellten Regelung, wodurch sich die Zahl der aktiven Moden von vier auf drei verringert. Die Energie wird neben Verlusten auf die anderen Moden verteilt.

Regelung (Abb. 5.5) herrührt (Kontaktprellen). Üblicherweise sind bei diesem Laser bei abgeschalteter Regelung zwischen drei und fünf Moden aktiv - je nachdem, wie groß die Pumprate ist. Versuche, den Laser bei diesen Bedingungen mit PID-Kontrolle (2.1) oder TDAS (2.10) etc. zu stabilisieren, sind fehlgeschlagen. Bei schlecht eingestellten Kontrollparametern wird die Zahl der aktiven Lasermoden nach Aktivierung der Regelung von vier auf drei reduziert (Abb. 5.6), wobei die Energie in diesem Fall hauptsächlich auf die anderen Moden verteilt wird. Bei geeigneter Wahl der Regelungsparameter bleibt aber die Anzahl der Moden auch nach dem Zuschalten der Regelung erhalten, die auftretenden Verluste sind äußerst gering.

Für NFF mit zwei Kerbfiltern (3.2), (3.3) ist die Pumpstrommodulation durch die Differenz der gefilterten Ausgangssignale $V_{\text {out }}^{(1)}, V_{\text {out }}^{(2)}$ und der Filtereingangssignale $V_{\text {in }}^{(1)}, V_{\text {in }}^{(2)}$ entsprechend

$$
\Delta \mathrm{w}(t)=k_{1}\left[V_{\text {in }}^{(1)}-V_{\text {out }}^{(1)}\right]+k_{2}\left[V_{\text {in }}^{(2)}-V_{\text {out }}^{(2)}\right]
$$

gegeben. Hierfür stehen entweder die AC-gekoppelten Komponenten der infraroten Intensitäten $\tilde{I}_{x}, \tilde{I}_{y}$ oder die grüne Intensität $\tilde{G}$ zur Verfügung, um eine nicht-invasive Regelung zu gewährleisten [8]. Die Einstellung der Parameter ist bei geeignet gewählter Filtergüte $Q$ für NFF relativ einfach, wobei die experimentelle Wirkungsweise durch eine zusätzliche Modulation der Resonanzfrequenz eines der beiden Kerbfilter weiter verbes- 


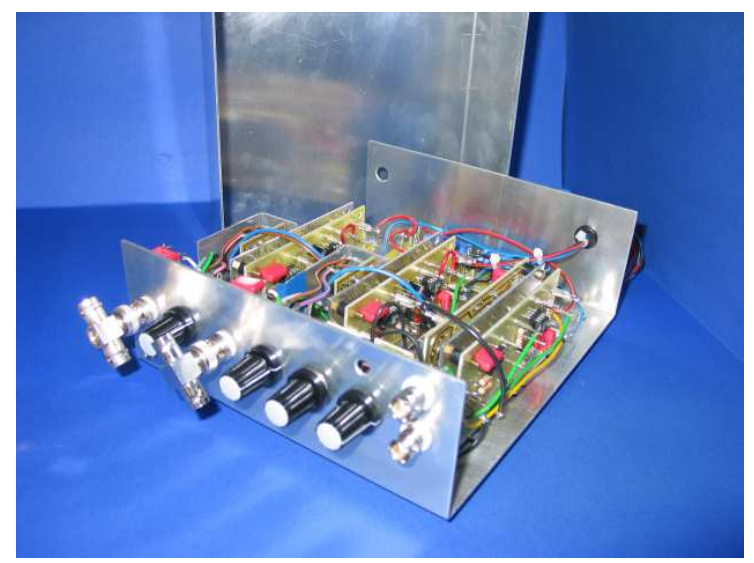

Abb. 5.7: NFF für das Laserexperiment. Das gezeigte Gerät enthält bereits zwei von außen mit einer Steuerspannung modulierbare Wien-Brücken. Die Güte der Filter wird durch den internen Aufbau festgelegt und kann von außen im Gegensatz zur Filter-Resonanzfrequenz nicht geändert werden. Die Filter-Ausgangssignale werden anschließend mit der Endstufe aus Abb. 5.5b verstärkt und modulieren via Bias-T den Pumpstrom des Lasers.

sert werden kann (siehe Abschnitt 3.1). Als Eingangssignal hat sich für diesen Zweck die AC-gekoppelte (chaotische) grüne Intensität als geeignet herausgestellt, die entsprechend verstärkt zur Regelung einer der Wien-Brücken herangezogen wird. Hiermit ist es auch möglich den Einfluss der Instationarität in einem bestimmten Parameterregime zu minimieren und einen stabilen Laserbetrieb über eine längere Zeitspanne zu ermöglichen. Ein Beispiel für solch eine längere Fixpunktstabilisierung ist der Abb. $5.8 \mathrm{zu}$ entnehmen, die bei abgeschalteter Regelung chaotische Laserdynamik zeigt. Dargestellt sind die dyna-

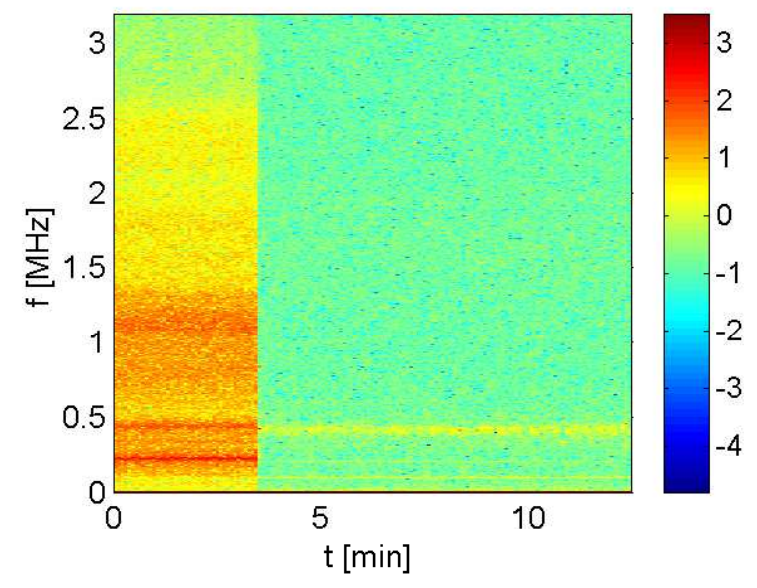

\begin{abstract}
Abb. 5.8: Fixpunktstabilisierung mit zwei Kerbfiltern (NFF), wobei ein WienFilter (3.2) zusätzlich durch die grüne Intensität $\tilde{G}(t)$ in seiner Resonanzfrequenz moduliert wird (3.5). Nach Aktivierung der Regelung zur Zeit $t \approx 4 \mathrm{~min}$ werden die Regelparameter nicht mehr verändert. Anhand des spektralen Bifurkationsdiagramms lässt sich eine längerfristige Stabilisierung der Ausgangsintensität trotz Instationarität erkennen.
\end{abstract}

mischen Zusammenhänge in Form eines zeitlich spektralen Bifurkationsdiagramms, das die Anteile der Einzelfrequenzen der auch als Eingangssignal für die Filter fungierenden grünen Intensität $\tilde{G}$ farbkodiert darstellt. Unter diesem Gesichtspunkt ist zu erkennen, dass nach dem Einschalten der Regelung für $t>4$ min die chaotische Laserdynamik auf einen Fixpunkt reduziert wird und somit trotz Instationarität einen stabilen Laserbetrieb bei unveränderter Parameterwahl ermöglicht. Ein ähnliches Vorgehen zur Minimierung der Auswirkungen der Instationarität habe ich auch für MDFC experimentell getestet, indem die Verzögerungszeiten entsprechend chaotisch moduliert wurden. Weiterhin hat 
sich gezeigt, dass eine periodische Modulation der Resonanzfrequenz der Wien-Brücken oder der Verzögerungszeiten von MDFC nicht zum erwünschten Erfolg führt, sondern im Gegenteil - die Dynamik in den allermeisten Fällen komplizierter gestaltet.

\subsection{Regelung des Lasers in der Simulation}

Der experimentelle Erfolg von MDFC [4-6] und NFF [8] bei der Stabilisierung des kompakten, intern frequenzverdoppelten Nd:YAG Lasers kann auch anhand des Lasermodells aus Gl. (5.5a,b) bestätigt werden [9]. Die Anzahl der aktiven Lasermoden wird genau wie im Experiment durch den Pumpstrom $w_{0}$ vorgewählt. Im folgenden werde ich mich auf den Fall des Dreimodenbetriebes beschränken, der auch für die vorliegende experimentelle Situation besondere Bedeutung hat. Für das Modell bedeutet dies, dass die Pumprate im Bereich $\mathrm{w}_{0} \in[1.153,1.519]$ liegt und die damit verbundene Auswirkung auf die Intensitäten $I_{-1}, I_{0}$ und $I_{1}$ der Moden untersucht wird. Nur in diesem Bereich liefert Gl. (5.5a) für drei aktive Lasermoden physikalisch sinnvolle Lösungen mit $I_{j}>0$. Es ist zugleich der einfachste Fall der Modenkonfiguration, bei dem neben (quasi-)periodischen Orbits und instabilen Fixpunkten auch Chaos auftreten kann. Chaotische Dynamik ist allerdings nicht auf den Dreimodenfall beschränkt, sondern auch für höhere Pumpströme feststellbar, bei denen noch mehr Moden aktiv sind. Aufgrund des Multimodenbetriebes zeigt der Laser (nicht frequenzverdoppelte) infrarote Laserstrahlung in zwei zueinander senkrecht polarisierten Richtungen $(x, y)$. Die infrarote Gesamtintensität ist zur Regelung dieser Art von Lasern nicht geeignet, da beim Verlassen des Fixpunktes die beiden infraroten Intensitäten nahezu gegenphasig schwingen. Folglich zeigt die infrarote Gesamtintensität $I_{\mathrm{IR}}$, die die Summe der beiden Einzelintensitäten darstellt, kein Verlassen des Fixpunktes an. Die Regelung wird erst aktiviert, wenn die Dynamik sich schon weit von dem eigentlichen Fixpunkt entfernt hat und $I_{\text {IR }}$ aufgrund von Nichtlinearitäten der Einzelsignale von Null verschieden ist.

Um den System-Fixpunkt zu stabilisieren, wird MDFC [5, 6] gemäß Gl. (5.22) angewendet. Eingangssignale für die Rückkopplungsschleife sind die beiden AC-gekoppelten infraroten Intensitäten $\tilde{I}_{x}=\tilde{I}_{-1}+\tilde{I}_{1}$ und $\tilde{I}_{y}=\tilde{I}_{0}$. Die Abb. 5.9 zeigt ein Beispiel einer erfolgreichen Fixpunktstabilisierung in der Simulation mit drei aktiven Moden $I_{-1}, I_{0}$ und $I_{1}$. Da das Lasermodell relativ hochdimensional und auch teilweise sehr aufwändig ist, soll an dieser Stelle auf ausgedehnte Stabilitätsanalysen vergleichbar zu denen aus dem zweiten Kapitel verzichtet werden. Vielmehr wird sich auf eine nummerische Integration der Zustandsgleichungen des Lasers für drei aktive Moden beschränkt. Zum Einsatz kommt dabei ein Runge-Kutta-Löser 4. Ordnung mit konstanter Schrittweite. Abb. 5.10 illustriert die auf diese Art und Weise berechneten ausgedehnten Stabilitätsgebiete für MDFC aus Gl. (5.22) in der Projektion von Teilen des Parameterraumes, der in diesem Fall durch die Pumprate $\mathrm{w}_{0}$ und jeweils zwei Verstärkungsfaktoren aus Gl. (5.22) gegeben ist. Die beiden anderen Verstärkungsfaktoren sowie die beiden Verzögerungszeiten $\tau_{x}=0.332 \mathrm{~ms}$ und $\tau_{y}=0.1 \mathrm{~ms}$ 


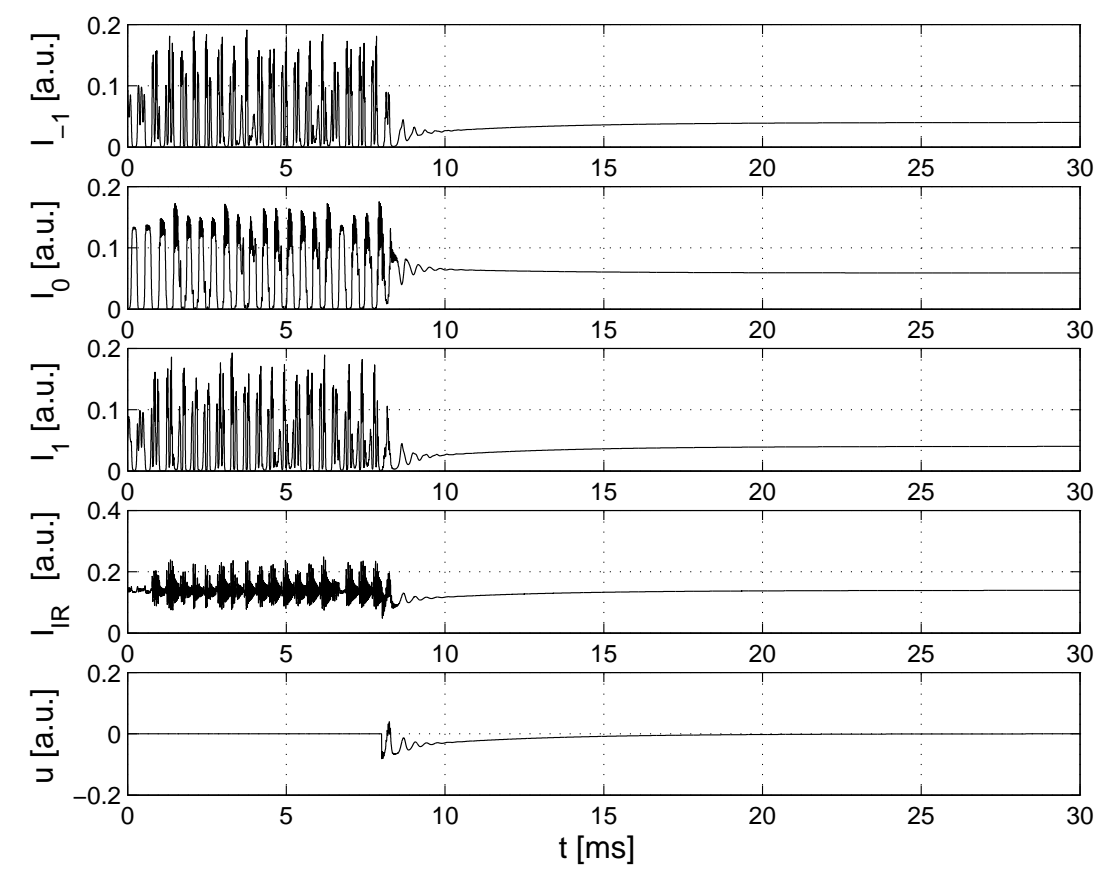

Abb. 5.9: Fixpunktstabilisierung mit MDFC (5.22) und den zwei Verzögerungszeiten $\tau_{x}=0.332 \mathrm{~ms}$ und $\tau_{y}=0.1 \mathrm{~ms}$. Die eingestellte Pumprate entspricht $\mathrm{w}_{0}=1.25$, die Verstärkungsfaktoren sind zu $a_{x}=0.8, b_{x}=0.2, a_{y}=0.1, b_{y}=0.21$ gewählt. Nach dem Aktivieren der Regelung zur Zeit $t=8 \mathrm{~ms}$ relaxiert die Systemdynamik nach kurzen Transienten auf den systemeigenen Fixpunkt. $I_{j}$ bezeichnet die Intensität der drei aktiven Lasermoden $j, I_{-1}$ und $I_{1}$ sind parallel zueinander und senkrecht zu $I_{0}$ polarisiert. $I_{\mathrm{IR}}$ steht für die infrarote Gesamtintensität und $u$ für das angewendete Kontrollsignal (5.22). Eingangsgrößen für die Regelung sind die beiden AC-gekoppelten infraroten Intensitäten $\tilde{I}_{x}=\tilde{I}_{-1}+\tilde{I}_{1}$ und $\tilde{I}_{y}=\tilde{I}_{0}$.

bleiben hierbei jeweils unverändert. Anhand der Abb. 5.10 erkennt man, dass eine Stabilisierung des Fixpunktes praktisch im kompletten Bereich des Dreimodenbetriebes mit Pumpraten $\mathrm{w}_{0}$ zwischen 1.17 und 1.5 möglich ist. Die kompakte Gestalt der Stabilitätsregion offenbart eine stabile Regelung durch MDFC mit einer nicht zu speziellen Wahl der Parameter. Im Gegensatz zu der Stabilisierung mit einem PD-Regler [120] gibt es in diesem Fall keine Subintervalle in dem Stabilitätsgebiet, wo keine Stabilisierung möglich ist. Zieht man die Untersuchungen der anderen dynamischen Systeme [6] sowie die Resultate aus Kapitel 2 in Betracht, so lässt sich vermuten, dass die Stabiltätsgebiete nach dem Zuschalten weiterer geeignet gewählter Verzögerungszeiten zusätzlich ausgedehnt werden. Mit Hilfe der Stabilitätsdiagramme erkennt man zudem, dass eine Stabilisierung praktisch nur bei asymmetrisch gewählten Verstärkungsfaktoren $a_{x} \neq b_{x}$ und $a_{y} \neq b_{y}$ möglich ist. Im Gegensatz zu der ursprünglichen Pyragas-Regelung (2.10), die für den betrachteten Dreimodenfall keine Fixpunktstabilisierung ermöglicht, ist eine asymmetrische Wahl der Vorfaktoren in der Tat möglich, da für die Stabilisierung des Fixpunktes nur die AC- 
(a)

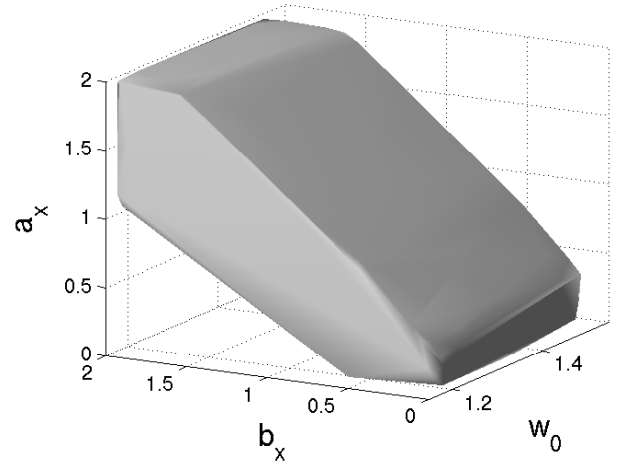

(c)

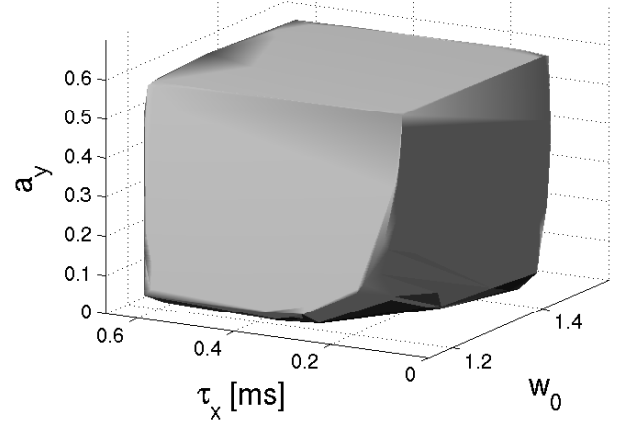

(b)

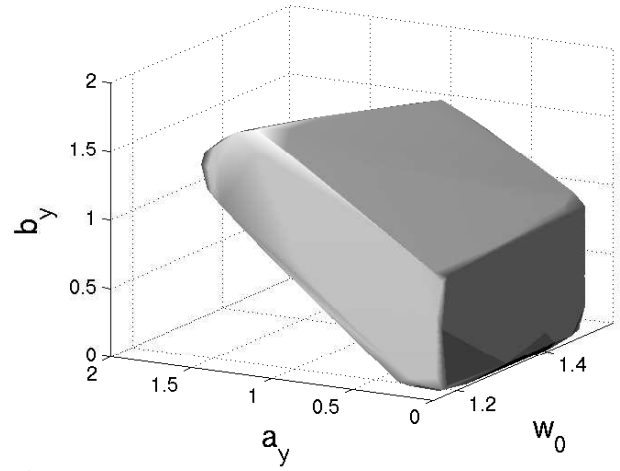

(d)

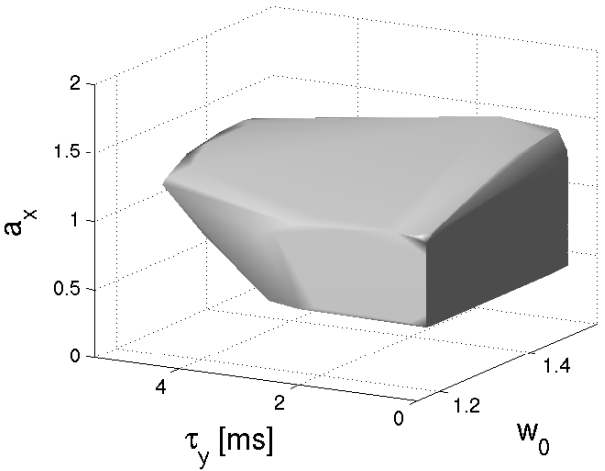

Abb. 5.10: Stabilitätsdiagramme für MDFC (5.22), wobei die Parameter, die zu einer erfolgreichen Fixpunktstabilisierung führen, grau gekennzeichnet sind. Variiert wurde sowohl die Pumprate $\mathrm{w}_{0}$ im Bereich des Dreimodenbetriebes als auch je zwei der Verstärkungsfaktoren. Die beiden Verzögerungszeiten bleiben währenddessen fest auf $\tau_{x}=0.332 \mathrm{~ms}$ und $\tau_{y}=0.1 \mathrm{~ms}$ eingestellt, die jeweils anderen Verstärkungsfaktoren sind zu (a) zweite Verzögerungskette: $a_{y}=0.23, b_{y}=0.51$, (b) erste Verzögerungskette: $a_{x}=1.09, b_{x}=0.63$ gewählt. (c),(d) Variiert wird jeweils eine Verzögerungszeit und ein Vorfaktor, alle anderen Parameter blieben fest eingestellt. (c) $a_{x}=1.09, b_{x}=0.63, b_{y}=0.51, \tau_{y}=0.1 \mathrm{~ms},(\mathrm{~d}) b_{x}=0.63, b_{y}=0.51, a_{y}=0.23$, $\tau_{x}=0.332 \mathrm{~ms}$. In allen Fällen offenbart die Projektion des Parameterraumes ausgedehnte Stabilitätsgebiete.

Signale $\tilde{I}_{x}, \tilde{I}_{y}$ oder $\tilde{G}$ verwendet werden. Bei Erreichen des Fixpunktes verschwindet das Regelsignal automatisch - ungeachtet der Vorfaktoren vor den einzelnen Termen. D. h. das Regelsignal muss keine weiteren Randbedingungen erfüllen, um insgesamt als nichtinvasive Kontrolle [6] zu fungieren. Die Tatsache, dass asymmetrische Vorfaktoren nicht nur erlaubt, sondern für die Stabilisierung geradezu notwendig sind, wurde auch experimentell bestätigt [5]. Demnach ist asymmetrisches MDFC symmetrischem MDFC und auch (E)TDAS überlegen, was anhand des in Kap. 2 und Kap. 4 behandelten Einflusses der Verstärkungsfaktoren auf die Übertragungsfunktion der Regelung verständlich ist. Dabei 


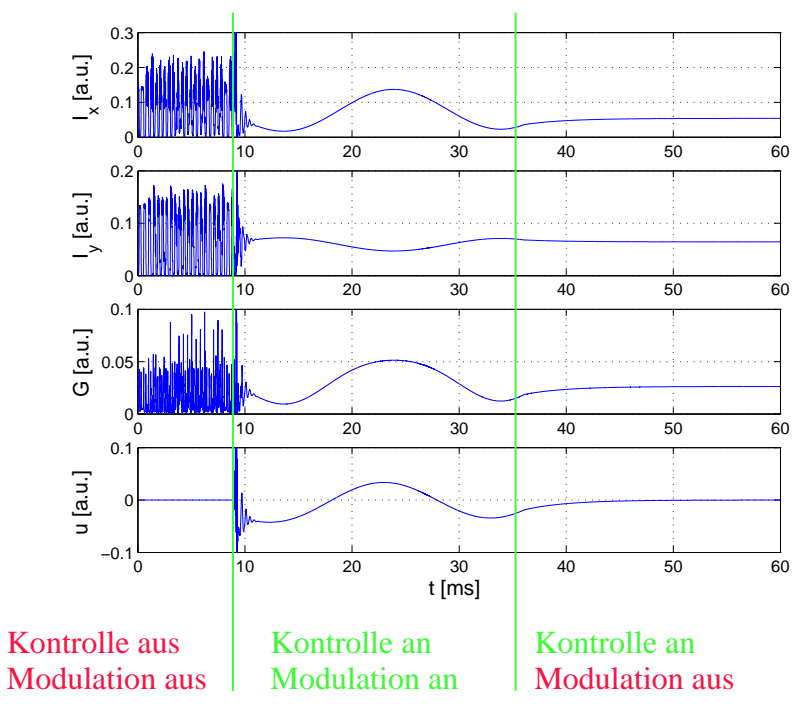

Abb. 5.11: Stabilisierung der Laserdynamik bei gleichzeitiger langsamer Modulation des Pumpstromes $\mathrm{w}(t)=\mathrm{w}_{0}+$ $\Delta \mathrm{w}(t)=1.25+0.029 \sin (\omega t)$ mit $\omega=$ $2 \pi / 20 \mathrm{~ms}$ und festen Kontrollparametern. Die Werte entsprechen denen aus Abb. 5.9, Modulation und Regelung werden beide gleichzeitig für $t=8 \mathrm{~ms}$ eingeschaltet. $G$ bezeichnet die Intensität des generierten grünen Laserlichtes gemäß $G \propto 8 I_{x} I_{y}+4 \mathrm{~g}\left(I_{x}-I_{y}\right)^{2}-$ $3 \mathrm{~g} \sum_{i=-1}^{1} I_{i}^{2}$ mit $\mathrm{g}=0.1$. $u$ entspricht dem angewendeten Regelsignal (5.22), $I_{x}$ und $I_{y}$ den infraroten Intensitäten in $x$ - und $y$-Richtung.

ist beachtenswert, dass eine Veränderung der Verzögerungszeit einer Kette hauptsächlich durch den nichtverzögerten Teil der anderen Verzögerungskette kompensiert werden muss. Dieses Ergebnis hat sich ebenfalls experimentell bestätigt.

Für technische Anwendungen wie holografische Displays ist die Möglichkeit einer weiteren Modulation eines stabilen Lasers essentiell, da die Übertragung von Bildinhalten durch eine Hell-Dunkeltastung der einzelnen farbigen Laser vor sich gehen soll. Dafür darf der Laser natürlich nicht seine Stabilität verlieren. Diese Modulation der Helligkeit des Lasers wird auf sehr viel langsameren Zeitskalen vonstatten gehen als die eigentliche (chaotische) Laserdynamik. Abb 5.11 zeigt eine Simulation mit einer solchen zusätzlichen sinusförmigen Modulation, die mit einer Frequenz von $\approx 50 \mathrm{~Hz}$ auf den Pumpstrom des Lasers wirkt. Aktivierung von MDFC zur Zeit $t=8 \mathrm{~ms}$ führt einerseits zu einer Unterdrückung der chaotischen Fluktuationen in der Ausgangsintensität des Lasers für die beiden infraroten $\left(I_{x}\right.$ und $\left.I_{y}\right)$ und die grüne Intensität $(G)$ und andererseits zu sinusförmigen Oszillationen entsprechend der äußeren Modulation. Die verwendeten Regelparameter lauten $a_{x}=0.8$, $b_{x}=0.2, a_{y}=0.1, b_{y}=0.21, \tau_{x}=0.332 \mathrm{~ms}$ und $\tau_{y}=0.1 \mathrm{~ms}$. Der Modulationseffekt kommt bei der infraroten Intensität $I_{x}$ stärker zum Vorschein als bei $I_{y}$, da $I_{x}=I_{-1}+I_{1}$ aus zwei Moden besteht, die beide moduliert werden im Gegensatz zu $I_{y}=I_{0}$, bestehend aus nur einer modulierten Mode. Anhand des Regelsignals erkennt man, dass es sich hierbei zunächst um ein Beispiel einer invasiven Regelung handelt, da $u(t) \neq 0$. Bei Erreichen des Fixpunktes (d.h. nach Abschalten der zusätzlichen sinusförmigen Modulation) ist dann allerdings mit $u(t)=0$ eine nichtinvasive Regelung gewährleistet.

Neben MDFC war auch NFF geeignet, die Dynamik dieses frequenzverdoppelten Lasers auf einen Fixpunkt zu reduzieren - sowohl im Modell als auch in der Praxis [8]. Eingangssignale $V_{\text {in }}^{(1)}, V_{\text {in }}^{(2)}$ für die Rückkopplung der Gl. (5.24) sind wiederum die beiden nicht frequenzverdoppelten infraroten Intensitäten $\tilde{I}_{x}=\tilde{I}_{-1}+\tilde{I}_{1}$ und $I_{y}=I_{0}$. Abb. 5.12 

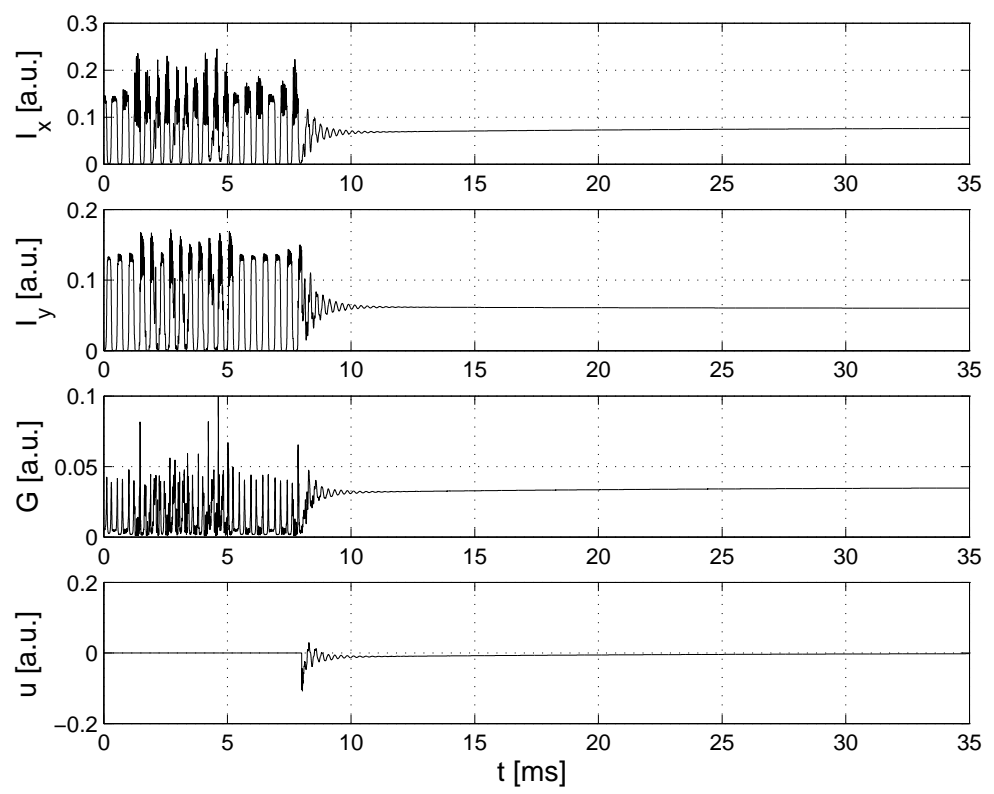

\begin{abstract}
Abb. 5.12: Simulation der Fixpunktstabilisierung eines frequenzverdoppelten Lasers durch die Anwendung von zwei Kerbfiltern (3.2), (3.3). $I_{x}$ und $I_{y}$ bezeichnen die infraroten Intensitäten, deren AC-Anteile als Eingangsgrößen für die Regelung fungieren. $G$ ist die Intensität der frequenzverdoppelten grünen Laserstrahlung, $u(t)$ bezeichnet das Regelsignal (5.24). Der konstante Teil der Pumprate sei $\mathrm{zu}_{0}=1.247$ mit den Kontrollparametern $k_{1}=0.9, k_{2}=1.7, R_{1}=3590 \Omega$, $R_{2}=420 \Omega, C_{1}=C_{2}=25 \mathrm{mF}$ und $Q_{1}=Q_{2}=0.5$ gewählt. Die Regelung wird für $t=8 \mathrm{~ms}$ aktiviert und stabilisiert die Ausgangsintensität des Lasers relativ schnell, wobei das Regelsignal $u(t)$ nach Erreichen des Fixpunktes verschwindet (nichtinvasive Kontrolle wegen der AC-Kopplung der Eingangssignale).
\end{abstract}

zeigt ein Beispiel einer auf diese Weise herbeigeführten Fixpunktstabilisierung nach Einschalten der Regelung zur Zeit $t=8 \mathrm{~ms}$. Dabei fällt auf, dass in diesem Beispiel die Intensität des grünen Laserlichtes nach Ablauf der Transienten im stabilisierten Fall höher ist als im Chaosbereich. Diese Beobachtung konnte auch am Experiment bestätigt werden. Die Abhängigkeit der Fixpunktstabilisierung von den Filtergüten $Q_{1}=0.2 ; Q_{2}=0.5$; $Q_{3}=0.8 ; Q_{4}=1.1 ; Q_{5}=1.5$ ist in Abb 5.13a gezeigt, wobei die Stabilitätsgebiete entsprechend der vorher angegebenen Reihenfolge von dunkel nach hell gekennzeichnet sind. Wiederum ergeben sich die Stabilitätsdiagramme durch nummerische Integration der Zustandsgleichungen $(5.5 \mathrm{a}, \mathrm{b})$ für eine fest eingestellte Pumprate von $\mathrm{w}_{0}=1.243$. Die zugehörigen Verstärkungen seien auf die Werte $k_{1}=0.9$ und $k_{2}=1.7$ fixiert. Anhand dieser Abbildung erkennt man, dass die Filterqualität nicht zu hoch gewählt werden sollte, da dadurch die Stabilitätsgebiete wieder schrumpfen. In der Praxis verbietet sich eine zu hohe Güte, da die Filter dann sehr genau eingestellt werden müssen und $|T(f)| \approx 0$ für weite Einstellbereiche der Resonanzfrequenz teilweise schwierig zu bewerk- 

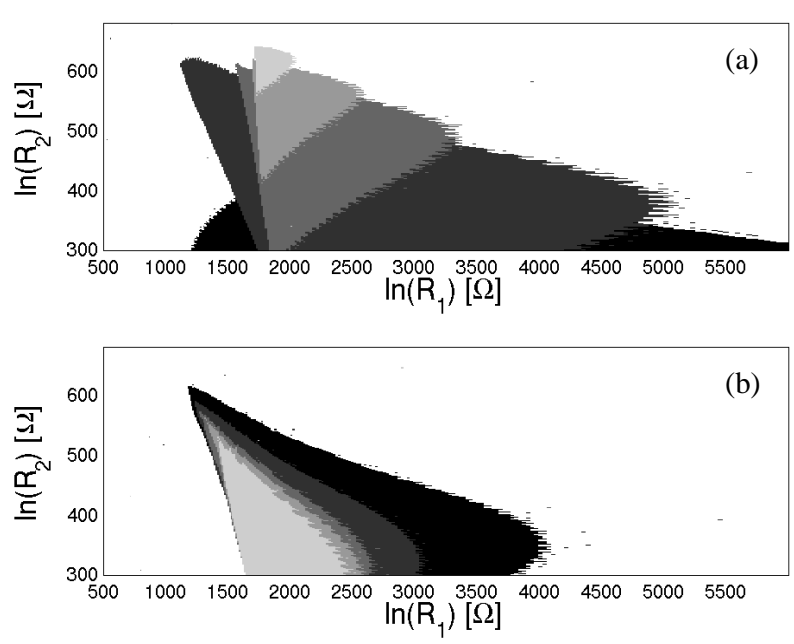

Abb. 5.13: Stabilitätsbereiche in der $R_{1}-R_{2}$-Parameterebene für die Resonanzfrequenzen $f_{1}, f_{2}$ der Kerbfilter (3.2) zur Regelung des Lasermodells. Parameterkombinationen, die keine Fixpunktstabilisierung erzielen, sind weiß dargestellt. (a) Die Stabilitätsbereiche schrumpfen mit wachsender Güte $Q_{j}$ (von dunkel nach hell) bei fest eingestellter Verstärkung. (b) Stabilitätsbereiche schrumpfen ebenfalls bei fester Güte $Q_{j}$ und gleichzeitiger Verstärkungserhöhung (von dunkel nach hell).

stelligen ist. Abb. 5.13b verdeutlicht zudem unter Verwendung von $k_{1}=1.1, k_{2}=1.6$; $k_{1}=1.3, k_{2}=1.4 ; k_{1}=1.5, k_{2}=1.3 ; k_{1}=1.7, k_{2}=1.3 ; k_{1}=1.9, k_{2}=1.3$ für die von dunkel nach hell markierten Gebiete, dass die Stabilitätsbereiche bei fest eingestellter Filtergüte $Q_{1}=Q_{2}=0.5$ am meisten vergrößert werden, wenn die Verstärkungsfaktoren $k_{1}$ und $k_{2}$ der beiden Kerbfilter unterschiedlich (asymmetrisch) und nicht zu groß gewählt sind. Das bestätigt noch einmal die Ergebnisse, die auch schon bei MDFC zu Tage traten.

\subsection{Phasensynchronisation}

Wird der Gedanke einer Regelung zu Gunsten einer Steuerung aufgegeben, so war bei den eigenen Experimenten keine Fixpunktstabilisierung mehr möglich. Stattdessen lag das Augenmerk auf der (chaotischen) Phasensynchronisation [129,130], wofür es lediglich einer festen Bedingung $\left|n \Phi_{d}-m \Phi_{r}\right|<$ const, $n, m \in \mathbb{N}$ zwischen der Phase $\Phi_{d}$ des treibenden und der Phase $\Phi_{r}$ des getriebenen Systems $[129,130]$ bedarf, die hierbei geeignet zu definieren sind. Die Amplituden der beiden Systeme sind hingegen unterschiedlich, wobei für chaotische Phasensynchronisation die Amplitude mindestens eines Systems chaotisch bleibt. In der Literatur lassen sich mehrere Publikationen finden, in denen Phasensynchronisation für verschiedene theoretische und experimentelle (chaotische) Systeme festgestellt wurde [43, 93, 130, 147]. Häufig standen in diesem Zusammenhang Laser im Fokus der experimentellen Untersuchungen. So wurde ein $\mathrm{CO}_{2}$-Laser analysiert, bei dem ein Teil der Ausgangsintensität dazu diente, einen innerhalb des Laserresonators platzierten elektrooptischen Modulator zu beeinflussen. Durch die damit bewirkte Veränderung der Resonatorverluste konnte bei geeigneter Parameterwahl Phasensynchronisation nachgewiesen werden [11,29]. Das gleiche Vorgehen führte auch für einen Nd:YAG-Laser zum Erfolg [94]. Zudem wurde Phasensynchronisation bei Halbleiterlasern [46] und in 
Laser-Anordnungen [40] festgestellt, z. B. bei zwei bidirektional gekoppelten Nd:YAGLasern [76]. Einschränkend muss allerdings gesagt werden, dass bei all den genannten Untersuchungen immer entweder zwei gleiche, bidirektional gekoppelte Laser oder aber Laser mit der beschriebenen Selbstmodulation verwendet wurden. Unter diesem Gesichtspunkt ist der experimentelle Nachweis chaotischer Phasensynchronisation für zwei unterschiedliche und lediglich unidirektional gekoppelte Systeme bislang ausgeblieben. Motiviert durch dieses fehlende experimentelle Beispiel habe ich den zur Verfügung stehenden kompakten frequenzverdoppelten Laser in dieser Hinsicht untersucht und im Zuge dieser Arbeit experimentell chaotische Phasensynchronisation an dem vorhandenen Lasersystem feststellen können. Zu diesem Zweck wurde der Pumpstrom des frequenzverdoppelten Lasers unter Verwendung des Aufbaus aus Abb. 5.15a durch ein sinusförmiges Generatorsignal moduliert. (Chaotische) Phasensynchronisation liegt in diesem Zusammenhang vor, wenn die Phasen der Schwingungen des Generators und die Intensitätsschwankungen des Lasers eine feste Beziehung zueinander aufweisen, während die Amplituden jeweils für sich ihrer eigenen chaotischen oder periodischen Dynamik folgen. Um die gemessenen Zeitreihen im Hinblick auf Phasensynchronisation zu untersuchen, muss allerdings zunächst eine Phase definiert werden, was bei einem chaotischen (Laser-)Signal nicht ohne weiteres möglich ist. Einen Spezialfall bilden hierbei Systeme, die nach einer zweidimensionalen Einbettung im wesentlichen einen Attraktor offenbaren, der in dieser niedrigdimensionalen Darstellung einer Spirale um einen festen Punkt im Zustandsraum ähnelt. Für die angesprochene zweidimensionale Einbettung eignet sich die Verwendung der Hilbert-Transformation bzw. des analytischen Signals ${ }^{6}$, dessen Real- und Imaginärteil gegeneinander aufgetragen werden. Auf diese Weise kann im Einzelfall rein visuell entschieden werden, ob Phasensynchronisation vorliegt oder nicht - je nachdem, ob in einer stroboskopischen Aufnahme die Dynamik des Lasers auf ein räumlich stark begrenztes Gebiet beschränkt bleibt. Für die Realisierung dieser stroboskopischen „Beleuchtung“ bietet es sich an, die Intensitätsschwankungen des Lasers z. B. bei den Nulldurchgängen der ansteigenden Flanke des Generators zu detektieren und entsprechend zu kennzeichnen. Ein Beispiel für dieses Vorgehen zeigt Abb. 5.14c, in der das phasensynchronisierte Lasersignal aus Abb. 5.14a in zwei Dimensionen eingebettet und die bei den Nulldurchgängen des Generatorsignals vorliegende Laserdynamik rot dargestellt ist. Allerdings muss eine so vorgenommene Klassifizierung weiter geprüft werden, da aufgrund der niedrigdimensionalen Einbettung falsche nächste Nachbarn er-

\footnotetext{
${ }^{6}$ Bei der Hilbert-Transformation der Funktion $f(x)$ handelt es sich um die lineare und zeitinvariante Integraltransformation

$$
H(y)=\frac{1}{\pi} \int_{-\infty}^{\infty} \frac{f(x)}{y-x} \mathrm{~d} x,
$$

bei der allerdings der Hauptwert des angegebenen Integrals ausgewertet werden muss. Im Detail wird hierdurch die Faltung der reellen Funktion $f(x)$ mit der Übertragungsfunktion $h(y)=(\pi y)^{-1}$ beschrieben. Hauptanwendungsgebiet ist die Extrahierung der Phase aus einem reellen Signal bzw. einer reellen Zeitreihe. Zudem kann gezeigt werden, dass mit Hilfe der Hilbert-Transformation $H(s)$, dem Signal $s$ und dem daraus resultierenden analytischen Signal $F(s)=s+i H(s)$ [116] eine optimale Einbettung in zwei Dimensionen vorgenommen werden kann.
} 


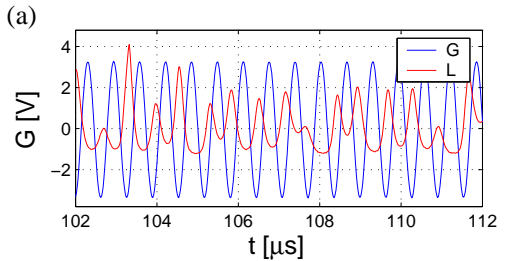

(d)

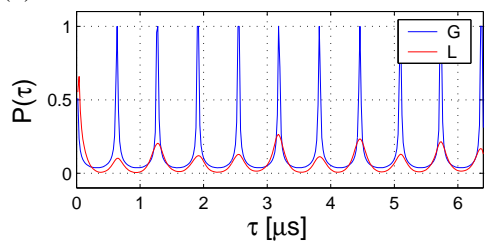

(b)

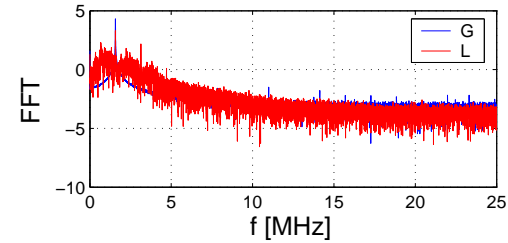

(e)

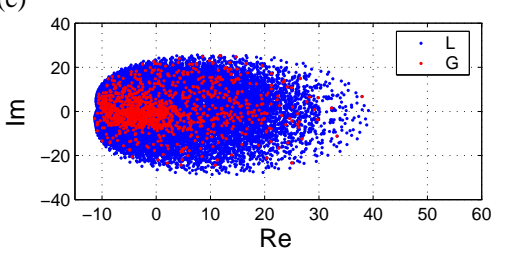

(c)

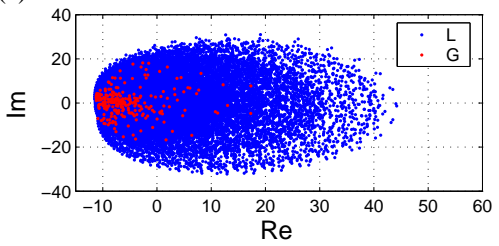

(f)

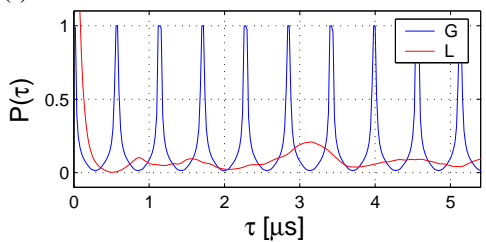

Abb. 5.14: (a) Experimentelles Beispiel für chaotische Phasensynchronisation bei fester Parameterwahl für den Generator und den Laser. (b) Zugehöriges Fourierspektrum, das die Übereinstimmung der Hauptfrequenzen des Laser- und Generatorsignals zeigt. (c) Zweidimensionale Einbettung des getriebenen Lasersignals aus (a) mit Hilfe des analytischen Signals. Die Nulldurchgänge der ansteigenden Flanke des Generatorsignals dienen der stroboskopischen „Beleuchtung“ der Laserdynamik (rot markiert). (d) Rekurrenzplot $P(\tau)$ vs. $\tau$ für das Laser- und das Generatorsignal aus (a). (e),(f) Beispiel für keine Phasensynchronisation, erkennbar an der (e) zweidimensionalen Einbettung sowie an den Rekurrenzverläufen. Die Bezeichnungen in (e),(f) stimmen mit denen aus (c),(d) überein.

zeugt werden könnten, die im Endeffekt zu einer verzerrten Darstellung der wirklichen Dynamik führen. Von daher ist eine mehrfache Einbettung in unterschiedlichen Dimensionen zu bevorzugen, um eine Entscheidung darüber zu treffen, ob wirklich (chaotische) Phasensynchronisation vorliegt oder nicht. Eine weitere Analyse kann neben der Prüfung der Übereinstimmung der Hauptfrequenzen in den Spektren des Treiber- und AntwortSystems (Abb. 5.14b) durch Rekurrenzplots ${ }^{7}$ vorgenommen werden [127]. Dieser Test beruht auf der Selbstähnlichkeit der (chaotischen) Signale, für die die Rekurrenz

$$
P(\tau)=\frac{1}{N-\tau} \sum_{i=1}^{N-\tau} \Theta\left(\epsilon-\left\|\mathbf{x}_{j}-\mathbf{x}_{j+\tau}\right\|\right)
$$

nach Verschieben um die Zeit $\tau$ ein lokales Maximum besitzt. Die Heaviside-Funktion $\Theta\left(\epsilon-\left\|\mathbf{x}_{j}-\mathbf{x}_{j+\tau}\right\|\right)$ liefert in diesem Zusammenhang den Funktionswert 1, wenn die Punkte $\mathbf{x}_{j}$ und $\mathbf{x}_{j+\tau}$ des (rekonstruierten) Zustandsraumes weniger als $\varepsilon$ voneinander entfernt

\footnotetext{
${ }^{7}$ In einem Rekurrenzplot werden die Zeiten grafisch dargestellt, zu denen eine betrachtete Trajektorie im Phasenraum wieder in die Nachbarschaft des Ausgangspunktes zur Zeit $t_{0}$ zurückkehrt. Möglich ist diese Rekurrenz von Zuständen, da viele Prozesse in der Natur periodischen bzw. oszillatorischen Charakters sind und sich selbst für nichtlineare und chaotische Systeme Zustände nach einer bestimmten Zeitspanne aufgrund des Determinismus und der Ergodizität wieder unendlich nahe kommen.
} 
sind. Andernfalls nimmt die Heaviside-Funktion den Wert 0 an. Da das treibende Generatorsignal periodisch ist, liegt nach dem Verschieben um eine Periode $T=\tau$ wieder dasselbe Signal vor, woraufhin $P(\tau)=1$ ist. Bei einem chaotischen Signal wird $P(\tau)=1$ nicht erreicht, sondern bleibt auf Bereiche $P(\tau)<1$ beschränkt, wobei benachbarte Maxima jeweils verschiedene Funktionswerte besitzen. Zeigt nun Gl. (5.26) für das Treiberund das Antwortsignal zu den gleichen Rekurrenzzeiten $\tau$ Maxima, so liegt Phasensynchronisation vor. Ein Beispiel für diesen Sachverhalt ist in der Abb. 5.14d dargestellt, die die Rekurrenzverläufe $P(\tau)$ für das Laser- und das Generatorsignal in Abhängigkeit von der Rekurrenzzeit $\tau$ zeigt. Dass die Phasensynchronisation chaotisch ist, kann anhand der unterschiedlichen Höhen der Rekurrenzmaxima des Lasersignals erkannt werden. Im Vergleich hierzu zeigen die Rekurrenzverläufe in Abb. 5.14f zu nicht übereinstimmenden Rekurrenzzeiten $\tau$ Maxima. Es handelt sich demzufolge um ein Beispiel keiner vorhandenen Phasensynchronisation. Dieser Sachverhalt kann auch der stroboskopischen Abb. 5.14e 8 entnommen werden. Dabei bleibt die stroboskopisch durch den Generator „,beleuchtete" Laserdynamik nicht auf ein enges räumliches Gebiet beschränkt, d. h. die Phasen des Generators und des Lasers sind nicht miteinander gekoppelt.

Für die Ermittlung der Rekurrenzverläufe wurden sowohl das Generator- als auch das Lasersignal entsprechend der Dimensionsanalyse aus Abschnitt 5.4 in fünf Dimensionen eingebettet. Die anschließende Berechnung von $P(\tau)$ gemäß Gl. (5.26) hängt allerdings kritisch von der $\epsilon$-Umgebung ab. Wird $\epsilon$ zu klein gewählt, sind die Amplituden des resultierenden $P(\tau)$ für das (chaotische) Lasersignal sehr klein und in Gegenwart von (starkem) Messrauschen u. U. schwer zu detektieren. Wird andererseits $\epsilon$ zu groß gewählt, liegen nach einer Einbettung bei schnell abgetasteten Signalen entsprechend viele Punkte innerhalb der gewählten $\epsilon$-Umgebung. Demzufolge zeigt $P(\tau)$ für einen ausgedehnten Bereich von $\tau$-Werten einen konstanten, maximalen Verlauf mit $P(\tau)=1$. Im eigenen Experiment wurden alle $20 \mathrm{~ns}$ Generator- und Lasermesswerte akquiriert. Für die Auswertung hat sich $\epsilon=0.05 \Delta$ als sinnvoll herausgestellt, wobei $\Delta$ der Durchmesser des eingebetteten Attraktors in der Raumrichtung mit der größten Ausdehnung ist.

Neben diesen Beispielen für einen festen Parametersatz (Laser: Pumpstrom sowie die Temperatur des zweiten Spiegels und der Pumpdiode, Generator: Frequenz und Amplitude des verwendeten Sinussignals) ist natürlich eine ausgedehnte Messung im Parameterraum zur genauen Lokalisierung des Auftretens der chaotischen Phasensynchronisation von Interesse. Unglücklicherweise behindert die Instationarität des Lasersystems eine solche ausgedehnte Messung und lässt nur eine sehr eingeschränkte Äquivalenzmessung mit geringerer Auflösung zu. Diese liegt in Form eines $40 \times 40$-Rasters vor, wobei die Amplitude und die Frequenz des Generators verändert und die resultierende Laserdynamik detektiert wurde. Für die Auswertung kommt die Kreuzkorrelation

$$
C=\frac{\left\langle\bar{P}_{L}(\tau) \bar{P}_{G}(\tau)\right\rangle}{\sigma_{L} \sigma_{G}}
$$

${ }^{8}$ Zweidimensionale Einbettung mit Hilfe der Hilbert-Transformation (5.25) bzw. des analytischen Signals. 
(a)

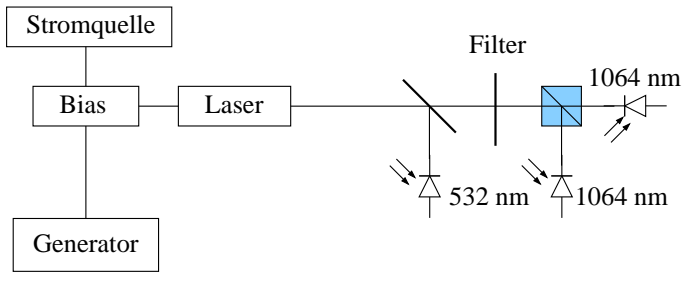

(b)

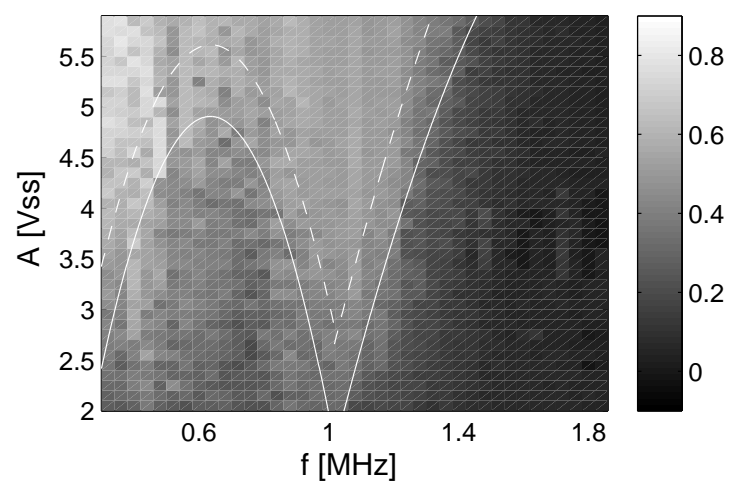

Abb. 5.15: (a) Experimenteller Aufbau für die Synchronisationsmessung am frequenzverdoppelten Laser. Als Messsignal für das getriebene Lasersystem stehen die infraroten oder die grüne Intensität zur Verfügung. (b) Dargestellt ist der Korrelationskoeffizient $C$ vs. Generatorfrequenz $f$ und Generatoramplitude $A$. Für die Berechnung des Korrelationskoeffizienten wurde das Generatorsignal und die grüne Intensität verwendet. $\mathrm{Zu}$ erkennen ist eine typische Arnold-Zunge als hell gefärbtes Synchronisationsgebiet. Chaotische Phasensynchronisation ist an den Rändern, periodische Phasensynchronisation in den inneren Bereichen der Arnold-Zunge zu finden.

zum Einsatz. Bei $\bar{P}_{L}(\tau), \bar{P}_{G}(\tau)$ handelt es sich um die von dem jeweiligen Mittelwert befreiten Rekurrenzverläufe $P_{L}(\tau), P_{G}(\tau)$ des Laser- bzw. Generatorsignals. $\sigma_{G}$ und $\sigma_{L}$ bezeichnen die zugehörigen Standardabweichungen von $\bar{P}_{L}(\tau)$ und $\bar{P}_{G}(\tau)$. Liegt Phasensynchronisation vor, so hat die Kreuzkorrelation $C$ (5.27) ein Maximum bzw. einen hohen Wert. Besitzen $\bar{P}_{G}(\tau)$ und $\bar{P}_{L}(\tau)$ nicht an derselben Stelle Maxima, so hat die resultierende Kreuzkorrelation einen geringen Wert. Im einzelnen ergibt sich mit diesem Vorgehen der in Abb. 5.15b präsentierte Zusammenhang, der den Korrelationskoeffizienten $C$ zwischen dem Laser- und dem Generatorsignal in der $f$-A-Parameterebene des Generators darstellt. Im wesentlichen ergibt sich Synchronisation in dem hellen, keilförmigen Gebiet (ArnoldZunge), an dessen Rand die chaotische Phasensynchronisation angesiedelt ist. Zur Mitte der Arnold-Zunge hin wird die chaotische Phasensynchronisation von periodischer Phasensynchronisation abgelöst. Die Unterscheidung zwischen chaotischer und periodischer Phasensynchronisation kann in diesem Zusammenhang entweder durch einen Vergleich der Höhen der Rekurrenzmaxima (im Falle eines periodischen Signals sind alle Maxima gleich hoch) entsprechend Abb. 5.14 oder mit Hilfe der Autokorrelationsfunktion vorgenommen werden. Dabei wird ein Signal als periodisch angenommen, wenn die zugehörige Autokorrelationsfunktion (mehrmals) den Wert 0.89 überschreitet. 


\section{Kapitel 6}

\section{Räumlich ausgedehnte Systeme}

\subsection{Allgemeines}

Bisher wurde sowohl MDFC als auch NFF lediglich auf (räumlich konzentrierte) zeitkontinuierliche Systeme angewendet. Der Übergang zu räumlich ausgedehnter Dynamik kann in diesem Zusammenhang am einfachsten durch die Betrachtung von Abbildungsgittern vorgenommen werden, wodurch gleichzeitig auch ein Wechsel von (zeit)kontinuierlicher zu (zeit)diskreter Dynamik stattfindet. Häufig wird unter diesem Gesichtspunkt als einfachster Repräsentant zeitdiskreter Systeme die logistische Abbildung

$$
f\left(x_{n}\right)=x_{n+1}=4 \lambda x_{n}\left(1-x_{n}\right)+u_{n}, \quad \lambda \in[0,1]
$$

gewählt, die wiederum durch Kopplung mehrerer Abbildungen auf räumliche Systeme verallgemeinerbar ist. Die Dynamik von Gl. (6.1) verändert sich dabei durch Variation des Parameters $\lambda$ und deaktivierter Regelung $u_{n}$ vom Fixpunkt, bzw. Periode-1-Orbit über verschiedene periodische Orbits bis hin zum Chaos $(\lambda>0.89)$, das für $\lambda=1$ am stärksten ausgeprägt ist und im folgenden mittels des Kontrollsignals $u_{n}$ zugunsten des Fixpunktes $x_{0}=1-(4 \lambda)^{-1}$ eliminiert werden soll. Dabei stellt sich wieder die Leistungsfähigkeit der MDFC-Rückkopplung heraus, die klare Vorteile gegenüber der herkömmlichen TDASKontrolle

$$
u_{n}=k\left(x_{n-\tau}-x_{n}\right)
$$

besitzt. So kann mit MDFC und zwei unterschiedlichen Verzögerungszeiten der Periode1-Orbit der logistischen Abbildung (6.1) (entspricht einem Fixpunkt) noch bis $\lambda=0.98$ stabilisiert werden, bei Verwendung von vier unterschiedlichen Verzögerungszeiten sogar für $\lambda=1$. Mit TDAS ist dieser Orbit nach meinen Untersuchungen nur bis zu einem Wert von $\lambda=0.91$ stabilisierbar. Aufschluss über diesen Sachverhalt vermittelt Abb. 6.1, in der TDAS-Kontrolle für $\lambda=0.98$ nicht mehr zu einer Stabilisierung des Fixpunktes führt. 
(a)

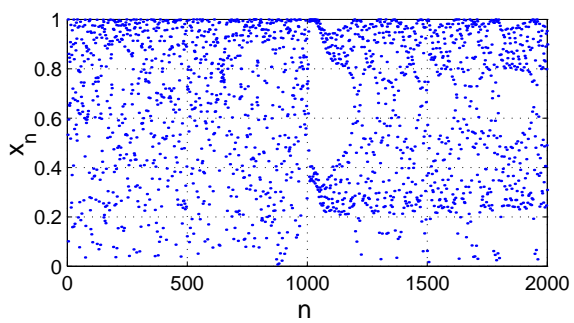

(b)

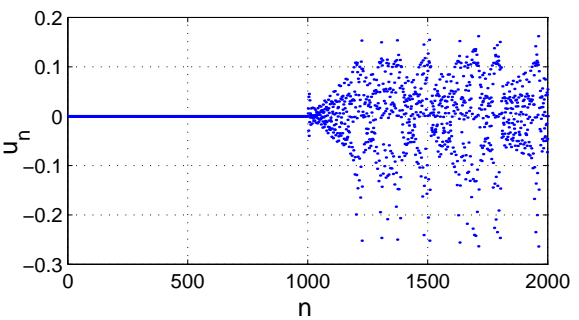

Abb. 6.1: (a) TDAS-Kontrolle der logistischen Abbildung aus Gl. (6.1) für $\lambda=$ 0.98. Die Aktivierung der Regelung mit $\tau=2$ erfolgt für $n=10000 . \tau \geq 2$ ist notwendig, damit es sich nicht um einen verstärkten D-Kontroller handelt $(\tau=1)$.

(b) Zugehöriges Regelsignal. Es ist keine Stabilisierung des Periode-1 Orbits möglich.

Der Übergang zu räumlich ausgedehnten Systemen kann nun z. B. über die Kopplung mehrerer logistischer Abbildungen zu einem Ring (d.h. periodische Randbedingungen) vorgenommen werden, was in entsprechender Verallgemeinerung von Gl. (6.1) zu

$$
x_{n+1}(i)=(1-\epsilon) f\left(x_{n}(i)\right)+\frac{\epsilon}{2}\left[f\left(x_{n}(i-1)\right)+f\left(x_{n}(i+1)\right)\right]+u_{n}(i)
$$

für den Ort $i$ mit $\epsilon \in[0,1]$ führt. Hierbei handelt es sich um eine bidirektionale Kopplung, bei der jede logistische Abbildung mit dem Kopplungsfaktor $\epsilon$ auf seine beiden nächsten Nachbarn wirkt. Diese Art des räumlich ausgedehnten Systems unterlag schon vielfältigen Untersuchungen bezüglich Bifurkationsverhalten [74,75], Synchronisation [61] und Regelung [79-81, 110]. Für die Stabilisierung des kompletten räumlichen Gebietes sei an dieser Stelle das Regelsignal

$$
u_{n}(i)=\sum_{j=1}^{4} k_{m a} x_{n-\tau_{m}}(i)-k_{m b} x_{n}(i)
$$

mit den Verstärkungen $k_{m a}, k_{m b}$ und der zeitverzögerten Messgröße $x_{n-\tau_{m}}(i)$ am Ort $i$ angewendet, das nur verschwindet, wenn jeder Oszillator seinen (entsprechend für alle identischen) Fixpunkt erreicht hat. Ein Beispiel für solch eine erfolgreiche Stabilisierung veranschaulicht Abb. 6.2, in der 50 logistische Abbildungen für $\lambda=1$, d. h. voll entwickeltes Chaos in Form einer Ringtopologie miteinander verknüpft sind. Nach Beginn der Regelung (6.4) für $n>10000$ wird das komplette dynamische Gebiet auf den systemeigenen Fixpunkt stabilisiert, das Regelsignal jedes Oszillators verschwindet (Abb. 6.2b). Die Wirksamkeit von mehreren Verzögerungszeiten wurde inzwischen für iterierte Systeme unabhängig von mir in [92] dokumentiert, wodurch MDFC bestätigt wird. Das Problem bei der Regelung iterierter Abbildungen mit Hilfe von MDFC ist allerdings, dass es sich bei den Verzögerungszeiten $\tau_{m}$ sehr schnell um ganzzahlige Vielfache voneinander handelt, wodurch der Unterschied zu (E)TDAS unter Umständen nur noch durch die individuellen Vorfaktoren gegeben ist. Von daher soll die Welt der iterierten Systeme nach diesem kurzen Intermezzo wieder zu Gunsten der zeitkontinuierlichen Dynamik verlassen werden, die 
(a)

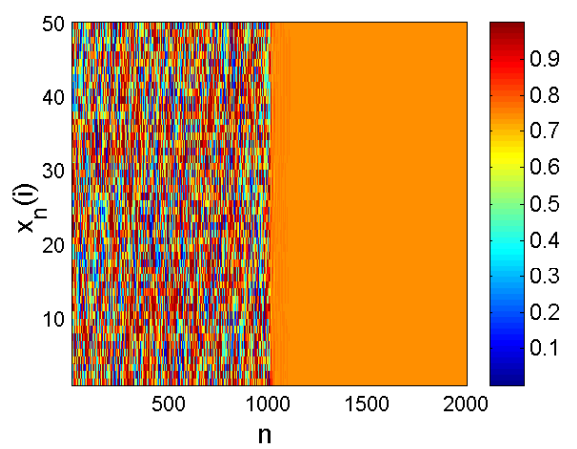

(b)
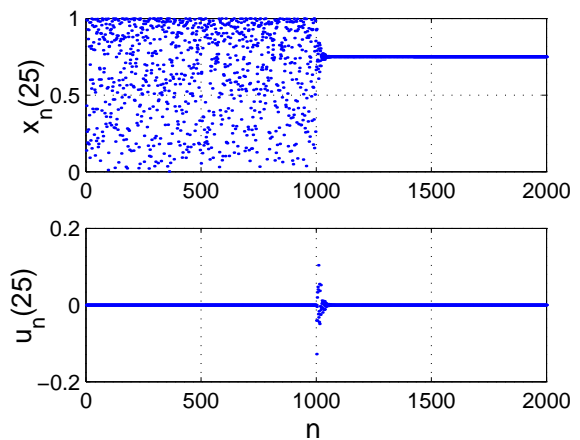

\begin{abstract}
Abb. 6.2: Stabilisierung eines räumlich ausgedehnten Systems, bestehend aus 50 $\mathrm{zu}$ einem Ring gekoppelten logistischen Abbildungen (6.3) mit $\lambda=1, \epsilon=0.5$. Die eingesetzte MDFC-Regelung aus Gl. (6.4) mit vier unterschiedlichen Verzögerungszeiten $\tau_{1}=2, \tau_{2}=4, \tau_{3}=5, \tau_{4}=7$ und den symmetrischen Verstärkungsfaktoren $k_{1 a}=k_{1 b}=0.3, k_{2 a}=k_{2 b}=0.2, k_{3 a}=k_{3 b}=0.1, k_{4 a}=k_{4 b}=0.2$ wird nach 1000 Iterationen aktiviert. Dabei wird jede logistische Abbildung nur auf sich selbst zurückgekoppelt, der Einfluss der nächsten Nachbarn erfolgt lediglich über den Kopplungsparameter $\epsilon$.
\end{abstract}

in räumlich ausgedehnten Systemen [109,111, 112] ebenfalls raum-zeitliches Chaos in Hinblick auf irreguläre Wellenfronten, Amplituden, turbulente Dynamik oder Spiralwellen [37] zeigt. Beispiele für räumliche, komplexe Dynamik sind Flüssigkeitskristalle [48, 95, 103], die Carbon-Monoxid-Oxidation auf einer (110)-Pt Oberfläche [21-23, 66, 77], das Herzgewebe [45], elektrochemische Gleichgewichtsreaktionen [152] oder zweidimensionale Anordnungen von elektronischen Oszillatoren [60].

In vielen Situationen ist raum-zeitliches Chaos allerdings unerwünscht. Man denke in diesem Zusammenhang z. B. an das menschliche Herz, das im gesunden Zustand laufende Wellen generiert, die sich um den Herzmuskel herum ausbreiten. Als Folge von Unregelmäßigkeiten im Herzgewebe oder Krankheiten spalten sich diese Wellen in mehrere Spiralwellen auf, die den Beginn von Herzrhythmusstörungen und Fibrilation kennzeichnen bzw. nach sich ziehen. Aber auch in der Technik treten störende Spiralwellen bei vielfältigen Anwendungen auf, beispielsweise bei der Elektrokatalyse in Treibstoffzellen, Korrosion, der elektrochemischen Bearbeitung von Metallen oder aber bei der Generation von Mustern und Clustern, die häufig mit komplexer raum-zeitlicher Dynamik einhergehen. All diese Beispiele haben gemein, dass (Regelungs-)Methoden benötigt werden, um die Dynamik im Hinblick auf die gewünschte Zieldynamik zu beeinflussen. Aus diesem Grund wurden in den vergangenen 10 Jahren verschiedene Strategien verfolgt, um raum-zeitliches Chaos zu beherrschen und die entsprechenden Systeme handhabbar zu machen [96]. Da es um räumlich ausgedehnte Systeme geht, können Randbedingungen oder kleine räumliche Inhomogenitäten dazu verwendet werden, die Systemdynamik in bestimmten Bereichen zu kontrollieren. Solch statische Methoden kamen z. B. in [105] 
zum Einsatz, um Spiralwellen zu zerstören oder um stationäre bzw. atmende Muster zu erzeugen [56]. Darüber hinaus können durch diese Technik Spiralwellen zum Driften veranlasst [25] bzw. unterdrückt [13] sowie bestimmte Wellenmuster erzeugt werden [42].

Diesen statischen Methoden stehen aktive Möglichkeiten der Manipulation gegenüber, die z. B. via externer Modulation [12, 83, 89, 114, 133, 159, 161] oder in Form von elektrischen Pulsen bei der Zerstörung von Spiralwellen eingesetzt werden können, z. B. im Herzgewebe (Defibrilation) [107]. Nachteile der Defibrilation sind allerdings die nicht zu vermeidenden Folgeschäden wie Rippenbrüche etc., die von zu stark gewählten Stromstößen herrühren. Unter diesem Gesichtspunkt wären angepasste Regelungsmethoden diesen Steuerungen aufgrund der geringeren benötigten Kontrollkraft vorzuziehen. Generell wurden zur Regelung raum-zeitlicher Dynamik im wesentlichen die bereits genannten Methoden OGY und (E)TDAS [20, 28, 55, 109, 111] sowie einfache Proportionalkontrolle verwendet. So ist es unter anderem mit Proportionalregelung unter Benutzung einer oder mehrerer Observablen des Systems für die Erzeugung des Regelsignals möglich, auf Spiralwellen Einfluss zu nehmen. Allerdings ist dieses Ergebnis nicht für alle Systeme verallgemeinerbar.

Das Kontrollsignal kann in diesem Zusammenhang sowohl lokal als auch global zum Einsatz kommen [42,111]. Lokale Kontrolle berücksichtigt in ausgewählten kleinen Gebieten ermittelte Signale, die auch nur lokal zur Regelung herangezogen werden. Globale Kontrolle umfasst das gesamte dynamische Gebiet, in dem entweder ortsspezifisch individuelle oder ein gemitteltes Signal für den gesamten Bereich angewendet wird. Es hat sich allerdings schon mehrfach gezeigt, dass die Ergebnisse, die sowohl lokale als auch globale Rückkopplungen herbeiführen, in weiten Bereichen sehr ähnlich sind [20]. Ein weiterer Regelungsmechanismus wurde in $[51,53]$ einer eingehenden Untersuchung unterzogen, bei der lokal ein Stimulus in der Belousov-Zhabotinsky-Reaktion angewendet wurde, sobald sich Wellenfronten durch einen bestimmten Messpunkt bewegt haben. Chaos-Kontrolle unter Verwendung zeitverzögerter Dynamik wurde, wie bereits mehrfach in diesem Kontext genannt, zuerst von Pyragas vorgeschlagen [117], wobei sich herausstellte, dass damit eine effiziente Regelungsmethode unter dem Gesichtspunkt der praktischen Anwendung gegeben ist. Das Regelsignal wird entsprechend der Formel (2.10) generiert. Ähnlich zu vorher hat sich auch für räumlich ausgedehnte Systeme herausgestellt, dass die Berücksichtigung von ganzzahligen Vielfachen der einen festen Verzögerungszeit $\tau$ (ETDAS (2.11)) zu stabileren Lösungen führt [20]. Anwendungen von TDAS im Hinblick auf Spiralwellen-Probleme und Kontrolle von raum-zeitlichem Chaos beinhalten Anordnungen von Halbleiterlasern [97], Spiralwellen in sphärischen Oberflächen [162], Instabilitäten im Herzgewebe [125], raumzeitliches Spiking [47], Spiralpfade in der Belousov-Zhabotinsky Reaktion [149], oszillierende Reaktions-Diffusions Systeme [24] und Systeme mit anregbarem Rand in verschiedenen Formen [158,160]. Darüber hinaus wurde die Effizienz der zeitverzögerten Regelungen durch räumliche Filterung des Regelsignals wesentlich verbessert [15,27]. Generell wurde allerdings in [47] festgestellt, dass sowohl die OGY-Methode als auch (E)TDAS nur für instabile Orbits mit wenigen instabilen Richtungen den gewünschten Erfolg erzielt. 


\subsection{Die Ginzburg-Landau-Gleichung}

In den kommenden Abschnitten werde ich demonstrieren, dass MDFC auch dazu verwendet werden kann, raum-zeitliches Chaos sowohl global als auch lokal zu manipulieren und zu stabilisieren. Zur Illustration dieser Vorgänge dient die zweidimensionale komplexe Ginzburg-Landau-Gleichung (GLE)

$$
\partial_{t} f=(1+i a) \nabla^{2} f+f-(1+i b) f|f|^{2}+g(\mathbf{x}) u
$$

mit einem externen Regelsignal $u=u(t)$ zur Kontrolle und Manipulation des auftretenden Chaos. $\partial_{t}$ und $\nabla$ bezeichnen in diesem Zusammenhang die zeitliche und die räumliche Ableitung und $g(\mathbf{x})$ ist eine räumliche Funktion, die das Kontrollsignal $u$ zusätzlich (räumlich) moduliert. Bei der GLE (6.5) handelt es sich um eine universelle Modellgleichung für alle Systeme, die eine superkritische Hopf-Bifurkation durchlaufen haben. Nummerisch integriert wird Gl. (6.5) in der Zeit mit Hilfe eines Runge-Kutta-Lösers 4. Ordnung und spektral im Raum $(90 \times 90$ Zellen, $\Delta x=\Delta y=1)$. Abhängig von den Parameterwerten $a$ und $b$ zeigt die komplexe Lösung $f=f(\mathbf{x}, t)$ der Gl. (6.5) dann verschiedene Arten raumzeitlichen Chaos, u. a. aber auch einen instabilen Fixpunkt $f(\mathbf{x}, t)=0$, der im Gegensatz zu (E)TDAS für alle Parameter $a, b$ durch einen P-Regler oder durch MDFC stabilisiert werden kann (siehe Anhang ab Seite 125). Weiterhin umfassen laufende ebene Wellen

$$
f(\mathbf{x}, t)=f_{0} e^{i\left(\mathbf{k}_{0} \cdot \mathbf{x}-\omega_{0} t\right)}
$$

mit Wellenvektor $\mathbf{k}_{\mathbf{0}}$, Frequenz $\omega_{0}$ und Amplitude $f_{0}$ das Spektrum der möglichen (instabilen) Lösungen der GLE (Stabilitätsanalyse im Anhang ab Seite 125). Einsetzen von Gl. (6.6) in Gl. (6.5) führt zu den Relationen $\omega_{0}=k_{0}^{2}(a-b)+b$ und $f_{0}=\sqrt{1-k_{0}^{2}}$, wobei $k_{0}^{2}=\mathbf{k}_{\mathbf{0}} \cdot \mathbf{k}_{\mathbf{0}} \leq 1$ ist. Im eindimensionalen Fall können diese im chaotischen Attraktor eingebetteten instabilen Orbits (UPOs) durch TDAS stabilisiert werden [19,28]. Für höherdimensionale Fälle ist wiederum die für das Funktionieren von (E)TDAS bereits in Abschnitt 4.4 angesprochene Torsion [71,99] notwendig, die allerdings für $a b<-1$ nicht garantiert ist, so dass diese Regelungen dann nicht mehr erfolgreich sind [55]. Im weiteren Verlauf werde ich zeigen, dass diese Beschränkung mit MDFC und asymmetrischen Vorfaktoren durchbrochen und Stabilitätsgebiete durch die Verwendung mehrerer Verzögerungszeiten ausgedehnt werden können.

\subsection{Stabilisierung ebener Wellen}

Zunächst sei der Fall der homogenen Rückkopplung mit $g(\mathbf{x})=1$ betrachtet. Um instabile ebene Wellen zu stabilisieren, kommt das globale Regelsignal

$$
u(\mathbf{x}, t)=\sum_{m=1}^{M} k_{m a} f\left(\mathbf{x}, t-\tau_{m}\right)-k_{m b} f(\mathbf{x}, t)
$$


zum Einsatz. Unter der Annahme, dass sich die kontrollierten ebenen Wellen durch

$$
f(\mathbf{x}, t)=f_{c} e^{i\left(\mathbf{k}_{\mathbf{c}} \cdot \mathbf{x}-\omega_{0} t\right)}
$$

beschreiben lassen, liefert ein Einsetzen dieses Ausdrucks in Gl. (6.7) das Ergebnis

$$
u(\mathbf{x}, t)=\left(\sum_{m=1}^{M} k_{m a} e^{-i \omega_{0} \tau_{m}}-k_{m b}\right) f(\mathbf{x}, t)=T\left(\omega_{0}\right) f(\mathbf{x}, t)
$$

mit der Übertragungsfunktion $T\left(\omega_{0}\right)$. Zusammen mit Gl. (6.8) führt dann wiederum Gl. (6.9) für die GLE (6.5) zu

$$
\begin{aligned}
1-k_{c}^{2}-f_{c}^{2}+\operatorname{Re}\left(T\left(\omega_{0}\right)\right) & =0 \\
\omega_{0}-a k_{c}^{2}-b f_{c}^{2}+\operatorname{Im}\left(T\left(\omega_{0}\right)\right) & =0,
\end{aligned}
$$

wobei $k_{c}^{2}=\mathbf{k}_{\mathbf{c}} \cdot \mathbf{k}_{\mathbf{c}}$ ist. $\operatorname{Im}\left(T\left(\omega_{0}\right)\right)$ und $\operatorname{Re}\left(T\left(\omega_{0}\right)\right)$ bezeichnen an dieser Stelle den Imaginärund den Realteil der Übertragungsfunktion $T\left(\omega_{0}\right)$ für die Frequenz $\omega_{0}$. Werden beide Bedingungen aus Gl. (6.10) miteinander kombiniert, so kann die Amplitude $f_{c}$ eliminiert werden, wodurch sich

$$
k_{c}^{2}=k_{0}^{2}+\Delta k^{2}
$$

mit

$$
\Delta k^{2}=\frac{b \operatorname{Re}\left(T\left(\omega_{0}\right)\right)-\operatorname{Im}\left(T\left(\omega_{0}\right)\right)}{b-a}
$$

ergibt. $\Delta k^{2}$ beschreibt in diesem Zusammenhang die aufgrund der aktiven Regelung auftretende Wellenzahlverstimmung. Da $k_{0}^{2} \leq 1$ ist, folgt $k_{c}^{2} \geq 0$ solange die Wellenzahlverstimmung auf Bereiche $\Delta k^{2} \geq-1$ beschränkt bleibt. Wird die Bedingung

$$
b \operatorname{Re}\left(T\left(\omega_{0}\right)\right)=\operatorname{Im}\left(T\left(\omega_{0}\right)\right)
$$

erfüllt, verschwindet $\Delta k^{2}$ und es wird eine ebene Welle mit der Wellenzahl $k_{c}=k_{0}$ stabilisiert. Da die Frequenz $\omega_{0}$ und die Wellenzahl mit denen der (instabilen) Welle des freilaufenden Systems übereinstimmen, liegt der einzige Unterschied in der Amplitude, die sich in Gegenwart einer aktivierten Regelung zu $f_{c}=\sqrt{f_{0}^{2}+\operatorname{Re}\left(T\left(\omega_{0}\right)\right)}$ berechnen lässt.

Die Stärke der Wellenzahlverstimmung hängt von der Gestalt der Übertragungsfunktion ab, die bei MDFC mit Hilfe der Parameter $k_{m a}, k_{m b}, k_{0}$ und $\tau_{m}$ flexibel angepasst werden kann. Um diese Abhängigkeit zu illustrieren, ist in Abb. 6.3a und 6.3b der Wert der Wellenzahlverstimmung $\Delta k^{2}$ graukodiert gegenüber den Kontrollparametern $\tau_{1}$ und $k_{1 b}$ für MDFC mit einer und zwei Verzögerungszeiten dargestellt. Unterhalb eines kritischen Verstärkungswertes $k_{1 b}$ versagt die Kontrolle und die betrachtete ebene Welle bleibt instabil (hell-graue Bereiche mit kleiner Verstärkung in Abb. 6.3). Kontrolle mit $\Delta k=0$ tritt für die $\bigcap$-förmigen Parameterkombinationen von $\tau_{1}$ und $k_{1 b}$ auf. Da die Parameterwerte 

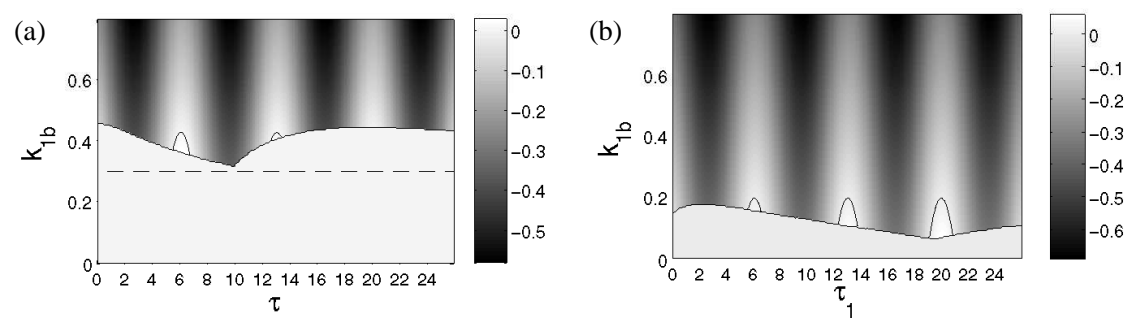

\begin{abstract}
Abb. 6.3: Stabilisierung von ebenen Wellen (6.6) für die GLE (6.5) mit den Parametern $a=1.1$ und $b=-1$ mit Hilfe des Regelsignals (6.7). Innerhalb der strukturierten grauen Gebiete der $\tau_{1}-k_{1 b}$-Parameterebene ist die Stabilisierung erfolgreich, wobei die Wellenzahlverstimmung $\Delta k^{2}$ (6.12) grau skaliert aufgetragen ist und für die $\bigcap$-förmigen Parameterkombinationen verschwindet. (a) Regelung mit einer Verzögerungszeit und $k_{1 a}=0.3$. Die gestrichelte Linie markiert den Fall der Rückkopplung mit symmetrischen Vorfaktoren (TDAS, $k_{1 a}=k_{1 b}$ ), die nicht in der Lage ist, die ebene Welle zu stabilisieren. (b) MDFC mit zwei Verzögerungszeiten und fest gewählten Kontrollparametern $k_{1 a}=0.3, k_{2 a}=0.1, k_{2 b}=0.3, \tau_{2}=7.2$.
\end{abstract}

der GLE in diesem Fall zu $a=1.1$ und $b=-1$ gewählt sind, ist das in [55] hergeleitete Kontrollierbarkeitskriterium $a b>-1$ für TDAS nicht erfüllt. Folglich scheitert die Kontrolle mit symmetrischen Vorfaktoren $k_{1 a}=k_{1 b}$, was anhand der gestrichelten Linie $k_{1 b}=0.3=k_{1 a}$ in Abb. 6.3 dokumentiert ist. Im Gegensatz dazu ermöglicht eine asymmetrische Wahl der Vorfaktoren $k_{1 a} \neq k_{1 b}$ bei entsprechend großer Verstärkung $k_{1 b}$ die Stabilisierung einer ebenen Welle mit der gleichen Wellenzahl $k_{0}$ (d.h. $\Delta k=0$ ) wie die der freilaufenden (instabilen) Lösung der GLE.

Analog zu den vorher vorgestellten Ergebnissen vergrößern sich die Stabilitätsgebiete bei der Anwendung weiterer, unterschiedlicher Verzögerungszeiten. Ein Beispiel für $\tau_{2}=7.2$ ist in Abb. $6.3 \mathrm{zu}$ sehen. Die Phasengeschwindigkeit

$$
v_{c}=\frac{k_{c}}{\omega_{0}}=\frac{1}{\omega_{0}} \sqrt{k_{0}^{2}+\Delta k^{2}}
$$

dieser ebenen Welle lässt sich durch die Regelung je nach gewählter Invasivität verändern. Sind die Regelparameter zu $\Delta k=0$ gewählt, ergibt sich $v_{c}=v_{0}=k_{0} / \omega_{0}$.

\title{
6.4 Inhomogene Rückkopplung
}

Wird das Kontrollsignal $u$ aus Gl. (6.7) nicht homogen angewendet, sondern durch die Funktion $g(\mathbf{x}) \neq 0$ räumlich moduliert, muss der Ansatz der harmonischen Welle (6.8) zugunsten der Annahme

$$
f(\mathbf{x}, t)=f_{c} e^{i\left(\mathbf{k}_{\mathbf{c}} \cdot \mathbf{x}+h(\mathbf{x})-\omega_{0} t\right)}
$$


modifiziert werden. Die Phasenfunktion $h(\mathbf{x})$ berücksichtigt in diesem Zusammenhang das Auftreten von anharmonischen Termen, die aus der Aktivierung der Regelung resultieren. Einsetzen von Gl. (6.15) in die GLE (6.5) führt mit $u(\mathbf{x}, t)=g(\mathbf{x}) T\left(\omega_{0}\right) f(\mathbf{x}, t)$ auf

$$
\begin{aligned}
1-\left(k_{c}+h^{\prime}\right)^{2}-f_{c}^{2}-a h^{\prime \prime}+g(\mathbf{x}) \operatorname{Re}\left(T\left(\omega_{0}\right)\right) & =0 \\
\omega_{0}-a\left(k_{c}+h^{\prime}\right)^{2}-b f_{c}^{2}+h^{\prime \prime}+g(\mathbf{x}) \operatorname{Im}\left(T\left(\omega_{0}\right)\right) & =0,
\end{aligned}
$$

mit $k_{c}^{2}=\left\|\mathbf{k}_{\mathbf{c}}\right\|^{2}$. Die Amplitude $f_{c}^{2}$ kann analog zu dem Vorgehen aus Abschnitt 6.3 eliminiert werden, wodurch sich unter Verwendung der Abkürzung $\alpha=(a-b) /(1+a b)$ die gewöhnliche Differentialgleichung

$$
\frac{1}{\alpha} h^{\prime \prime}=\left(k_{c}+h^{\prime}\right)^{2}-\left(k_{0}^{2}+g(\mathbf{x}) \Delta k^{2}\right)
$$

für die Funktion $h(\mathbf{x})$ ergibt. $\Delta k^{2}$ ist die in Gl. (6.12) definierte Wellenzahlverstimmung. Mit $H(\mathbf{x})=k_{c}+h^{\prime}(\mathbf{x})$ kann Gl. (6.17) wiederum in die Ricatti-Differentialgleichung

$$
H^{\prime}(\mathbf{x})=\alpha H^{2}(\mathbf{x})-\alpha\left(k_{0}^{2}+g(\mathbf{x}) \Delta k^{2}\right),
$$

überführt werden, die unglücklicherweise keine allgemeine geschlossene analytische Lösung besitzt. Einen Spezialfall stellt die Bedingung $b \operatorname{Re}\left(T\left(\omega_{0}\right)\right)-\operatorname{Im}\left(T\left(\omega_{0}\right)\right)$ dar, wodurch $\Delta k=$ 0 erfüllt ist. Da in diesem Fall $k_{c}=k_{0}$ ist, ergeben sich die Lösungen der Gl. (6.17) zu

$$
\begin{aligned}
& h_{1}(\mathbf{x})=\varphi_{1} \\
& h_{2}(\mathbf{x})=-2 k_{0} \mathbf{x}+\varphi_{2}
\end{aligned}
$$

mit den konstanten Phasen $\varphi_{1}$ und $\varphi_{2}$. Die Gln. (6.19) und (6.20) führen wiederum auf die beiden ebenen Wellen

$$
f_{1}(\mathbf{x}, t)=f_{c} e^{i\left(\mathbf{k}_{\mathbf{c}} \cdot \mathbf{x}+h_{1}(\mathbf{x})-\omega_{0} t\right)}=f_{c} e^{i\left(\mathbf{k}_{0} \cdot \mathbf{x}-\omega_{0} t+\varphi_{1}\right)}
$$

und

$$
f_{2}(\mathbf{x}, t)=f_{c} e^{i\left(\mathbf{k}_{\mathbf{c}} \cdot \mathbf{x}+h_{2}(\mathbf{x})-\omega_{0} t\right)}=f_{c} e^{i\left(-\mathbf{k}_{0} \cdot \mathbf{x}-\omega_{0} t+\varphi_{2}\right)},
$$

die sich in entgegengesetzten Richtungen ausbreiten und über dieselbe Frequenz und Wellenzahl verfügen wie die freilaufende instabile Lösung der GLE (6.5). Lediglich die Amplitude $f_{c}=\sqrt{f_{0}^{2}+g(\mathbf{x}) \operatorname{Re}\left(T\left(\omega_{0}\right)\right)}$ wird durch die Regelung verändert, was allerdings durch geeignete Wahl von $k_{m a}, k_{m b}$ und $\tau_{m}$ minimierbar ist.

Um Gl. (6.17) für $\Delta k \neq 0$ nummerisch zu integrieren, sei an dieser Stelle der eindimensionale Fall betrachtet. Für eine sinusförmige Modulation $g(x)=\left(1+\sin \left(k_{g} x\right)\right) / 2$ mit der Wellenzahl $k_{g}=0.9$ besitzt die Lösung $h(x)$ der Differentialgleichung (6.17) den in Abb. 6.4b gezeigten nahezu harmonischen Verlauf. Wird daher umgekehrt von einer gegebenen harmonischen Phasenfunktion $h(x)=\epsilon \sin \left(k_{p} x+\varphi\right)$ mit Amplitude $\epsilon$, Phase $\varphi$ und 

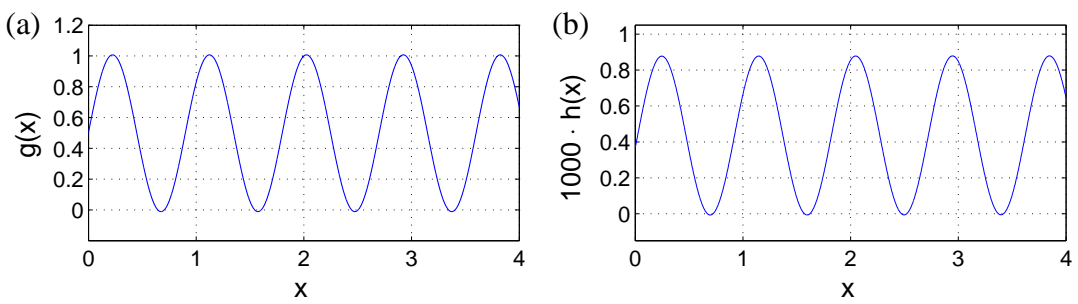

Abb. 6.4: (a) Eindimensionale sinusförmige Modulation $g(x)=\left(1+\sin \left(k_{g} x\right)\right) / 2$ mit $k_{g}=0.9$. (b) Nummerische (eindimensionale) Integration der Gl. (6.17). Die Phasenfunktion $h(x)$ zeigt durch die Modulation $g(x)$ ebenfalls nahezu harmonisches Verhalten. Die verwendeten Parameter lauten: $k_{0}=0.9, \Delta k=0.32$. Die Regelung $u(t) \neq 0$ geht ausschließlich durch $\Delta k$ in die Berechnungen ein.

Wellenzahl $k_{p}$ ausgegangen, kann die analytische (eindimensionale) Lösung der Gl. (6.17) $\mathrm{zu}$

$$
g(x)=\gamma+\delta\left(\epsilon^{2} \cos \left(2 k_{p} x+2 \varphi\right)+\frac{2 \epsilon}{\alpha} \sin \left(k_{p} x+\varphi\right)+4 \epsilon k_{c} \cos \left(k_{p} x+\varphi\right)\right)
$$

mit $\gamma=\left(2 k_{c}^{2}+\epsilon k_{p}^{2}-k_{0}^{2}\right) /\left(2 \Delta k^{2}\right)$ und $\delta=k_{p}^{2} /\left(2 \Delta k^{2}\right)$ berechnet werden. Dabei treten höhere Harmonische mit der doppelten Wellenzahl $2 k_{p}$ auf, die allerdings für kleine Amplituden $\epsilon$ nur geringen Einfluss auf $g(x)$ besitzen.

\subsection{Lokale Rückkopplung}

Die in Abschnitt 6.3 betrachtete homogene Rückkopplung ist vom theoretischen Standpunkt aus interessant, da mit ihrer Hilfe in bestimmten Fällen noch analytische Lösungen zu berechnen sind. In der Praxis ist es allerdings sehr schwierig, eine Verzögerungsregelung zu implementieren, bei der Signale homogen gemessen und dann an allen Punkten des betrachteten Gebietes angewendet werden können. Im Gegensatz zu dem durch Gl. (6.5) mit $g(\mathbf{x})=1$ und Gl. (6.7) beschriebenen idealisierten Fall besitzt jeder experimentelle Sensor eine bestimmte Ausdehnung und wird die Aktivität des interessierenden Prozesses in der gemittelten Form $\int_{S_{j}(\mathbf{x})} f(\mathbf{z}, t)$ d $\mathbf{z}$ innerhalb der Sensorregion $S_{j}(\mathbf{x})$ messen [69], die am Ort $\mathbf{x}$ zentriert sei. Normalerweise werden nur Sensoren derselben Größe verwendet, wodurch sich $S_{j}(\mathbf{x})=S(\mathbf{x})$ ergibt. Diese Messsignale werden anschließend nicht mehr homogen, sondern nur noch an ausgewählten Kontrollzellen $\mathcal{C}_{j}$ wieder auf das dynamische System zurückgekoppelt, deren Anordnung innerhalb des dynamischen Gebietes durch die räumliche Funktion $g(\mathbf{x})$ beschrieben werden kann. Dabei wird $g(\mathbf{x})$ als Weiterentwicklung der im Abschnitt 6.4 diskutierten sinusförmigen Modulation einen rechteckförmigen 
Abb. 6.5: Schematische Darstellung des in Zellen unterteilten Integrationsgebietes. Die weiss gekennzeichneten Kontrollzellen, die die Dynamik in ihrem Gebiet mitteln, haben die Abmessungen $a_{x}, a_{y}$ sowie die horizontalen bzw. vertikalen Abstände $d_{x}, d_{y}$ voneinander. Neben dem Messen der Dynamik dienen die Kontrollzellen zudem der Anwendung eines Regelsignals.

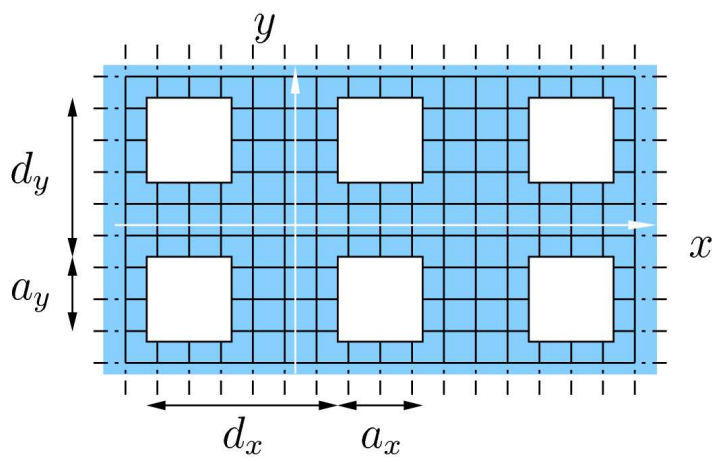

Verlauf aufweisen, wodurch das Kontrollsignal

$$
u(\mathbf{x}, t)=\sum_{m=1}^{M} k_{m a} \int_{\mathcal{C}_{j}} f\left(\mathbf{z}, t-\tau_{m}\right) \mathrm{d} \mathbf{z}-k_{m b} \int_{\mathcal{C}_{j}} f(\mathbf{z}, t) \mathrm{d} \mathbf{z},
$$

nur am Ort der Kontrollzelle $\mathcal{C}_{j}$ aktiviert wird. Wieder bezeichnen $k_{m a}, k_{m b}$ Verstärkungen und $\tau_{m}$ die von MDFC verwendeten Verzögerungszeiten. Bei Betrachtung ebener Wellen (6.15) und kleiner Sensorregionen mit einem Durchmesser $D<2 \pi / k_{c}$ vereinfacht sich Gl. (6.24) zu

$$
u(\mathbf{x}, t)=c\left(\sum_{m=1}^{M} k_{m a} e^{-i \omega_{0} \tau_{m}}-k_{m b}\right) f(\mathbf{x}, t)=c T\left(\omega_{0}\right) f(\mathbf{x}, t),
$$

wobei die Konstante $c$ aus der räumlichen Mittelung des Sensors resultiert. Für viele Anwendungen ist es wünschenswert, nur sehr wenige Kontrollzellen einzusetzen. Wenn aber zu wenige und auch räumlich weit voneinander getrennte Kontrollzellen verwendet werden, tritt ein ähnliches Phänomen wie bei der gewöhnlichen Beugung von Wellen auf. In diesem Fall winden sich die betrachteten Spiralwellen um die Kontrollzellen herum, ohne nachhaltig beeinflusst worden zu sein. Für die Stabilisierung von ebenen Wellen (mit Wellenvektor $\mathbf{k}_{\mathbf{c}}$ ) erscheint eine periodische Anordnung der Kontrollzellen sinnvoll, um die Dynamik phasenrichtig zu unterstützen bzw. zu regeln. Da die Ausdehnung $a_{x}, a_{y}$ der Kontrollzellen klein im Vergleich zur Wellenlänge der ebenen Welle sein soll, wird nur lokal auf die Dynamik Einfluss genommen. Dieses lokale Aktivieren des Kontrollsignals hat genau wie die in Abschnitt 6.4 behandelte Modulation eine zusätzliche Verstimmung $h(\mathbf{x})$ des Wellenvektors $\mathbf{k}_{c}$ der Welle (6.15) zur Folge. Analoges Vorgehen zum Abschnitt 6.4 liefert nun im Gegensatz zu Gl. (6.17) die Differentialgleichung

$$
\frac{1}{\alpha} h^{\prime \prime}=\left(k_{c}+h^{\prime}\right)^{2}-\left(k_{0}^{2}+c g(\mathbf{x}) \Delta k^{2}\right) .
$$

Neben $\alpha=(a-b) /(1+a b)$ bezeichnet $\Delta k^{2}$ die Wellenzahlverstimmung (6.12). Mit $H(\mathbf{x})=$ $k_{c}+h^{\prime}(\mathbf{x})$ lässt sich Gl. (6.26) in die Ricatti-Differentialgleichung

$$
H^{\prime}(\mathbf{x})=\alpha H^{2}(\mathbf{x})-\alpha\left(k_{0}^{2}+c g(\mathbf{x}) \Delta k^{2}\right)
$$


überführen. Für $g(\mathbf{x})$ soll im folgenden die periodische, rechteckförmige Funktion

$$
g(\mathbf{x})=\operatorname{rect}\left(\mathbf{x}, d_{x, y}, a_{x, y}\right)
$$

mit der Periodizität $d_{x, y}$ für die beiden Raumrichtungen $x, y$ angenommen werden. Als Amplitude stehen lediglich die Werte 0 und 1 zur Verfügung. Dabei bestimmen $a_{x, y}$ die Breiten der Abschnitte, in denen für die jeweilige Raumrichtung $g(\mathbf{x})=1$ erfüllt ist, wenn das Regelsignal aktiviert wird. Analog zu Abschnitt 6.4 soll an dieser Stelle zunächst nur der eindimensionale Fall der Gln. (6.26) und (6.28) betrachtet werden. Abb. 6.6 zeigt die daraus resultierenden, nummerisch ermittelten Lösungen der Gl. (6.26). Dabei ist zu

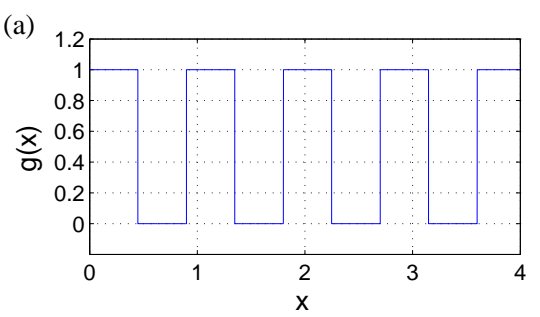

(c)

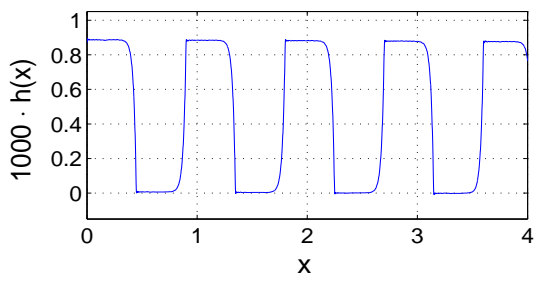

(b)

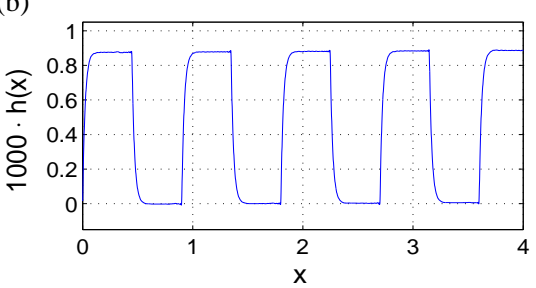

(d)

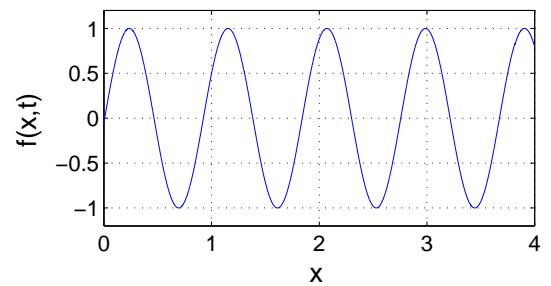

Abb. 6.6: Die (a) eindimensionale räumliche Modulation $g(x)$ führt je nach Laufrichtung der ebenen Wellen zu den örtlichen Verläufen für $h(x)$ (6.15) in (b) und (c). Für die Parameter $c=0.2, \quad k_{0}=$ 0.9 und $\Delta k=0.32$ ist deren (d) Auswirkung auf die ebenen Wellen (6.15) gering.

erkennen, dass die Anpassung der Wellenzahl an den Übergängen zwischen den Bereichen mit und denen ohne Kontrollzelle keineswegs instantan vonstatten geht, sondern Verläufe zeigt, die Lade- und Entladevorgängen von Kondensatoren ähnlich sind. Zur dauerhaften Stabilisierung ebener Wellen muss diese durch die Aktivierung der Regelung notwendige Anpassung der Wellenzahl phasenrichtig erfolgen. Um unter diesem Gesichtspunkt die Lage der Kontrollzellen zu bestimmen, wird das Rechtecksignal aus Gl. (6.28) (in jeder Raumrichtung) durch die eindimensionale Fourier-Entwicklung

$$
\operatorname{rect}\left(x, d_{x}, a_{x}\right)=\frac{a_{x}}{d_{x}}+\frac{2}{k_{c} d_{x}} \sum_{l=1}^{\infty} \sin \left(l k_{c} a_{x}\right) \frac{\cos \left(l k_{c} x\right)}{l}+\left(1-\cos \left(l k_{c} a_{x}\right)\right) \frac{\sin \left(l k_{c} x\right)}{l}
$$

approximiert. Die fundamentale Funktion $(l=1)$ der Gl. (6.29) ist $\sin \left(k_{c} x\right)$ mit der Wellenlänge $\lambda_{c}=2 \pi / k_{c}$. Wird $\lambda_{c}$ mit der (räumlichen) Korrelationslänge $C_{x y}$ der auftretenden Wellen identifiziert, erhält man mit $n \in \mathbb{N}$ die Periodizität $d_{x}=n C_{x y}$, was für $n=1$ dem Abstand benachbarter Maxima von $\sin \left(k_{c} x\right)$ entspricht. Wird der Abstand benachbarter Maxima und Minima von $\sin \left(k_{c} x\right)$ ermittelt, verändert sich die Periodizität zu $d_{x}=n C_{x y} / 2$. Da die stabilisierten bzw. generierten Wellen symmetrisch in ihrer räumlichen und zeitlichen Entwicklung sein sollen, sind die Perioden phasenrichtiger und 
phasenfalscher Rückkopplung für einen festen Ort gleich lang. Von daher ist die Bedingung $d_{x} / a_{x} \in \mathbb{Z}^{+}$erfüllt, wodurch die maximale Ausdehnung der Kontrollzellen $a_{x}=d_{x} /(2 n)$ beträgt, wenn zusätzlich die Bedingung $D<2 \pi / k_{c}$ berücksichtigt wird. $D$ bezeichnet in diesem Zusammenhang den Durchmesser der Kontrollzellen.

Zur weiteren Illustration dieser theoretischen Ergebnisse werde ich im folgenden verschiedene zweidimensionale Beispiele präsentieren, in denen die Dynamik der GLE (6.5) durch nur wenige Kontrollzellen hinsichtlich einer bestimmten Zieldynamik manipuliert wird. Die Maße der Kontrollzellen sind für diesen Zweck zu $a_{x}=C_{x y} / 4$ und $a_{y}=C_{x y} / 2$ gewählt. Bei dem betrachteten dynamischen Gebiet handelt es sich um ein $90 \times 90$ Zellen umfassendes Gitter $(\Delta x=\Delta y=1)$ auf dem die GLE (6.5) mit periodischen Randbedingungen nummerisch integriert wird.

Im ersten Beispiel handelt es sich um die Umwandlung turbulenter Dynamik (GLEParameter $a=1.1, b=-1$, Abb. 6.7a) in laufende ebene Wellen. Zu diesem Zweck wird MDFC mit dem in Abb. 6.7g angegebenen Kopplungsschema gemäß der Regelgleichung

$$
u_{j}(t)=\sum_{m=1}^{3} k_{m a} f\left(\mathcal{C}_{j}, t-\tau_{m}\right)-k_{m b} f\left(\mathcal{C}_{j}, t\right)
$$

für die $j$-te Kontrollzelle $\mathcal{C}_{j}$ (mit den gemittelten Werten $f\left(\mathcal{C}_{j}, t\right), f\left(\mathcal{C}_{j}, t-\tau_{m}\right)$ ) und den Parametern $\tau_{1}=25, \tau_{2}=62, \tau_{3}=94, k_{1 a}=0.33, k_{1 b}=0.67, k_{2 a}=0.365, k_{2 b}=0.68$, $k_{3 a}=0.405$ und $k_{3 b}=0$ angewendet, die nach Ablauf der Transienten (Abb. 6.7b) laufende ebene Wellen im Bereich zwischen den weiss markierten Kontrollzellen nach sich ziehen. Dabei sei an dieser Stelle darauf hingewiesen, dass so ein Ergebnis wegen der geltenden Torsionsbeschränkung $a b<-1$ mit (homogenem) (E)TDAS nicht möglich ist [55]. Werden die Parameter $a, b$ aus Gl. (6.5) zu $a=-1.45, b=0.34$ verändert, so entwickelt sich ausgedehnte Spiraldynamik bei abgeschalteter Regelung (Abb. 6.7d), die ebenso mit Hilfe von MDFC in ebene Wellen überführt werden kann. Erneute Anwendung des Kopplungsschemas aus Abb. 6.7g resultiert zusammen mit den Regelparametern $\tau_{1}=20, \tau_{2}=59$, $\tau_{3}=94, k_{1 a}=0.13, k_{1 b}=0.43, k_{2 a}=0.4, k_{2 b}=0.49$ in den laufenden ebenen Wellen, die in Abb. 6.7f zu erkennen sind. Um die zeitliche Dynamik zu visualisieren, zeigt Abb. 6.7h die Phasenwerte als Funktion der Zeit in einem vertikalen Schnitt durch die $x$ - $y$-Ebene an der Stelle $x=-10$. Nach der ersten Aktivierung des Parametersatzes P mit $\tau_{1}=20$, $\tau_{2}=59, \tau_{3}=104, k_{1 a}=0.13, k_{1 b}=0.43, k_{2 a}=0.4, k_{2 b}=0.49, k_{3 a}=0, k_{3 b}=0$ zur Zeit $t=300$ werden die zwischen den Reihen der Kontrollzellen auftretenden Spiralwellen in ihren Wellenfronten begradigt und diese dann auf ihre endgültige (konstante) Geschwindigkeit beschleunigt. Für $t>700$ wird schließlich anstelle der Parameter aus $\mathrm{P}$ der Kontrollparametersatz $\mathrm{S}$ mit $\tau_{1}=20, \tau_{2}=59, \tau_{3}=104, k_{1 a}=0.23, k_{1 b}=0.43$, $k_{2 a}=0.32, k_{2 b}=0.21, k_{3 a}=0.1, k_{3 b}=0$ aktiviert. Es sei an dieser Stelle darauf hingewiesen, dass sich beide Parametersätze nur durch die Vorfaktoren $k_{m a}, k_{m b}$ unterscheiden, was allerdings in einer deutlich veränderten (in diesem Fall verringerten, konstanten) Geschwindigkeit der ebenen Wellen resultiert und zu einer um den Faktor Zehn größeren 

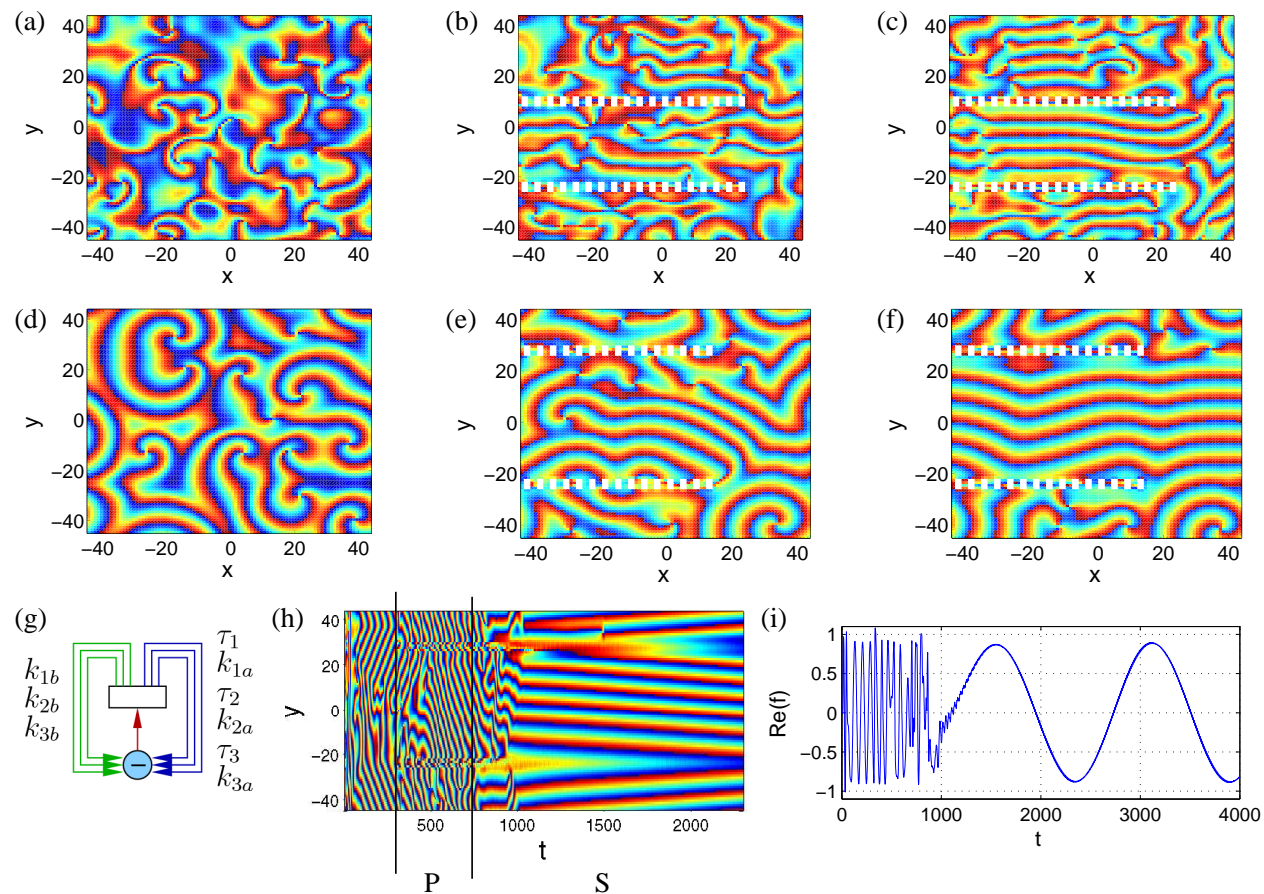

\begin{abstract}
Abb. 6.7: Farbkodierte Darstellung der raum-zeitlichen Entwicklung der Phase der komplexen Lösungsfunktion der zweidimensionalen GLE (6.5) für $a=1.1, b=-1$ (a-c) sowie $a=-1.45, b=0.34$ (d-f). Ohne Regelung ist turbulente (a) oder Spiraldynamik (d) zu erkennen. Mit an den Kontrollzellen (weiss markiert) angewendetem MDFC entsprechend Gl. (6.30) ist transiente Dynamik feststellbar (b),(e), nach deren Ablauf als asymptotische Dynamik ebene Wellen auftreten, die sich mit konstanter Geschwindigkeit ausbreiten (c),(f). Hierbei werden verzögerte und unverzögerte Signale für jede Kontrollzelle entsprechend dem Schema aus (g) angewendet. (h) Raum-zeitliche Entwicklung der Dynamik innerhalb eines vertikalen Schnittes durch (f) an der Stelle $x=-10$. Das Regelsignal mit dem Parametersatz P wird für $t>300$ aktiviert und für $t>700 \mathrm{zu}$ Gunsten des Parametersatzes S verändert, was in einer verringerten Geschwindigkeit und einer längeren Frequenz (i) für $\operatorname{Re}(f)$ der ebenen Wellen am Punkt (-35,9) resultiert. Alle Parametersätze sind dem Text zu entnehmen.
\end{abstract}

Wellenlänge führt (Abb. 6.7i). So ist neben der Wahl einer endlichen Geschwindigkeit im Extremfall ein (zeitlich begrenztes, ortsfestes) Einfrieren der Wellen oder aber eine deutliche Veränderung der Wellenlänge möglich, was für viele Anwendungen wie z. B. die Kontrolle von Herz-Rhythmus-Störungen ${ }^{1}$ wichtig und durch den invasiven Charakter

\footnotetext{
${ }^{1}$ Häufig wird für die Simulation dieser besonderen Herzdynamik das bekannte Fitzhugh-NagumoSystem (FHS) verwendet. Motiviert durch die Erfolge von MDFC bei der GLE (6.5) habe ich sowohl NFF als auch MDFC am FHS ausprobiert. Dabei hat sich herausgestellt, dass mit beiden Methoden eine Stabilisierung von ebenen Wellen möglich ist. Um den Gedankenfluss bezüglich der GLE nicht zu
} 
von MDFC bei entsprechender Parameterwahl bedingt ist. Simulationen haben gezeigt,

Abb. 6.8: Farbkodierte Darstellung der raum-zeitlichen Entwicklung der Phase der GLE (6.5) ausgehend vom freilaufenden System aus Abb. 6.7d. (a) Erzeugung zweier kontrarotierender Spiralwellen während der transienten Phase, deren Dynamik von einer Art (b) Wellenbaseball abgelöst wird. Angewendet wird MDFC (6.31) und die Kopplung aus (d). Die Kontrollzellen sind in Liniensegmenten zusammengefasst. (c) Detaillierte Darstellung der Kopplungen aus (d) für den umrandeten Bereich.
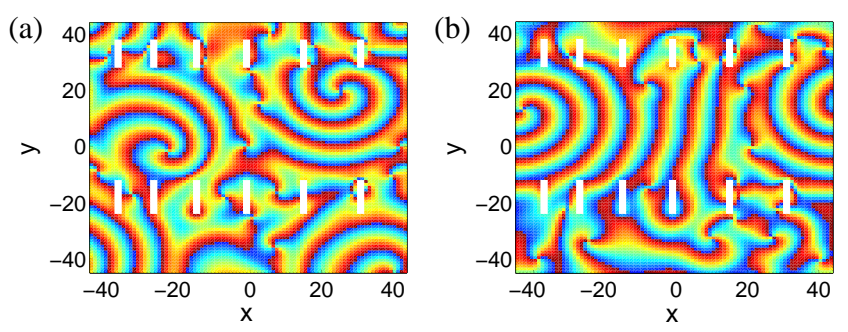

(c)

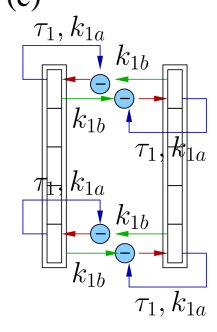

(d)

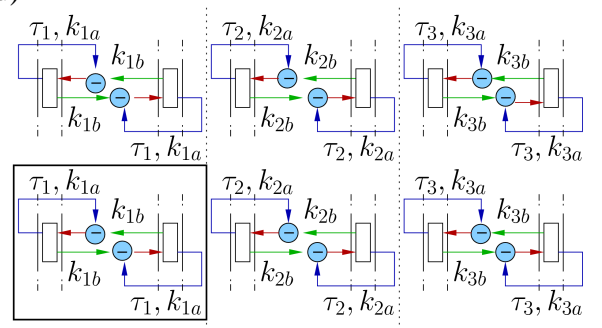

dass für diesen Zweck der horizontale Abstand der wenigen eingesetzten Kontrollzellen die räumliche Korrelationslänge $C_{x y}$ nicht überschreiten sollte. Die beste Wirkungsweise wird dabei erzielt, wenn die zu Anfang angegebene Dimensionierung der Kontrollzellen gewählt und deren Abstand auf die Hälfte der räumlichen Korrelationslänge gesetzt wird. Die Verstärkungen werden experimentell eingestellt (ausgehend vom symmetrischen Fall $\left.k_{m a}=k_{m b}\right)$. Von den drei Verzögerungszeiten sind für diese Zieldynamik zwei in der Nähe von Nullstellen und eine im Bereich eines Maximums der zeitlichen Autokorrelationsfunktion von $f$ aus Gl. (6.5) platziert. Um ebene Wellen in bestimmte ausgewählte Raumrichtungen (Sektoren) zu lenken, muss die oben beschriebene Regelgeometrie zu Gunsten eines verschobenen Xs geändert werden.

Aber nicht nur die Erzeugung von ebenen Wellen steht im Fokus dieser Untersuchungen. In den vorangegangenen Beispielen wurde für jede Kontrollzelle ein individuelles Regelsignal generiert, das nur die Dynamik der betrachteten Kontrollzelle berücksichtigt und schließlich wieder auf diese zurückkoppelt (Abb. 6.7d). Das ist selbstverständlich nicht die einzige Möglichkeit, wie MDFC bei räumlich ausgedehnten Systemen appliziert werden kann. Im allgemeinen kann die gemessene Dynamik verschiedener Zellen miteinander kombiniert und das resultierende Kontrollsignal an anderer Stelle eingespeist werden. Zwei dieser nicht mehr trivialen Rückkopplungen liefern die Abb. 6.8 und 6.9. Die Parameter $a=-1.45$ und $b=0.34$ der Ginzburg-Landau Gleichung (6.5) sind so gewählt wie in Abb. 6.7d-f, was in chaotischer Spiraldynamik resultiert, wenn die Regelung nicht angeschaltet wird (Abb. 6.9a). Die Kontrollzellen werden dabei jeweils in Liniensegmenten

zerstören, sei an dieser Stelle lediglich auf die entsprechenden Simulationsergebnisse im Anhang ab Seite 127 verwiesen. 
zusammengefasst, wie Abb. $6.8 \mathrm{c}$ zu entnehmen ist. Paare von gegenüberliegenden Liniensegmenten ${ }^{2}$ sind dabei sowohl räumlich als auch zeitlich durch das Regelsignal
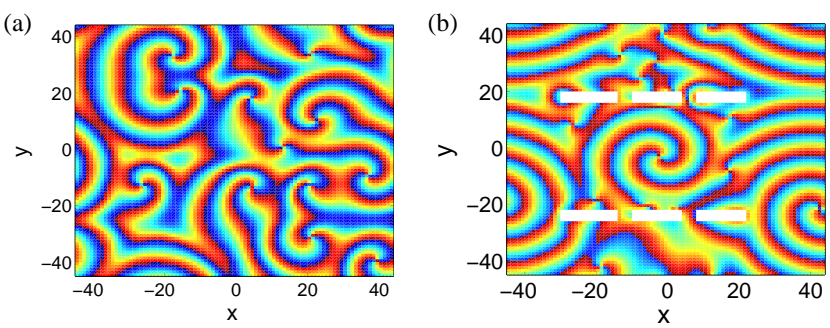

(c)

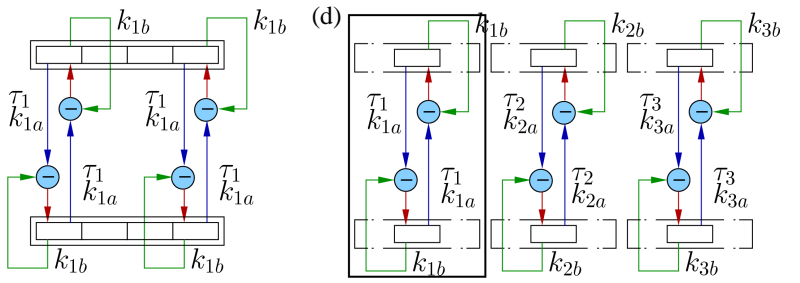

Abb. 6.9: Farbkodierte Darstellung der raum-zeitlichen Entwicklung der Phase der komplexen GinzburgLandau-Gleichung (6.5). (a) Freilaufendes System. (b) Generieren und Fangen einer Spiralwelle innerhalb des Regelgebietes unter Zuhilfenahme des Kopplungsschemas aus (d) und MDFC (6.32). (c) Detaillierte Ansicht der räumlichen und zeitlichen Kopplungen gegenüberliegender Kontrollzellen aus dem umrandeten Bereich aus (d).

$$
u_{p r}(t)=k_{m a} f\left(\mathcal{C}_{p l}, t-\tau_{m}\right)-k_{m b} f\left(\mathcal{C}_{p r}, t\right)
$$

für die Kontrollzellen der rechten (Index $r$ ) bzw. durch

$$
u_{p l}(t)=k_{m a} f\left(\mathcal{C}_{p r}, t-\tau_{m}\right)-k_{m b} f\left(\mathcal{C}_{p l}, t\right)
$$

für die entsprechenden Kontrollzellen der linken Seite (Index $l$ ) miteinander verbunden. Die Verzögerungszeiten $\tau_{m}$ sowie die Verstärkungsfaktoren $k_{m a}$ und $k_{m b}$ sind für je ein Paar der Anordnung aus Abb. 6.8c identisch, können aber von denen der anderen Paare aus Abb. 6.8d abweichen. Mit den aktivierten Kontrollparametern $k_{1 a}=0.11, k_{2 a}=0.25$, $k_{3 a}=0.32, k_{1 b}=0.27, k_{2 b}=0.2, k_{3 b}=0.39, \tau_{1}=57, \tau_{2}=29$ und $\tau_{3}=79$ treten während der transienten Phase (Abb. 6.8a) zwei gegeneinander rotierende Spiralwellen auf. Diese Dynamik stellt sich allerdings als instabil heraus, so dass aufgrund des Wettbewerbprozesses der Spiralwellen nur eine Spirale, d. h. eine Drehrichtung weiter bestehen kann. Das asymptotische Verhalten aus Abb. 6.8b ähnelt einer Art Wellen-Baseball, bei dem von einer Spiralwelle (Werfer) ausgesandte Wellenfronten durch die Regelung begradigt und von der nächsten Spiralwelle wieder aufgefangen werden (Fänger) ${ }^{3}$.

Um eine der in der transienten Phase (Abb. 6.8a) auftretenden Spiralwellen zu stabilisieren, muss die Kontrollanordnung aus Abb. 6.8d zugunsten der in Abb. 6.9d gezeigten modifiziert werden. Die drei Paare gegenüberliegender Liniensegmente bestehen jeweils

\footnotetext{
${ }^{2} \mathrm{Im}$ folgenden verfügen Kontrollzellen in gegenüberliegenden Liniensegmenten immer über denselben Index $p$.

${ }^{3}$ Wegen der periodischen Randbedingungen sind die emittierende und die fangende Spiralwelle identisch.
} 
aus mehreren Kontrollzellen (Abb. 6.9c). Vergleichbar zu Abb. 6.8c besitzt wieder jedes dieser Paare von Liniensegmenten seine eigene Verzögerungszeit und geeignete Verstärkungsfaktoren wie Abb. 6.9c zu entnehmen ist. Unter Verwendung der Regelgleichungen

$$
u_{p u}(t)=k_{m a} f\left(\mathcal{C}_{p o}, t-\tau_{m}\right)-k_{m b} f\left(\mathcal{C}_{p u}, t\right)
$$

für die Kontrollzellen des unteren (Index $u$ ) bzw.

$$
u_{p o}(t)=k_{m a} f\left(\mathcal{C}_{p u}, t-\tau_{m}\right)-k_{m b} f\left(\mathcal{C}_{p o}, t\right)
$$

für die Kontrollzellen des (gegenüberliegenden) oberen Liniensegmentes (Index o) wird eine rotierende Spiralwelle nach Ablauf von Transienten innerhalb der Regelgeometrie gefangen und stabilisiert (Abb. 6.9b). Die hierzu verwendeten Parameter lauten $k_{1 a}=0.22$, $k_{2 a}=0.1, k_{3 a}=0.35, k_{1 b}=0.3, k_{2 b}=0.5, k_{3 b}=0.0, \tau_{1}=41, \tau_{2}=27$ und $\tau_{3}=49$. Die

Abb. 6.10: Entwicklung der Phase der GLE (6.5). Das Kopplungsschema aus (b) erzeugt ebene, gescherte Wellen, die sich mit konstanter Geschwindigkeit von links nach rechts bewegen (a).
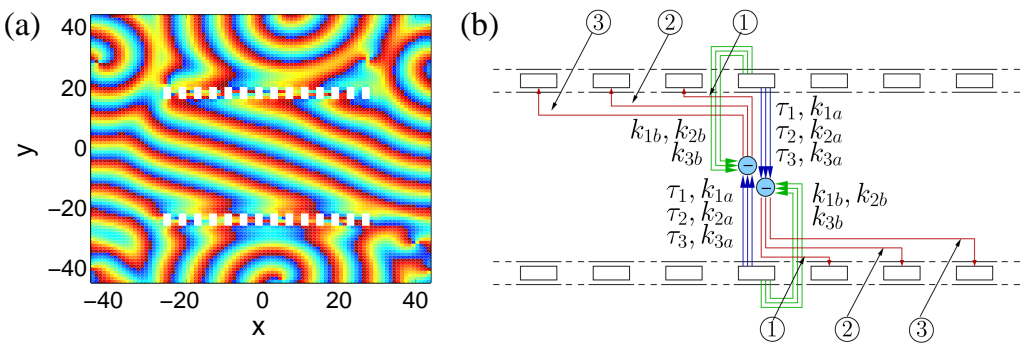

Rotationsrichtung der gefangenen Spirale hängt von den Rückkopplungsparametern und dem vertikalen Abstand der Kontrollzellen ab, der geeignet gewählt sein sollte. Ist der Abstand zu gering oder zu groß, hat die Spiralwelle entweder nicht genügend Platz, um sich auszubilden oder aber komplexe Spiraldynamik mit mehreren Spiralwellen bzw. andere turbulente Strukturen werden generiert.

Das letzte illustrative Beispiel für die Vielfältigkeit der Anwendungen von MDFC ist der Abb. $6.10 \mathrm{zu}$ entnehmen, wodurch sich demonstrieren lässt, wie aus Spiralwellen laufende, schräge Wellen generiert werden können. Wieder wird mit den Parametern $a=-1.45$ und $b=0.34$ aus Gl. (6.5) gestartet, wobei das Kopplungsschema aus Abb. 6.10b zum Einsatz kommt. Das Ergebnis der mittels der Regelgleichung

$$
u_{Y_{m} u}(t)=\sum_{m=1}^{3} k_{m a} f\left(\mathcal{C}_{p o}, t-\tau_{m}\right)-k_{m b} f\left(\mathcal{C}_{p u}, t\right)
$$

für die unteren bzw.

$$
u_{Z_{m} o}(t)=\sum_{m=1}^{3} k_{m a} f\left(\mathcal{C}_{p u}, t-\tau_{m}\right)-k_{m b} f\left(\mathcal{C}_{p o}, t\right)
$$


für die oberen Kontrollzellen eingesetzten Parameterkombination $\tau_{1}=31, \tau_{2}=59$, $\tau_{3}=84, k_{1 a}=0.22, k_{1 b}=0.3, k_{2 a}=0.2, k_{2 b}=0.5, k_{3 a}=0.3, k_{3 b}=0.0 \mathrm{kann}$ in Abb. 6.10b gesehen werden. Dabei ist für $n$ Kontrollzellen pro horizontalem Liniensegment $Y_{m}=\left(p+d_{m}\right) \bmod (n)$ bzw. $Z_{m}=\left(p-d_{m}\right) \bmod (n)$ der seitliche Versatz an Kontrollzellen, an denen die gemessenen Signale wieder auf die GLE (6.5) wirken. Wie weit die Wellenfronten letztendlich geschert werden, hängt von den Verschiebungsparametern $d_{m}$ für die Kontrollzelle $\mathcal{C}_{j}$ ab.

(a)

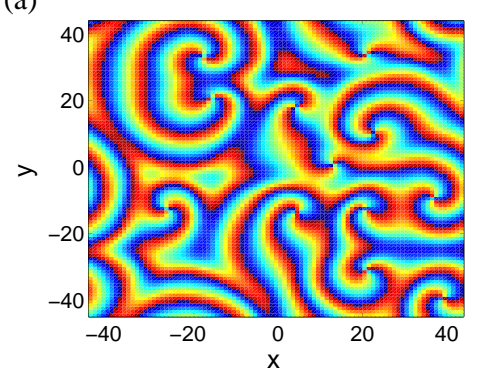

(c)

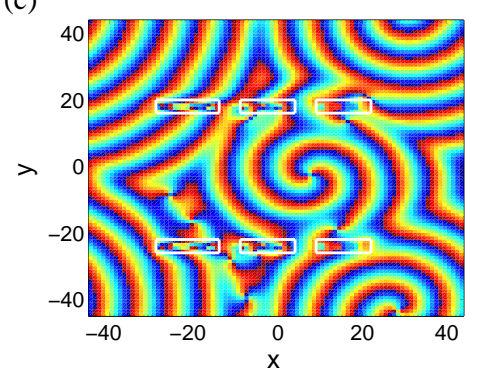

(b)

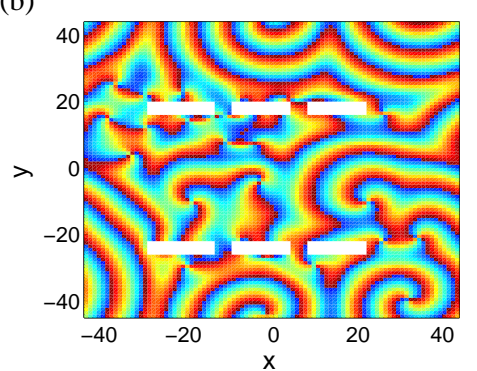

(d)

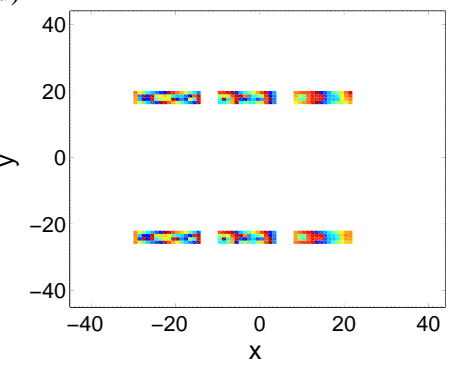

Abb. 6.11: Raum-zeitliche Entwicklung der Phase der komplexen GLE (6.5). (a) Generieren und (c) Einfangen einer Spiralwelle mit MDFC (6.32) gemäß des Kopplungsschemas aus Abb. 6.9c,d nach Ablauf der Transienten (b). Der Bereich der Kontrollzellen ist weiss markiert und in (d) unter Weglassen der äußeren Dynamik detailliert dargestellt. Feststellbar ist eine synchronisierte Dynamik zwischen oberer und unterer Aktuatorreihe.

Aber das ist nicht der einzige Aspekt, der an dieser Stelle behandelt werden kann. Zu diesem Zweck muss wieder auf das Beispiel der Spiralfalle zurückgegeriffen werden. Bisher stand immer nur die Dynamik zwischen den Kontrollzellen im Fokus der Untersuchungen. Erweitert man diese Analyse auf die Kontrollzellen der Abb. 6.11, so ist klar eine Synchronisation der Dynamik der oberen und der unteren Kontrollzellen zu erkennen.

\subsection{Synchronisation}

Der Vorgang der Synchronisation ist bereits seit ca. 1665 bekannt. Ausgangspunkt war Huygens' Beobachtung, dass zwei miteinander gekoppelte Uhren über lange Zeit ihre Dynamik aneinander angleichen und im Gleich- oder Gegentakt arbeiten. Seitdem wurde dieser Effekt in vielen biologischen, physikalischen und chemischen Systemen nachgewiesen oder zur Anwendung gebracht [40,128, 146]. Beispiele hierfür sind der Vorgang der Epilepsie, bei dem Neuronen plötzlich miteinander synchronisieren und zu entsprechenden Muskelkontraktionen führen oder die Synchronisation (chaotischer) Systeme [39, 108, 113, 115]. 
$\mathrm{Zu}$ diesem Zweck stellt ein treibendes System ein Zeitsignal zur Verfügung, das mit Hilfe eines Kopplungsfaktors auf ein zweites (getriebenes) Antwort-System einwirkt. Diese unidirektionale Kopplung kann zu einer bidirektionalen erweitert werden, indem auch das Antwort-System seinerseits seine Dynamik dem Treiber-System zuführt. Dabei können Treiber- und Antwort-System von der Art her unterschiedlich sein, wobei allerdings die Synchronisation (d.h. die Angleichung der Dynamik beider Systeme bei geeigneter Parameterwahl) gleicher Systeme einfacher vonstatten geht. Unter diesem Gesichtspunkt kann bei zwei identischen Systemen vollständige d.h. identische Synchronisation auftreten, bei der sich (chaotische) Zustände beider Systeme identisch ändern [78]. Sollten sich die Zustandsvektoren des Treiber- und des Antwortsystems nicht identisch ändern, aber dennoch über eine bestimmte (im Einzelfall komplizierte) Funktion miteinander verknüpft sein, spricht man von verallgemeinerter Synchronisation $[2,78,134]$, die in vielen Fällen aus einer unidirektionalen Kopplung verschiedener Systeme resultiert. Ein Spezialfall der verallgemeinerten Synchronisation ist die sogenannte Lag Synchronisation [130,142].

Abb. 6.12: Identische Synchronisation der GLE (6.5) mit den Parametern $a=-1.45$ und $b=$ 0.34 für die bidirektionale Kopplung (6.34f). (a) Transiente nach Aktivierung des Signals (6.34f) mit weiss markierten Kontrollzellen, deren Verschaltung in (d) bzw. im Detail für zwei verknüpfte Kontrollzellen in (c) zu sehen ist. (b) Synchronisierte Dynamik innerhalb der weiss umrandeten Bereiche (Kontrollriegel).
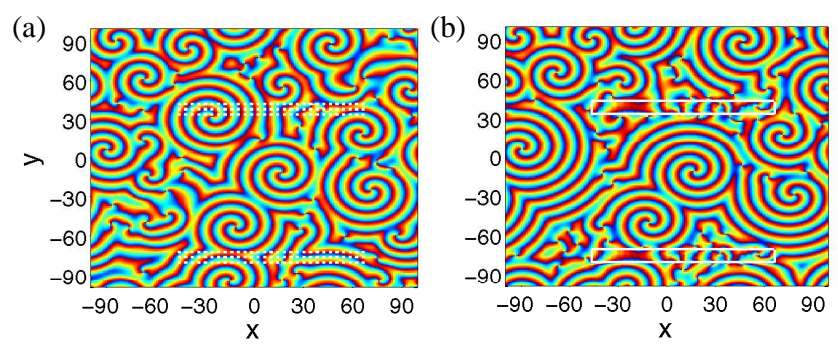

(c)

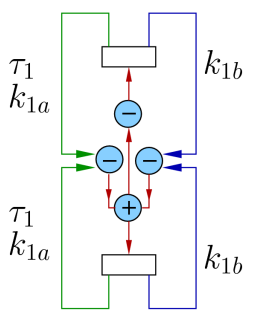

(d)

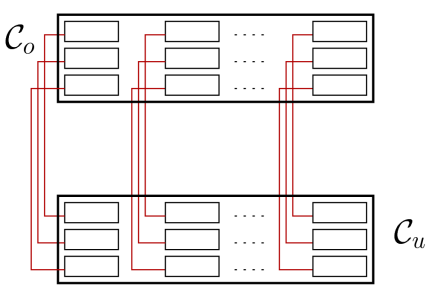

Zur Untersuchung dieser Vorgänge in räumlich ausgedehnten Systemen wird im folgenden die zweidimensionale GLE (6.5) mit den Parametern $a=-1.45, b=0.34$ dienen, wobei das Integrationsgebiet auf $200 \times 200$ Zellen vergrößert wird. Um einen großen räumlichen Abstand zu gewährleisten, erfolgt die Platzierung der Kontrollzellen in zwei horizontalen Riegeln, deren vertikaler Abstand etwa der zehnfachen räumlichen Korrelationslänge $C_{x y}$ entspricht. Innerhalb der beiden Riegel sind Kontrollzellen der Abmessungen $a_{x}=C_{x y} / 4$, $a_{y}=C_{x y} / 4$ in den horizontalen und vertikalen Abständen $d_{x}=C_{x y} / 2, d_{y}=C_{x y} / 2$ angeordnet. Da der innere Aufbau des oberen und unteren Riegels identisch ist, können die Kontrollzellen $\mathbf{x}_{o}$ des oberen mit den entsprechend gegenüber liegenden Zellen $\mathbf{x}_{u}$ des unteren Aktuatorriegels (Abb. 6.12d) wahlweise durch die Kontrollsignale

$$
u_{1, u}=k_{1 b} f\left(\mathbf{x}_{o}, t\right)
$$




$$
\begin{aligned}
u_{2, u}= & k_{1 b}\left[f\left(\mathbf{x}_{u}, t\right)-f\left(\mathbf{x}_{o}, t\right)\right] \\
u_{3, u}= & k_{1 a} f\left(\mathbf{x}_{o}, t-\tau\right) \\
u_{4, u}= & k_{1 a}\left[f\left(\mathbf{x}_{u}, t-\tau\right)-f\left(\mathbf{x}_{o}, t-\tau\right)\right] \\
u_{5, u}= & k_{1 a} f\left(\mathbf{x}_{o}, t-\tau\right)+k_{1 b} f\left(\mathbf{x}_{o}, t\right) \\
u_{6, u}= & k_{1 a}\left[f\left(\mathbf{x}_{u}, t-\tau\right)-f\left(\mathbf{x}_{o}, t-\tau\right)\right] \\
& +k_{1 a}\left[f\left(\mathbf{x}_{u}, t-\tau\right)-f\left(\mathbf{x}_{o}, t-\tau\right)\right]
\end{aligned}
$$

miteinander wechselwirken (Abb. 6.12c). In den Formeln (6.34a-f) ist lediglich eine unidirektionale Kopplung angegeben, die aber zu einer bidirektionalen ergänzt werden kann, indem die Indizes $u$ und $o$ vertauscht werden. Ein Beispiel für die bidirektionale Anwendung von Gl. (6.34f) zeigt Abb. 6.12. Zu Gunsten einer übersichtlicheren Darstellung der Dynamik sei auf die Markierung der einzelnen Kontrollzellen in Abb. 6.12a zugunsten der Einhüllenden des oberen und unteren Aktuatorriegels verzichtet (Abb. 6.12b). Dadurch wird der Blick für die innerhalb der beiden Riegel auftretende identisch synchronisierte Dynamik nach Ablauf einer kurzen Transienten freigegeben. Die weitere Analyse der notwendigen Parameter für eine erfolgreiche Synchronisation beschränkt sich o.B.d.A auf einen horizontalen Schnitt durch das Integrationsgebiet der Abb. 6.12b in Höhe der Mitte des unteren Kontrollriegels. Unter Verwendung des Synchronisationsfehlers

$$
\mathbf{e}(t)=\mathbf{f}\left(\mathbf{x}_{u}, t\right)-\mathbf{f}\left(\mathbf{x}_{o}, t\right),
$$

der ein Maß für die Synchronisation ist, zeigt Abb. 6.13 Beispiele für eine erfolgreiche Synchronisation der GLE (6.5) in zwei Dimensionen. Zum Einsatz kommt das Kontrollsignal (6.34f) mit bidirektionaler (Abb. 6.13a-c) bzw. unidirektionaler Kopplung (Abb. 6.13df), wobei der Synchronisationsfehler (6.35) farbkodiert dargestellt ist. Auf diese Weise kann den Abb. 6.13a,d entnommen werden, dass der synchronisierte Zustand für uni- und bidirektionale Kopplung bereits kurz nach dem dauerhaften Einschalten des Synchronisationssignals zur Zeit $t=30$ vorliegt, wenn der horizontale Abstand der Kontrollzellen innerhalb des unteren und oberen Riegels einen Wert von $d_{x} \approx C_{x y} / 2$ aufweist. Weiterhin bleibt festzustellen, dass gemäß Abb. 6.13b,e dieser horizontale Abstand der Kontrollzellen die räumliche Korrelationslänge von $C_{x y} \approx 8.5$ für eine erfolgreiche Synchronisation auf gesamter Breite der Riegel nicht überschreiten sollte. Die weiteren Parameter für diese Simulationen sind $d_{y} \approx C_{x y} / 2$ sowie $k_{1 a}=0.2$ und $k_{1 b}=0.3$. Die Abhängigkeit von diesen Verstärkungen $k_{1 a}, k_{1 b}$ unter Verwendung von $d_{x}=d_{y}=C_{x y} / 2$ kann den Abb. 6.13c,f für den bi- und den unidirektionalen Fall entnommen werden. Dabei ist zu bemerken, dass beide Verstärkungen des Synchronisationssignals (6.34f) simultan gemäß $k_{1 a}=2 k_{1 b} / 3$ geändert wurden. Festzuhalten bleibt, dass der für erfolgreiche Synchronisation auftretende Schwellwert für die bidirektionale und die unidirektionale Kopplung nahezu in ähnlichen Bereichen liegt ${ }^{4}$. Dieses Ergebnis hat sich auch bei den anderen Synchronisationssignalen

\footnotetext{
${ }^{4}$ Weiterhin ist zu erkennen, dass lediglich in diesem Beispiel für höhere Verstärkungen im bidirektionalen Fall noch wesentlich länger vereinzelte Synchronisationsfehler auftreten als für die entsprechende unidirektionale Kopplung.
} 

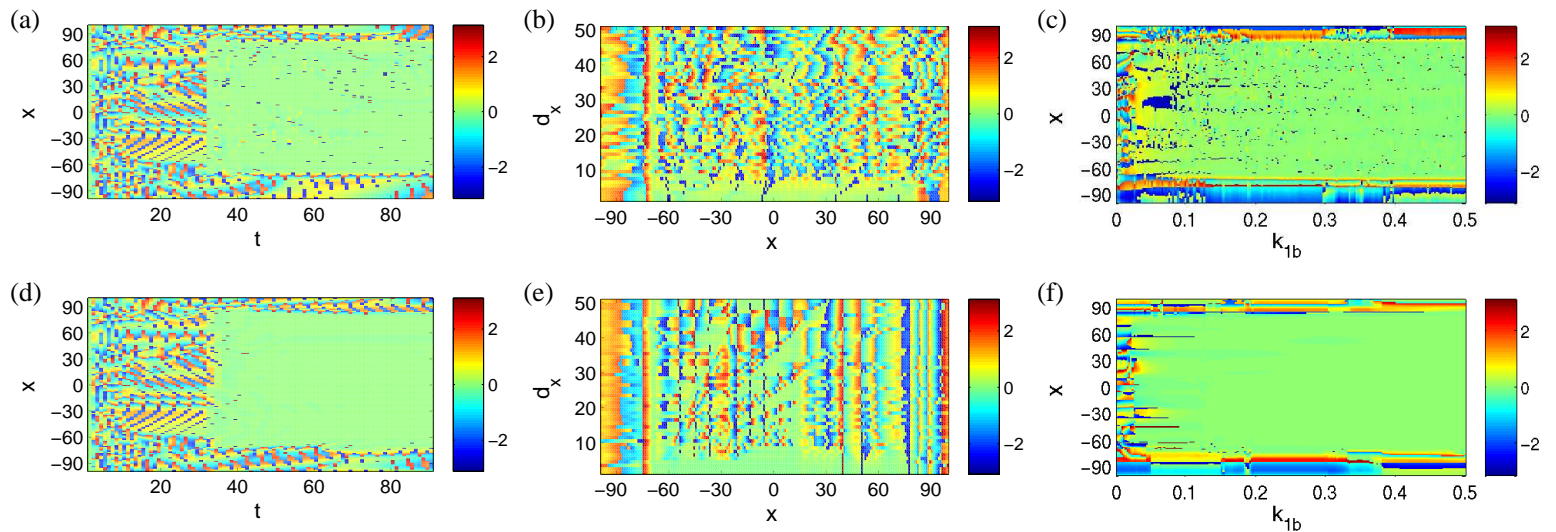

\begin{abstract}
Abb. 6.13: (a) Synchronisation der GLE (6.5) unter Verwendung des Synchronisationssignals (6.34f) für (a-c) bidirektionale Kopplung, (d-f) unidirektionale Kopplung innerhalb eines horizontalen Schnittes durch Abb. 6.12 in Höhe des unteren Kontrollriegels. Das Synchronisationssignal (6.34f) wird für $t>30$ dauerhaft aktiviert, der Synchronisationsfehler (6.35) ist farbkodiert aufgetragen. (a),(d) zeitlicher Verlauf der Synchronisation. (b),(e) Synchronisation in Abhängigkeit des horizontalen Abstandes der Kontrollzellen innerhalb der beiden Riegel. (c),(f) Einfluss der Verstärkungen $k_{1 a}, k_{1 b}$ auf die Synchronisation.
\end{abstract}

Gl. (6.34a-e) bestätigt, sofern sowohl im bi- als auch im unidirektionalen Fall Synchronisation eingetreten ist. Insgesamt ergibt sich für die verschiedenen Arten der Kopplung (6.34) das in Tab. 6.1 zusammengefasste Synchronisationsergebnis. Dabei ist erfolgreiche Synchronisation durch einen Haken gekennzeichnet. Notwendig für eine erfolgreiche Synchronisation ist die Existenz eines ausgedehnten Parameterbereiches für $k_{1 a}, k_{1 b} \in[0,1]$, in dem der Synchronisationsfehler (6.35) auf Null zurückgeht. Dieser Zusammenhang ist

Tabelle 6.1: Erfolgreiche Synchronisation mittels der Signale Gl. (6.34) für Gl. (6.5) ist durch einen Haken gekennzeichnet.

\begin{tabular}{l|c|c|c|c|c|c} 
Kopplung & $u_{1, u}$ & $u_{2, u}$ & $u_{3, u}$ & $u_{4, u}$ & $u_{5, u}$ & $u_{6, u}$ \\
\hline bidirektional & $\sqrt{ }$ & $\sqrt{ }$ & $\sqrt{ }$ & - & $\sqrt{ }$ & $\sqrt{ }$ \\
unidirektional & - & $\sqrt{ }$ & $(\sqrt{ })$ & - & - & $\sqrt{ }$
\end{tabular}

bei unidirektionaler Kopplung nur für die Gln. (6.34b) und (6.34f) erfüllt. Im Gegensatz hierzu verschwindet für Gl. (6.34c) der Synchronisationsfehler nicht, sondern nimmt einen konstanten, kleinen Wert an. Sofern nur eine einzige Verzögerungszeit ohne weiteren unverzögerten Anteil eingesetzt wird (z. B. in Gl. (6.34c)), ist eine erfolgreiche Anwendung sowohl im bi- als auch im unidirektionalen Fall an ein enges Spektrum von $\tau$ geknüpft. Unter diesem Gesichtspunkt darf die eine Verzögerungszeit $\tau$ das Zweieinhalbfache der natürlichen Periode der Oszillationen für eine erfolgreiche Synchronisation nicht über- 

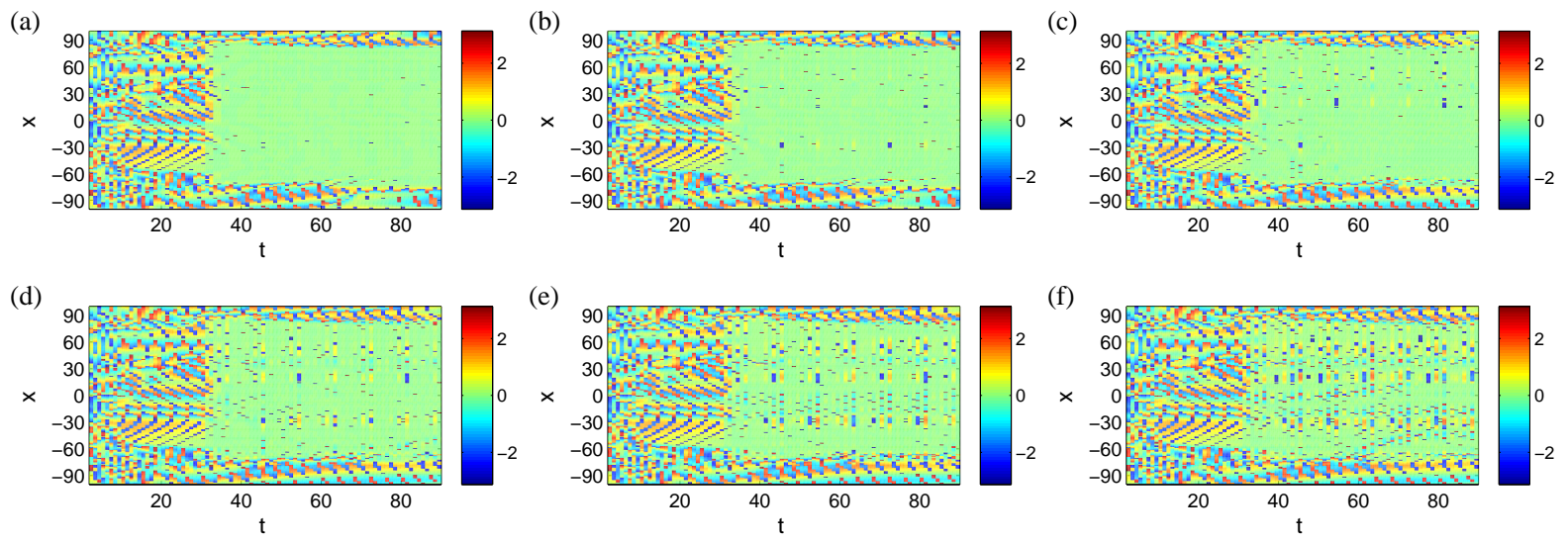

\begin{abstract}
Abb. 6.14: (a) Synchronisation für die GLE (6.5) mittels des zur Zeit $t=30$ aktivierten bidirektionalen Synchronisationssignals (6.34b). Das periodische Wegschalten dieses Signals (Periode entspricht der natürlichen Periodendauer des ungeregelten Systems) unter Verwendung des Tastverhältnisses (a) $S=0.9$, (b) $S=0.8$, (c) $S=0.7$, (d) $S=0.6$, (e) $S=0.5$, (f) $S=0.4$ resultiert in dem farbig dargestellten Synchronisationsfehler (6.35). Die Verstärkung sei zu $k_{1 b}=0.3$ gewählt.
\end{abstract}

schreiten. Weiteres Augenmerk der Untersuchungen im Hinblick auf die Robustheit der Kopplungen (6.34a-f) liegt auf dem periodischen Wegschalten des Synchronisationssignals. Dabei steht im Vordergrund, wie lange dieses Signal abgeschaltet werden kann, um dennoch dauerhaft Synchronisation zu ermöglichen und den Fehler (6.35) zu minimieren. Dieser Zusammenhang ist möglich, da das dynamische System über ein Gedächtnis verfügt. Für die technische Realisierung wird dazu das verwendete Synchronisationssignal (6.34b) mit einem zusätzlichen Rechtecksignal (Amplitude zwischen 0 und 1) multipliziert, dessen Tastverhältnis geändert werden kann. Dabei bezeichnet das Tastverhältnis $S$ das Verhältnis zwischen dem eingeschalteten Zustand und der Gesamtperiode des Rechtecksignals, die für die Simulationen auf die natürliche Schwingungsdauer der GLE (6.5) für die Parameter $a=-1.45, b=0.34$ fixiert sei. Die Ergebnisse aus Abb. 6.14 für die Tastverhältnisse (a) $S=0.9$, (b) $S=0.8$, (c) $S=0.7$, (d) $S=0.6$, (e) $S=0.5$ und (f) $S=0.4$ zeigen in diesem Zusammenhang, dass das Tastverhältnis für eine dauerhaft erfolgreiche Synchronisation bei einem horizontalen und vertikalen Abstand der Kontrollzellen von $d_{x}=d_{y} \approx C_{x y} / 2$ nicht kleiner als $S=0.9$ gewählt werden sollte, um Synchronisationsfehler stark zu begrenzen.

Generell ist festzustellen, dass bei der in Abb. 6.12 gewählten Anordnung der Kontrollzellen bei allen erfolgreichen Synchronisationssignalen (6.34a-f) eine große rotierende Spiralwelle im ausgedehnten vertikalen Bereich zwischen den Kontrollriegeln (ortsfest) stabilisiert wird. Zudem treten innerhalb dieser Region ein bis zwei weitere (kleinere) Spiralen auf, die sich innerhalb der Kontrollregion langsam bewegen. 


\section{Kapitel 7}

\section{Zusammenfassung und Ausblick}

\subsection{Zusammenfassung}

Multiple Delay Feedback Control (MDFC) ist eine effiziente Methode zur Stabilisierung von Fixpunkten, die gleichzeitig neue Möglichkeiten bei der Kontrolle und Manipulation raum-zeitlicher chaotischer Dynamik bietet. Der Hauptunterschied zu Pyragas' TDAS und seinen Erweiterungen ETDAS und NTDAS ist die Verwendung mehrerer unabhängiger Verzögerungszeiten sowie individueller Verstärkungsfaktoren zu deren Gewichtung.

Lineare Stabilitätsanalysen zeigen, dass MDFC konventionellen P- und PD-Reglern (unabhängig von einer Hochpassfilterung der Eingangssignale) sowie dem bekannten (E)TDAS überlegen ist im Hinblick auf die absoluten Stabilitätswerte und die Größe der Stabilitätsgebiete. Dabei erhöht sich die Effizienz von MDFC durch Hinzunahme weiterer individueller Verzögerungszeiten. Um diesen Gesichtspunkt besser zu verstehen, ist ein Wechsel in den Frequenzraum von Vorteil. Die Abhängigkeit der Übertragungsfunktion von den einzelnen Verzögerungszeiten und Verstärkungen bietet die Möglichkeit, MDFC sehr genau an die vorherrschende dynamische Situation anzupassen, um z. B. bestimmte störende Frequenzkomponenten zu unterdrücken. Auf diese Weise ist eine effektivere Regelung als bisher möglich, durch die Fixpunkte mit weitaus geringeren Verstärkungen stabilisiert werden können.

Lokal kann die Übertragungsfunktion von MDFC bis zu einem gewissen Grad durch eine Parallelschaltung von Kerbfiltern approximiert werden. Dieses sogenannte Notch Filter Feedback (NFF) ist ebenfalls effizient für die Stabilisierung von Fixpunkten einsetzbar. Sowohl MDFC als auch NFF sind durch ihre verhältnismäßig einfache Implementierung in Form von analoger Elektronik für die experimentelle Stabilisierung von Fixunkten bei schnellen dynamischen Systemen geeignet. Beispiele hierfür sind durch einen elektronischen Chua-Oszillator sowie einen schwer zu zähmenden kompakten intern frequenzverdoppelten Nd:YAG Laser gegeben, deren Systemfixpunkte jeweils erfolgreich experimen- 
tell stabilisiert werden konnten (Stabilisierung des Lasers auf konstante Ausgangsintensität). Gerade dieses sogenannte green problem stellt bei mittleren und hohen Pumpraten sehr hohe Anforderungen an eine Regelung, die außer von MDFC und NFF bisher durch keine andere Regelungsmethode erbracht werden konnten. Während in [138] behauptet wurde, dass die grüne Intensität als Eingangssignal einer Regelung nicht geeignet sei, konnte in dieser Arbeit das Gegenteil für MDFC und NFF gezeigt werden. Sowohl MDFC als auch NFF konnten unter Verwendung der beiden instabilen infraroten Intensitäten oder der grünen Intensität Fixpunkte erfolgreich stabilisieren. Die zusätzlich hierfür vorgenommenen Simulationen mit Hilfe eines detaillierten Lasermodells bestätigen die experimentellen Befunde. Zudem ist MDFC in Simulationen sogar noch bei einer von außen zusätzlich angewendeten sinusförmigen Modulation erfolgreich und verhindert ein erneutes Abdriften der Laserdynamik in den Chaosbereich. Vielmehr folgt die Laserintensität dem von außen aufgeprägten Modulationssignal. Ermöglicht wird dies durch die Existenz ausgedehnter Stabilitätsgebiete, in denen MDFC erfolgreich zu einer Stabilisierung des Lasers führt. Die Tatsache, dass unsymmetrische Vorfaktoren der einzelnen Rückkopplungsterme nicht nur erlaubt, sondern für die Stabilisierung geradezu notwendig sind, wurde ebenfalls experimentell bestätigt [5]. Demnach ist asymmetrisches MDFC symmetrischem MDFC und auch (E)TDAS überlegen. Dabei ist eine asymmetrische Wahl der Vorfaktoren $k_{m a}, k_{m b}$ nicht nur unter dem Aspekt einer optimalen Anpassung der Übertragungsfunktion der Regelung an das dynamische System zu sehen, sondern kann auch dazu verwendet werden, die schwer zu kontrollierenden Sattelpunkte zu stabilisieren. Das ist mit den anderen bekannten Zeitverzögerungsmethoden (E)TDAS nicht möglich, da zu diesem Zweck ein Symmetriebruch vonnöten ist, der ein Verschwinden des Regelsignals an der Stabilitätsgrenze verhindert und stattdessen eine geeignete Ankopplung an das zu kontrollierende System ermöglicht. Neben MDFC ist auch NFF in der Lage, instabile Sattelpunkte zu kontrollieren, was beide Methoden im Hinblick auf Anwendbarkeit, Effizienz und Erfolg den anderen bekannten Regelungsmethoden überlegen macht.

Das volle Potential von NFF und MDFC in Bezug auf die Kontrolle raum-zeitlich chaotischer Dynamik muss noch weiter getestet werden. So lag neben der Fixpunktstabilisierung ein Fokus auf der Umwandlung chaotischer Dynamik in laufende ebene Wellen, was für das Fitzhugh-Nagumo-System (einem Prototypen für die menschliche Herzdynamik) sowohl mit NFF als auch mit MDFC möglich ist (siehe Anhang). Auf diese Art und Weise sind beide Regelungen vielleicht eine zukünftige schonende Alternative zu der bisher angewendeten Defibrilation bei Herz-Rhythmusstörungen. Um die Aussagekraft dieser Ergebnisse einordnen zu können, wurde zudem die zweidimensionale Ginzburg-Landau-Gleichung unter dem gleichen Gesichtspunkt untersucht. Dabei hat sich herausgestellt, dass aufgrund der bekannten Torsionsbeschränkungen im Gegensatz zu (E)TDAS und P-Regelung mit MDFC eine erfolgreiche Stabilisierung von ebenen Wellen vorgenommen werden kann. Relevanz erhalten diese Ergebnisse aufgrund der Tatsache, dass die komplexe GinzburgLandau-Gleichung Prototyp für eine ganze Reihe räumlich ausgedehnter Systeme ist. Daneben zeigen die anderen diesbezüglich präsentierten Beispiele, dass MDFC nicht nur neue 
Möglichkeiten im Hinblick auf die Unterdrückung chaotischer Dynamik, sondern für zukünftige Anwendungen eine große Palette an ausgefeilten Manipulationen dieser Dynamik offeriert. So sind komplexe raum-zeitliche Strukturen wie sektorgerichtete oder gescherte ebene Wellen, interagierende Spiralwellen oder auch Spiralfallen möglich. Für die zuletzt genannte Dynamik kommt es in den Bereichen, in denen MDFC lokal angewendet wird (Kontrollzellen) zu identischer Synchronisation. In weiteren Untersuchungen hat sich herausgestellt, dass unter dem Gesichtspunkt der Synchronisation eine lokale bidirektionale zeitverzögerte bzw. nicht zeitverzögerte Rückkopplung einer unidirektionalen im Hinblick auf die Anwendbarkeit überlegen ist. Die Überprüfung des verwendeten frequenzverdoppelten Lasers bezüglich Synchronisation bei externer sinusförmiger Modulation ergab daraufhin die Existenz ausgedehnter Synchronisationsgebiete, an deren Rändern chaotische Phasensynchronisation vorliegt, die im Zuge dieser Arbeit experimentell für ein solches Lasersystem nachgewiesen werden konnte.

\subsection{Ausblick}

Bezüglich der Regelung von räumlich ausgedehnten Systemen mit NFF und MDFC wurde erst der Anfang gemacht, so dass noch Spielraum für weitere Analysen und Kopplungsschemata bleibt. Wünschenswert wäre zudem eine Überprüfung dieser theoretischen Erkenntnisse an einem geeigneten Experiment. In Anbetracht des hochdimensionalen Parameterraumes für MDFC und NFF ist darüber hinaus noch Klärungsbedarf für die optimale (automatisierte) Parametereinstellung in einem Experiment (mit Instationarität). Hier ist eine Nachführung der Regelparameter in u. U. weiten Bereichen erforderlich. Anfänge wurden bereits durch die in Kap. 5 beschriebene Regelung der Verzögerungszeiten für MDFC bzw. der Filterresonanzfrequenzen für NFF gemacht. In Zukunft kann diese Einstellung und Nachführung der Parameter vielleicht durch einen intelligenten Lernalgorithmus vorgenommen werden, der eventuell im Hinblick auf räumlich ausgedehnte Systeme zudem die Anzahl der benötigten Kontrollzellen weiter reduzieren kann, indem die Orte für den Kontrolleingriff je nach gewünschter Zieldynamik optimal gewählt werden. Durch diese weitere Effizienzsteigerung stünden dann mit MDFC und NFF zwei universelle Regelungen zur Verfügung, die in weiten Bereichen anwendbar sind. 


\section{Anhang A}

\section{Chaos-Kontrollmethoden ohne Zeitverzögerung}

\section{A.1 OGY-Kontrolle}

Ein instabiler periodischer Orbit oder Fixpunkt einer iterierten Abbildung soll durch die Anwendung kleiner und sorgfältig berechneter Störungen eines zugänglichen Systemparameters stabilisiert werden. Die schematische Darstellung dieses Sachverhaltes zeigt die Abb. A.1, in der sich die Dynamik zunächst parallel zu der stabilen Richtung dem Fixpunkt nähert. Je geringer aber der Abstand zum instabilen Fixpunkt wird, desto mehr dominiert die Dynamik der instabilen Richtung, wenn die Annäherung nicht exakt entlang der stabilen Mannigfaltigkeit vonstatten geht. Die Dynamik entfernt sich daraufhin wieder aus der Nähe des Fixpunktes, es sei denn, die Lage des Fixpunktes wird durch kleine Störungen so verschoben, dass bei der nächsten Iteration die Dynamik genau auf die stabile Mannigfaltigkeit des instabilen Fixpunktes fällt. In diesem Falle würde sich die Dynamik dann selbständig auf den Fixpunkt zubewegen.

Um die für diese OGY-Kontrolle (Ott, Grebogi, Yorke, siehe Abschnitt 2.2) [106] nötigen Parameter zu ermitteln, wird die $d$-dimensionale Abbildung

$$
\mathbf{x}_{n+1}=\mathbf{f}\left(\mathbf{x}_{n}, p\right)
$$

mit dem $n$-fach iterierten Systemzustand $\mathbf{x}_{n}$ betrachtet. Für kontinuierliche Systeme steht für diesen Zweck eine Poincaré-Ebene des Systems zur Verfügung, wobei $p$ einen zugänglichen Systemparameter darstellt, der entsprechend variiert werden kann. Dabei verändert sich die Position des betrachteten instabilen Fixpunktes $\mathbf{x}_{0}\left(p_{0}\right)$ glatt mit $p$, bis der Wert $p_{0}$ bei Erreichen der Zieldynamik angenommen wird. Bei der Anwendung kleiner Störungen kann dann in einer kleinen Umgebung des Fixpunktes $\mathbf{x}_{0}$ und bei kleinen Kontrollkräften $\left|p_{n}-p_{0}\right| \ll 1$ durch

$$
\mathbf{x}_{0}(p) \approx \mathbf{x}_{0}\left(p_{0}\right)+\mathbf{g} \Delta p
$$


(a)

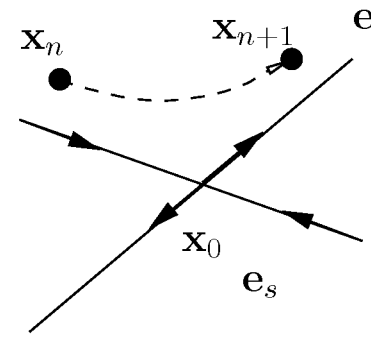

(b)

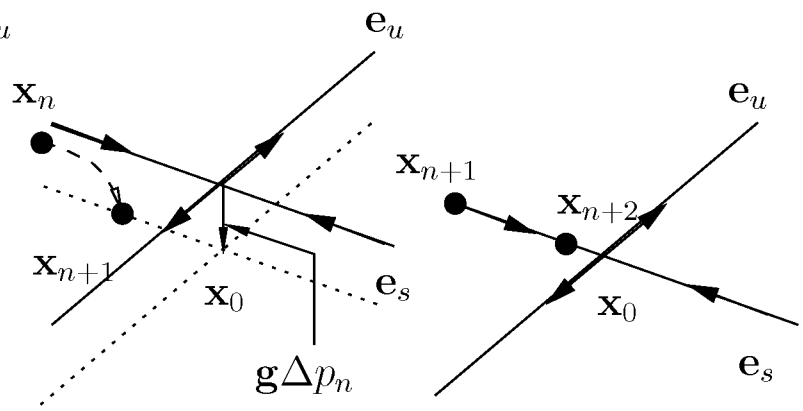

Abb. A.1: Schematische Darstellung der OGY-Regelung. (a) Annäherung an den Fixpunkt $\mathbf{x}_{0}$ parallel der stabilen Richtung $\mathbf{e}_{s}$, wobei der Einfluss der instabilen Richtung $\mathbf{e}_{u}$ letztendlich wiederum zu einer erneuten Entfernung der Dynamik vom Fixpunkt führt. (b) Verschiebung des Fixpunktes durch den Fixpunktvektor g so, dass sich die Systemdynamik dem Fixpunkt während der nächsten Iteration $\mathbf{x}_{n+1}$ entlang der stabilen Mannigfaltigkeit nähert (c).

mit dem Verschiebungsvektor

$$
\mathbf{g}=\left.\frac{\partial \mathbf{x}_{0}(p)}{\partial p}\right|_{p=p_{0}} \approx \frac{\mathbf{x}_{0}(p)-\mathbf{x}_{0}\left(p_{0}\right)}{\Delta p}
$$

approximiert werden. Hierbei ist $\mathrm{zu}$ beachten, dass $\mathrm{g}$ bestimmt werden muss, bevor das Kontrollgesetz zur Stabilisierung von $\mathbf{x}_{0}$ berechnet werden kann. Linearisierung des dynamischen Systems (A.1) am Fixpunkt $\mathbf{x}_{0}\left(p_{0}\right)$ führt wiederum unter Verwendung der Jacobi-Matrix

$$
D \mathbf{f}\left(\mathbf{x}_{0}(p)\right)=\left.\frac{\partial \mathbf{f}}{\partial \mathbf{x}}\right|_{\mathbf{x}_{0}(p)} \approx D \mathbf{f}\left(\mathbf{x}_{0}\left(p_{0}\right)\right)+\left.\frac{\partial \mathbf{f}}{\partial p}\right|_{p=p_{0}} \Delta p
$$

zu der Gleichung

$$
\begin{aligned}
\mathbf{x}_{n+1}-\mathbf{x}_{0}(p) & \approx \mathbf{x}_{n+1}-\left(\mathbf{x}_{0}\left(p_{0}\right)+\mathbf{g} \Delta p\right) \\
& \approx D \mathbf{f}\left(\mathbf{x}_{0}\left(p_{0}\right)\right)\left(\mathbf{x}_{n}-\mathbf{x}_{0}\left(p_{0}\right)-\mathbf{g} \Delta p\right),
\end{aligned}
$$

was durch das Einsetzen von Gl. (A.2) zu

$$
\mathbf{x}_{n+1}-\mathbf{x}_{0}\left(p_{0}\right)=\mathbf{g} \Delta p+D \mathbf{f}\left(\mathbf{x}_{0}\left(p_{0}\right)\right)\left(\mathbf{x}_{n}-\mathbf{x}_{0}\left(p_{0}\right)-\mathbf{g} \Delta p\right)
$$

führt. Die Elemente von $D \mathbf{f}\left(\mathbf{x}_{0}\left(p_{0}\right)\right)$ des ungestörten Systems können bei nicht bekannten Systemgleichungen aus einer gemessenen Zeitreihe, anschließender geeigneter Einbettung und weiterer Analyse der Dynamik in der Nähe des betrachteten instabilen Fixpunktes 
berechnet werden. Dabei seien die Eigenwerte von $D \mathbf{f}$ durch $\lambda_{u}$ und $\lambda_{s}$ für die jeweils instabile und stabile Richtung des Fixpunktes sowie die zugehörigen Eigenvektoren durch $\mathbf{e}_{u}$ und $\mathbf{e}_{s}$ bezeichnet. Durch die Einführung der kontravarianten Vektoren $\mathbf{v}_{u}$ und $\mathbf{v}_{s}$ anhand von

$$
\mathbf{v}_{s} \cdot \mathbf{e}_{s}=\mathbf{v}_{u} \cdot \mathbf{e}_{u}=1 \quad \mathbf{v}_{s} \cdot \mathbf{e}_{u}=\mathbf{v}_{u} \cdot \mathbf{e}_{s}=0
$$

kann die Jacobi-Matrix des frei laufenden Systems in

$$
D \mathbf{f}\left(\mathbf{x}_{0}\left(p_{0}\right)\right)=\lambda_{u} \mathbf{e}_{u} \cdot \mathbf{v}_{u}+\lambda_{s} \mathbf{e}_{s} \cdot \mathbf{v}_{s}
$$

umgeschrieben werden. Zudem lässt sich die Annäherung der Dynamik an den Fixpunkt entlang der stabilen Mannigfaltigkeit unter Verwendung der kontravarianten Vektoren in der Form

$$
\mathbf{v}_{u} \cdot\left(\mathbf{x}_{n+1}-\mathbf{x}_{0}\left(p_{0}\right)\right)=0
$$

ausdrücken. Wenn $\mathbf{x}_{n+1}$ auf die stabile Richtung von $\mathbf{x}_{0}\left(p_{0}\right)$ gefallen ist, kann das Kontrollsignal auf Null gesetzt werden und die Trajektorie wird den Fixpunkt mit der geometrischen Rate $\lambda_{s}$ selbständig erreichen. Solange also $\mathbf{x}_{n}-\mathbf{x}_{0}\left(p_{0}\right)$ klein genug ist, können die Gleichungen (A.9) und (A.8) in Gl. (A.6) eingesetzt werden. Mit $\Delta p=c_{n}$ und

$$
\mathbf{x}_{n+1}-\mathbf{x}_{0}\left(p_{0}\right) \approx \mathbf{g} \Delta p+\left(\lambda_{u} \mathbf{e}_{u} \cdot \mathbf{v}_{u}+\lambda_{s} \mathbf{e}_{s} \cdot \mathbf{v}_{s}\right)\left(\mathbf{x}_{n}-\mathbf{x}_{0}\left(p_{0}\right)-\mathbf{g} \Delta p\right),
$$

ergibt sich als notwendige Bedingung für eine erfolgreiche Fixpunktstabilisierung der Ausdruck

$$
\mathbf{v}_{u} \cdot\left(\mathbf{x}_{n+1}-\mathbf{x}_{0}\left(p_{0}\right)\right) \approx \mathbf{v}_{u} \cdot \mathbf{g} \underbrace{\Delta p}_{c_{n}}+\lambda_{u} \underbrace{\mathbf{e}_{u} \cdot \mathbf{v}_{u}^{2}}_{\mathbf{v}_{u}}(\mathbf{x}_{n}-\mathbf{x}_{0}\left(p_{0}\right)-\mathbf{g} \underbrace{\Delta p}_{C_{n}}) \stackrel{!}{=} 0 .
$$

Dieses Resultat führt wiederum zu

$$
c_{n}=C\left(\mathbf{x}_{n}-\mathbf{x}_{0}\left(p_{0}\right)\right)
$$

mit

$$
C=\frac{\lambda_{u} \mathbf{v}_{u}}{\left(\lambda_{u}-1\right) \mathbf{v}_{u} \cdot \mathbf{g}}
$$

Für die Berechnung dieser Gleichung wurde stillschweigend angenommen, dass $\mathbf{g} \cdot \mathbf{f}_{u} \neq$ 0 und damit auch die Abweichung vom Sollzustand $c_{n} \propto \mathbf{x}_{n}-\mathbf{x}_{0}\left(p_{0}\right)$ klein ist. Diese Annahmen treffen aber nur für eine lokale kleine Umgebung von $\mathbf{x}_{0}\left(p_{0}\right)$ zu. Zum Vermeiden einer zu starken Veränderung des dynamischen Systems wird $\Delta p$ global auf Null gesetzt, wenn $\left|c_{n}\right|$ zu groß wird. Man erhält somit für die Regelung zu einem fest vorgegebenen Schwellwert $\delta$ die Kontrollkraft

$$
\Delta p= \begin{cases}c_{n} & : \text { wenn }\left|c_{\mathrm{n}}\right|<\delta \\ 0 & : \text { sonst. }\end{cases}
$$


Damit kann die eigentliche Kontrollprozedur nur dann angewendet werden, wenn die Trajektorie in der Nachbarschaft der gewünschten Zieldynamik ist, was unter Umständen bei kleiner $\epsilon$-Umgebung um den Fixpunkt entsprechend lange dauern kann. Um diese Zeitspanne zu verkürzen, kann die Dynamik durch kleine gezielte Störungen eventuell schneller in die gewünschte Nachbarschaft des Fixpunktes gebracht werden (Targetting). Zudem gibt es noch weitere Nachteile von OGY, die eine experimentelle Implementierung erschweren. Da durch die verwendete Poincaré-Abbildung die Veränderung der Parameter immer zeitdiskret ist, können z. B. nur Fixpunkte stabilisiert werden, deren maximaler Lyapunov-Exponent klein im Vergleich zum reziproken Zeitintervall zwischen zwei Parameteränderungen ist. Pro Periode kann nur ein einziger Kontrolleingriff vorgenommen werden, was aber unter Umständen bei sehr hoher Instabilität nicht ausreichend sein kann. Ebenso ist aufgrund des in der Praxis immer auftretenden Messrauschens ständig eine Korrektur des Regelsignals erforderlich, d.h. der Systemzustand muss fortlaufend mit dem Computer überwacht werden, um eine aktuelle Berechnung des benötigten Kontrollsignals zu erhalten. Um diese Einschränkungen zu umgehen, muß das beschriebene OGY-Verfahren verändert werden [41].

Führt man sich die OGY-Kontrolle einmal genauer vor Augen, so handelt es sich in erster Linie dabei um eine lokal angewendete Variante des üblichen Pole-Placement (siehe Abschnitt 2.1), das schon Gegenstand vielfältiger Untersuchungen vor der Anwendung auf nichtlineare Systeme war.

\section{A.2 OPF-Kontrolle}

OPF (occasional proportional feedback) kann als eine eindimensionale Version der OGYRegelung angesehen werden. Ein Vorteil von OPF gegenüber OGY liegt allerdings darin, dass für eine erfolgreiche Regelung keine vollständige Zeitreihenanalyse betrieben werden muss, um z. B. eine Stabilisierung von periodischen Orbits herbeizuführen. Ein Hauptelement der Regelung ist ein Fensterkomparator, der eine Aktivierung der Regelgröße erst ermöglicht, wenn die skalare, zu bestimmten Zeiten $t_{n}=t_{0}+n T, n \in \mathbb{N}$ gesampelte Eingangsgröße $y\left(t_{n}\right)=\mathbf{g}\left(\mathbf{x}\left(t_{n}\right)\right)$ in einem vorher eingestellten Bereich $2 \Delta y_{\max }$ um eine gewählte Referenzamplitude $y^{0}$ liegt. Dieses Referenzsignal kann z. B. durch eine Tiefpassfilterung generiert werden. $T$ ist in der Größenordnung der Relaxationszeit des Systems, d.h. für die Störung

$$
u\left(t_{n}\right)= \begin{cases}k\left(y\left(t_{n}\right)-y^{0}\right) & : \text { wenn } t \in\left[t_{n}, t_{n}+\theta\right] \text { und }|u|<u_{\max } \\ 0 & : \text { sonst }\end{cases}
$$

mit der Applikationszeit $\theta$, die deutlich kürzer als die Periode des zu stabilisierenden periodischen Orbits ist. $u_{\max }$ ist der vorher festgelegte Schwellwert für das Fenster um den zu stabilisierenden Amplitudenwert. Dieser muss natürlich genau wie die Relaxationszeit 


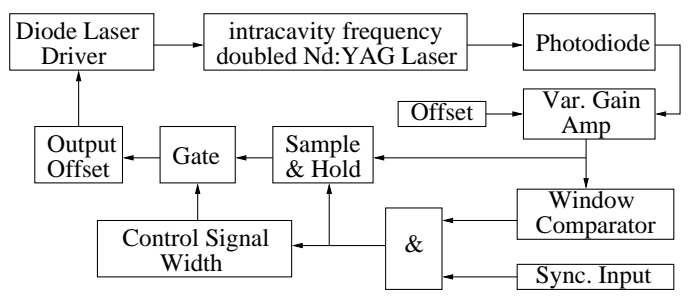

Abb. A.2: OPF-Regelung zur Stabilisie-
rung periodischer Orbits eines frequenz-
verdoppelten Lasers mit Typ-I Chaos.
Das Regelsignal wird nur aktiviert, wenn
der gesampelte Messwert zu einer be-
stimmten Zeit in ein vorher eingestelltes
(Amplitudenwert-)Fenster fällt.

des Systems vorher schon einigermaßen bekannt sein, so dass dann zusätzlich mit Hilfe einer Variation von $k$ im besten Fall eine Stabilisierung erzielt werden kann. Mit dieser Regelung konnten erfolgreich periodische Orbits bei einem frequenzverdoppelten Nd:YAGLaser mit Typ-I-Chaos (alle Moden sind parallel zueinander polarisiert) stabilisiert werden [63]. Der schematische Aufbau hierfür ist Abb. A.2 zu entnehmen. 


\section{Anhang B}

\section{Kontrollierbarkeitskriterium im allgemeinsten Fall}

Die Behandlung des allgemeinsten Falls einer Rückkopplung zur Stabilisierung eines Sattelpunktes verläuft analog zu der des in Kapitel 4 betrachteten Spezialfalls. Ausgangspunkt ist die Annahme einer zeitverzögerten Regelung $\mathbf{u}=\left(u_{1}, u_{2}, \ldots, u_{n}\right)^{t r}$, die an alle Gleichungen des $n$-dimensionalen dynamischen Systems koppelt und auch Linearkombinationen aller durch die Differentialgleichungen beschriebenen Variablen $x_{1}, \ldots, x_{n}$ als Eingangssignale für die einzelnen Rückkopplungen $u_{i}$ verwendet. Unter diesem Gesichtspunkt berechnet sich die allgemeinste Form der kontinuierlichen charakteristischen Funktion $S(\lambda)$ (mit den Laplace-Transformierten $\partial \bar{u}_{i} / \partial \bar{x}_{j}$ der einzelnen Regelungskomponenten bezüglich der jeweils verwendeten Variablen) zu

$$
\begin{gathered}
S(\lambda)=\operatorname{det}[\Delta(\lambda)]=\operatorname{det}\left[\lambda I-D \mathbf{f}\left(\mathbf{x}_{0}\right)-U\left(\mathbf{x}_{0}, \lambda\right)\right] \\
=\lambda^{n}+\sum_{j=0}^{n-1} c_{j} \lambda^{j}+\sum_{\substack{i_{1}=0 \\
j_{1}=0}}^{n-1} \frac{\partial \bar{u}_{i_{1}}}{\partial \bar{x}_{j_{1}}} \sum_{l=0}^{n-1} d_{i_{1} j_{1} l} \lambda^{l}+\frac{1}{2} \sum_{\substack{i_{1}, i_{2}=0 \\
j_{1}, j_{2}=0}}^{n-1} \frac{\partial \bar{u}_{i_{1}}}{\partial \bar{x}_{j_{1}}} \frac{\partial \bar{u}_{i_{2}}}{\partial \bar{x}_{j_{2}}} \sum_{l=0}^{n-1} e_{i_{1} i_{2} j_{1} j_{2} l} \lambda^{l} \\
+\ldots+\frac{1}{n} \sum_{\substack{i_{1}, \ldots, i_{n}=0 \\
j_{1}, \ldots, j_{n}=0}}^{n-1} \frac{\partial \bar{u}_{i_{1}}}{\partial \bar{x}_{j_{1}}} \frac{\partial \bar{u}_{i_{2}}}{\partial \bar{x}_{j_{2}}} \cdots \frac{\partial \bar{u}_{i_{n}}}{\partial \bar{x}_{j_{n}}} \sum_{l=0}^{n-1} r_{i_{1} \ldots i_{n} j_{1} \ldots j_{n} l} \lambda^{l}
\end{gathered}
$$

bestehend aus einem rein polynomialen Anteil mit den Parametern $c_{j}$ (stammt von dem freilaufenden dynamischen System) und mehreren gemischten polynomial-transzendenten Anteilen (resultieren aus der Ankopplung des Regelsignals $\mathbf{u}$ an das dynamische System und der Wechselwirkung der verschiedenen Regelungskomponenten bei der Berechnung der charakteristischen Gleichung (B.2)). Diese Parameter sind alle endlich, so dass sich zusammen mit einer beschränkten, stabilen Regelung der Grenzfall $\lim _{\lambda \rightarrow \infty} S(\lambda) \rightarrow \infty$ 
ergibt. Wird die charakteristische Gleichung (B.2) für $\lambda=0$ betrachtet, so folgt

$$
\begin{aligned}
S(0)=c_{0}+\sum_{\substack{i_{1}=0 \\
j_{1}=0}}^{n-1} \frac{\partial \bar{u}_{i_{1}}}{\partial \bar{x}_{j_{1}}} d_{i_{1} j_{1} 0}+\frac{1}{2} \sum_{\substack{i_{1}, i_{2}=0 \\
j_{1}, j_{2}=0}}^{n-1} \frac{\partial \bar{u}_{i_{1}}}{\partial \bar{x}_{j_{1}}} \frac{\partial \bar{u}_{i_{2}}}{\partial \bar{x}_{j_{2}}} e_{i_{1} i_{2} j_{1} j_{2} 0} \\
\\
\quad+\ldots+\frac{1}{n} \sum_{\substack{i_{1}, \ldots, i_{n}=0 \\
j_{1}, \ldots, j_{n}=0}}^{n-1} \frac{\partial \bar{u}_{i_{1}}}{\partial \bar{x}_{j_{1}}} \frac{\partial \bar{u}_{i_{2}}}{\partial \bar{x}_{j_{2}}} \cdots \frac{\partial \bar{u}_{i_{n}}}{\partial \bar{x}_{j_{n}}} r_{i_{1} \ldots i_{n} j_{1} \ldots j_{n} 0} .
\end{aligned}
$$

Sind dabei alle Rückkopplungsglieder

$$
\begin{aligned}
u_{i}=k_{i_{1}}\left[\left(1-R_{i_{1}}\right) \sum_{r=1}^{\infty} R_{i_{1}}^{r-1} x_{i_{1}}(t-\right. & \left.\left.r \tau_{i_{1}}\right)-x(t)\right]+\ldots \\
& +k_{i_{n}}\left[\left(1-R_{i_{n}}\right) \sum_{r=1}^{\infty} R_{i_{n}}^{r-1} x_{i_{n}}\left(t-r \tau_{i_{n}}\right)-x(t)\right]
\end{aligned}
$$

vom (E)TDAS-Typ ${ }^{1}$, so verschwindet analog zu Kapitel 4 der Einfluss aller Regelkomponenten bei $\lambda=0$. Im Detail führt das zu

$$
S(0)=\operatorname{det}\left[-D \mathbf{f}\left(\mathbf{x}_{0}\right)\right]=(-1)^{n} \operatorname{det}\left[D \mathbf{f}\left(\mathbf{x}_{0}\right)\right]=\prod_{j=1}^{n}\left(-e_{j}\right)=c_{0}
$$

mit den Eigenwerten $e_{j}$ der Jacobi-Matrix $D \mathbf{f}\left(\mathbf{x}_{0}\right)$ des instabilen ungeregelten Systems. Bei Betrachtung eines Sattelpunktes (ungerade Anzahl reeller, positiver Eigenwerte) ist $S(0)<0$, was zusammen mit dem Grenzfall $\lim _{\lambda \rightarrow \infty} S(\lambda) \rightarrow \infty$ die Existenz mindestens eines mit (E)TDAS nicht stabilisierbaren Eigenwertes impliziert.

Ein betrachteter Fixpunkt ist hingegen stabil, wenn die Kontrollparameter so gewählt werden können, dass die Funktion $S(\lambda)$ keine Nullstellen in der rechten Hälfte der komplexen Ebene besitzt (d.h. keine Nullstellen mit positivem Realteil). Wird anstelle von (E)TDAS aus Gl. (B.4) MDFC gemäß

$$
u_{i}=k_{0}+\sum_{m=1}^{M} k_{m a i_{1}} x_{i_{1}}\left(t-\tau_{m i_{1}}\right)-k_{m b i_{1}} x_{i_{1}}(t)+\ldots+k_{m a i_{n}} x_{i_{n}}\left(t-\tau_{m i_{n}}\right)-k_{m b i_{n}} x_{i_{n}}(t)
$$

angewendet, so verändert sich die Laplace-Transformierte aus Gl. (B.4) zu

$$
\frac{\bar{u}_{i_{p}}}{\bar{x}_{i_{q}}}(\lambda)=\sum_{m=1}^{M} k_{m a i_{q}} e^{-\lambda \tau_{m i_{q}}}-k_{m b i_{q}}, \quad q=1, \ldots, n,
$$

\footnotetext{
${ }^{1} \mathrm{Im}$ eigentlichen Sinne müsste in diesem Fall $\tau_{i_{1}}=\ldots=\tau_{i_{n}}=\tau$ gelten, da (E)TDAS laut Definition [141] nur eine einzige Verzögerungszeit $\tau$ im gesamten Regelkreis verwendet.
} 
wodurch Gl. (B.7) im Gegensatz zu Gl. (B.4) nicht mehr für $\lambda=0$ verschwindet, sondern von den Differenzen der einzelnen Kontrollparameter $k_{m a i_{q}}-k_{m b i_{q}}, q=1, \ldots, n$ abhängt. Auf diese Weise kann die charakteristische Funktion $S(0)$ aus Gl. (B.3) mit

$$
\frac{\bar{u}_{i_{p}}}{\bar{x}_{i_{q}}}(0)=\sum_{m=1}^{M} k_{m a i_{q}}-k_{m b i_{q}}, \quad l=1, \ldots, n,
$$

an der Stelle $\lambda=0 \mathrm{zu}$ positiven Werten verschoben werden. Daraus lässt sich zusammen mit dem Grenzfall $\lim _{\lambda \rightarrow \infty} S(\lambda) \rightarrow \infty$ folgern, dass eine gerade Anzahl an Nullstellen für $S(\lambda)$ in der rechten Hälfte der komplexen Ebene vorliegt, deren genaue Anzahl sowohl von dem betrachteten dynamischen System als auch von den Kontrollparametern abhängt. So ist durch die Verschiebung von $S(0)$ zu positiven Werten u. U. schon eine erfolgreiche Stabilisierung vorgenommen worden. Sollte das noch nicht der Fall sein, so besteht nun kein fundamentales Hindernis mehr für die Stabilisierung des betrachteten Sattelpunktes durch einen zusätzlichen Kontroller des (E)TDAS-Typs (2.11).

Daran ist wieder die Notwendigkeit der Anwendung von mindestens zwei verschiedenen Verzögerungszeiten für die Stabilisierung von Sattelpunkten zu erkennen. Der erste verzögerte Term mit asymmetrischen (unterschiedlichen) Verstärkungsfaktoren verändert das dynamische System dahingehend, dass $S(0)>0$ gewährleistet ist. Der zweite Zeitverzögerungsterm aus Gl. (B.6) stabilisiert schließlich dieses neue, veränderte System. Durch die Verwendung der asymmetrischen Vorfaktoren $k_{m a i_{q}}, k_{m b i_{q}}$ handelt es sich allerdings bei Gl. (B.6) um eine invasive Regelung. Dieser Sachverhalt kann durch geeignete Anpassung des Parameters $k_{0}$ aus Gl. (B.6), der nicht in die Stabilitätsanalyse und damit auch nicht in die charakteristische Gleichung (B.2) eingeht, beseitigt werden, wodurch schließlich eine nichtinvasive Regelung gewährleistet ist. 


\section{Anhang C}

\section{Räumlich ausgedehnte Systeme}

\section{C.1 Ginzburg-Landau-Gleichung (Stabilitätsanalyse)}

Um die Stabilität von ebenen Wellen $f=f(\mathbf{x}, t)$ mit Wellenvektor $\mathbf{k}$ für die GLE (6.5) in Gegenwart einer homogenen Regelung $u(t)$ zu testen, wird eine Störung der Form $f g=f+B$ angenommen, was nach der Entwicklung von $B$ in Fourier-Moden zu

$$
B=\sum_{\mathbf{l}} \mu_{\mathbf{l}} e^{i \mathbf{l} \cdot \mathbf{x}}
$$

führt. Weiteres Einsetzen in Gl. (6.5) und anschließende Linearisierung resultiert zusammen mit der (laplacetransformierten) Regelung $u_{R}$ in der Matrix

$$
A=-\left(\begin{array}{cc}
\alpha l^{2}+\beta f_{0}^{2}+2 \alpha \mathbf{k} \cdot \mathbf{l} & \beta f_{0}^{2} \\
\beta^{*} f_{0}^{2} & \alpha^{*} l^{2}+\beta^{*} f_{0}^{2}-2 \alpha^{*} \mathbf{k} \cdot \mathbf{l}
\end{array}\right)+\left(\begin{array}{cc}
u_{R} & 0 \\
0 & u_{R}^{*}
\end{array}\right)
$$

mit $\alpha=1+i a, \beta=1+i b$ und $\|\mathbf{l}\|=l$. Über die Stabilität der ebenen Wellen entscheidet der Eigenwert mit dem größten Realteil, der die charakteristische Gleichung $\operatorname{det}(\lambda I-A)$ mit

$$
(z(\lambda)-\lambda-2 \alpha \mathbf{k} \cdot \mathbf{l})\left(z^{*}(\lambda)-\lambda+2 \alpha^{*} \mathbf{k} \cdot \mathbf{l}\right)-\left(1+b^{2}\right) f_{0}^{4}=0
$$

erfüllt, wobei $z(\lambda)=u_{R}(\lambda)-\alpha l^{2}-\left(1+i b f_{0}^{2}\right)$ und $I$ die Einheitsmatrix bezeichnet. Ist dieser Realteil $<0$, so sind die ebenen Wellen stabil. Ob diese Wellen auch Lösungen der freilaufenden GLE (6.5) sind oder von der Regelung generiert werden, hängt wiederum von den Rückkopplungsparametern ab (vgl. Kapitel 6).

\section{C.2 Ginzburg-Landau-Gleichung (Fixpunkt)}

Ein Beispiel für die Stabilisierung des instabilen Fixpunktes $f(\mathbf{x})=0$ für die zweidimensionale Ginzburg-Landau-Gleichung (6.5) ist in Abb.C.1 für die Parameterwerte $a=2$ 
und $b=-1.2 \mathrm{zu}$ sehen, die für das freilaufende System $(u=0)$ zu turbulenter Dynamik führen (Abb. C.1a). Gemäß den Ausführungen aus Abschnitt 4.2 und Abschnitt 6.3 erwies sich (E)TDAS bei dieser Parameterkombination als nicht geeignet, um eine erfolgreiche Fixpunktstabilisierung herbeizuführen. Von daher wird MDFC mit drei verschiedenen Verzögerungszeiten an ausgewählten lokalen Kontrollzellen angewendet, um diese chaotischen Amplitudenfluktuationen zu unterdrücken. Im Detail lautet das an der Kontrollzelle $\mathcal{C}_{j}$ gemessene und auch dort wieder angewendete Regelsignal

$$
u_{j}(t)=\sum_{m=1}^{3} k_{m a} f\left(\mathcal{C}_{j}, t-\tau_{m}\right)-k_{m b} f\left(\mathcal{C}_{j}, t\right),
$$

wobei die Verzögerungszeiten auf die Werte $\tau_{1}=27, \tau_{2}=69, \tau_{3}=123$ und die Verstärkungsfaktoren auf $k_{1 a}=0.2, k_{2 a}=0.25, k_{3 a}=0.3, k_{1 b}=0.9, k_{2 b}=0.9, k_{3 b}=0$ fixiert sind. Das verwendete Kopplungsschema kann zudem der Abb. 6.7 entnommen werden. Abb. C.1b zeigt die Transienten der Dynamik, kurz nachdem die Regelung (C.4) einge-
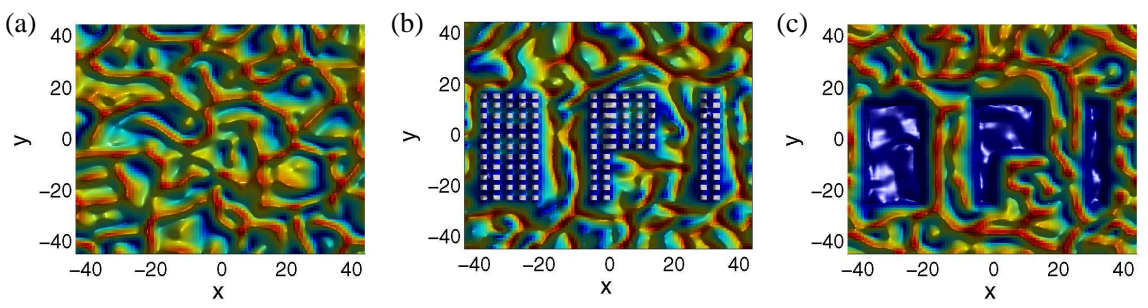

\begin{abstract}
Abb. C.1: Farbkodierte Darstellung der raum-zeitlichen Entwicklung der Amplitude der Lösungsfunktion $f$ für die zweidimensionale Ginzburg-Landau-Gleichung (6.5) mit den Parameterwerten $a=2$ und $b=-1.2$. (a) Freilaufendes System $(\mathbf{u}=0)$, (b) Transienten der kontrollierten Dynamik, wobei MDFC entsprechend Gl. (C.4) an den weiß markierten Kontrollzellen angewendet wird. (c) Lokale Unterdrückung der chaotischen Amplitudenfluktuationen innerhalb der Kontrollregion, was sich in nur noch sehr kleinen vereinzelten Amplitudenwerten äußert.
\end{abstract}

schaltet wurde. Die Kontrollzellen sind in dieser Darstellung exemplarisch weiß markiert und in Form der Buchstaben DPI angeordnet. Das dort eingespeiste MDFC-Signal mit asymmetrischen Vorfaktoren führt im Gegensatz zu (E)TDAS innerhalb des Regelgebietes zu der Reduzierung der Dynamik auf den betrachteten Fixpunkt (Abb. C.1c), wobei allerdings aus Übersichtlichkeitsgründen die Kontrollzellen in dieser Darstellung nicht mehr kenntlich gemacht wurden. Ähnliche Eigenschaften von MDFC für gekoppelte iterierte Abbildungen wurden kürzlich in [91,92] veröffentlicht. 


\section{C.3 Fitzhugh-Nagumo-System}

Im Hinblick auf die störende Spiraldynamik im menschlichen Herzgewebe (Herzflimmern) wird anstelle der komplexen Ginzburg-Landau-Gleichung (6.5) oft das Fitzhugh-NagumoSystem

$$
\begin{aligned}
& \frac{\partial u}{\partial t}=D_{u} \nabla^{2} u+\epsilon^{-1} u(1-u)\left(u-\frac{v+b}{a}\right) \\
& \frac{\partial v}{\partial t}=D_{v} \nabla^{2} v+u-v
\end{aligned}
$$

mit periodischen Randbedingungen untersucht. Die System-Parameter sind auf $a=0.75$, $b=0.06, \epsilon=1 / 12, D_{u}=1$ und $D_{v}=0$ fixiert. Zusätzlich wird jeweils auf der gesamten Breite alle 70 (Zeiteinheiten) eine Erregung an der Position $y=77$ für MDFC (Abb. C.2a-c) bzw. $y=49$ für NFF (Abb. C.2d-f) vorgenommen. Für die Integration von Gl. (C.5) wird das in $90 \times 90$ Zellen unterteilte dynamische Gebiet entsprechend des verwendeten Barkley-Codes [17,18] zeitlich mit einem Euler-Verfahren und räumlich mit einer Neun-Punkt-Approximation gelöst. Beeinflusst wird die auftretende Spiraldynamik mittels ausgewählter Kontrollzellen $\mathcal{C}_{j}$, die in Abb. C.2 weiss markiert sind und über die eine geeignete Regelung auf das System einwirkt.

Zunächst sei hierfür MDFC mit den Parametern $\tau_{1}=10.2, \tau_{2}=45, \tau_{3}=78.2$ sowie $k_{1 a}=$ $0.13, k_{1 b}=0.31, k_{2 a}=0.41, k_{2 b}=0.34, k_{3 a}=0.16, k_{3 b}=0$ unter Berücksichtigung des Kopplungsschemas aus Abb. 6.7g angewendet. Im Detail lautet das an der Kontrollzelle $\mathcal{C}_{j}$ angewendete Regelsignal

$$
\begin{aligned}
p_{j}= & k_{1 a} u_{j}\left(t-\tau_{1}\right)+k_{2 a} u_{j}\left(t-\tau_{2}\right)+k_{3 a} u_{j}\left(t-\tau_{3}\right) \\
& -\left(k_{1 b}+k_{2 b}+k_{3 b}\right) u_{j}(t),
\end{aligned}
$$

das wiederum auf die $u$-Variable in Gl. (C.6) wirkt. Abb. C.2a zeigt das freilaufende System mit ausgedehnter Spiraldynamik, die nach Aktivierung der Regelung und Abwarten der Transientendynamik zu (von oben nach unten) laufenden Wellen umgewandelt wird (Abb. C.2c). Mit dieser Parameterkombination ist die gezeigte Dynamik zeitlich sehr stabil. Dass die Wellenfronten der ebenen Wellen nicht exakt gerade sind, liegt an den Anfangsbedingungen. So verläuft der Beginn der Wellenstabilisierung/-erzeugung nicht auf der gesamten Breite der Kontrollzellen einheitlich, so dass die Wellenfronten erst nach einer langen Transienten vollständig begradigt sind (bei fehlenden weiteren Inhomogenitäten).

Entsprechendes Vorgehen liefert für NFF bestehend aus drei parallel geschalteten Kerbfiltern (3.2), (3.3) anstelle von MDFC die in Abb. C.2d-f gezeigten Verhältnisse unter Einsatz der Regelparameter $k_{1}=2.1, k_{2}=2.35, k_{3}=2.05, R_{1}=140.4, R_{2}=788.7$, $R_{3}=2060.0, C=0.013, Q_{1}=11.5, Q_{2}=11.47, Q_{3}=11.97$. Wieder wird die freilaufende turbulente Dynamik aus Abb. C.2d von Transienten abgelöst, sobald die Regelung 
(a)

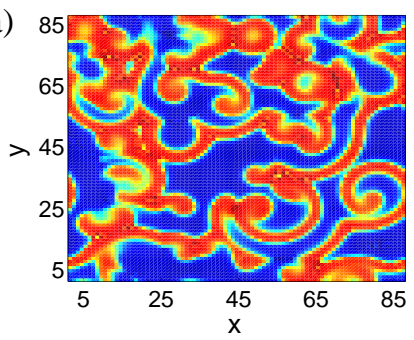

(d)

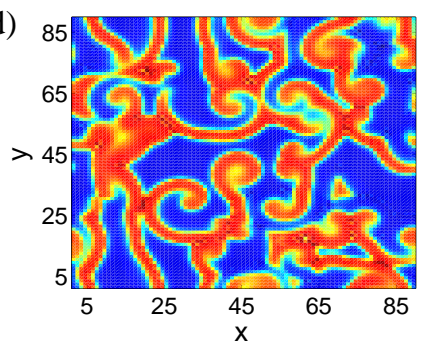

(b)

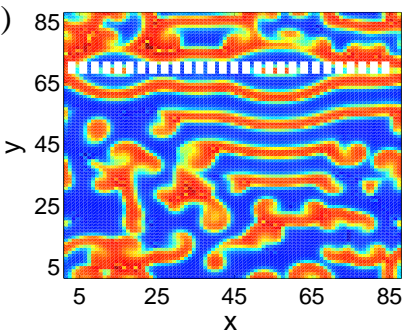

(e)

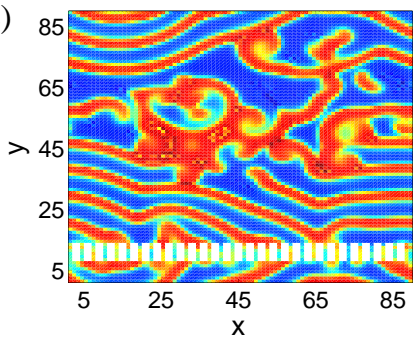

(c)

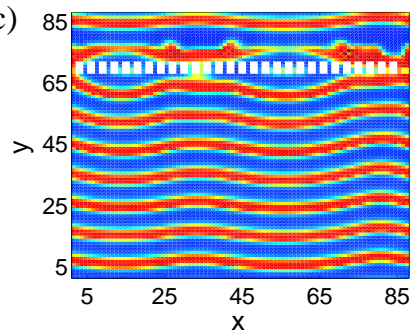

(f)

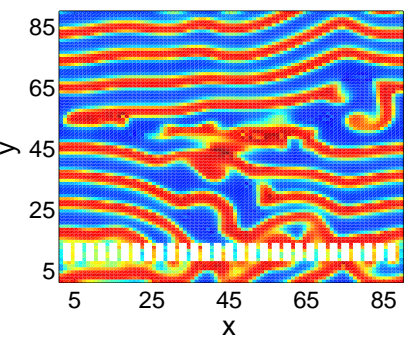

Abb. C.2: Farbkodierte Darstellung der raum-zeitlichen Entwicklung der FitzhughNagumo-Gleichung geregelt durch MDFC (a-c) und NFF (d-f). (a),(d) freilaufendes System, (b),(e) Transienten und (c),(f) Zieldynamik.

eingeschaltet wird. Die finale Dynamik kann Abb. C.2f entnommen werden, wobei keine geraden Wellenfronten im gesamten dynamischen Gebiet zu sehen sind. Das erklärt sich daraus, dass die Kontrollzellen laufende Wellen in beide Richtungen emittieren, die je nach Ausbreitungsgeschwindigkeit durch die periodischen Randbedingungen in einem anderen Bereich des Integrationsgebietes wieder aufeinander treffen. An diesen Stellen kann es, wie in Abb. C.2f zu sehen, zu erneuter irregulärer Dynamik kommen, was für MDFC aufgrund der höheren erwirkten Stabilität unkritischer als für NFF ist (Abb. C.2c). (Diese höhere Stabilität resultiert aus der besseren Anpassung von MDFC an das dynamische System.) Um auch für NFF stabilere Wellen zu ermöglichen, müssen zusätzliche Kontrollzellen aktiviert oder Kontrollzellen mit größerer Ausdehnung verwendet werden.

Insgesamt kann festgehalten werden, dass sowohl mit MDFC als auch mit NFF eine Umwandlung von Spiral- in laufende Wellen vorgenommen werden kann. Da das FitzhughNagumo-System (C.6) einen Prototypen für die menschliche Herzdynamik darstellt, ist MDFC und auch NFF vielleicht eine zukünftige schonende Alternative zur bisher angewendeten Defibrilation. 


\section{Literaturverzeichnis}

[1] H. D. I. Abarbanel, Z. Gills, C. Liu, and R. Roy. Nonlinear-time-series analysis of chaotic laser dynamics. Physical Review A (Atomic, Molecular, and Optical Physics), 53(1):440-453, 1996.

[2] H. D. I. Abarbanel, N. F. Rulkov, and M. M. Sushchik. Generalized synchronization of chaos: The auxiliary system approach. Physical Review E (Statistical Physics, Plasmas, Fluids, and Related Interdisciplinary Topics), 53(5):4528-4535, 1996.

[3] C. T. Abdallah, P. Dorato, J. Benitez Read, and R. Byrne. Delayed positive feedback can stabilize oscillatory system. In American Control Conference, volume 742, pages 3106-3107, 1993.

[4] A. Ahlborn and U. Parlitz. Chaos control of an intracavity frequency-doubled nd:yag laser. In Experimental chaos: 8th Experimental Chaos Conference, volume 742, pages 241-246. AIP, 2004.

[5] A. Ahlborn and U. Parlitz. Stabilizing unstable steady states using multiple delay feedback control. Physical Review Letters, 93(26):264101, 2004.

[6] A. Ahlborn and U. Parlitz. Controlling dynamical systems using multiple delay feedback control. Physical Review E (Statistical, Nonlinear, and Soft Matter Physics), 72(1):016206, 2005.

[7] A. Ahlborn and U. Parlitz. Multiple delay feedback control - a delay method for stabilizing fixed points. In Fifth EUROMECH Nonlinear Dynamics Conference ENOC-2005, August 7-12, 2005, Eindhoven (Netherlands), pages 1246-1254, 2005.

[8] A. Ahlborn and U. Parlitz. Chaos control using notch filter feedback. Physical Review Letters, 96(3):034102, 2006.

[9] A. Ahlborn and U. Parlitz. Laser stabilization with multiple-delay feedback control. Optics Letters, 31(4):465-467, 2006. 
[10] V. Ahlers, U. Parlitz, and W. Lauterborn. Hyperchaotic dynamics and synchronization of external-cavity semiconductor lasers. Physical Review E (Statistical Physics, Plasmas, Fluids, and Related Interdisciplinary Topics), 58(6):7208-7213, 1998.

[11] E. Allaria, F. T. Arecchi, A. D. Garbo, and R. Meucci. Synchronization of homoclinic chaos. Physical Review Letters, 86(5):791-794, 2001.

[12] S. Alonso, F. Sagues, and A. S. Mikhailov. Taming winfree turbulence of scroll waves in excitable media. Science, 299(5613):1722-1725, 2003.

[13] I. Aranson, H. Levine, and L. Tsimring. Controlling spatiotemporal chaos. Physical Review Letters, 72(16):2561-2564, 1994.

[14] J. Argyris, G. Faust, and M. Haase. Die Erforschung des Chaos. Vieweg Verlag, Braunschweig, 1994.

[15] N. Baba, A. Amann, E. Schöll, and W. Just. Giant improvement of time-delayed feedback control by spatio-temporal filtering. Physical Review Letters, 89(7):074101, 2002.

[16] T. Baer. Large-amplitude fluctuations due to longitudinal mode coupling in diodepumped intracavity-doubled nd:yag lasers. Journal of the Optical Society of America B: Optical Physics, 3(9):1175-1180, 1986.

[17] D. Barkley. A model for fast computer simulation of waves in excitable media. Physica, 49D:61-70, 1991.

[18] D. Barkley, M. Kness, and L. S. Tuckerman. Spiral-wave dynamics in a simple model of excitable media: The transition from simple to compound rotation. Physical Review A (Atomic, Molecular, and Optical Physics), 42(4):2489-2492, 1990.

[19] D. Battogtokh and A. Mikhailov. Controlling turbulence in the complex ginzburglandau equation. Physica D, 90:84-95, 1995.

[20] O. Beck, A. Amann, E. Schöll, J. E. S. Socolar, and W. Just. Comparison of timedelayed feedback schemes for spatiotemporal control of chaos in a reaction-diffusion system with global coupling. Physical Review E (Statistical, Nonlinear, and Soft Matter Physics), 66(1):016213, 2002.

[21] M. Bertram, C. Beta, M. Pollmann, A. S. Mikhailov, H. H. Rotermund, and G. Ertl. Pattern formation on the edge of chaos: Experiments with co oxidation on a pt(110) surface under global delayed feedback. Physical Review E (Statistical, Nonlinear, and Soft Matter Physics), 67(3):036208, 2003.

[22] M. Bertram and A. S. Mikhailov. Pattern formation in a surface chemical reaction with global delayed feedback. Physical Review E (Statistical, Nonlinear, and Soft Matter Physics), 63(6):066102, 2001. 
[23] M. Bertram and A. S. Mikhailov. Pattern formation on the edge of chaos: Mathematical modeling of co oxidation on a pt(110) surface under global delayed feedback. Physical Review E (Statistical, Nonlinear, and Soft Matter Physics), 67(3):036207, 2003.

[24] C. Beta and A. S. Mikhailov. Controlling spatio-temporal chaos in oscillatory reaction-diffusion systems by time-delay autosynchronization. Physica D, 199:173$184,2004$.

[25] I. V. Biktasheva. Drift of spiral waves in the complex ginzburg-landau equation due to media inhomogeneities. Physical Review E (Statistical Physics, Plasmas, Fluids, and Related Interdisciplinary Topics), 62(6):8800-8803, 2000.

[26] J. N. Blakely, L. Illing, and D. J. Gauthier. Controlling fast chaos in delay dynamical systems. Physical Review Letters, 92(19):193901, 2004.

[27] M. E. Bleich, D. Hochheiser, J. V. Moloney, and J. E. S. Socolar. Controlling extended systems with spatially filtered, time-delayed feedback. Physical Review E (Statistical Physics, Plasmas, Fluids, and Related Interdisciplinary Topics), 55(3):21192126, 1997.

[28] M. E. Bleich and J. E. S. Socolar. Controlling spatiotemporal dynamics with timedelay feedback. Physical Review E (Statistical Physics, Plasmas, Fluids, and Related Interdisciplinary Topics), 54(1):R17-R20, 1996.

[29] S. Boccaletti, E. Allaria, R. Meucci, and F. T. Arecchi. Experimental characterization of the transition to phase synchronization of chaotic co[sub 2] laser systems. Physical Review Letters, 89(19):194101, 2002.

[30] C. Bracikowski and R. Roy. Chaos in a multimode solid-state laser system. Chaos: An Interdisciplinary Journal of Nonlinear Science, 1(1):49-64, 1991.

[31] I. Bronstein, K. Semendjajew, and G. Musiol. Taschenbuch der Mathematik. Verlag Harri Deutsch, Frankfurt/Main, 1999.

[32] S. A. Campbell, I. Ncube, and J. Wu. Multistability and stable asynchronous periodic oscillations in a multiple-delayed neural system. Physica D, 214:101-119, 2006.

[33] T. W. Carr and I. B. Schwartz. Controlling the unstable steady state in a multimode laser. Physical Review E (Statistical Physics, Plasmas, Fluids, and Related Interdisciplinary Topics), 51(5):5109-5111, 1995.

[34] A. Chang, J. C. Bienfang, G. M. Hall, J. R. Gardner, and D. J. Gauthier. Stabilizing unstable steady states using extended time-delay autosynchronization. Chaos: An Interdisciplinary Journal of Nonlinear Science, 8(4):782-790, 1998. 
[35] G. Chen. Controlling Chaos and Bifurcations in Engineering Systems. CRC Press, Boca Raton, 2000.

[36] P. Colet, R. Roy, and K. Wiesenfeld. Controlling hyperchaos in a multimode laser model. Physical Review E (Statistical Physics, Plasmas, Fluids, and Related Interdisciplinary Topics), 50(5):3453-3457, 1994.

[37] M. C. Cross and P. C. Hohenberg. Pattern formation outside of equilibrium. Reviews of Modern Physics, 65(3):851-1112, 1993.

[38] R. V. Culshaw, S. Ruan, and G. Webb. A mathematical model of cell-to-cell spread of hiv-1 that includes a time delay. Journal of Mathematical Biology, 46:425-444, 2003.

[39] K. M. Cuomo and A. V. Oppenheim. Circuit implementation of synchronized chaos with applications to communications. Physical Review Letters, 71(1):65-68, 1993.

[40] D. J. DeShazer, R. Breban, E. Ott, and R. Roy. Detecting phase synchronization in a chaotic laser array. Physical Review Letters, 87(4):044101, 2001.

[41] U. Dressler, T. Ritz, A. S. zu Schweinsberg, R. Doerner, B. Hübinger, and W. Martienssen. Tracking unstable periodic orbits in a bronze ribbon experiment. Physical Review E, 51(3):1845-1848, Mar 1995.

[42] V. M. Eguíluz, E. Hernández-García, and O. Piro. Complex ginzburg-landau equation in the presence of walls and corners. Physical Review E (Statistical, Nonlinear, and Soft Matter Physics), 64(3):036205, 2001.

[43] J. Epaminondas Rosa, E. Ott, and M. H. Hess. Transition to phase synchronization of chaos. Physical Review Letters, 80(8):1642-1645, 1998.

[44] J. D. Farmer. Chaotic attractors of an infinite-dimensional dynamical system. Physica $4 D$, pages 366-393, 1982.

[45] F. H. Fenton, E. M. Cherry, H. M. Hastings, and S. J. Evans. Multiple mechanisms of spiral wave breakup in a model of cardiac electrical activity. Chaos: An Interdisciplinary Journal of Nonlinear Science, 12(3):852-892, 2002.

[46] H. foo Chen and J. ming Liu. Complete phase and amplitude synchronization of broadband chaotic optical fields generated by semiconductor lasers subject to optical injection. Physical Review E (Statistical, Nonlinear, and Soft Matter Physics), 71(4):046216, 2005.

[47] G. Franceschini, S. Bose, and E. Schöll. Control of chaotic spatiotemporal spiking by time-delay autosynchronization. Physical Review E (Statistical Physics, Plasmas, Fluids, and Related Interdisciplinary Topics), 60(5):5426-5434, 1999. 
[48] T. Frisch, S. Rica, P. Coullet, and J. M. Gilli. Spiral waves in liquid crystal. Physical Review Letters, 72(10):1471-1474, 1994.

[49] D. J. Gauthier. Controlling lasers by use of extended time-delay autosynchronization. Optics Letters, 23(9):703-705, 1998.

[50] Z. Gills, C. Iwata, R. Roy, I. B. Schwartz, and I. Triandaf. Tracking unstable steady states: Extending the stability regime of a multimode laser system. Physical Review Letters, 69(22):3169-3172, 1992.

[51] D. M. Goldschmidt, V. S. Zykov, and S. C. Müller. Transition to irregular dynamics of spiral waves under two-channel feedback. Physical Review Letters, 80(23):52205223, 1998.

[52] S. A. Gourley and S. Ruan. Spatio-temporal delays in a nutrient -plankton model on a finite domain: linear stability and bifurcations. Applied Mathematics and Computations, 145:391-412, 2003.

[53] S. Grill, V. S. Zykov, and S. C. Müller. Feedback-controlled dynamics of meandering spiral waves. Physical Review Letters, 75(18):3368-3371, 1995.

[54] J. K. Hale. Theory of Functional Differential Equations. Appl. Math. Sci. 3, Springer Verlag, Berlin, 1977.

[55] I. Harrington and J. E. S. Socolar. Limitation on stabilizing plane waves via timedelay feedback. Physical Review E (Statistical, Nonlinear, and Soft Matter Physics), 64(5):056206, 2001.

[56] M. Hendrey, K. Nam, P. Guzdar, and E. Ott. Target waves in the complex ginzburglandau equation. Physical Review E (Statistical Physics, Plasmas, Fluids, and Related Interdisciplinary Topics), 62(6):7627-7631, 2000.

[57] J. L. Hindmarsh and R. M. Rose. A model of neuronal bursting using three coupled first order differential equations. In Proceedings of the Royal Society of London. Series B, Biological Sciences, volume 221, pages 87-102, 1984.

[58] P. Hövel and E. Schöll. Control of unstable steady states by time-delayed feedback methods. Physical Review E (Statistical, Nonlinear, and Soft Matter Physics), 72(4):046203, 2005.

[59] P. Hövel and J. E. S. Socolar. Stability domains for time-delay feedback control with latency. Physical Review E (Statistical, Nonlinear, and Soft Matter Physics), 68(3):036206, 2003.

[60] G. Hu, J. Xiao, L. O. Chua, and L. Pivka. Controlling spiral waves in a model of two-dimensional arrays of chua's circuits. Physical Review Letters, 80(9):1884-1887, 1998. 
[61] G. Hu, J. Xiao, J. Yang, F. Xie, and Z. Qu. Synchronization of spatiotemporal chaos and its applications. Physical Review E (Statistical Physics, Plasmas, Fluids, and Related Interdisciplinary Topics), 56(3):2738-2746, 1997.

[62] A. Hübler and E. Lüscher. Resonant stimulation and control of nonlinear oscillators. Naturwissenschaften, 76:67-69, 1995.

[63] E. R. Hunt. Stabilizing high-period orbits in a chaotic system: The diode resonator. Physical Review Letters, 67(15):1953-1955, 1991.

[64] K. Ikeda. Multiple-valued stationary state and its instability of the transmitted light by a ring cavity system. Optics Communications, 30:257-261, 1979.

[65] K. Ikeda, H. Daido, and O. Alimoto. Optical turbulence: chaotic behaviour of transmitted light from a ring cavity. Physical Review Letters, 45(9):709-712, 1980.

[66] S. Jakubith, H. H. Rotermund, W. Engel, A. von Oertzen, and G. Ertl. Spatiotemporal concentration patterns in a surface reaction: Propagating and standing waves, rotating spirals, and turbulence. Physical Review Letters, 65(24):3013-3016, 1990.

[67] G. E. James, E. M. Harrell II, C. Bracikowski, K. Wiesenfeld, and R. Roy. Elimination of chaos in an intracavity-doubled nd:yag laser. Optics Letters, 15(20):1141-1143, 1990.

[68] G. E. James, E. M. Harrell II, and R. Roy. Intermittency and chaos in intracavity doubled lasers. ii. Physical Review A (Atomic, Molecular, and Optical Physics), 41(5):2778-2790, 1990.

[69] L. Junge and U. Parlitz. Synchronization and control of coupled ginzburg-landau equations using local coupling. Physical Review E (Statistical Physics, Plasmas, Fluids, and Related Interdisciplinary Topics), 61(4):3736-3742, 2000.

[70] W. Just, H. Benner, and E. Reibold. Theoretical and experimental aspects of chaos control by time-delayed feedback. Chaos: An Interdisciplinary Journal of Nonlinear Science, 13(1):259-266, 2003.

[71] W. Just, T. Bernard, M. Ostheimer, E. Reibold, and H. Benner. Mechanism of time-delayed feedback control. Physical Review Letters, 78(2):203-206, 1997.

[72] W. Just, S. Popovich, A. Amann, N. Baba, and E. Schöll. Improvement of timedelayed feedback control by periodic modulation: Analytical theory of floquet mode control scheme. Physical Review E (Statistical, Nonlinear, and Soft Matter Physics), 67(2):026222, 2003.

[73] W. Just, E. Reibold, H. Benner, K. Kacperski, P. Fronczak, and J. Holyst. Limits of time-delayed feedback control. Physics Letters A, 254:158-164, 1999. 
[74] K. Kaneko. Pattern dynamics in spatiotemporal chaos. Physica D, 34:1-41, 1989.

[75] K. Kaneko. Spatiotemporal chaos in one- and two-dimensional coupled map lattices. Physica D, 37:60-82, 1989.

[76] D. I. Kim, D. Lee, Y. Park, G. U. Kim, and C. Kim. Phase synchronizationof chaotic lasers. Optics Express, 14(2):702-709, 2006.

[77] M. Kim, M. Bertram, M. Pollmann, A. von Oertzen, A. S. Mikhailov, H. H. Rotermund, and G. Ertl. Spatiotemporal concentration patterns in a surface reaction: Propagating and standing waves, rotating spirals, and turbulence. Science, 92(5520):1357-1360, 2001.

[78] L. Kocarev and U. Parlitz. Generalized synchronization, predictability, and equivalence of unidirectionally coupled dynamical systems. Physical Review Letters, 76(11):1816-1819, 1996.

[79] K. Konishi, M. Hirai, and H. Kokame. Decentralized delayed-feedback control of a coupled map model for open flow. Physical Review E (Statistical Physics, Plasmas, Fluids, and Related Interdisciplinary Topics), 58(3):3055-3059, 1998.

[80] K. Konishi and H. Kokame. Observer-based delayed-feedback control for discretetime chaotic systems. Physics Letters A, 248:359-368, 1998.

[81] K. Konishi and H. Kokame. Decentralized delayed-feedback control of a one-way coupled ring map lattice. Physica D, 127:1-12, 1999.

[82] W. Lauterborn and T. Kurz. Coherent Optics. Springer Verlag, Berlin, 2003.

[83] J. Lee, J. Kim, G. H. Yi, and K. J. Lee. Unusual spiral wave tip trajectories in a parametrically forced nonequilibrium system. Physical Review E (Statistical, Nonlinear, and Soft Matter Physics), 65(4):046207, 2002.

[84] C. Liu, R. Roy, H. D. I. Abarbanel, Z. Gills, and K. Nunes. Influence of noise on chaotic laser dynamics. Physical Review E (Statistical Physics, Plasmas, Fluids, and Related Interdisciplinary Topics), 55(6):6483-6500, 1997.

[85] H. Ludyk. Theoretische Regelungstechnik. Springer Verlag, Berlin, 1995.

[86] J. Lunze. Regelungstechnik 1 (Systemtheoretische Grundlagen, Analyse und Entwurf einschleifiger Regelungen). Springer Verlag, 2006.

[87] M. C. Mackey and L. Glass. Oscillations and chaos in physiological control systems. Science, 197:287, 1977. 
[88] S. Madruga, S. Boccaletti, and M. A. Matías. Effect of a variable delay in delayed dynamical systems. International Journal of Bifurcation and Chaos, 11(11):2875$2280,2001$.

[89] R.-M. Mantel and D. Barkley. Periodic forcing of spiral waves in excitable media. Physical Review E (Statistical Physics, Plasmas, Fluids, and Related Interdisciplinary Topics), 54(5):4791-4802, 1996.

[90] C. M. Marcus and R. M. Westervelt. Stability of analog neural networks with delay. Physical Review A (General Physics), 39(1):347-359, 1989.

[91] A. C. Marti, M. Ponce, and C. Masoller. Steady-state stabilization due to random delays in maps with self-feedback loops and in globally delayed-coupled maps. Physical Review E (Statistical, Nonlinear, and Soft Matter Physics), 72(6):066217, 2005.

[92] C. Masoller and A. C. Marti. Random delays and the synchronization of chaotic maps. Physical Review Letters, 94(13):134102, 2005.

[93] D. Maza, A. Vallone, H. Mancini, and S. Boccaletti. Experimental phase synchronization of a chaotic convective flow. Physical Review Letters, 85(26):5567-5570, 2000 .

[94] R. McAllister, R. Meucci, D. DeShazer, and R. Roy. Competition between two frequencies for phase synchronization of a chaotic laser. Physical Review E (Statistical, Nonlinear, and Soft Matter Physics), 67(1):015202, 2003.

[95] K. B. Migler and R. B. Meyer. Spirals in liquid crystals in a rotating magnetic field. Physica D, 71(4):412-420, 1994.

[96] A. S. Mikhailov and K. Showalter. Control of waves, patterns and turbulence in chemical systems. Physics Reports, 425:79-194, 2006.

[97] M. Münkel, F. Kaiser, and O. Hess. Stabilization of spatiotemporally chaotic semiconductor laser arrays by means of delayed optical feedback. Physical Review E (Statistical Physics, Plasmas, Fluids, and Related Interdisciplinary Topics), 56(4):38683875, 1997.

[98] K. Myneni, T. A. Barr, N. J. Corron, and S. D. Pethel. New method for the control of fast chaotic oscillations. Physical Review Letters, 83(11):2175-2178, 1999.

[99] H. Nakajima. On analytical properties of delayed feedback control of chaos. Physics Letters A, 232:207-210, 1997.

[100] H. Nakajima. A rigorous proof for impossibility of a direct application of unstable delayed feedback control for time-continuous systems. Physics Letters A, 310(4):265268, 2003. 
[101] H. Nakajima and Y. Ueda. Limitation of generalized delayed feedback control. Physica D, 111:143-150, 1998.

[102] A. Namajunas, K. Pyragas, and A. Tamasevicius. Stabilization of an unstable steady state in a mackey-glass system. Physics Letters A, 204:255-262, 1995.

[103] S. Nasuno, N. Yoshimo, and S. Kai. Structural transition and motion of domain walls in liquid crystals under a rotating magnetic field. Physical Review E (Statistical Physics, Plasmas, Fluids, and Related Interdisciplinary Topics), 51(2):1598-1601, 1995.

[104] M. Oka and S. Kubota. Stable intracavity doubling of orthogonal linearly polarized modes in diode-pumped nd:yag lasers. Optics Letters, 13(10):805-807, 1988.

[105] G. V. Osipov, B. V. Shulgin, and J. J. Collins. Controlled movement and suppression of spiral waves in excitable media. Physical Review E (Statistical Physics, Plasmas, Fluids, and Related Interdisciplinary Topics), 58(6):6955-6958, 1998.

[106] E. Ott, C. Grebogi, and J. A. Yorke. Controlling chaos. Physical Review Letters, 64(11):1196-1199, 1990.

[107] A. V. Panfilov, S. C. Müller, V. S. Zykov, and J. P. Keener. Elimination of spiral waves in cardiac tissue by multiple electrical shocks. Physical Review E (Statistical Physics, Plasmas, Fluids, and Related Interdisciplinary Topics), 61(4):4644-4647, 2000.

[108] U. Parlitz, L. Junge, W. Lauterborn, and L. Kocarev. Experimental observation of phase synchronization. Physical Review E (Statistical Physics, Plasmas, Fluids, and Related Interdisciplinary Topics), 54(2):2115-2117, 1996.

[109] P. Parmananda. Tracking fixed-point dynamics in an electrochemical system using delayed-feedback control. Physical Review E (Statistical, Nonlinear, and Soft Matter Physics), 67(4):045202, 2003.

[110] P. Parmananda, M. Hildebrand, and M. Eiswirth. Controlling turbulence in coupled map lattice systems using feedback techniques. Physical Review E (Statistical Physics, Plasmas, Fluids, and Related Interdisciplinary Topics), 56(1):239-244, 1997.

[111] P. Parmananda and J. L. Hudson. Controlling spatiotemporal chemical chaos using delayed feedback. Physical Review E (Statistical, Nonlinear, and Soft Matter Physics), 64(3):037201, 2001.

[112] P. Parmananda, R. Madrigal, M. Rivera, L. Nyikos, I. Z. Kiss, and V. Gaspar. Stabilization of unstable steady states and periodic orbits in an electrochemical system using delayed-feedback control. Physical Review E (Statistical Physics, Plasmas, Fluids, and Related Interdisciplinary Topics), 59(5):5266-5271, 1999. 
[113] L. M. Pecora and T. L. Carroll. Synchronization in chaotic systems. Physical Review Letters, 64(8):821-824, 1990.

[114] V. Petrov, Q. Ouyang, and H. L. Swinney. Resonant pattern formation in a chemical system. Nature, 388:655-667, 1997.

[115] A. Pikovsky. Phase synchronization of chaotic oscillations by a periodic external field. Sov. J. Commun. Technol. Electron, 30:85-90, 1985.

[116] A. D. Poularikas. The Handbook of Formulas and Tables for Signal Processing. CRC Press LLC, Boca Raton, 1999.

[117] K. Pyragas. Continuous control of chaos by self-controlling feedback. Physics Letters A, 170(6):421-428, 1992.

[118] K. Pyragas. Control of chaos via an unstable delayed feedback controller. Physical Review Letters, 86(11):2265-2268, 2001.

[119] K. Pyragas, F. Lange, T. Letz, J. Parisi, and A. Kittel. Stabilization of an unstable steady state in intracavity frequency-doubled lasers. Physical Review E (Statistical Physics, Plasmas, Fluids, and Related Interdisciplinary Topics), 61(4):3721-3731, 2000.

[120] K. Pyragas, F. Lange, T. Letz, J. Parisi, and A. Kittel. Stabilization of an unstable steady state in intracavity frequency-doubled lasers. Physical Review E (Statistical Physics, Plasmas, Fluids, and Related Interdisciplinary Topics), 61(4):3721-3731, 2000 .

[121] K. Pyragas, F. Lange, T. Letz, J. Parisi, and A. Kittel. Dynamics and control of a multimode laser: Reduction of space-dependent rate equations to a lowdimensional system. Physical Review E (Statistical, Nonlinear, and Soft Matter Physics), 63(1):016204, 2001.

[122] K. Pyragas, V. Pyragas, I. Z. Kiss, and J. L. Hudson. Stabilizing and tracking unknown steady states of dynamical systems. Physical Review Letters, 89(24):244103, 2002.

[123] K. Pyragas, V. Pyragas, I. Z. Kiss, and J. L. Hudson. Adaptive control of unknown unstable steady states of dynamical systems. Physical Review E (Statistical, Nonlinear, and Soft Matter Physics), 70(2):026215, 2004.

[124] V. Pyragas and K. Pyragas. Delayed feedback control of the lorenz system: An analytical treatment at a subcritical hopf bifurcation. Physical Review E (Statistical, Nonlinear, and Soft Matter Physics), 73(3):036215, 2006. 
[125] W. J. Rappel, F. Fenton, and A. Karma. Spatiotemporal control of wave instabilities in cardiac tissue. Physical Review Letters, 83(2):456-459, 1999.

[126] W. P. Risk, T. R. Gosnell, and A. V. Nurmikko. Compact Blue-Green Lasers. Cambridge University Press, New York, 2000.

[127] M. C. Romano, M. Thiel, J. Kurths, I. Z. Kiss, and J. L. Hudson. Detection of synchronization for non-phase-coherent and non-stationary data. Europhysics Letters, 71(3):466-472, 2005.

[128] M. G. Rosenblum, L. Cimponeriu, A. Bezerianos, A. Patzak, and R. Mrowka. Identification of coupling direction: Application to cardiorespiratory interaction. Physical Review E (Statistical, Nonlinear, and Soft Matter Physics), 65(4):041909, 2002.

[129] M. G. Rosenblum, A. S. Pikovsky, and J. Kurths. Phase synchronization of chaotic oscillators. Physical Review Letters, 76(11):1804-1807, 1996.

[130] M. G. Rosenblum, A. S. Pikovsky, and J. Kurths. From phase to lag synchronization in coupled chaotic oscillators. Physical Review Letters, 78(22):4193-4196, 1997.

[131] R. Roy, T. W. Murphy, Jr., T. D. Maier, Z. Gills, and E. R. Hunt. Dynamical control of a chaotic laser: Experimental stabilization of a globally coupled system. Physical Review Letters, 68(9):1259-1262, 1992.

[132] S. Ruan. Delay Differential Equations in Single Species Dynamics, volume 205. Springer, Berlin, 2005.

[133] O. Rudzick and A. S. Mikhailov. Front reversals, wave traps, and twisted spirals in periodically forced oscillatory media. Physical Review Letters, 96(1):018302, 2006.

[134] N. F. Rulkov, M. M. Sushchik, L. S. Tsimring, and H. D. I. Abarbanel. Generalized synchronization of chaos in directionally coupled chaotic systems. Physical Review E (Statistical Physics, Plasmas, Fluids, and Related Interdisciplinary Topics), 51(2):980-994, 1995.

[135] L. A. Safonov, E. Tomer, V. V. Strygin, Y. Ashkenazy, and S. Havlin. Multifractional chaotic attractors in a system of delay-differential equations modelling road trafffic. Chaos, 12(4):1006-1014, 2002.

[136] H. G. Schuster. Handbook of Chaos Control. Wiley-VCH, Weinheim, 1999.

[137] H. G. Schuster and M. B. Stemmler. Control of chaos by oscillating feedback. Physical Review E (Statistical Physics, Plasmas, Fluids, and Related Interdisciplinary Topics), 56(6):6410-6417, 1997.

[138] A. S. Schweinsberg. Stabilisierung und Charakterisierung eines frequenzverdoppelten Nd:YAG-Lasers. Dissertation, Der andere Verlag, Berlin, 2000. 
[139] S. Sivaprakasam, I. Pierce, P. Rees, P. S. Spencer, K. A. Shore, and A. Valle. Inverse synchronization in semiconductor laser diodes. Physical Review A (Atomic, Molecular, and Optical Physics), 64(1):013805, 2001.

[140] J. E. S. Socolar and D. J. Gauthier. Analysis and comparison of multiple-delay schemes for controlling unstable fixed points of discrete maps. Physical Review E (Statistical Physics, Plasmas, Fluids, and Related Interdisciplinary Topics), 57(6):65896595, 1998.

[141] J. E. S. Socolar, D. W. Sukow, and D. J. Gauthier. Stabilizing unstable periodic orbits in fast dynamical systems. Physical Review E (Statistical Physics, Plasmas, Fluids, and Related Interdisciplinary Topics), 50(4):3245-3248, 1994.

[142] O. V. Sosnovtseva, A. G. Balanov, T. E. Vadivasova, V. V. Astakhov, and E. Mosekilde. Loss of lag synchronization in coupled chaotic systems. Physical Review E (Statistical Physics, Plasmas, Fluids, and Related Interdisciplinary Topics), 60(6):6560-6565, 1999.

[143] J. C. Sprott. Some simple chaotic flows. Physical Review E (Statistical Physics, Plasmas, Fluids, and Related Interdisciplinary Topics), 50(2):R647-R650, 1994.

[144] R. Stang. Regelungstechnik. Klöckner and Möller, 1992.

[145] D. W. Sukow, M. E. Bleich, D. J. Gauthier, and J. E. S. Socolar. Controlling chaos in a fast diode resonator using extended time-delay autosynchronization: Experimental observations and theoretical analysis. Chaos: An Interdisciplinary Journal of Nonlinear Science, 7(4):560-576, 1997.

[146] P. Tass, M. G. Rosenblum, J. Weule, J. Kurths, A. Pikovsky, J. Volkmann, A. Schnitzler, and H.-J. Freund. Detection of n:m phase locking from noisy data: Application to magnetoencephalography. Physical Review Letters, 81(15):3291-3294, 1998.

[147] C. M. Ticos, J. Epaminondas Rosa, W. B. Pardo, J. A. Walkenstein, and M. Monti. Experimental real-time phase synchronization of a paced chaotic plasma discharge. Physical Review Letters, 85(14):2929-2932, 2000.

[148] U. Tietze and C. Schenk. Electronic Circuits : Handbook for Design and Application. Springer Verlag, Berlin, 2004.

[149] C. K. Tung and C. K. Chan. Dynamics of spiral waves under phase feedback control in a belousov-zhabotinsky reaction. Physical Review Letters, 89(24):248302, 2002.

[150] H. Unbehauen. Regelungstechnik 1. Vieweg Verlag, 2007. 
[151] V. I. Ustyugov, O. A. Orlov, M. M. Khaleev, G. E. Novikov, E. A. Viktorov, and P. Mandel. Self-stabilization of a continuous wave multimode green laser due to antiphase dynamics. Applied Physics Letters, 71(2):154-156, 1997.

[152] H. Varela, C. Beta, A. Bonnefont, and K. Krischer. Transitions to electrochemical turbulence. Physical Review Letters, 94(17):174104, 2005.

[153] I. Wedekind and U. Parlitz. Synchronization and antisynchronization of chaotic power drop-outs and jump-ups of coupled semiconductor lasers. Physical Review E (Statistical, Nonlinear, and Soft Matter Physics), 66(2):026218, 2002.

[154] J. Wei and S. Ruan. Stability and bifurcation in a neural network model with two delays. Physica D, 130:255-272, 1999.

[155] K. Wiesenfeld, C. Bracikowski, G. James, and R. Roy. Observation of antiphase states in a multimode laser. Physical Review Letters, 65(14):1749-1752, 1990.

[156] D. Xiao and S. Ruan. Multiple bifurcations in a delayed predator-prey system with nonmonotonic functional response. J. Differential Equations, 176:494-510, 2001.

[157] A. S. zu Schweinsberg and U. Dressler. Characterization and stabilization of the unstable fixed points of a frequency doubled nd:yag laser. Physical Review E (Statistical, Nonlinear, and Soft Matter Physics), 63(5):056210, 2001.

[158] V. Zykov and H. Engel. Dynamics of spiral waves under global feedback in excitable domains of different shapes. Physical Review E (Statistical, Nonlinear, and Soft Matter Physics), 70(1):016201, 2004.

[159] V. S. Zykov, G. Bordiougov, H. Brandtstädter, I. Gerdes, and H. Engel. Periodic forcing and feedback control of nonlinear lumped oscillators and meandering spiral waves. Physical Review E (Statistical, Nonlinear, and Soft Matter Physics), 68(1):016214, 2003.

[160] V. S. Zykov, G. Bordiougov, H. Brandtstädter, I. Gerdes, and H. Engel. Global control of spiral wave dynamics in an excitable domain of circular and elliptical shape. Physical Review Letters, 92(1):018304, 2004.

[161] V. S. Zykov and H. Engel. Feedback-mediated control of spiral waves. Physica D, 199:243-263, 2004.

[162] V. S. Zykov, A. S. Mikhailov, and S. C. Müller. Controlling spiral waves in confined geometries by global feedback. Physical Review Letters, 78(17):3398-3401, 1997. 


\section{Danksagung}

Ich danke Herrn Prof. Dr. W. Lauterborn für die Aufnahme in das Dritte Physikalische Institut und die Ermöglichung der Finanzierung dieser Arbeit.

Weiterhin möchte ich mich bei Herrn Prof. Dr. U. Parlitz für die Betreuung und Ermöglichung sowie die große Unterstützung im Zuge dieser Arbeit bedanken.

Bei Herrn Prof. Dr. E. Bodenschatz möchte ich mich für die Übernahme des Amtes des Korreferenten dieser Arbeit bedanken.

Zudem danke ich Prof. Dr. W. Lauterborn, Prof. Dr. T. Geisel, Prof. Dr. Münzenberg, und PD. Dr. C. Ronning dafür, dass sie sich als Prüfer bereiterklärt haben.

Außerdem gilt mein Dank der elektrischen Werkstatt für den Bau eines D/A-Wandlers und die Bestellung so mancher Bauteile sowie der feinmechanischen Werkstatt für den Bau mehrerer mechanischer Teile für den experimentellen Laseraufbau.

Zu guter letzt geht ein Dankeschön an meine Familie für die Ermöglichung meines Studiums und die Unterstützung während der ganzen Zeit, sowie an alle meine Freunde und meine Freundin. 


\title{
Lebenslauf
}

\author{
Name: Ahlborn \\ Vorname: Alexander \\ Geburtstag: $\quad$ 06.06.1979 \\ Geburtsort: Göttingen \\ Staatsangehörigkeit: deutsch
}

Schulbildung

1985 - 1989 Grundschule Bovenden

1989 - $1991 \quad$ Orientierungsstufe Bovenden

1991 - $1998 \quad$ Otto-Hahn-Gymnasium, Göttingen, Abitur

Zivildienst

$8.1998-9.1999 \quad$ Sozialstation Bovenden

Berufsausbildung

$10.1999-1.2004$

Studium der Physik, Georg-August-Universität, Göttingen

7.2001

Diplomvorprüfung in Physik (4. Semester)

1.2004

Diplom in Physik (9. Semester)

$2.2004-5.2004$

Wissenschaftliche Hilfskraft, Drittes Physikalisches Institut, Universität Göttingen

6.2004 - $6.2007 \quad$ Wissenschaftlicher Mitarbeiter, Drittes Physikalisches Institut, Universität Göttingen, Promotion

Veröffentlichungen

A. Ahlborn and U. Parlitz, in „Handbook of Chaos Control“, Wiley-VCH (2007)

A. Ahlborn and U. Parlitz, Phys. Rev. Lett. 96(3), 034102 (2006)

A. Ahlborn and U. Parlitz, Phys. Rev. Lett. 93(26), 264101 (2004)

A. Ahlborn and U. Parlitz, Opt. Lett. 31(4), 465-467 (2006)

A. Ahlborn and U. Parlitz, Phys. Rev. E 72(1), 016206 (2005)

A. Ahlborn and U. Parlitz, 8th Experimental Chaos Conference, 742, 241-246 (2004)

A. Ahlborn and U. Parlitz, Proceedings of ENOC-2005, 1246-1254 (2005)

Gutachter für Phys. Lett. A und Nonlinear Dynamics 